\title{
Non-linear Thermal Modeling Using Neural Networks
}

\author{
by \\ Aditi Das \\ Ottawa-Carleton Institute for Electrical and Computer Engineering \\ Department of Electronics \\ Faculty of Engineering \\ Carleton University \\ Ottawa, Canada
}

March 2006

(C) Aditi Das, 2006 


$\begin{array}{ll}\begin{array}{l}\text { Library and } \\ \text { Archives Canada }\end{array} & \begin{array}{l}\text { Bibliothèque et } \\ \text { Archives Canada }\end{array} \\ \begin{array}{l}\text { Published Heritage } \\ \text { Branch }\end{array} & \begin{array}{l}\text { Direction du } \\ \text { Patrimoine de l'édition }\end{array} \\ \begin{array}{l}\text { 395 Wellington Street } \\ \text { Ottawa ON K1A ON4 }\end{array} & \begin{array}{l}\text { 395, rue Wellington } \\ \text { Ottawa ON K1A ON4 } \\ \text { Canada }\end{array}\end{array}$

Your file Votre référence ISBN: 978-0-494-16454-9 Our file Notre référence ISBN: 978-0-494-16454-9

NOTICE:

The author has granted a nonexclusive license allowing Library and Archives Canada to reproduce, publish, archive, preserve, conserve, communicate to the public by telecommunication or on the Internet, loan, distribute and sell theses worldwide, for commercial or noncommercial purposes, in microform, paper, electronic and/or any other formats.

The author retains copyright ownership and moral rights in this thesis. Neither the thesis nor substantial extracts from it may be printed or otherwise reproduced without the author's permission.
AVIS:

L'auteur a accordé une licence non exclusive permettant à la Bibliothèque et Archives Canada de reproduire, publier, archiver, sauvegarder, conserver, transmettre au public par télécommunication ou par l'Internet, prêter, distribuer et vendre des thèses partout dans le monde, à des fins commerciales ou autres, sur support microforme, papier, électronique et/ou autres formats.

L'auteur conserve la propriété du droit d'auteur et des droits moraux qui protège cette thèse. $\mathrm{Ni}$ la thèse ni des extraits substantiels de celle-ci ne doivent être imprimés ou autrement reproduits sans son autorisation.
In compliance with the Canadian

Privacy Act some supporting forms may have been removed from this thesis.

While these forms may be included in the document page count, their removal does not represent any loss of content from the thesis.
Conformément à la loi canadienne sur la protection de la vie privée, quelques formulaires secondaires ont été enlevés de cette thèse.

Bien que ces formulaires aient inclus dans la pagination, il n'y aura aucun contenu manquant.

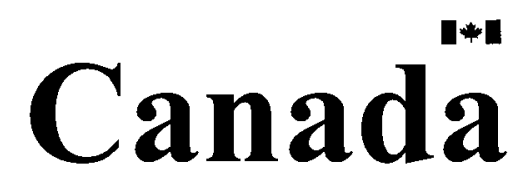




\begin{abstract}
In this thesis a neural network-based modeling technique is proposed in order to perform non-linear thermal analysis of package models. A detailed thermal model, which physically represents the actual device geometry, is broken into smaller sub-models that are linked to a base-model through thermal ports. Sub-models exchange heat-flows with the base-model. These sub-models, with well defined thermal ports, are able to produce all the temperatures of interest in the detailed model.
\end{abstract}

A non-linear neural model is developed by training the neural network with a set of training data obtained from Atar simulation of the sub-model and the final quality of the trained model is evaluated by testing it with a set of testing data. The number of inputs (i.e., the number of ports of the sub-model) for the neural network model is determined by performing a linear simulation on the sub-model for different boundary conditions. The importance of ports of the sub-model is evaluated by determining the errors at different temperatures of interest of the sub-model with port configuration with respect to the temperatures of the detailed model. It has been shown that the neural network is capable of building the non-linear thermal model of packages with sufficient accuracy.

Finally, a detailed thermal model is represented with a number of sub-models. Temperatures at different points of interest of the sub-model are determined in an 
iterative way by utilizing the Matlab/C code obtained from the neural network tool used in this thesis. It has been found that the model simulation becomes significantly faster when a detailed model is represented with a number of sub-models. 


\section{Acknowledgements}

I would like to take this opportunity to thank my supervisor Prof. Tom Smy for his expert guidance as well as continued financial and logistic supports without which this thesis wouldn't have been completed. My sincere thanks also go to Prof. Q. J. Zhang with who taught me the course on Neural Networks and has provided us with the necessary support on the Neuromodeler tool while carrying out this research.

I am grateful to my friends in Ottawa; specially, Mr. Rafat Alam, Mrs. Nusrat Aireen and their son Prethul for their enormous affection and support during my stay in Ottawa. I would also like to thank all the members of my research group. The discussions I have had with these people have been really rewarding for me.

Finally, I would like to express my profound appreciation to my family members for their love and support. 


\section{Contents}

Chapter 1: Introduction 1

$1.1 \quad$ Background $\ldots \ldots \ldots \ldots \ldots \ldots \ldots \ldots \ldots \ldots \ldots \ldots, 1$

$1.2 \quad$ Motivation $\ldots \ldots \ldots \ldots \ldots \ldots \ldots \ldots \ldots \ldots \ldots \ldots \ldots$

$1.3 \quad$ Thesis Methodology $\ldots \ldots \ldots \ldots \ldots \ldots \ldots \ldots \ldots \ldots \ldots \ldots$

$1.4 \quad$ Electro-Thermal Analogy $\ldots \ldots \ldots \ldots \ldots \ldots \ldots \ldots \ldots, \quad \ldots \ldots$

1.5 Organization of the Thesis ..................... 7

$\begin{array}{lll}\text { Chapter 2: } & \text { Thermal Issues in Packaging } & 8\end{array}$

2.1 Introduction to Packaging $\ldots \ldots \ldots \ldots \ldots \ldots \ldots \ldots \ldots$

$\begin{array}{llllllll}2.2 & \text { Thermal Packaging } \ldots \ldots \ldots \ldots & \ldots & \ldots & \ldots & \ldots & \ldots & \ldots\end{array}$

2.3 Thermal Equation for Conduction $\ldots \ldots \ldots \ldots \ldots \ldots \ldots$

$2.4 \quad$ Package Simulation and Modeling $\ldots \ldots \ldots \ldots \ldots \ldots \ldots$

2.4.1 Thermal Simulator - Atar ............... 17

2.4.2 Use of Sub-model in Thermal Modeling ....... 18

2.4.3 Thermal Modeling Tool-Neuromodeler ..... 22

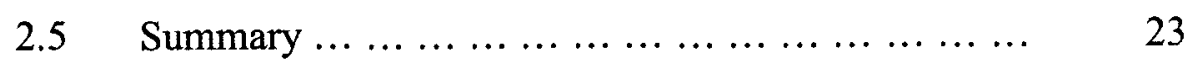


$\begin{array}{lll}\text { Chapter 3: } & \text { Neural Networks } & 24\end{array}$

3.1 Introduction to Neural Networks ................ 24

3.2 Neural Network Structures ................... 26

3.2.1 Multi Layer Perceptrons ... ... . . . . . . . ... 28

3.2.2 Radial Basis Function Networks . .......... 34

3.2.3 Wavelet Neural Networks ............... 34

3.2.4 Recurrent Neural Networks .............. $\quad 34$

3.3 Training of Neural Networks $\ldots \ldots \ldots \ldots \ldots \ldots \ldots$

3.3.1 Back Propagation .................... 38

3.3.2 Conjugate Gradient .................. 40

3.4 Quality Evaluation of Neural Networks ........... 42

$3.5 \quad$ Summary $\ldots \ldots \ldots \ldots \ldots \ldots \ldots \ldots \ldots \ldots \ldots \ldots$

Chapter 4: $\quad$ Sub-model Definition 45

4.1 Hierarchical Modeling Using Sub-model ......... 45

$4.2 \quad$ Sub-model Definition Using Atar ............. 49

4.3 Linear Simulation Results for Package Models ..... 51

4.3.1 GaN Based Power Package Amplifier ....... 51

4.3.2 GaAs Based Power Amplifier ... ......... 58

4.3.3 Package Model ...................... 64

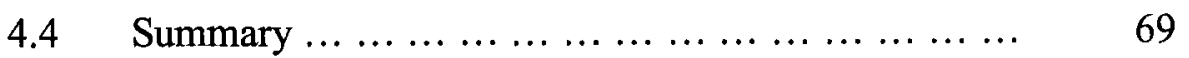


Chapter 5: Creation and Training of Neural Network

\section{Modeling}

$5.1 \quad$ Neural Network Modeling $\ldots \ldots \ldots \ldots \ldots \ldots \ldots \ldots \ldots$

5.2 GaN Based Power Package Amplifier ... ... . . . . . .. $\quad 72$

5.2.1 Training of GaN Model with Four-Port Configuration $\ldots \ldots \ldots \ldots \ldots \ldots \ldots \ldots \ldots \ldots$

5.2.2 Testing of GaN Model with Four-Port Configuration $\ldots \ldots \ldots \ldots \ldots \ldots \ldots \ldots \ldots$

5.2.3 Training of GaN Model with Five-Port Configuration

5.2.4 Testing of GaN Model with Five-Port Configuration $\ldots \ldots \ldots \ldots \ldots \ldots \ldots \ldots \ldots \quad 85$

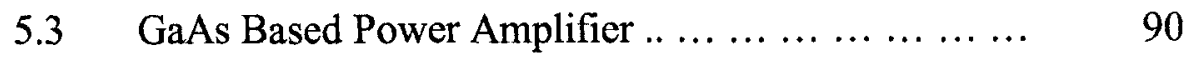

5.3.1 Training of GaAs Based Power Amplifier .... $\quad 90$

5.3.2 Testing of GaAs Based Power Amplifier ...... $\quad 92$

$\begin{array}{llllllllllll}5.4 & \text { Package Model } \ldots \ldots \ldots & \ldots & \ldots & \ldots & \ldots & \ldots & \ldots & \ldots & \ldots & \ldots & \ldots\end{array}$

5.4.1 Training of Package Model ................. $\quad 97$

5.4.2 Testing of Package Model . . . . . . . . . . . . . . . . $\quad 99$

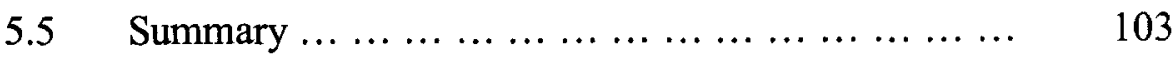


Chapter 6: Use of Neural Networks Models in Thermal $\begin{array}{ll}\text { Simulation } & 104\end{array}$

6.1 Use of Neural Networks Sub-models in Mixed-Model 104

6.2 Full Models Obtained from Neural Networks SubModels ..................................... 108

6.2.1 GaN Based Power Package Amplifier ........ 108

6.2.2 GaAs Based Power Amplifier ............ 116

6.2.3 Package Model ......................... 120

6.3 Speed-up Using Mixed Model . ... ... . . . . . . . . . . 127

$\begin{array}{llllllllllll}6.4 & \text { Summary } \ldots & \ldots & \ldots & \ldots & \ldots & \ldots & \ldots & \ldots & \ldots & \ldots & \ldots\end{array}$

$\begin{array}{lll}\text { Chapter 7: } & \text { Conclusions } & 129\end{array}$

$\begin{array}{llllllllllll}7.1 & \text { Summary } & \ldots & \ldots & \ldots & \ldots & \ldots & \ldots & \ldots & \ldots & \ldots & \ldots\end{array}$

7.2 Future Research Directions . ... . . . . . . . . . . . . . 131

$\begin{array}{lll}\text { Appendix A: } & \text { Simulation Results } & 133\end{array}$

Appendix B: Neural Network Modeling Results 154

Appendix C: Iteration Results 159 


\section{List of Figures}

2.1 A Schematic Illustration of Several Levels of Packaging Involved in an Electronic System $\ldots \ldots \ldots \ldots \ldots \ldots \ldots \ldots \ldots \ldots \ldots \ldots \ldots$

2.2 Schematic Representation of (a) A Mixed-Model Including a Twoport Sub-model Attached to a Base-Model (PCB) (b) Relationship between Components in a Mixed-model Using a Linear Sub-model ...

3.1 Multi Layer Perceptrons (MLP) Structure $\ldots \ldots \ldots \ldots \ldots \ldots \ldots \ldots$

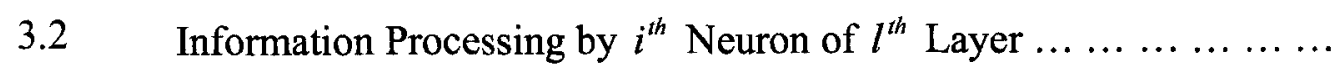

4.1 Atar Mixed-model Showing a Base-model, a Detailed Componentmodel and a Sub-model $\ldots \ldots \ldots \ldots \ldots \ldots \ldots \ldots \ldots \ldots \ldots \ldots$

4.2 GaN Based Power Amplifier Mesa Structure .................. 52

4.3 GaN Model (a) Component-model Attached to a Base-model (b) Submodel Attached to a Base-model $\ldots \ldots \ldots \ldots \ldots \ldots \ldots \ldots \ldots \ldots \ldots$

4.4 GaN Mixed-model Showing (a) Metal Ports (b) Metal Ports and Ports at the Bottom (c) Different Temperature Points (I1_1 - I3_6) of

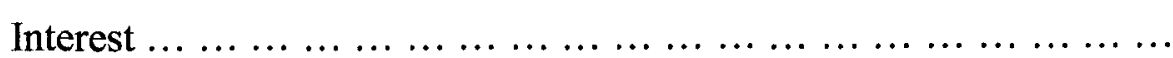

4.5 GaAs Power Amplifier (a) Component-model Showing Metal Ports and Ports at the Sides (b) Sub-model Attached to a Base-model ......

4.6 GaAs Sub-model Showing the Boundary Condition .............. 
4.7 Temperature Contour for the Sub-model of GaAs Model ......... 63

4.8 1/2 View of Package Model (a) Sub-model Attached to Base-model (b)

Component-model Attached to Base-model ................ 65

4.9 Top View of Package Model Showing (a) Three Ports (b) Different Points $(\mathrm{P} 1-\mathrm{P} 16)$ of Interest $\ldots \ldots \ldots \ldots \ldots \ldots \ldots \ldots \ldots \ldots \ldots \ldots \ldots$

5.1 Inputs and Outputs of the Developed Neural Network Model ... ..... 71

5.2 Training Error of GaN Model with Four-Port Configuration for Different Training Methods and Structures ...................

5.3 (a) Average and (b) Maximum Testing Error vs. Epoch for GaN-4 Model with Three-Layer Perceptrons Using Back Propagation

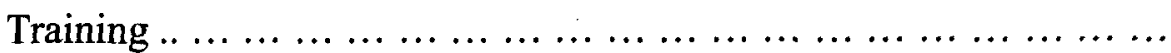

5.4 Percentage Error of Gan-4 Neural Model with Three-Layer Perceptrons for (a) T-linear Using Back Propagation Training (b) Tnonlinear Using Back Propagation Training . ...............

5.5 Neural Model Output vs. Test Data for GaN-4 Model Using ThreeLayer Perceptrons for (a) T-linear with Back Propagation Training (b) T-nonlinear with Conjugate Gradient Training ...............

5.6 (a) T-nonlinear and I1-1 (Outputs) vs. Power (Input) w.r.t. Bot (Input) for Three-Layer GaN-4 Model with Back Propagation Training (b) TM1 and TM3 (Outputs) vs. Power (Input) w.r.t. Bot (Input) for Three-Layer GaN-4 Model with Conjugate Gradient Training ........

5.7 Training Error of GaN Model with Five-Port Configuration for Different Training Methods and Structures $\ldots \ldots \ldots \ldots \ldots \ldots \ldots \ldots$ 
5.8 Percentage Error of Neural Model for TP1 Using Three-Layer GaN-5 Model with Back Propagation Training $\ldots \ldots \ldots \ldots \ldots \ldots \ldots \ldots$

5.9 (a) TP1 and TP5 (Outputs) vs. Port1 (Input) w.r.t Port5 (Input) for Three-Layer GaN-5 Model with Back Propagation Training (b) Tlinear and T-nonlinear (Outputs) vs. Port1 (Input) w.r.t Port5 (Input) for Three-Layer GaN-5 Model with Conjugate Gradient Training .. ...

5.10 Training Error of GaAs Model with Five-Port Configuration for Different Training Methods and Structures ...................

5.11 Percentage Error of Neural Model for GaAs with Three-Layer Perceptrons for (a) T-linear Using Conjugate Gradient Training (b) Tnonlinear Using Back Propagation Training . ................

5.12 Neural Model Output vs. Test Data for GaAs Model with Three-layer Perceptrons for (a) T-nonlinear Using Back Propagation Training (b) TP1 Using Conjugate Gradient Training ...................

5.13 (a) T-nonlinear and PM1 (Outputs) vs. Power (Input) w.r.t Port5 (Input) for Three-Layer GaAs Model with Back Propagation Training (b) TP2 and TP5 (Outputs) vs. Port2 (Input) w.r.t Port5 (Input) for Three-Layer GaAs Model with Conjugate Gradient Training .........

5.14 Training Error of Package Model with Three-Port Configuration for Different Training Methods and Structures ...................

5.15 Percentage Error of Neural Model for T-nonlinear Using Three-Layer Package Model with Conjugate Gradient Training .............

5.16 Neural Model Output vs. Test Data for T-nonlinear Using Three-

vii 
Layer Package Model with Back Propagation Training ............

5.17 (a) T-nonlinear and P16 (Outputs) vs. Alpha-top (Input) w.r.t T1 (Input) for Three-Layer Package Model with Back Propagation Training (b) T-alpha and TP1 (Outputs) vs. Alpha-top (Input) w.r.t T1 (Input) for Three-Layer Package Model with Back Propagation

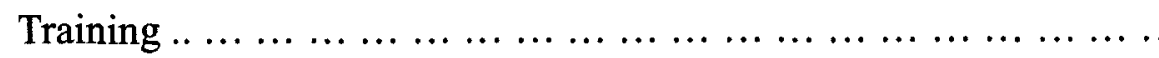
102

6.1 GaN Model (a) Sub-model Layout (b) Mixed-model Layout $\ldots \ldots \ldots . \quad 109$

$6.2 \quad$ Detailed GaN Model $\ldots \ldots \ldots \ldots \ldots \ldots \ldots \ldots \ldots \ldots \ldots \ldots \ldots$

6.3 (a) Details of GaN Sub-model Placement (b) GaN Full-model Showing GaN Amplifier Detail .

6.4 (a) Temperature of Detailed GaN Model (b) Details View of Temperature of Fully Detailed GaN Model (c) Temperature of the GaN Base-model Using Sub-model

6.5 (a) Temperature Error of GaN Sub-model with Four-Port Configuration (b) Detailed View of Temperature Error for Four-Port Configuration (c) Temperature Error of GaN Sub-model with Five-

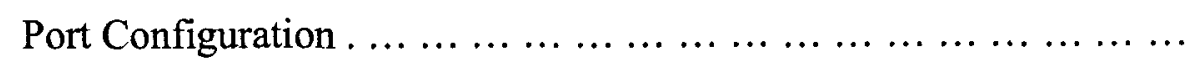
115

$6.6 \quad$ (a) GaAs Model (b) Detail GaAs Model .....................

6.7 (a) Top View of Temperature of GaAs Model (b) Detail Temperature of GaAs Model (c) Temperature Error in the Substrate of GaAs Model .

6.8 (a) Temperatures for Substrate of Package Model Using Detailed Model (b) Temperatures for Substrate of Package Model Using Sub- 


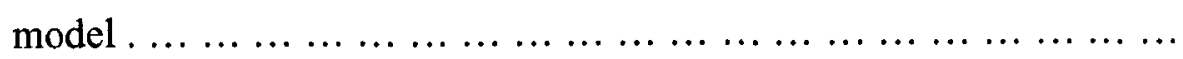

6.9 Temperature of Sub-models for Package Model $\ldots \ldots \ldots \ldots \ldots \ldots \ldots$

6.10 (a) Temperature Contours for Sub-models of Package Model (b) Temperature Contours for Sub-models of Package Model for Rightside BC (c) Temperatures of Package Model for Right-side BC ... ...

6.11 Temperature Errors of Sub-models for Package Model ............. 126 


\section{List of Tables}

1.1 An Analogy between Thermal and Electrical Quantities ........... 6

4.1 Different Scenarios for Linear Simulation of GaN Model .......... 53

4.2 The Output Temperatures (for Case-2 in Table 4.1) of GaN Model ... 55

4.3 Calculated Errors for Output Temperatures (for Case-2 in Table 4.1)

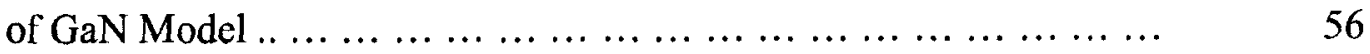

4.4 Average Errors for Output Temperatures of GaN Model ........... 57

4.5 Different Scenarios for Linear Simulation of GaAs Model .. ........ 58

4.6 The Output Temperatures (for Case-3 in Table 4.5) of GaAs Model .. $\quad 61$

4.7 Calculated Errors for Output Temperatures (for Case-3 in Table 4.5)

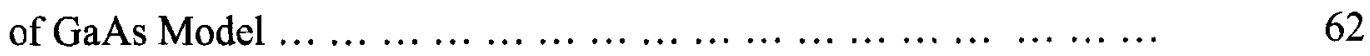

4.8 Average Errors for Output Temperatures of GaAs Model .......... 62

4.9 Different Scenarios for Linear Simulation of Package Model ... ...... 64

4.10 Output Temperatures and Corresponding Errors (for Case-5 in Table 4.9) of Package Model .............................. 67

4.11 Average Errors for Output Temperatures of Package Model ......... 68

5.1 Training Errors of GaN Model with Four-Port Configuration ... ...... 76

5.2 Training and Testing Results of GaN Model with Four-Port

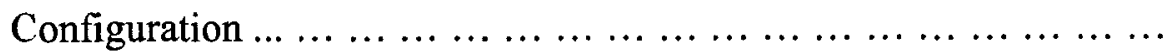


5.3 Testing Errors of GaN Model with Three-Layer Perceptrons and Four-Port Configuration $\ldots \ldots \ldots \ldots \ldots \ldots \ldots \ldots \ldots \ldots \ldots \ldots \ldots$

5.4 Training Errors of GaN Model with Five-Port Configuration ... ... ... $\quad 84$

5.5 Training and Testing Results of GaN Model with Five-Port

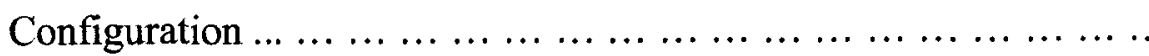

5.6 Testing Errors of GaN Model with Three-Layer Perceptrons and Five-Port Configuration $\ldots \ldots \ldots \ldots \ldots \ldots \ldots \ldots \ldots \ldots$

$5.7 \quad$ Training Errors of GaAs Model $\ldots \ldots \ldots \ldots \ldots \ldots \ldots \ldots \ldots \ldots \ldots$

$5.8 \quad$ Training and Testing Results for GaAs Model ................ 92

5.9 Testing Errors of GaAs Model with Three-Layer Perceptrons ... ... ... 93

$5.10 \quad$ Training Errors of Package Model $\ldots \ldots \ldots \ldots \ldots \ldots \ldots \ldots \ldots \ldots$

5.11 Training and Testing Results for Package Model ................ 99

5.12 Testing Error of Package Model with Three-Layer Perceptrons $\ldots \ldots \quad 100$

6.1 Output Temperatures for Detailed and Sub-models and Corresponding Errors of GaN Model with Four-Port Configuration ...

6.2 Output Temperatures for Detailed and Sub-models and Corresponding Errors of GaN Model with Five-Port Configuration ...

6.3 Temperature Rise and Maximum Error for Linear and Non-linear Substrate of GaN Model

6.4 Output Temperatures for Detailed and Sub-models and Corresponding Errors for GaAs Model $\ldots \ldots \ldots \ldots \ldots \ldots \ldots \ldots \ldots$

6.5 Temperature Rise and Maximum Error for Linear and Non-linear Substrate of GaAs Model ........................... 
6.6 Output Temperatures for Detailed and Sub-models and Corresponding Errors for Single Package Model with Right-side BC .

6.7 Output Temperatures for Detailed and Two Sub-models and Corresponding Errors for Four-Package Model with Right-side BC ...

6.8 Output Temperatures for Detailed and Two Sub-models and Corresponding Errors for Four-Package Model with Back-side BC ...

6.9 Temperature Rise and Maximum Error for Linear and Non-linear Substrate of Package Model $\ldots \ldots \ldots \ldots \ldots \ldots \ldots \ldots \ldots \ldots \ldots$

6.10 Simulation Time and Number of Blocks for Full-model and Mixed-

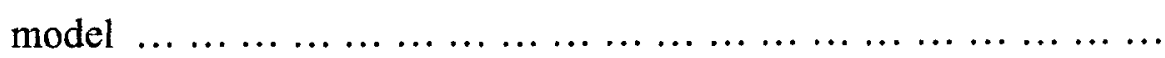

xii 


\section{List of Acronyms}

$\begin{array}{ll}\text { 3D } & \text { Three Dimensional } \\ \text { ANN } & \text { Artificial Neural Network } \\ \text { BC } & \text { Boundary Condition } \\ \text { BCI } & \text { Boundary Condition Independent } \\ \text { BGA } & \text { Ball Grid Array } \\ \text { BP } & \text { Back Propagation } \\ \text { CBGA } & \text { Ceramic Ball Grid Array } \\ \text { CG } & \text { Conjugate Gradient } \\ \text { FET } & \text { Field Effect Transistor } \\ \text { GaAs } & \text { Gallium Arsenide } \\ \text { GaN } & \text { Gallium Nitride } \\ \text { IC } & \text { Integrated Circuit } \\ \text { I/O } & \text { Input/Output } \\ \text { MLP } & \text { Multi Layer Perceptrons } \\ \text { PCB } & \text { Printed Circuit Board } \\ \text { RBF } & \text { Radial Basis Function } \\ \text { SiC } & \text { Silicon Carbide } \\ \text { SOM } & \text { Self Organizing Map } \\ \text { SOR } & \text { Successive Over Relaxation } \\ \text { TLM } & \text { Transmission Line Matrix } \\ \text { VLSI } & \text { Very Large Scale Integration } \\ & \end{array}$




\section{List of Symbols}

$\begin{array}{ll}\Delta T & \text { Temperature Rise } \\ V & \text { Voltage } \\ P & \text { Heat-flow (Power) } \\ I & \text { Current } \\ Q & \text { Heat/Charge } \\ R & \text { Resistance } \\ G & \text { Conductance } \\ \rho & \text { Resistivity } \\ K & \text { Thermal Conductivity } \\ \sigma & \text { Electrical Conductivity } \\ C & \text { Thermal Capacity/Capacitance } \\ c_{p} & \text { Specific Heat } \\ \varepsilon & \text { Permittivity } \\ T_{j} & \text { Junction Temperature } \\ \theta_{j-a} & \text { Total Thermal Resistance } \\ T_{a} & \text { Ambient Temperature } \\ q & \text { Heat Transfer Rate } \\ \theta_{j-c} & \text { Thermal Resistance of the Junction to Case } \\ \theta_{c-a} & \text { Thermal Resistance of the Case/Leads to the Ambient Air } \\ T_{c} & \text { Case Temperature } \\ I^{2} R & \text { Power Loss } \\ g(x, y, z) & \text { Volumetric Heat Generation } \\ n_{p} & \text { Number of Ports } \\ & \end{array}$




\begin{tabular}{ll}
$T_{p}$ & Port Temperature \\
$I_{p}$ & Port Heat-flow \\
$F_{p}$ & Source Vector \\
$A_{p p}$ & Transformation Matrix \\
$\Re$ & Set of All Real Vectors \\
$w$ & Weight \\
$L$ & Total Number of Layers \\
$l$ & Layer \\
$N$ & Number of Neurons \\
$x$ & Input Neuron \\
$z$ & Hidden Neuron \\
$n$ & Total Number of Input Neurons \\
$i, j, k$ & Associated with Number of Inputs/Outputs \\
$\gamma_{s}$ & Weighted Sum \\
$\sigma()$. & Neuron Activation Function \\
$y$ & Output Neuron \\
$m$ & Total Number of Output Neurons \\
$d$ & Measured/Simulated Output \\
$T_{r}$ & Training Data \\
$E(w)$ & Training Error \\
$p$ & Number of Optimization \\
$\eta$ & Learning Rate \\
$\mu$ & Momentum Factor \\
$N_{w}$ & Number of Iterations \\
$g$ & Gradient \\
$h$ & Conjugate Direction \\
$H$ & Hessian Matrix \\
$\delta$ & Relative Testing Error \\
\hline &
\end{tabular}


$T_{e}$ $M$

$C_{\text {coeff. }}$

$\alpha$
Test Data

Maximum Error

Correlation Coefficient

Heat Flow

xvi 


\section{Chapter 1}

\section{Introduction}

This chapter starts with an overview of thermal modeling, describes the motivations behind the research work carried out in this thesis and presents the thesis methodology. Finally, it outlines the organization of the thesis.

\subsection{Background}

The recent trend in IC technologies is to develop smaller devices with higher packaging density, faster speed and improved performance. Higher levels of IC integration and faster clock speeds result in higher power consumption. With the shrinkage of device dimensions, the complexities in chips increase. Consequently, faster switching speeds and greater on-chip functionality lead to larger heat generation and significant nonuniformities in on-chip heat flux of electronic devices.

The major part of the failures of integrated electronic components is temperature related.

The failure rate of microelectronic devices approximately doubles for every $10^{\circ} \mathrm{C}$ increase in temperature [1]. As a result, hot spots due to power dissipation have become 
a major long term reliability concern. For high temperatures and harsh environment applications, the most critical issues [2] for designing electronic packages for micro systems are: (1) Mitigation of thermal stress caused by the thermal expansion mismatches between the devices and various package elements including substrates (2) Thermal shock resistance brought about by thermal cycling during the services (3) Heat dissipation in order to keep the temperatures at safe operation levels.

With the development of smaller, lighter, faster as well as more functional electronic components, the reliability issue of these components becomes one of the major concerns in electronic packaging. Specifically, the performance and the reliability of electronic systems strongly depend on the junction temperature of the electronic components of the system. Hence, the determination of the component junction temperature is of utmost importance in thermal analysis of an electronic system.

Increased power dissipation and higher packaging density in modern electronic system require efficient and intelligent design and thermal management strategies to ensure the reliability of electronic products. To optimize long term reliability and performance, it has become essential to perform thermal and electro-thermal modeling and simulation prior to chip fabrication. Thermal management of electronic components plays an important role in all levels of packaging, i.e., from chip to package and system level. Currently, thermal modeling at system level is carried out for the analysis of parametric trends; but the predictive capabilities of these models are limited in most cases [3]. It is important to develop a model that is able to detect all the operating temperatures of 
various components in order to properly design the device or circuit layout and optimize thermal characteristics. The method should be fast, accurate and able to predict all critical temperatures.

\subsection{Motivation}

A common approach for thermal modeling is to utilize numerical modeling based on finite difference or other numerical techniques. A physical model based on this approach should be able to represent all the temperatures of interest of the original detailed structure. A large thermal model consisting of a die, package and board could have more than 500,000 blocks. A number of issues [4] are involved in such a large thermal package simulation: (1) Very long simulation time for both steady-state and transient (2) Nonlinear effects due to a temperature dependent thermal conductivity (3) The need to predict the multiple junction temperatures in multi-chip modules or detailed temperature distributions for some applications, such as, in optical devices (4) The need to optimize device layout/package geometries which requires many simulations. For these reasons, it is necessary to develop an efficient thermal model that can accurately predict multiple temperatures and represent the package in a detailed manner.

The use of sub-models or macro-models is a very useful approach for thermal analysis of linear models. A detailed model [5], which is a discretized version of the physical model, can be created by using a numerical simulator, such as, Atar [6-8]. This model of the component is then used in order to create a sub-model. The use of sub-model allows 
replacement of the complex parts of a large detailed model with a simple mathematical relationship. In order to create a sub-model it is needed to extract this relationship from the detailed model. This sub-model - linked to a detailed numerical model called the base-model through thermal ports - exchanges heat-flows with the base-model. The extraction of a linear sub-model can be performed straightforwardly in a number of ways [4].

Non-linear thermal modeling using sub-models requires a more general approach. The main challenge in non-linear component-modeling is to find the non-linear relationship between the port temperatures and port heat-flows of the sub-model. Precise mathematical modeling of such system is a very difficult task. Consequently, there is a need for an efficient modeling technique that can accurately represent the non-linear thermal behavior of the original model. Neural network-based modeling, a powerful technique that is capable of representing any non-linear relationship, can be applied to build a non-linear model of such system. However, the application of neural networks in thermal modeling has been left mostly unexplored so far by the research community [9]. The objective of this thesis is to investigate the neural network-based thermal modeling of electronic packages and to evaluate the performance of such models using a modeling tool called Neuromodeler. 


\subsection{Thesis Methodology}

As indicated in Section 1.2, the neural network modeling technique can be applied to perform a non-linear thermal analysis of electronic packages/components. In this thesis, the non-linear thermal analysis has been performed on three different thermal models as follows: (a) A Gallium Nitride (GaN) on SiC power package amplifier, (b) A Gallium Arsenide (GaAs) based power amplifier and (c) Ceramic-Ball-Grid-Array (CBGA) single-chip package. The steps followed in order to perform the non-linear thermal analysis have been:

1. Selection of the number of inputs for neural network modeling has been based on the linear simulation of the three above mentioned models using a thermal simulator called Atar.

2. Generation of data for training the neural network model. The data has been generated through non-linear simulation of Atar.

3. Development of a neural network model - using a modeling tool called Neuromodeler - through a process called training where the model learns the original input-output relationships. After the model is trained with a set of training data, it can become a fast and accurate model of the original problem.

4. Evaluation of the quality of the trained neural model through a process called testing using a set of data called testing data. In this thesis, the accuracy of the trained neural model has been evaluated by the average percentage error of the neural model output with respect to the test data. 
5. Full simulation of a mixed-model comprised of a detailed base-model and neuralnetwork based sub-models.

\subsection{Electro-Thermal Analogy}

A thermal system is found to be analogous to an electrical system if they have similar equations and boundary conditions. The equations that describe the behaviors of an electrical system can be transformed into the equations for the analogous thermal system by simply changing the variables. The analogy between thermal and electrical systems allows one to predict the thermal characteristics with widely known basic laws and methods for electrical simulation and modeling. Since Atar represents the final thermal model as a network of resistors and capacitors it is useful to know the thermal-electrical analogy for intuitive understanding. The thermal-electrical analogies are provided in

Table 1.1.

Table 1.1: An Analogy between Thermal and Electrical Quantities

\begin{tabular}{|c|l|c|c|c|c|}
\hline Thermal Quantities & & Units & Electrical Quantities & & Units \\
\hline Temperature Rise & $\Delta T$ & $\mathrm{~K}$ & Voltage & $V$ & $\mathrm{~V}$ \\
Heat-flow (Power) & $P$ & $\mathrm{~W}$ & Current & $I$ & $\mathrm{~A}$ \\
Heat & $Q$ & $\mathrm{~J}=\mathrm{W} . \mathrm{s}$ & Charge & $Q$ & $\mathrm{C}=\mathrm{A} . \mathrm{s}$ \\
Thermal Resistance & $R$ & $\mathrm{~K} / \mathrm{W}$ & Resistance & $R$ & $\Omega=\mathrm{V} / \mathrm{A}$ \\
Thermal Conductance & $G$ & $\mathrm{~W} / \mathrm{K}$ & Conductance & $G$ & $\mathrm{~S}=\Omega^{-1}$ \\
Thermal Resistivity & $\rho$ & $\mathrm{K} . \mathrm{m} / \mathrm{W}$ & Electrical Resistivity & $\rho$ & $\Omega . \mathrm{m}$ \\
Thermal Conductivity & $K$ & $\mathrm{~W} / \mathrm{K} . \mathrm{m}$ & Electrical Conductivity & $\sigma$ & $\mathrm{S} / \mathrm{m}$ \\
Heat Capacity & $C$ & J/K & Capacitance & $C$ & $\mathrm{~F}=\mathrm{C} / \mathrm{V}$ \\
Specific Heat & $c_{p}$ & $\mathrm{~J} / \mathrm{kg} . \mathrm{K}$ & Permittivity & $\varepsilon$ & $\mathrm{F} / \mathrm{m}$ \\
\hline
\end{tabular}




\subsection{Organization of the Thesis}

In this thesis, a non-linear thermal modeling based on neural network approach is presented. In this chapter, the problem domain of the thesis work has been described and the proposed approach has been outlined. The remaining part of the thesis is organized as follows:

- Chapter 2 provides a brief introduction of packaging along with various thermal issues in packaging. It also discusses simulation and modeling approaches involved in thermal packaging.

- Chapter 3 presents the basic concepts of the neural network-based modeling approach. It also discusses various well known neural network structures. Finally, it discusses the training algorithms as well as the testing of neural network models.

- Chapter 4 presents the linear simulations performed using Atar in order to determine the number of inputs for neural network modeling.

- In Chapter 5, a neural model is developed using Neuromodeler for each of the three models mentioned in Section 1.3. The qualities of the developed models are evaluated.

- In chapter 6 , the use of neural network in thermal modeling is presented for mixed-models.

- In Chapter 7, conclusions and future research directions are stated. 


\section{Chapter 2}

\section{Thermal Issues in Packaging}

This chapter starts with a brief introduction of electronic packaging and presents a review of thermal issues - one of the most important aspects of packaging. It also discusses the associated non-linear thermal equation. Finally, it provides a brief summary on simulation and modeling of thermal packaging.

\subsection{Introduction to Packaging}

With the rapid development of very large scale integration (VLSI) technologies, millions of electronic components with more functions and higher speeds are being fabricated on a single integrated circuit (IC) chip. As the circuit density on the chip increases, the speed of the tasks it performs also increases. To perform its functions, the IC chip communicates with other IC chips in a circuit through an input/output (I/O) system of interconnects. Packaging focuses on how these chips along with their embedded circuitry can be assembled cost effectively and with increased reliability. 
The need for miniaturization, higher reliability and competitive pricing has pushed product design far beyond the limitations of the conventional technology and has necessitated the development of new packaging technologies in order to insure that the final product meets the severe operational environments, cost restrictions and reliability standards of the industry. Packaging of electronic systems, in general, refers to the placement and connection of many electronic and electromechanical components in an enclosure in order to protect the system from the hostile environment and provide easy access for routine maintenance [10]. Based on the science of establishing interconnections, packaging encompasses the group of chip carriers or modules, printed circuit cards and boards, and cabling and connectors that make up an electronic assembly [11]. Starting from a single chip, which has been fabricated on a silicon wafer; the packaging process involves different stages [10] as shown in Figure 2.1. These stages are discussed briefly in the subsequent discussion.

First Level of Interconnections: Chip is housed and connected electrically to the carrier, often called a module, through some small wires.

Second Level of Interconnections: Chip carriers are placed on the circuit board and connected together with wiring traces formed by photo-etching the circuit board.

Third level of Interconnections: Connectors on the circuit boards are then inserted into contacts on a back panel. This back panel carries higher level of connections to communicate from one circuit board to other. Finally, the entire array of circuit boards, back panel, power supplies and cables are housed in a cabinet. 


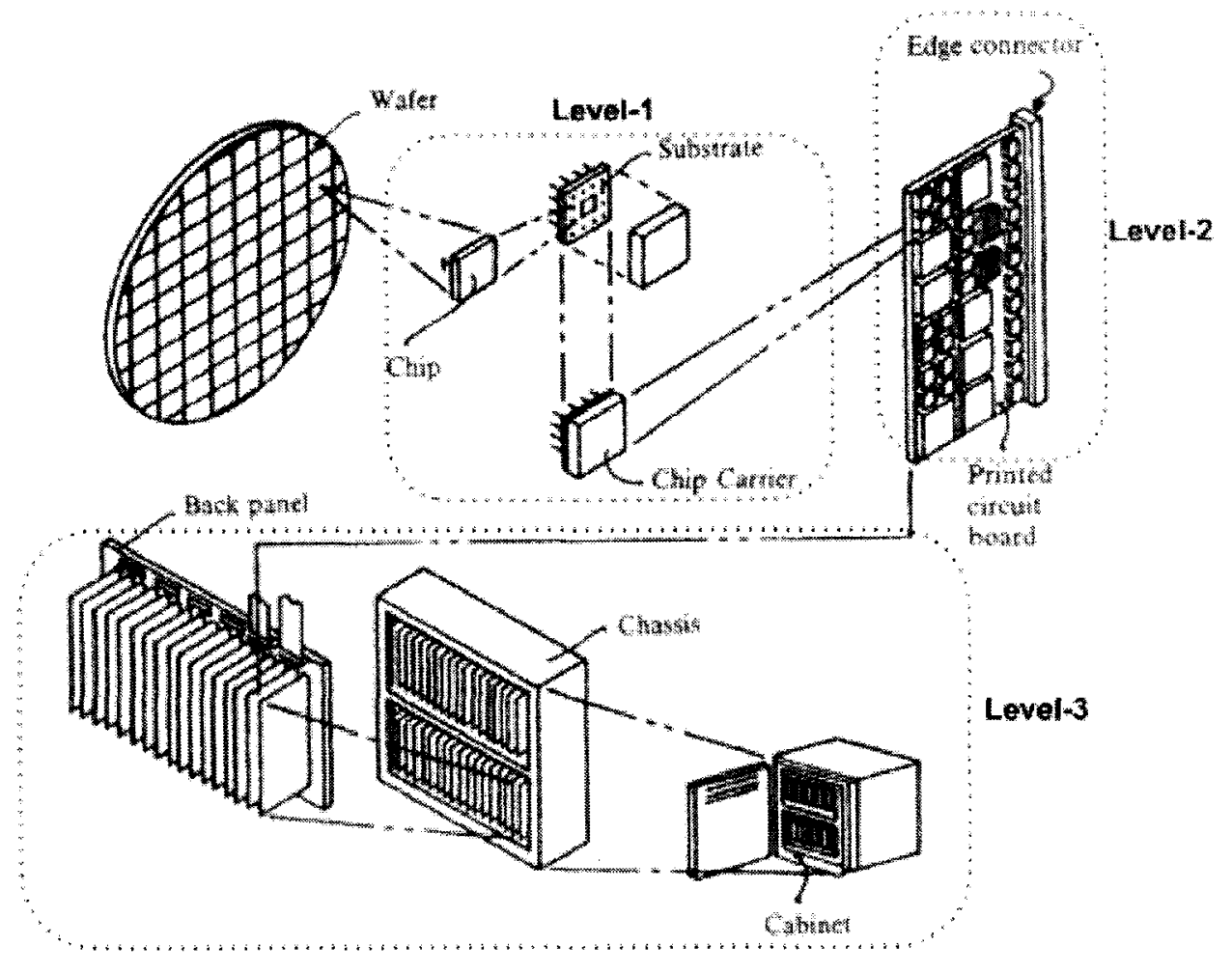

Figure 2.1: A Schematic Illustration of Several Levels of Packaging Involved in an Electronic System [10]

Electronic packaging will play a critical role in the next generation of semiconductor technology. The packaging aspect of the design of an electronic system is extremely important because about one-half of the cost of a system is involved in packaging [10]. Packaging provides a variety of mechanical, thermal and electrical functions. The major goals $[10-12]$ of the electronics packaging are:

- Power Distribution: To provide a mechanism for delivering power to the semiconductor circuits with a high degree of stability and free of electrical noise, which can arise when many digital circuits switch simultaneously. 
- Signal Distribution: To carry electrical signals from one circuit to another, while maintaining fidelity of the signal even at the highest speeds at which the circuitry can operate.

- Thermal Management: To maintain internal product/system and device temperature by removing the excess heat generated by the circuits on the IC chip.

- Protection: In addition to carefully packing thousands of electronic components into a stylish and functional cabinet, packaging protects these components and the circuitry from hostile environments as well as from abusive handling.

The thermal aspects of packaging are discussed in more detail in the following section.

\subsection{Thermal Packaging}

The diverse applications of electronic products and the recent acceleration of packaging technology are creating new challenges to the thermal design. Placing more functions in a smaller package results in higher heat densities. Higher operating temperatures accelerate various failure mechanisms, such as, creep, corrosion, electro-migration etc. [11]. Large fluctuations or variations of temperature causes malfunction or even breakdown of the equipment. In order to protect the chip and to keep it running at its design temperature, the excess heat needs to be dissipated. Since the operating temperature of the chips markedly affects the reliability of the circuit board, system availability and serviceability 
of electronic equipments, heat management plays one of the most important roles in electronic packaging.

Thermal management of electronic packaging is an all-inclusive method that involves the selection, analysis, testing, and verification of a cooling design in order to produce a reliable end product [13]. With the very large number of components used in a typical electronic system, it is necessary to minimize the junction temperatures $\left(T_{j}\right)$ of the most critical of the components for enhancing the reliability and improving the availability of the system. Consequently, the goal of thermal management is to move the heat out of the junctions of power-dissipating devices while minimizing cost, noise and overall complexity and size of the heat-removing devices [14].

Thermal analysis of electronic system involves the prediction of the junction temperature of the integrated circuits. The point, at which the maximum temperature of a chip is reached, is considered as the junction temperature. The main purpose of thermal packaging is to create and maintain a temperature distribution with limited variations around a moderate level across the transistor junctions by placing the heat generating components in the proper location in a package and by measuring the heat that needs to be disposed to the exterior of the equipment. The trend to increase chip power dissipation has become a major concern for the circuit designers and places significant emphasis on thermal design considerations at all levels of electronic packaging, i.e., from the chip level to the system level [11]. 
The heat transfer characteristics of an IC package is usually specified in terms of its total thermal resistance $\theta_{j-a}\left({ }^{\circ} \mathrm{C} / \mathrm{W}\right)$, which refers to the temperature difference between the junction temperature $T_{j}\left({ }^{\circ} \mathrm{C}\right)$ of the electronic device and the ambient temperature $T_{a}$ $\left({ }^{\circ} \mathrm{C}\right)$ of the air flowing over the chip carrier $[10,13]$. The temperature difference is,

$$
T_{j}-T_{a}=q \theta_{j-a}
$$

$\theta_{j-a}$ is defined as,

$$
\theta_{j-a}=\theta_{j-c}+\theta_{c-a}
$$

where, $\theta_{j-c}\left({ }^{\circ} \mathrm{C} / \mathrm{W}\right)$ is the thermal resistance of the junction to case and $\theta_{c-a}\left({ }^{\circ} \mathrm{C} / \mathrm{W}\right)$ is the thermal resistance of the case/leads to the ambient air and can be expressed as,

$$
\begin{gathered}
\theta_{j-c}=\frac{T_{j}-T_{c}}{q} \\
\theta_{c-a}=\frac{T_{c}-T_{a}}{q}
\end{gathered}
$$

where, $T_{c}\left({ }^{\circ} \mathrm{C}\right)$ is the case temperature and $q\left(\mathrm{~W} / \mathrm{m}^{3}\right)$ is the heat transfer rate per unit volume. The resistance $\theta_{j-c}$ is due to conduction from the chip to the package and it involves the detailed design of the chip carrier. The resistance $\theta_{c-a}$ is due to convection and formation of a boundary layer between the case and its leads and the air. 


\subsection{Thermal Equation for Conduction}

Heat is generated in electronic devices primarily by $I^{2} R$ losses and must be dissipated in order to avoid excessive temperature that can impair the reliability of the system. The amount of heat generated varies from a few milliwatts (for example, in a wristwatch) to $10 \mathrm{~kW}$ or more (for example, in a large mainframe computer) depending on the size and complexity of the system. The design for dissipating the heat generated depends to a significant degree on the type of the product, the environment, the accessibility of a coolant, the reliability and the system availability requirements and also the cost involved in providing an adequate cooling system.

Heat is transferred from the component (source) to the environment (sink) by three basic mechanisms, which include conduction, convection and radiation. This thesis is focused only towards conduction which is associated with $1^{\text {st }}$ and $2^{\text {nd }}$ level of packaging.

Conduction is the most important heat transfer mechanism at the chip and package level. Conduction is due to the energy exchange of rapidly vibrating molecules in a hotter region to less rapidly vibrating molecules in a cooler region. Conduction is usually associated with solids but it may also occur in fluids (liquids and gases) over very short distances. The heat-flow from the junction of a chip in an IC package to its case is a common example of conduction [13]. 
The following partial differential equation [15] needs to be solved in order to solve the conductive heat-flow in an IC or package structure.

$$
\nabla(K(T) \nabla T)+g(x, y, z)=0
$$

where, $K(T)(\mathrm{W} / \mathrm{m} . \mathrm{K})$ is the temperature dependent thermal conductivity and $g(x, y, z)$ (W) is the volumetric heat generation. This is a steady state equation of heat conduction and allows calculating the temperature distribution in a structure containing heat sources having known boundary conditions.

For Si and with a temperature rise of $\Delta T<100^{\circ} \mathrm{C}, K \sim K_{300 K}$, i.e., the Equation (2.5) is essentially linear. However, for III-V materials and a temperature rise of $\Delta T>75^{\circ} \mathrm{C}$, the temperature dependent thermal conductivity makes the Equation (2.5) non-linear. This temperature dependant nature of $K(T)$ is an important issue for power amplifiers and optical modules. This thesis is mainly focused towards the non-linear thermal behavior of an electronic package.

Due to the complex geometries and non-linear nature of the heat conduction equation in (2.5), it is very difficult to analytically solve the equation. To obtain solution for realistic packages, an appropriate discretized 3D model must be built in order to produce a numerical solution of the equation. The thermal simulator Atar described in the subsequent section of this thesis can be used to solve this non-linear equation. 


\subsection{Package Simulation and Modeling}

Packaging technology increases the performance of electronic systems and subsystems while reducing their size. The thermal performance of packaging is typically predicted by two methods,

- Package modeling using simplified models of the physical heat-flow through geometry. These models are generally linear and produce an approximate junction temperature.

- Package simulation using a numerical thermal simulator. Simulation involves a detailed numerical simulation incorporating complex geometry and possibly non-linear effects. Typical methods are finite elements and finite difference. Simplified package models are often derived from simulations [5].

In the subsequent sections, a numerical tool for package simulation called Atar and the use of sub-models in thermal packaging is discussed. Finally, a neural network-based modeling tool called Neuromodeler used in this thesis is discussed. 


\subsubsection{Thermal Simulator-Atar}

A common approach of simulation is to write computer codes that provide numerical solutions to the specified problems with complex geometries arising in electronic packaging. Atar $[6-8]$ is a thermal simulator tool based on this approach. This tool was developed by the modeling group at Carleton University and has been used in this thesis for simulation purpose. It is a three-dimensional (3D) thermal simulation tool for semiconductor integrated devices, packages and assemblies. The simulation tool uses a technology description and layout information in order to automatically generate a full 3D physical model, complete with discretization, and then solves for the temperature distribution. The simulator automatically produces an accurate 3D model of the device from layout information which is appropriately meshed for solving the non-linear thermal diffusion equation that describes the heat-flow. A 3D model represents both the substrate of the device/package and any metallization that is present. A 3D quad tree mesh is created that produces a fine mesh at the heat generation region of the device, but still uses a moderate number of temperature nodes for the entire device. From this model, a network of thermal resistors and capacitors are extracted that mathematically represents the model which can be solved to obtain the temperature distribution. It is worthwhile to mention that for a non-linear model, the thermal resistors are temperature dependent.

Atar offers two steady state internal solvers. A direct solver sets up a global sparse array and solves the linear set of equations representing the thermal resistor network. The second solver uses a Successive Over Relaxation (SOR) technique of the nodal 
temperature equations and is particularly useful for large or non-linear problems with temperature dependent material properties or boundary conditions (BCs).

Atar also incorporates a transient solver based on a transmission line matrix (TLM) implementation using a physical extraction of a resistance and capacitance network. The formulation allows for temperature dependent material parameters and a non-uniform time stepping.

Atar uses a hierarchical model building technique. The hierarchical model building technique is based on the idea of component and sub-models. The next section provides a discussion of the use of sub-models in thermal packaging.

\subsubsection{Use of Sub-model in Thermal Modeling}

As mentioned in Chapter 1, number of issues are involved in a large thermal package simulation, such as, very long simulation time for both steady-state and transient, nonlinear effects due to a temperature dependent thermal conductivity, the need for prediction of the multiple junction temperatures in multi-chip modules or detailed temperature distributions for some applications, such as, in optical devices and also the need for optimizing the device layout/package geometries which requires many simulations. For these reasons, it is essential to develop an efficient thermal model to be able to accurately predict multiple temperatures and represent the package in a detailed manner. 
The approach taken in Atar to solve this problem is to break the original model into smaller models that are then "linked" together. These smaller models are originally fully detailed Atar models which are referred as either a base-model or a component-model. The base-model is typically the largest scale structure in the simulation, for example, the PC board. A component-model is a well defined part of the simulation, such as, a package or chip. Often components are repeated within a model. Atar allows the linking of base-model and component-models to form a complete hierarchical simulation. For example, linking four packages to a board. However, simply linking together of all the fully detailed models does not make simulation times smaller as the number of elements in the model is the same as for single model of the entire structure; it only facilitates the building process. Figure 2.2 shows how sub-model is linked to a base-model of a mixedmodel.

To reduce simulation time the component-models must be replaced by a mathematically equivalent model which eliminates the need to incorporate the component-model. This mathematical representation of a component-model is referred as a sub-model. Submodels, which are linked to a base-model through thermal ports, exchange heat-flows with the base-model. Well defined multiple ports must be utilized for an accurate representation of the thermal behavior of a component-model. By linking sub-models with a base-model a mixed-model is formed. A thermal simulation of such a model involves a detailed numerical model, the base-model linked to sub-models through thermal ports where the linking size of a sub-model is $n_{p} \times n_{p}$, where $n_{p}$ denotes the 
number of ports. The accuracy and determination of the internal temperatures of the submodel depend on the geometry of the ports. The ports are characterized by two parameters: a port temperature $\vec{T}_{p}$ and port heat-flow $\vec{I}_{p}$ that are analogous to port voltage and port current respectively in an interconnected electrical sub-network.

For a linear model, the thermal equations representing the component-model can be generated by Atar. If the BCs and thermal ports of the component-model are explicitly described, the linear relationship between the port heat-flows and temperatures can be characterized by Equation (2.6).

$$
\vec{I}_{p}=\vec{F}_{p}+G_{p} \vec{T}_{p}
$$

where, $\vec{F}_{p} \in \mathfrak{R}^{n_{p} \times 1}$ is the source vector representing internal power generation and applied boundary conditions, $G_{p} \in \mathfrak{R}^{n_{p} \times n_{p}}$ is the discretized thermal conductance matrix, $\vec{T}_{p} \in \mathfrak{R}^{n_{p} \times 1}$ is the unknown vector of port temperatures in the system and $p$ denotes the port. Atar allows for the connection and linking of a sub-model described by (2.6) to a detailed base-model and then solution of the mixed-model. The solution will produce all the internal temperatures of the base-model, the port temperatures and the internal temperatures of interest for the component-model. In the case where the model is linear, as in Equation (2.6), model reduction techniques can be used to capture the behavior of a large model by creating a small, fast reduced sub-model which is parameterized for external BCs. This is shown schematically in Figure 2.2 (b). 


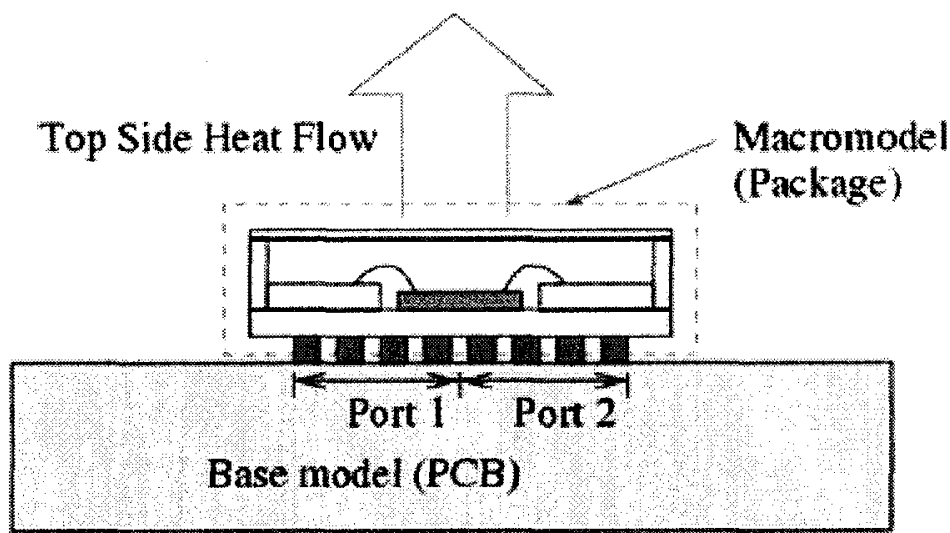

Figure 2.2 (a): Schematic Representation of a Mixed-Model Including a Two-port Sub-model Attached to a Base-Model (PCB) [4]

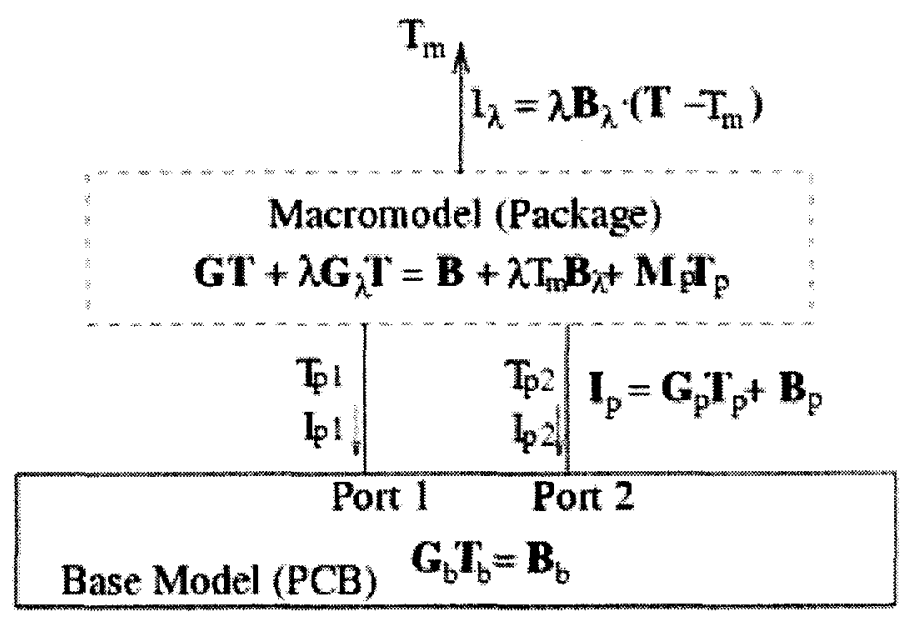

Figure 2.2 (b): Schematic Representation of Relationship between Components in a Mixed-model Using a Linear Sub-model [4] 
The sub-modeling approach described above is very useful for linear thermal models; however, non-linear models (where $K$ is a function of $T$ ) require a more general approach. For the non-linear case, the port heat-flow can be represented by Equation (2.7).

$$
\vec{I}_{p}=f\left(\vec{T}_{p}\right)
$$

where, $f$ is a non-linear function. The first problem with a non-linear component is to find the non-linear relationship between the port temperatures and port heat-flows. The second issue that arises is that the mixed-model is now non-linear and it is more difficult to solve such system. However, as we shall see, neural network technique can be applied to build a non-linear model of such system and the solution of the mixed-model can then be done by iteration. In the next section, a neural network-based modeling tool, called Neuromodeler, which is used in this thesis, is described.

\subsubsection{Thermal Modeling Tool - Neuromodeler}

In this thesis, a neural network-based modeling tool, Neuromodeler [16], is used to analyze the non-linear behavior of thermal models. Neuromodeler, developed in Carleton University, is a unique software tool. It allows quick development of neural network models for active and passive components at both device and circuit-levels for linear or non-linear simulations. Models developed by Neuromodeler are much faster than original 
detailed models, more accurate and flexible than empirical models. The neural networks technique used in this tool are discussed in detail in Chapter 3.

The inputs for Neuromodeler in this thesis are the port temperatures $\left(T_{p}\right.$ 's), input power $(P)$ and boundary conditions $(B C ' s)$ applied to the sub-model. Outputs are the port heatflows ( $I_{p}$ 's) and temperatures of interest $\left(T_{j}\right.$ 's). A thermal model is developed through a process called training. The performance of this model is evaluated by testing it with a set of testing data. The MATLAB/C code obtained from the Neuromodeler is used to link the sub-model with the base-model.

\subsection{Summary}

In this chapter, a review of packaging with an emphasis on the thermal packaging has been presented. A brief discussion on simulation and modeling has been provided. In the next chapter, a review on the neural network-based modeling approach that has been used in this thesis is presented. 


\section{Chapter 3}

\section{Neural Networks}

This chapter presents the basic concepts on neural network-based modeling approach where the model can be developed by learning from the available data obtained from the original system. Some widely-used neural network structures are discussed. Finally, a brief discussion on the training algorithms and various quality measures involved in neural network testing are provided.

\subsection{Introduction to Neural Networks}

Neural networks, also called artificial neural networks (ANN), have been widely applied to various fields because of their ability to solve cumbersome or intractable problems by learning directly from data. Neural network is an information processing system inspired by the ability of the human brain to learn from observations and to generalize by abstraction [17]. In other words, as in [18], it is a massively parallel distributed processor made up of simple processing units which has a natural propensity of storing experiential knowledge and making it available for use. Neural networks, being able to learn and generalize from data even when component formulas are not available, allow reuse of the 
same modeling technology for both linear and non-linear problems and at both device and circuit levels.

ANN is considered to be a powerful technique for modeling general input-output relationships. Models are provided with sufficient training data from which it learns the underlying input/output mapping. The main characteristics $[17,18]$ of ANN are:

Input-Output Mapping: Neural networks are capable of learning the non-linear behaviors directly from measured or simulated input-output data of the original circuit; consequently, manual effort of developing equivalent-circuit topology can be avoided [19]. Neural networks do not require any prior knowledge of input-output mapping for modeling and can easily infer the unknown input-output relationships from the training data provided to the network. As a result, the fitted function can be accurately represented by an ANN even if it is not explicitly defined.

Generalization: It is the ability of a network to estimate the output accurately when presented with the input that is never used during training. Neural networks have good generalization capability since they can respond correctly to new data that has not been used for model development.

Non-linearity: Neural networks are able to model highly non-linear as well as linear input-output mappings. They are capable of forming an arbitrarily close approximation to any continuous non-linear mapping [20]. 


\subsection{Neural Network Structures}

A general theory of information processing based on networks of binary switching or decision elements, which are somewhat euphemistically called neurons was proposed by McCulloch and Pitts [21]. A neural network model, also called connectionist network, consists of at least two physical components: (1) the processing elements, called neurons (2) connections between the neurons, known as the links. Every link in the network is associated with a weight parameter $\vec{w}$ that is adjusted during training process so that the error between the neural model outputs and the original problem outputs is minimized. Since the links and their weights determine the behavior of a model, they are considered as the important parameters of any model.

Neurons, receiving stimuli from outside the network (i.e., not from neurons of the network), are called input neurons while the neurons having no link leading away from them and whose outputs are used externally are called output neurons. Hidden neurons are the neurons that receive stimuli from other neurons and whose output is a stimulus for other neurons in the network.

To determine the appropriate number of hidden neurons required for a modeling task is still a topic of ongoing research. Depending on the degree of non-linearity and the dimensionality of the original problem, an appropriate number of hidden neurons in a network is chosen. For example, highly non-linear problems need more hidden neurons while smoother problem needs fewer neurons $[17,22]$. Since the number of hidden 
neurons gives sufficient freedom to the network to accurately learn the problem behavior and to capture the problem non-linearity, too many or insufficient number of hidden neurons may cause over-learning or under-learning respectively.

Neurons are connected to one another in different ways. After receiving stimulus from the neighboring neurons, each neuron (except the input neurons) processes the information and produces an output. Information can be processed by a neuron in various ways. The manner in which the output is computed from the input and the definition of the weight parameter $\vec{w}$ determine the structure of neural network. Different neural network structures are used depending on the application. Multi Layer Perceptrons (MLP), Radial Basis Functions (RBF) Networks, Wavelet Neural Networks, Recurrent Neural Networks, Knowledge Based Neural Networks are some examples of different neural network structures. Neural network structure may differ in accuracy and efficiency. In fact, selection of right type of structure appropriate for a particular application may give better accuracy while having less training data. The significant features [17] of neural network structures are:

- Neural networks are distributed models by nature. No single neuron is capable of producing the overall input-output relationship; the combination of many neurons can produce this relationship. Each neuron is a simple processing element having a switching activation function. This rich combination of the neuron switching states responding to different values of external stimuli enables the network to represent a non-linear input-output mapping. 
- Neural networks can be trained to represent any given problem behavior because of their powerful learning capability. Neural networks with different sets of weighted connections are capable of representing a diverse range of input-output mapping problems by capturing/encoding the problem information from the raw training data.

In the subsequent sections, some widely-used neural network structures, with an emphasis on MLP which has been used in this thesis, are discussed.

\subsubsection{Multi Layer Perceptrons}

An extensive study on a specific type of neural network, called a perceptron, was conducted by Rosenblatt and his collaborators [23]. The flow of information in this neural network structure is strictly directional - from input neurons to output neurons. This idea led to a basic type of neural networks, called feed-forward networks. Feedforward networks are capable of approximating generic classes of functions, including continuous and integrable functions $[17,24]$.

The Multi Layer Perceptrons (MLP) structure, belonging to feed-forward, is the most popular type of neural network since this structure and its training are well-established and the model has good generalization capability. It is considered to be the simplest structure capable of representing any Boolean function and of approximating more general functions to arbitrary precision [25]. An MLP structure consists of a set of sensory units (source nodes) that constitute the input layer (the first layer of the network), 
one or more hidden layers of computation nodes, and an output layer (the last layer of the network) of computation nodes as shown in Figure 3.1. Suppose the total number of layers is $L$. The $I^{\text {st }}$ layer is the input layer, the $L^{\text {th }}$ layer is the output layer and layers 2 to $(L-1)$ are hidden layers. Let the number of neurons in $l^{\text {th }}$ layer be $N_{l}, l=1,2, \ldots, L$.

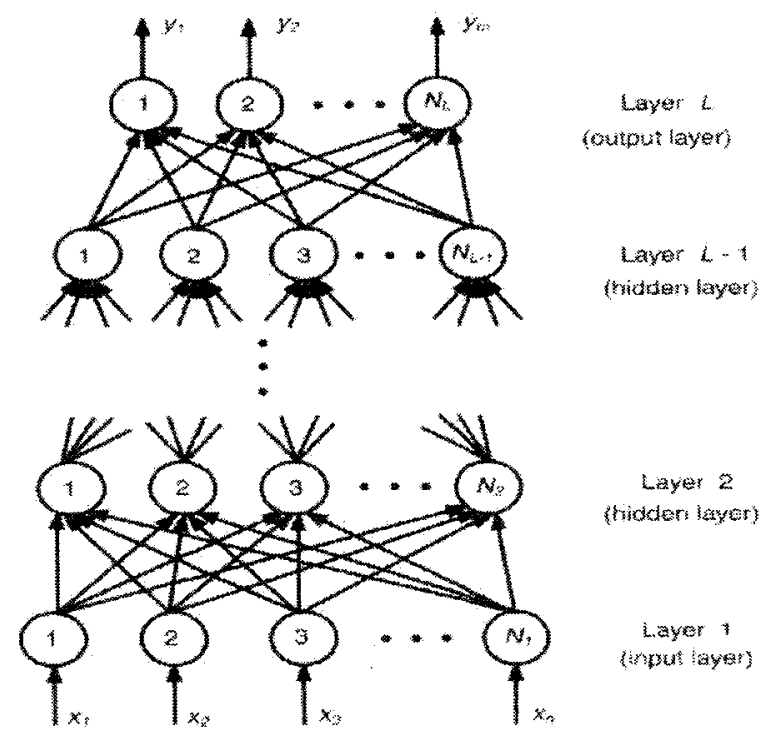

Figure 3.1: Multi Layer Perceptrons (MLP) Structure [17]

Let $w_{i j}^{l}$ represents the weight of the link between $j^{t h}$ neuron of $(l-1)^{t h}$ layer and $i^{\text {th }}$ neuron of the $l^{t h}$ layer, $1 \leq j \leq N_{l-1}, 1 \leq i \leq N_{l}$. Let $x_{i}$ represents the $i^{t h}$ external neuron to the MLP, and $z_{i}^{l}$ be the output of $i^{t h}$ neuron of $l^{t h}$ layer. Let $w_{i 0}^{l}$, an extra weight parameter for each neuron, represents the bias for $i^{\text {th }}$ neuron of $l^{\text {th }}$ layer. As such, $\vec{w}$ of MLP includes $w_{i j}^{l}, j=0,1, \ldots, N_{l-1}, i=1,2, \ldots, N_{l}, l=2,3, \ldots, L$, that is, 


$$
\vec{w}=\left[w_{10}^{2} w_{11}^{2} w_{12}^{2} \ldots, w_{N_{L}}^{L} N_{L-1}\right]^{T}
$$

In a neural network, each neuron - with the exception of input neurons - receives and processes stimuli (inputs) from other neurons and the processed information is available at the output end of the neuron. In order to understand how each neuron in an MLP processes the information, let $x_{i}$ represent the $i^{t h}$ external input to the MLP, and $z_{i}^{l}$ be the output of the $i^{\text {th }}$ neuron of $l^{\text {th }}$ layer. A neuron of the $l^{\text {th }}$ layer receives stimuli from the neurons of $(l-1)^{t h}$ layer, i.e., $z_{1}^{l-1}, z_{2}^{l-1}, \ldots, z_{N_{l-1}}^{l-1}$. Each input is first multiplied by the corresponding weight parameter and the resulting products are then added to produce a weighted sum $\gamma_{s}$. This weighted sum is passed through a neuron activation function $\sigma(\cdot)$ in order to produce the final output $z_{i}^{l}$. This final output $z_{i}^{l}$ becomes the stimulus for neuron in the next layer. The input neurons simply relay the external stimuli to the hidden layer neurons; i.e., the input neuron activation function is a relay function, $z_{i}^{l}=x_{i}, i=1,2, \ldots, n$ and $n=N_{1}$.

The activation function used in neural network can be logistic function, such as, sigmoid or simple linear functions that compute the weighted sum of the stimuli. In order to model continuous electrical parameters, linear activation functions are more suitable for output neurons. The use of linear activation functions in the output neurons helps to improve the numerical conditioning of the neural network training process. The linear activation function is defined as, 


$$
\sigma\left(\gamma_{s}\right)=\gamma_{s}=\sum_{j=0}^{N_{L-1}} w_{i j}^{L} z_{j}^{L-1}
$$

where, $w_{i j}^{L}$ represents the weight of the link between $j^{t h}$ neuron of the $(L-1)^{t h}$ layer and the $i^{\text {th }}$ neuron of the $L^{\text {th }}$ layer.

The most commonly used hidden neuron activation function is the sigmoid function given by,

$$
\sigma\left(\gamma_{s}\right)=\frac{1}{1+e^{-\gamma_{s}}}
$$

where, $\sigma\left(\gamma_{s}\right) \rightarrow 1$ as $\gamma_{s} \rightarrow+\infty$ and $\sigma\left(\gamma_{s}\right) \rightarrow 0$ as $\gamma_{s} \rightarrow-\infty$

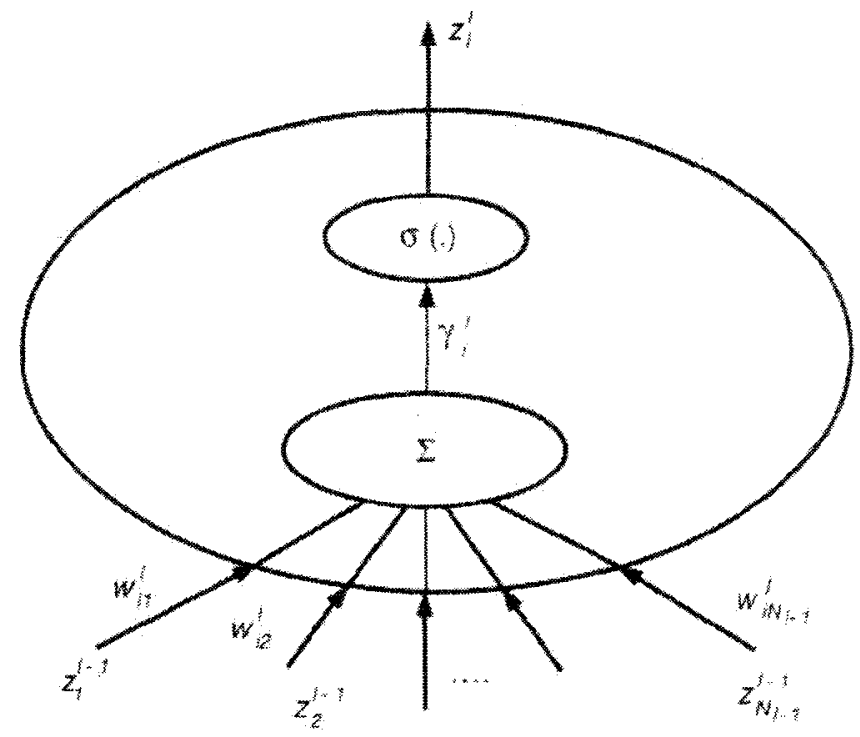

Figure 3.2: Information Processing by $i^{\text {th }}$ Neuron of $\boldsymbol{l}^{\text {th }}$ layer [17] 
Other possible hidden neuron activation functions are the arc-tangent function given by,

$$
\sigma\left(\gamma_{s}\right)=\frac{2}{\pi} \arctan \left(\gamma_{s}\right)
$$

and the hyperbolic tangent function given by,

$$
\sigma\left(\gamma_{s}\right)=\frac{e^{\gamma_{s}}-e^{-\gamma_{s}}}{e^{\gamma_{s}}+e^{-\gamma_{s}}}
$$

All of these logistic functions [17] mentioned above are bounded, continuous, monotonic and continuously differentiable.

Let, the inputs $\vec{x}=\left[x_{1} x_{2} \ldots x_{n}\right]^{T}$ and the weights $\vec{w}$ are used to compute the outputs $\vec{y}=\left[y_{1} y_{2} \ldots y_{m}\right]^{T}$ from an MLP neural network. In the feed-forward process, such as, in MLP, the external inputs are first fed to the input neurons ( $I^{\text {st }}$ layer). Then the outputs from the input neurons are fed to the hidden neurons of the $2^{\text {nd }}$ layer, and so on. Finally, the outputs of the $(L-1)^{t h}$ layer are fed to the output neurons of $L^{\text {th }}$ layer. The computation is represented as,

$$
\begin{gathered}
z_{i}^{l}=x_{i}, i=1,2, \ldots, N_{1}, \text { and } n=N_{1} \\
z_{i}^{l}=\sigma\left(\sum_{j=0}^{N_{l-1}} w_{i j}^{l} z_{j}^{l-1}\right), i=1,2, \ldots, N_{l}, l=2,3, \ldots, L
\end{gathered}
$$

The outputs of the neural network extracted from the output neurons are as follows, 


$$
y_{i}=z_{i}^{L}, i=1,2, \ldots, N_{L}, m=N_{L}
$$

The weights $\vec{w}$ are fixed during a feed-forward computation and these weights are determined through the training process.

According to the universal approximation theorem, there exists a three-layer MLP that approximates virtually any arbitrary non-linear, continuous, multi dimensional function with any desired accuracy [17]. MLP networks can construct global approximation to non-linear input-output mapping. Consequently, they are capable of generalizing in those regions of the input space where little or no training data is available. Moreover, neural networks with one hidden layer are necessary and sufficient for arbitrarily non-linear function approximation. In practice, neural networks with one or two hidden layers are more commonly used. The performance of a neural network can be evaluated in terms of generalization capability and mapping capability [26]. In the function approximation or regression area, generalization capability is a major concern. In such applications, threelayer perceptrons are usually preferred because of having fewer hidden neurons in the resulting network [27]. In other type of applications, where better mapping capability is necessary, four-layer perceptrons might be utilized [26]. In this thesis, both three-layer and four-layer perceptrons structures have been used for thermal package modeling. 


\subsubsection{Radial Basis Function Networks}

Feed-forward neural networks with an input layer, an output layer and a single hidden layer that use radial basis activation functions for hidden neurons are called Radial Basis Function $(R B F)$ networks [17]. Gaussian and multi-quadratic activation functions are more commonly used in RBF. RBF is suitable for problems with smaller number of inputs.

\subsubsection{Wavelet Neural Networks}

Wavelet neural network construction method is developed by using the theoretical features of the wavelet transform [17]. This method helps to determine the neural network parameters and the number of hidden neurons during training. Wavelet networks use wavelet function as hidden neuron activation function.

\subsubsection{Recurrent Neural Networks}

Recurrent neural network structure, having at least one feed-back loop, allows modeling the time-domain behavior of a dynamic system where the outputs of the system depend not only on the present inputs, but also on the history of the system states and inputs [17]. 


\subsection{Training of Neural Networks}

Development of a neural network model for a device - circuit is possible by learning and abstracting from measured - simulated data through a process called training [28]. It learns the original input-output relationship through training and does not represent any device - circuit behavior unless it is trained with corresponding device - circuit data. The purpose of training, which is the most time-consuming step in neural network model development, is to determine neural network integral weights $\vec{w}$ such that the neural model output best matches the training data. The neural network becomes a fast and accurate model of the original problem it has learnt after it is trained with sufficient data.

Training algorithm, which can be viewed as an optimization process for finding values of weights associated with various neurons, is an integral part of neural network model development. An appropriate structure may still fail to give a better model, unless trained by a suitable algorithm [28]. A good training algorithm is likely to shorten the training time and would help achieving a better accuracy. Various well known optimization techniques that are used to train neural network structures are: Back Propagation, Conjugate Gradient, Quasi-Newton, Levenberg-Marquardt, Gauss-Newton, Genetic Algorithms and Simulated Annealing.

The most challenging task in neural model development is to find an optimal set of weight parameter $\vec{w}$, so that the model output $\vec{y}(\vec{x}, \vec{w})$ closely approximates the original 
problem behavior. This is achieved through the training process (optimization in $\vec{w}-$ space) with a given set of training data. A good collection of well-distributed, sufficient and accurately measured - simulated training data is the basic requirement to obtain an accurate model [28]. The training data is denoted by the input-output sample pairs $\left\{\left(x_{k}, d_{k}\right), k \in T_{r}\right\}$, where $d_{k}$ is the measured/simulated output for $\vec{y}$ for the input $x_{k}$, and $T_{r}$ is the index set of training data. The measured/simulated outputs are related by $d_{k}=f\left(x_{k}\right)$. The training error $E(\vec{w})$ is defined as [17],

$$
E(\vec{w})=\sum_{k \in T_{r}} E_{k}(\vec{w})
$$

where, $E_{k}(\vec{w})$ is the error between neural network prediction and the $k^{\text {th }}$ training sample given by,

$$
E_{k}(\vec{w})=\frac{1}{p} \sum_{j=1}^{m}\left|y_{j}\left(x_{k}, \vec{w}\right)-d_{j k}\right|^{p}
$$

where, $\quad m$ is the dimension of the output $\vec{y}$, $p$ represents least $p^{\text {th }}$ optimization, $d_{j k}$ is the $j^{\text {th }}$ element of $d_{k}$, and $y_{j}\left(x_{k}, \vec{w}\right)$ is the $j^{t h}$ output of the neural network for input sample $x_{k}$. 
The primary objective of the training process is to determine the weight parameters $\vec{w}$ such that the error $E(\vec{w})$ is minimized.

The normalized training error [17] is defined as,

$$
E_{T_{r}}(\vec{w})=\left[\frac{1}{\operatorname{size}\left(T_{r}\right) \cdot m} \sum_{k \in T_{r}} \sum_{j=1}^{m}\left|\frac{y_{j}\left(x_{k}, \vec{w}\right)-d_{j k}}{d_{\max , j}-d_{\min , j}}\right|^{p}\right]^{\frac{1}{p}}
$$

where, $d_{\max , j}$ and $d_{\min , j}$ are the maximum and minimum values of the $j^{\text {th }}$ element of all vectors of $d_{k}, k \in T_{r}$.

The training process can be classified into two groups, namely, supervised training and unsupervised training. The training, in which both the input data $(\vec{x})$ and output data $(\vec{y})$ are used to train the neural network, for example, in MLP training, is called supervised training. On the other hand, in unsupervised training, either the input data $(\vec{x})$ or the output data $(\vec{y})$ is used for training, as in self-organizing map (SOM) training. 


\subsubsection{Back Propagation}

Rumelhart, Hinton and Williams [29] proposed a systematic neural network training approach and one of the significant contributions of their work is the error Back Propagation (BP) algorithm. BP, belonging to supervised learning, is considered as a computationally efficient method for the training of Multi Layer Perceptrons [18].

The first step of training is to initialize the weight parameter $\vec{w}$ by using small random values. The weight parameter $\vec{w}$ is updated during training along the negative direction of the gradient of the training error $E$, as $\vec{w}=\vec{w}-\eta\left(\frac{\partial E}{\partial \vec{w}}\right)$ until $E$ becomes small enough. Here, the parameter $\eta$ is called the learning rate. In the case where just one training sample is used at a time to update $\vec{w}$, a per-sample error function $E_{k}[17]$ given by,

$$
E_{k}=\frac{1}{2} \sum_{j=1}^{m}\left\{y_{j}\left(x_{k}, \vec{w}\right)-d_{j k}\right\}^{2}
$$

is used and $\vec{w}$ is updated as $\vec{w}=\vec{w}-\eta\left(\frac{\partial E_{k}}{\partial \vec{w}}\right)$.

Back propagation is a stochastic algorithm based upon the steepest descent principle [30]. In this case, the weights of the neural network are updated along the negative gradient direction in the weight space. The updated equations are given by, 


$$
\Delta \vec{w}_{\text {now }}=\vec{w}_{\text {next }}-\vec{w}_{\text {now }}=-\left.\eta \frac{\partial E_{k}(\vec{w})}{\partial \vec{w}}\right|_{\text {wn wow }}
$$

or

$$
\Delta \vec{w}_{\text {now }}=\vec{w}_{\text {next }}-\vec{w}_{\text {now }}=-\left.\eta \frac{\partial E_{T r}(\vec{w})}{\partial \vec{w}}\right|_{w=w_{\text {now }}}
$$

where, the learning rate $\eta$ controls the step size of weight update. Equation (3.13) is called the update sample-by-sample, in which the weights are updated after each training sample is presented to the network while Equation (3.14) is called batch mode update, in which the weights are updated after all training samples are presented to the network.

The basic back propagation algorithm suffers from slower convergence and possible weight oscillation [17] which may cause the change of $\frac{\partial E_{T_{r}}}{\partial \vec{w}}$ between samples. In order to solve this situation, $\eta$ is kept small and a momentum term is added. The addition of a momentum term to the Equations (3.13) and (3.14), as proposed by [29], results in a significant improvement to the basic back propagation by reducing the weight oscillation. The updated formulae with the momentum are shown in Equations (3.15) and (3.16).

$$
\Delta \vec{w}_{\text {now }}=-\left.\eta \frac{\partial E_{k}(\vec{w})}{\partial \vec{w}}\right|_{w=w_{\text {now }}}+\mu \Delta \vec{w}_{\text {old }}=-\left.\eta \frac{\partial E_{k}(\vec{w})}{\partial \vec{w}}\right|_{w=w_{\text {now }}}+\mu\left(\vec{w}_{\text {now }}-\vec{w}_{\text {old }}\right)
$$




$$
\Delta \vec{w}_{\text {now }}=-\left.\eta \frac{\partial E_{T_{r}}(\vec{w})}{\partial \vec{w}}\right|_{w_{\text {now }}}+\mu \Delta \vec{w}_{\text {old }}=-\left.\eta \frac{\partial E_{T_{r}}(\vec{w})}{\partial \vec{w}}\right|_{w=w_{\text {now }}}+\mu\left(\vec{w}_{\text {now }}-\vec{w}_{\text {old }}\right)
$$

where, $\mu$ is the momentum factor that controls the influence of the previous weight update direction on the current weight update, and $\vec{w}_{\text {old }}$ is the previous value of $\vec{w}$. This technique is also known as the generalized delta-rule [18].

The efficiency of training by back propagation can be improved by using adaptation schemes that allow the learning rate and the momentum factor to be adaptive during learning, such as, adaptation according to training errors [28].

\subsubsection{Conjugate Gradient}

Conjugate Gradient methods are very effective for training large neural networks since they scale well with the neural network size. These methods are originally derived from quadratic minimization. In this case, the minimum of the objective function $E_{T_{r}}$ can be effectively found within $N_{w}$ iterations. Given the initial gradient $\vec{g}_{\text {initial }}=\left.\frac{\partial E_{T_{s}}}{\partial \vec{w}}\right|_{w=w_{\text {intitial }}}$, and direction vector $\vec{h}_{\text {initial }}=-\vec{g}_{\text {initial }}$, the conjugate gradient method recursively constructs two vector sequences [31], 


$$
\begin{aligned}
\vec{g}_{\text {next }} & =\vec{h}_{\text {now }}+\lambda_{\text {now }} H \vec{h}_{\text {now }} \\
\vec{h}_{\text {next }} & =-\vec{g}_{\text {next }}+\gamma_{\text {now }} \vec{h}_{\text {now }} \\
\lambda_{\text {now }} & =\frac{\vec{g}_{\text {now }}^{T} \vec{g}_{\text {now }}}{\vec{h}_{\text {now }}^{T} H \vec{h}_{\text {now }}} \\
\gamma_{\text {now }} & =\frac{\vec{g}_{\text {next }}^{T} \vec{g}_{\text {next }}}{\vec{g}_{\text {now }}^{T} \vec{g}_{\text {now }}} \\
\text { or, } \left.\quad \vec{g}_{\text {next }}-\vec{g}_{\text {now }}\right)^{T} \vec{g}_{\text {next }} & \vec{g}_{\text {now }} \vec{g}_{\text {now }}
\end{aligned}
$$

where, $\vec{h}$ is the conjugate direction, $H$ is the Hessian matrix of the objective function $E_{T_{r}}$ and $\lambda, \gamma$ are two scalars. Here, Equation (3.20) is called the Fletcher-Reeves formula [32] and Equation (3.21) is called the Polak-Ribiere formula [33]. In order to avoid the Hessian matrix computation for determining the conjugate direction, one needs to proceed from $\vec{w}_{\text {now }}$, along the direction $\vec{h}_{\text {now }}$ to the local minimum of $E_{T_{r}}$ at $\vec{w}_{\text {next }}$ through line minimization, and then set $\vec{g}_{\text {next }}=\left.\frac{\partial E_{T_{r}}}{\partial \vec{w}}\right|_{w=w_{\text {next }}}$. This $\vec{g}_{\text {next }}$ can be used as the vector of Equation (3.17). In this way, Equation (3.19) is no longer needed and the descent direction runs along the conjugate direction, which can be accumulated without matrix computations. 


\subsection{Quality Evaluation of Neural Networks}

The final quality, including the generalization capability of a trained neural model is tested with an independent set of data, called test data. The accuracy of the trained neural model is measured by the average percentage error of the neural model output versus test data. Different factors [17] that indicate the quality of the neural model are discussed briefly in the subsequent discussion.

The relative error for the $j^{\text {th }}$ output of neural model on $k^{\text {th }}$ data sample is defined by,

$$
\delta_{k j}=\frac{y_{j}\left(x_{k}, \vec{w}\right)-d_{j k}}{d_{\max , j}-d_{\min , j}}, j=1, \ldots, m \text { and } k \in T_{e}
$$

where, $T_{e}$ is the index set of test data.

A quality measure based on the $p^{\text {th }}-$ norm measure is then defined as,

$$
M_{p}=\left[\sum_{k \in T_{e}} \sum_{j=1}^{m}\left|\delta_{k j}\right|^{p}\right]^{\frac{1}{p}}
$$

When $p=1$, the average test error can be directly calculated from $M_{1}$ as,

$$
\text { Average Test Error }=\frac{M_{1}}{\operatorname{Size}\left(T_{e}\right) m}=|\bar{\delta}|
$$

where, $|\vec{\delta}|$ is the mean value of absolute error $\left|\delta_{k j}\right|$ for all $k$ and $j$. 
When $p=\infty$, the $p^{t h}$-norm measure is the maximum test error, also known as worstcase error among the entire test data and all model outputs, that is,

$$
\text { Worst-case Error }=M_{\infty}=\max _{k \in T_{e}} \max _{j=1}^{m}\left|\delta_{k j}\right|
$$

The error mean $\bar{\delta}$ can be defined as,

$$
\operatorname{Mean}(\delta)=\frac{1}{\operatorname{Size}\left(T_{e}\right) m} \sum_{j=1}^{m} \sum_{k \in T_{e}} \delta_{k j}=\bar{\delta}
$$

The histogram of absolute error $|\delta|$ can be a useful graphical representation of the quality of a neural model.

The correlation between neural model and test data, called the correlation coefficient, is defined as,

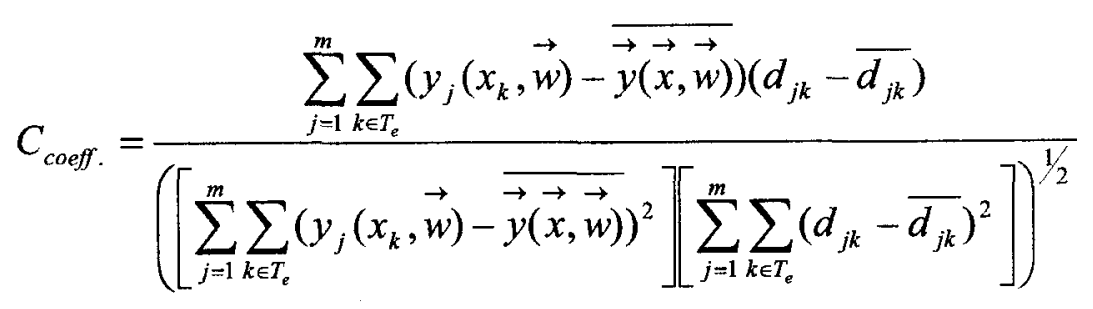

where, $\vec{y}(\vec{x}, \vec{w})$ represents the mean of the neural network outputs given by,

$$
\overrightarrow{\vec{y}(\vec{x}, \vec{w})}=\frac{1}{\operatorname{Size}\left(T_{e}\right) \cdot m} \sum_{j=1}^{m} \sum_{k \in T_{e}} y_{j}\left(x_{k}, \vec{w}\right)
$$


and $\overline{d_{j k}}$ represents the mean of the measured/simulated outputs in the test data given by,

$$
\overline{d_{j k}}=\frac{1}{\operatorname{Size}\left(T_{e}\right) \cdot m} \sum_{j=1}^{m} \sum_{k \in T_{e}} d_{j k}
$$

\subsection{Summary}

In this chapter, an overview on the neural network-based system modeling technique has been presented. Different neural network structures and training algorithms have been discussed. It appears that in order to solve difficult and diverse problems, Multi Layer Perceptrons (MLP) structures can be utilized by training them in a supervised manner with the two widely used algorithms: Back Propagation (BP) and Conjugate Gradient (CG). Consequently, these approaches have been adopted in this thesis to perform thermal analysis on electronic packaging as discussed in the subsequent chapters. 


\section{Chapter 4}

\section{Sub-model Definition}

This chapter begins with a discussion on the geometric definition of sub-models for use in hierarchical modeling. It then presents the results used for the definition of sub-model of three package models using linear Atar simulations. These simulations are performed in order to decide the number of inputs, i.e., the appropriate port configuration for neural network modeling.

\subsection{Hierarchical Modeling Using Sub-model}

In using hierarchical modeling it is necessary to build appropriate $s u b$-models that can accurately represent the thermal behavior of the original complex component-model. To create a sub-model the original detailed model of the component is used [5]. This model would accurately represent or reconstruct the physical geometry of the component very closely. A detailed model which physically models the actual device geometry can be created by using a numerical simulator, such as, Atar. This model would be a discretized version of the physical model and can be mathematically described by a large set of coupled non-linear equations. A detailed model can predict the temperatures of the 
various elements within the package (including junction, case, and leads) accurately regardless of the thermal environment in which it is placed, i.e., this model is, by definition, boundary condition independent (BCI). We wish to create or define a $s u b$ model from this detailed model.

A sub-model replaces the complex detailed component-model with a simple mathematical relationship between port temperatures and port heat-flows. To create a sub-model it is needed to extract this relationship from the detailed model. It is worthwhile to mention that the sub-models should also be able to produce all the temperatures of interest in the detailed model, such as, junction temperatures. To create a complete simulation a sub-model is linked to a detailed numerical model called basemodel as shown in Figure 4.1. This figure shows a base-model consisting of substrate and metallization, and two components. One component is a fully detailed component-model and the other is represented as a sub-model. This is an example of an Atar mixed-model.

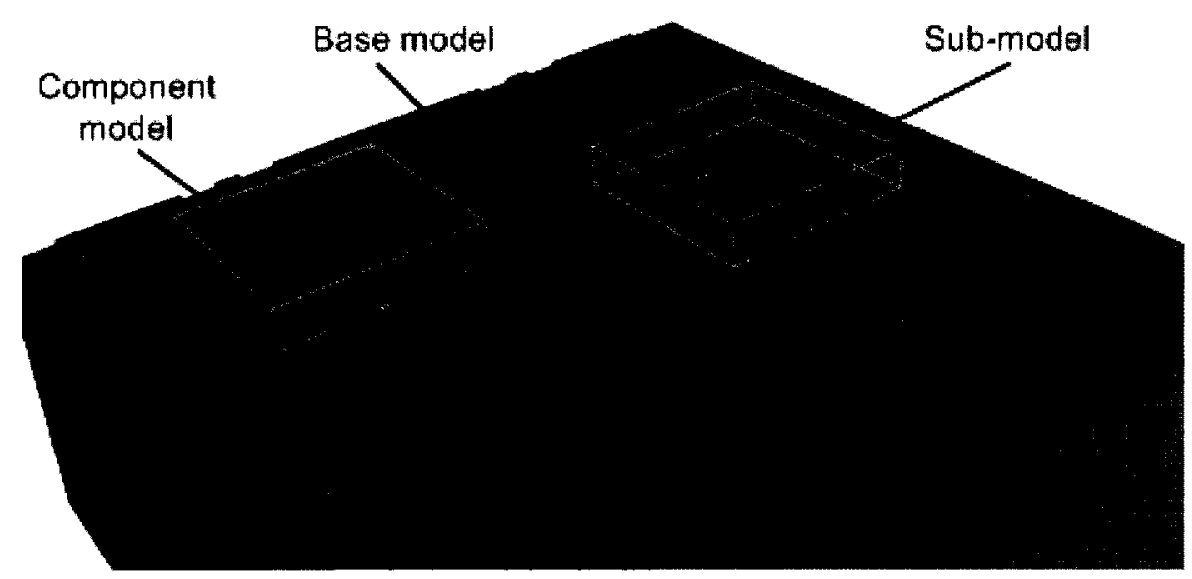

Figure 4.1: Atar Mixed-model Showing a Base-model, a Detailed Component-model and a Sub-model 
The interface or contact surfaces between the sub-model and the base-model are designated as thermal ports where the thermal ports allow the linking between the models. As a result, the sub-model can exchange heat with the base-model.

The definition of the sub-model requires the geometric specification of the ports on the detailed component-model and of the temperatures of interest. Inputs for the sub-model are therefore port temperatures $\left(T_{p}\right.$ 's), input power $(P)$ and applied boundary conditions $\left(B C\right.$ 's). Outputs are port heat-flows ( $I_{p}$ 's) and temperatures of interest $\left(T_{j}\right.$ 's). In general these relationships are non-linear and we have:

$$
\begin{aligned}
& \vec{I}_{p}=f\left(\vec{T}_{p}, \vec{P}, \overrightarrow{B C}\right) \\
& \vec{T}_{j}=g\left(\vec{T}_{p}, \vec{P}, \overrightarrow{B C}\right)
\end{aligned}
$$

These relationships [4] can be mathematically extracted efficiently for linear models leading to

$$
\begin{aligned}
& \vec{I}_{p}=G_{p} \vec{T}_{p}+\vec{F}_{p} \\
& \vec{T}_{j}=A_{p p} \vec{T}_{p}+\vec{T}_{p p}
\end{aligned}
$$

where, $G_{p} \in \mathfrak{R}^{n_{p} \times n_{p}}$ is the discretized thermal conductance matrix, $\vec{F}_{p} \in \mathfrak{R}^{n_{p} \times 1}$ is the source vector representing internal power generation and applied boundary conditions, 
$A_{p p} \in \mathfrak{R}^{n_{p} \times n_{p}}$ is the transformation matrix and $\vec{T}_{p p} \in \mathfrak{R}^{n_{p} \times 1}$ is the source temperature. For non-linear models, the relationships can be extracted from the detailed model using neural network training.

A primary concern in the definition of the sub-model is its accuracy. The accuracy of a sub-model depends on the number of ports as well as the geometric port configuration. In general, increasing the number of ports will increase the accuracy. However, as the prime consequence of the port is the imposition of constant temperature region over the port, the choice of port geometry is very important. To determine the accuracy of a given sub-model port configuration, two Atar models are built: (1) A fully detailed model of the component and base-model. (2) A mixed-model using a detailed base-model and a submodel for the component. These two models can then be compared with respect to the temperature distribution in the base and the temperatures of interest in the component.

In order to evaluate the consequences of using ports, the error is calculated for various points of interest for many port configurations of the sub-model using Equation (4.5).

$$
\text { Error }=\frac{\left|T_{\text {det }}-T_{\text {sub }}\right|}{T_{\text {det }}-300}
$$

where, $T_{\text {det }}$ is the temperature at a point of interest in the fully detailed model and $T_{\text {sub }}$ is the corresponding temperature of the mixed-model or sub-model. Previous work [34] in this area confirms that the accuracy of a sub-model increases with the increase in the 
number of ports. This is due to the fact that more information in the original model is captured as a greater number of ports are employed.

\subsection{Sub-model Definition Using Atar}

The first task in the creation of a non-linear sub-model from a component-model is an evaluation of the error inherent in various port configurations and the appropriate selection of a particular port configuration. In order to select an appropriate sub-model for neural network modeling linear simulations $\left(\right.$ with $\left.K=K_{300}\right)$ have been performed on both a detailed model and a mixed-model using a variety of linear sub-models built using Atar. The main purposes of performing these simulations discussed are:

- To evaluate the use of each sub-model for a variety of boundary conditions applied to the base-model.

- To determine the importance of the presence of ports in effecting the temperatures in the base-model and the component-model.

- To determine the minimum number of inputs (ports) that can produce an accurate sub-model with which to build a non-linear neural network model that can represent the accurate thermal behavior of the original model.

It is assumed here that for the evaluation of the port configurations using linear submodels will provide a sufficiently accurate response for determining the number of inputs 
for non-linear modeling. Linear sub-models can be generated very quickly using Atar and generate temperatures at different points of interest for a variety of BCs. In order to determine the minimum numbers of inputs required for neural network modeling, errors at different temperature points are calculated using Equation (4.5) for different port configurations of linear sub-models with respect to that of the detailed model.

It should be noted here that it is not desired to have a large number of inputs for neural network modeling because a large number of inputs would lead to a network with large input dimensions and a prohibitive amount of training. On the other hand, if the number of inputs is too few, the sub-model may not be able represent the original model accurately. Therefore, the number of inputs (and configuration of the port geometry) has to be selected accordingly. After selecting the number of inputs for neural network modeling from the linear simulations, a large number of data is generated for a variety of applied port temperatures and input power by performing non-linear simulation of the Atar component-model.

The outputs generated from Atar simulation for neural network modeling include the maximum temperature of a simple linear solution of the model, maximum temperature of a non-linear solution, port heat-flows and temperatures at different points that might be of interest. It is worthwhile to mention that increasing the number of outputs for neural network model does not lead to a more complicated model analysis. Therefore, the number of outputs might be increased when an in depth study on the model is necessary. 


\subsection{Linear Simulation Results for Package Models}

This section presents the simulation results for different IC components and packages, chosen from different technologies, as follows: (a) A Gallium Nitride ( $\mathrm{GaN}$ ) on $\mathrm{SiC}$ power package amplifier, (b) A Gallium Arsenide (GaAs) based power amplifier and (c) Ceramic-Ball-Grid-Array (CBGA) single-chip package. These models have been chosen to provide a broad range of geometries and applications to enable an evaluation of the neural network sub-modeling technique over a range of situations.

\subsubsection{GaN Based Power Package Amplifier}

It has been chosen to model non-linear $\mathrm{GaN}$ power cells consisting of $\mathrm{GaN}$ mesa structures as sub-models. These sub-models are then attached to a base-model of a $\mathrm{Si}$ substrate. Figure 4.2 shows a single cell of a GaN based power cell amplifier with six fingers on a single metal substrate. Figure 4.3 (a) shows a detailed GaN componentmodel attached to the base-model used to evaluate the sub-model and Figure 4.3 (b) shows the same model but with a sub-model attached to the base-model.

To evaluate the sub-model performance for a wide variety of heat-flow conditions both a detailed model and a sub-model of the GaN power amplifier have been simulated using Atar for six different simulation scenarios as shown in Table 4.1. For these simulations the boundary conditions on the sides of the base-model have been varied to produce different thermal conditions for the sub-model. 


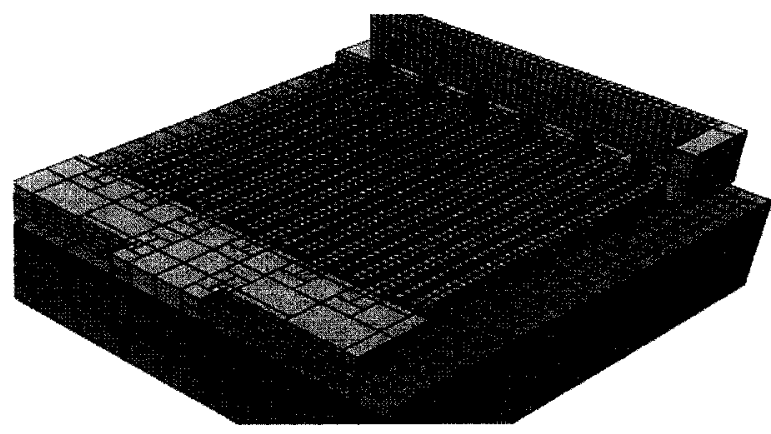

Figure 4.2: GaN Based Power Amplifier Mesa Structure
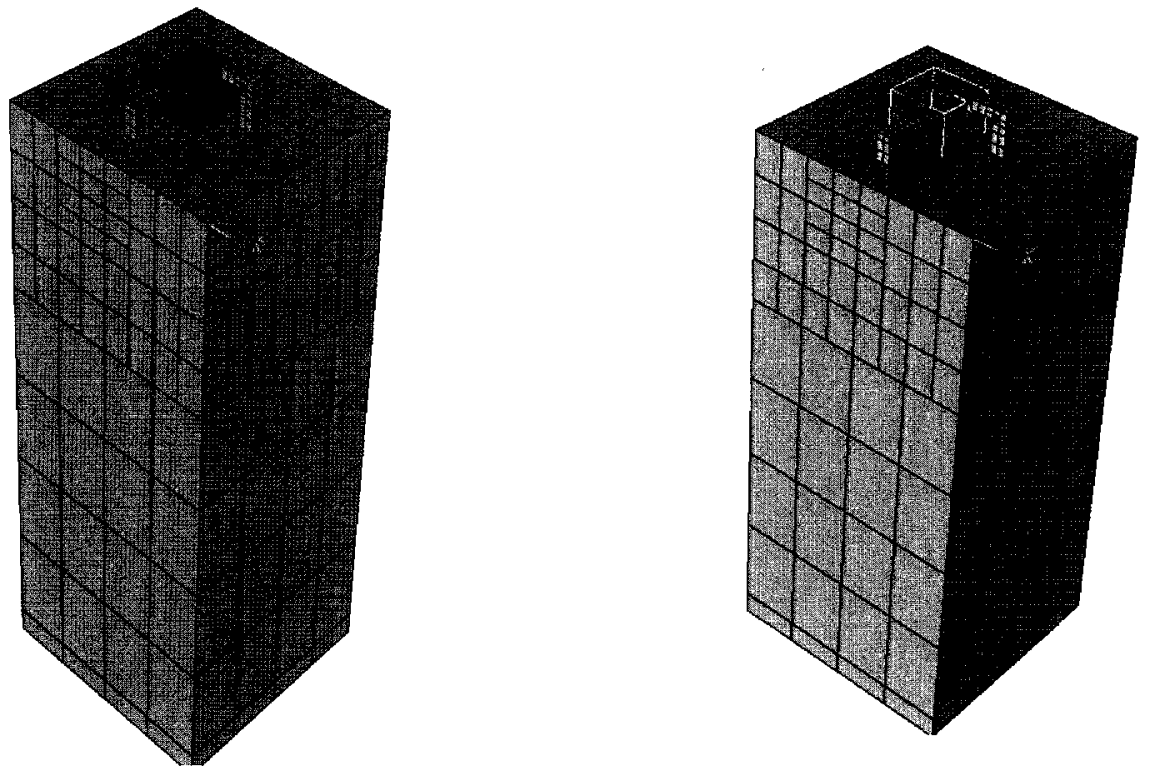

(a)

(b)

Figure 4.3: (a) GaN Component-model Attached to a Base-model (b) GaN Submodel Attached to a Base-model 
Table 4.1: Different Scenarios for Linear Simulation of GaN Model

\begin{tabular}{|c|c|c|}
\hline Case-1 & \multirow{6}{*}{$\mathrm{P}=0.002 \mathrm{~W}$} & $\mathrm{~T}=300 \mathrm{~K}$ for back, left, right, up and down-sides \\
\hline Case-2 & & $\mathrm{T}=300 \mathrm{~K}$ for back-side; no heat-flow for other sides \\
\hline Case-3 & & $\mathrm{T}=300 \mathrm{~K}$ for left $\mathrm{side} ; \quad$ no heat-flow for other sides \\
\hline Case-4 & & $T=300 \mathrm{~K}$ for right-side; no heat-flow for other sides \\
\hline Case-5 & & no heat-flow for other sides \\
\hline Case-6 & & $\mathrm{T}=300 \mathrm{~K}$ for down-side; no heat-flow for other sides \\
\hline
\end{tabular}

Basic ports of the model include the following regions: (a) The three metal connections (b) Four regions for the bottom of the GaN model as shown in Figure 4.4 (a) and Figure 4.4 (b). To determine the effect of port configuration on the performance of the submodel six different port configurations of the sub-model have been tested as follows:

- Nine Ports - Four ports on the bottom, two ports for each of the two metal contacts at the back, i.e., a total of four ports and one port for the metal contact on the side forms nine different ports.

- Seven Ports - Four ports on the bottom and three metal ports form seven different ports.

- Five Ports - Four points in the bottom form four ports while all the metal points together form another single port.

- Four Ports - Three metal points form three different ports while the bottom of the sub-model forms another single port.

- Two Ports - All the metal points together form one port and the bottom of the sub-model forms another port.

- One Port - All the points together (three points in the metal and the rest six points) form a single port. 


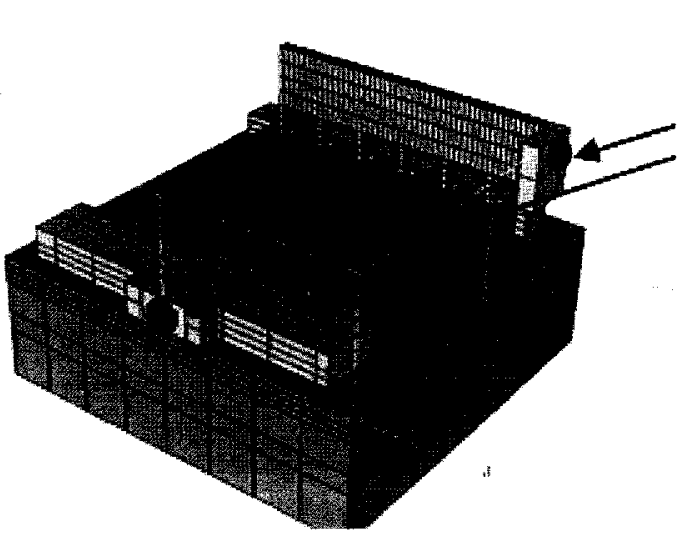

(a)

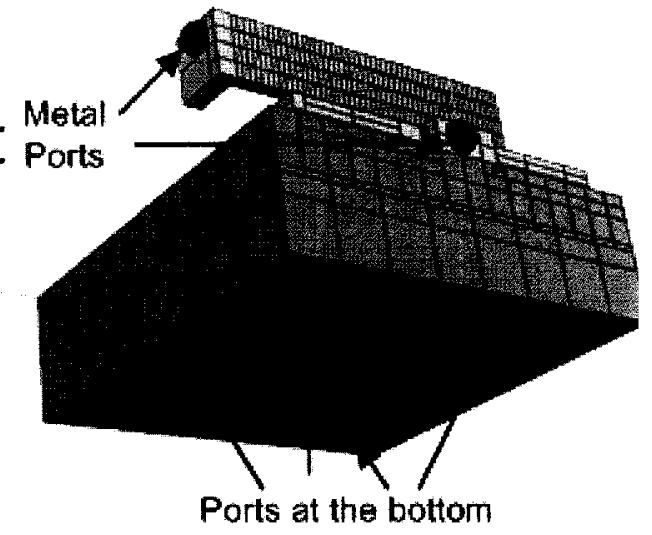

(b)

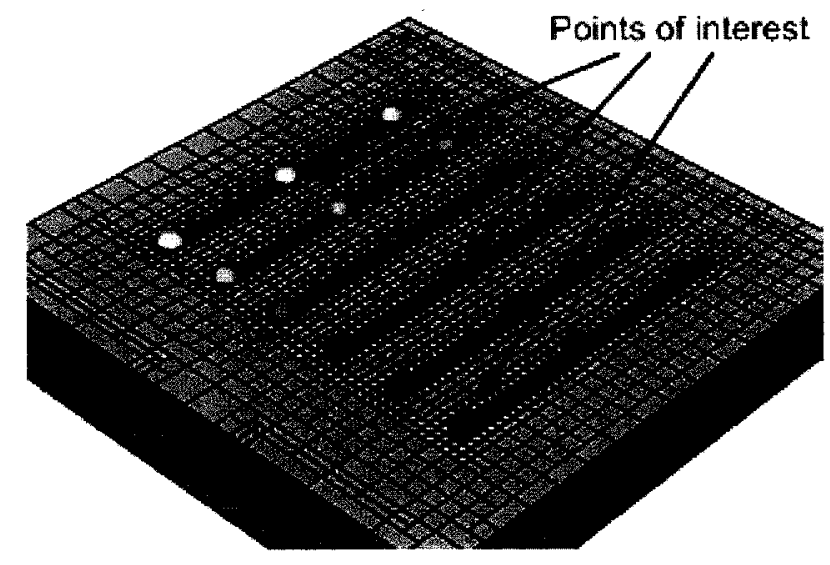

(c)

Figure 4.4: GaN Mixed-model Showing (a) Metal Ports (b) Metal Ports and Ports at the Bottom (c) Different Temperature Points (I1_1 - I3_6) of Interest 
The outputs of the linear simulation obtained from Atar for both detailed and sub-model include the temperatures at the different points that might be of interest. These points (shown in Figure 4.4) are as follows: three points in each of the six fingers, i.e., a total of eighteen points; three metal points; and four points in the bottom. The simulation results for this model for case -2 in Table 4.1 are shown in Table 4.2 and Table 4.3.

Table 4.2: The Output Temperatures (for Case - 2 in Table 4.1) of GaN Model

\begin{tabular}{|c|c|c|c|c|c|c|c|}
\hline Points & T_detail & T 9port & T 7port & T_5port & T 4port & T_2port & T_1port \\
\hline & $(\mathbf{K})$ & $\mathbf{( K )}$ & $\mathbf{( K )}$ & $\mathbf{( K )}$ & $\mathbf{( K )}$ & $(\mathbf{K})$ & (K) \\
\hline$I 1$ I & 314.54 & 314.90 & 314.86 & 314.76 & 314.85 & 314.77 & 314.77 \\
\hline$I I 2$ & 315.56 & 315.82 & 315.82 & 315.74 & 315.80 & 315.73 & 315.74 \\
\hline$I 13$ & 314.74 & 315.05 & 315.02 & 314.97 & 314.98 & 314.94 & 314.94 \\
\hline$I 14$ & 315.08 & 315.36 & 315.27 & 315.17 & 315.27 & 315.18 & 315.18 \\
\hline$I I 5$ & 316.33 & 316.49 & 316.46 & 316.38 & 316.44 & 316.37 & 316.37 \\
\hline$I 16$ & 315.31 & 315.52 & 315.44 & 315.40 & 315.41 & 315.37 & 315.37 \\
\hline$I 21$ & 315.52 & 315.52 & 315.39 & 315.28 & 315.39 & 315.29 & 315.29 \\
\hline$I 22$ & 316.83 & 316.70 & 316.64 & 316.56 & 316.63 & 316.56 & 316.56 \\
\hline$I 23$ & 315.74 & 315.68 & 315.54 & 315.50 & 315.52 & 315.48 & 315.48 \\
\hline$I 24$ & 315.72 & 315.64 & 315.50 & 315.39 & 315.52 & 315.41 & 315.40 \\
\hline$I 25$ & 316.65 & 316.49 & 316.42 & 316.35 & 316.42 & 316.35 & 316.35 \\
\hline$I 26$ & 315.75 & 315.66 & 315.53 & 315.50 & 315.52 & 315.49 & 315.48 \\
\hline$I 31$ & 315.24 & 315.49 & 315.37 & 315.27 & 315.40 & 315.30 & 315.29 \\
\hline$I 32$ & 316.44 & 316.56 & 316.48 & 316.42 & 316.50 & 316.43 & 316.43 \\
\hline$I 33$ & 315.43 & 315.63 & 315.53 & 315.52 & 315.52 & 315.50 & 315.50 \\
\hline$I 34$ & 315.00 & 315.23 & 315.13 & 315.05 & 315.17 & 315.08 & 315.07 \\
\hline$I 35$ & 315.71 & 315.84 & 315.77 & 315.71 & 315.79 & 315.72 & 315.72 \\
\hline$I 36$ & 314.97 & 315.18 & 315.10 & 315.11 & 315.10 & 315.09 & 315.09 \\
\hline$P M 1$ & 311.75 & 311.91 & 311.32 & 310.94 & 311.34 & 310.95 & 310.85 \\
\hline$P M 2$ & 311.80 & 311.71 & 311.04 & 310.77 & 311.00 & 310.76 & 310.64 \\
\hline$P M 3$ & 308.02 & 307.92 & 307.90 & 310.50 & 307.90 & 310.49 & 310.33 \\
\hline$P B 1$ & 310.54 & 311.01 & 311.07 & 310.98 & 311.07 & 311.00 & 311.01 \\
\hline$P B 2$ & 310.62 & 310.97 & 311.02 & 310.96 & 310.97 & 310.91 & 310.91 \\
\hline$P B 3$ & 310.64 & 311.04 & 311.00 & 310.93 & 311.05 & 310.98 & 310.99 \\
\hline$P B 4$ & 310.74 & 311.03 & 310.98 & 310.94 & 310.98 & 310.92 & 310.92 \\
\hline
\end{tabular}


Table 4.3: Calculated Errors for Output Temperatures (for Case - 2 in Table 4.1) of GaN Model

\begin{tabular}{|c|c|c|c|c|c|c|}
\hline Points & E_9ports & E_7ports & E_5ports & E_4ports & E_2ports & E_1port \\
\hline & & & & & & \\
\hline 111 & 0.024759 & 0.022008 & 0.015131 & 0.021320 & 0.015818 & 0.015818 \\
\hline$I 12$ & 0.016710 & 0.016710 & 0.011568 & 0.015424 & 0.010925 & 0.011568 \\
\hline 113 & 0.021031 & 0.018996 & 0.015604 & 0.016282 & 0.013569 & 0.013569 \\
\hline II 4 & 0.018568 & 0.012599 & 0.005968 & 0.012599 & 0.006631 & 0.006631 \\
\hline I1_5 & 0.009798 & 0.007961 & 0.003062 & 0.006736 & 0.002449 & 0.002449 \\
\hline$I 16$ & 0.013717 & 0.008491 & 0.005879 & 0.006532 & 0.003919 & 0.003919 \\
\hline 121 & 0.000000 & 0.008376 & 0.015464 & 0.008376 & 0.014820 & 0.014820 \\
\hline 122 & 0.007724 & 0.011289 & 0.016043 & 0.011884 & 0.016043 & 0.016043 \\
\hline 123 & 0.003812 & 0.012706 & 0.015248 & 0.013977 & 0.016518 & 0.016518 \\
\hline 124 & 0.005089 & 0.013995 & 0.020992 & 0.012723 & 0.019720 & 0.020356 \\
\hline 125 & 0.009610 & 0.013814 & 0.018018 & 0.013814 & 0.018018 & 0.018018 \\
\hline 126 & 0.005714 & 0.013968 & 0.015873 & 0.014603 & 0.016508 & 0.017143 \\
\hline 131 & 0.016404 & 0.008530 & 0.001969 & 0.010499 & 0.003937 & 0.003281 \\
\hline 1322 & 0.007299 & 0.002433 & 0.001217 & 0.003650 & 0.000608 & 0.000608 \\
\hline 133 & 0.012962 & 0.006481 & 0.005833 & 0.005833 & 0.004537 & 0.004537 \\
\hline 134 & 0.015333 & 0.008667 & 0.003333 & 0.011333 & 0.005333 & 0.004667 \\
\hline 135 & 0.008275 & 0.003819 & 0.000000 & 0.005092 & 0.000637 & 0.000637 \\
\hline 136 & 0.014028 & 0.008684 & 0.009352 & 0.008684 & 0.008016 & 0.008016 \\
\hline$P M 1$ & 0.013617 & 0.036596 & 0.068936 & 0.034894 & 0.068085 & 0.076596 \\
\hline PM2 & 0.007627 & 0.064407 & 0.087288 & 0.067797 & 0.088136 & 0.098305 \\
\hline$P M 3$ & 0.012469 & 0.014963 & 0.309227 & 0.014963 & 0.307980 & 0.288030 \\
\hline$P B 1$ & 0.044592 & 0.050285 & 0.041746 & 0.050285 & 0.043643 & 0.044592 \\
\hline$P B 2$ & 0.032957 & 0.037665 & 0.032015 & 0.032957 & 0.027307 & 0.027307 \\
\hline$P B 3$ & 0.037594 & 0.033835 & 0.027256 & 0.038534 & 0.031955 & 0.032895 \\
\hline$P B 4$ & 0.027002 & 0.022346 & 0.018622 & 0.022346 & 0.016760 & 0.016760 \\
\hline
\end{tabular}

The results for other cases mentioned in Table 4.1 are shown Tables A.1.1 to A.1.10 in Appendix A. Table 4.4 shows the average error for all the temperatures of interest for the different cases mentioned in Table 4.1. 
Table 4.4: Average Errors for Output Temperatures of GaN Model

\begin{tabular}{|c|c|c|c|c|c|c|}
\hline $\begin{array}{c}\text { Ports } \\
\text { Cases }\end{array}$ & E_9 Ports & E_7 Ports & E_5 Ports & E_4 Ports & E_2 Ports & E_1 Port \\
\hline 1 & 0.026955 & 0.032180 & 0.066311 & 0.032294 & 0.066244 & 0.065490 \\
\hline 2 & 0.015468 & 0.018385 & 0.030626 & 0.018445 & 0.030475 & 0.030523 \\
\hline 3 & 0.023606 & 0.028320 & 0.050497 & 0.028432 & 0.050477 & 0.050087 \\
\hline 4 & 0.022987 & 0.027563 & 0.058763 & 0.027710 & 0.058538 & 0.058086 \\
\hline 5 & 0.022775 & 0.027646 & 0.053027 & 0.027747 & 0.053106 & 0.052871 \\
\hline 6 & 0.024264 & 0.028299 & 0.052756 & 0.028191 & 0.052340 & 0.051994 \\
\hline Average & 0.022676 & 0.027066 & 0.051997 & 0.027137 & 0.051863 & 0.051509 \\
\hline
\end{tabular}

It has been observed that the nine-port configuration gives the best result, i.e., less error for all the cases. For five-port, two-port and one-port configurations, the errors are higher than that of the seven-port and four-port configurations. It can be concluded that the nineport, seven-port and four-port configurations give less error, but the number of ports for the nine-port and seven-port configurations are too high for neural network modeling. Considering all the port configurations, the four-port configuration has been primarily selected for neural network modeling. However, in addition, the five-port configuration has also been investigated. 


\subsubsection{GaAs Based Power Amplifier}

The second example is for a GaAs power cell. For this case a piece of substrate is defined as a sub-model. The component-model is shown in Figure 4.5 (a) and is composed of a $32 \times 32 \times 32 \mu \mathrm{m}$ piece of substrate and a four finger FET (shown in Figure 4.5 (a)). Figure 4.5 (b) shows a simple power cell of GaAs based power amplifier having metal connections placed in a simple substrate. To evaluate the sub-model performance for a wide variety of heat-flow conditions both a detailed model and a sub-model of the GaAs power amplifier have been simulated using Atar for six different simulation scenarios as shown in Table 4.5. For these simulations the boundary conditions on the sides of the base-model have been varied to produce different thermal conditions for the sub-model.

Table 4.5: Different Scenarios for Linear Simulation of GaAs Model

\begin{tabular}{|c|c|c|}
\hline Case-1 & \multirow{6}{*}{$\mathrm{P}=0.075 \mathrm{~W}$} & $\mathrm{~T}=300 \mathrm{~K}$ for back, left, right, up and down-side \\
\hline Case-2 & & $\mathrm{T}=300 \mathrm{~K}$ for back-side; Other sides free \\
\hline Case-3 & & $\mathrm{T}=300 \mathrm{~K}$ for $l e f t$-side \\
\hline Case-4 & & $\mathrm{T}=300 \mathrm{~K}$ for right-side; Other sides free \\
\hline Case-5 & & $\mathrm{T}=300 \mathrm{~K}$ for $u p$-side; \\
\hline Case-6 & & $\mathrm{T}=300 \mathrm{~K}$ for down-side; Other sides free \\
\hline
\end{tabular}

Figure 4.6 shows the GaAs sub-model with boundary condition where the dark green in the middle represents links to the ports, red color regions represents no heat-flow and light green towards the right-side of the figure represents fixed $\mathrm{BC}$. 


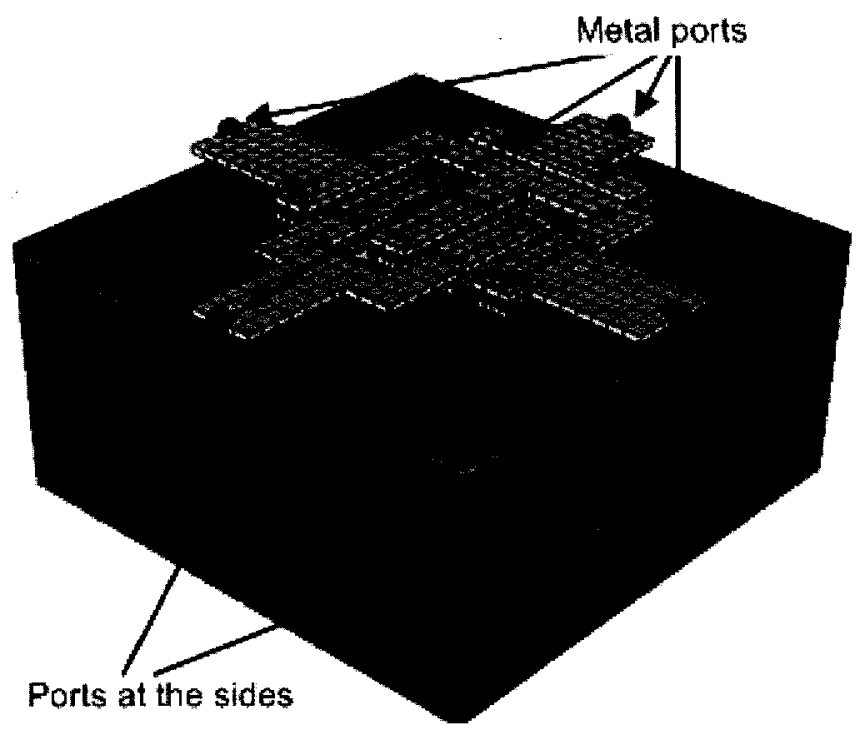

(a)

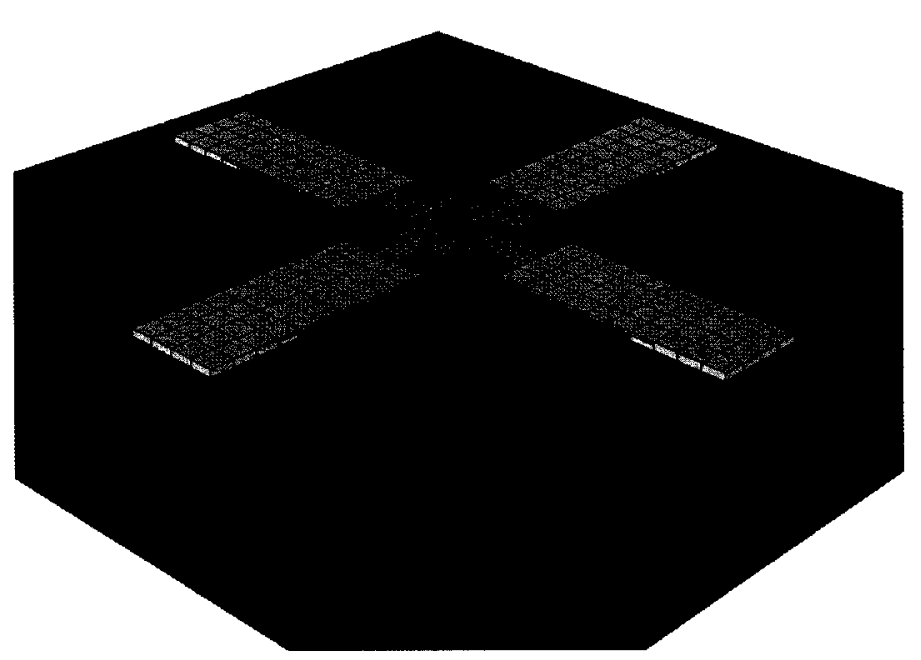

(b)

Figure 4.5: GaAs Power Amplifier (a) Component-model Showing Metal Ports and Ports at the Sides (b) Sub-model Attached to a Base-model 


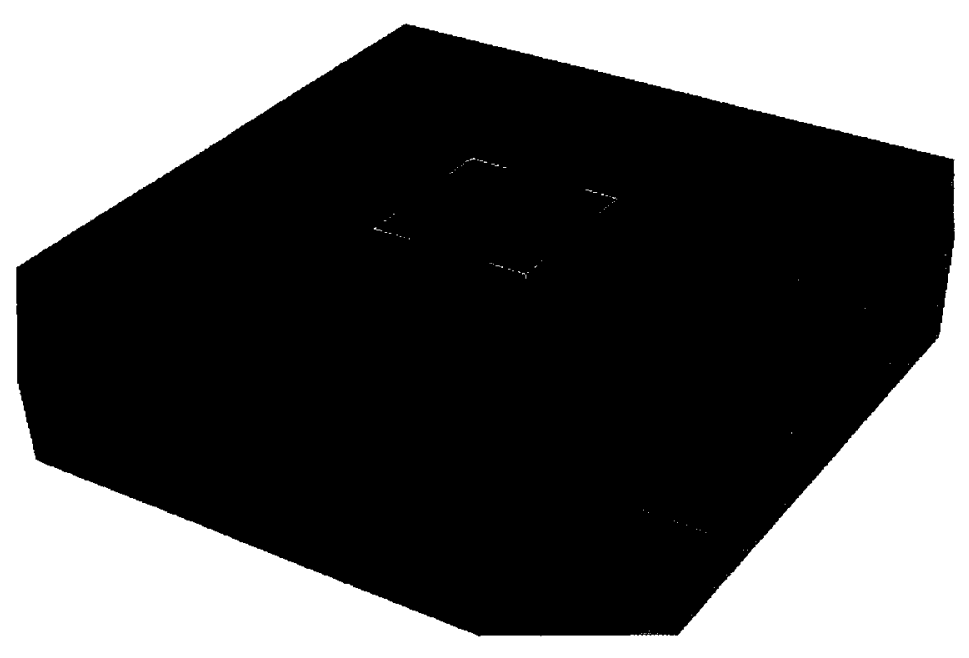

Figure 4.6: GaAs Sub-model Showing the Boundary Condition

Basic ports of this model include the following regions: (a) Four metal regions (b) Five regions for the five sides of the substrate as shown in Figure 4.5 (a). Different port configurations applied to sub-model are:

- Nine ports - Five points (PB1 - PB5) in the five different sides of the sub-model and the four metal points (PM1 - PM4) form nine different ports.

- Five ports - Four metal points form four different ports while the substrate of the sub-model forms another single port.

- Two ports - All the metal points together form one port and the substrate of the sub-model forms another single port.

- One port - All the points together (four points in the metal and the rest five points) form a single port. 
Temperatures obtained at different points of the detailed and sub-model are: three points in each of the four fingers, i.e., a total of twelve points; four metal points; and five other points in five sides involved in the port configuration of the sub-model The simulation results for this model for case -3 in Table 4.5 are shown in Table 4.6 and Table 4.7.

Table 4.6: The Output Temperatures (for Case -3 in Table 4.5) of GaAs Model

\begin{tabular}{|c|c|c|c|c|c|}
\hline Points & T detailed & T 9ports & T 5ports & T 2ports & T_1port \\
\hline & $(\mathbf{K})$ & $(\mathbf{K})$ & $(\mathbf{K})$ & $(\mathbf{K})$ & $(\mathbf{K})$ \\
\hline$I I I$ & 669.76 & 666.10 & 665.99 & 666.18 & 665.19 \\
\hline$I 12$ & 1009.55 & 1005.70 & 1005.60 & 1005.80 & 1004.70 \\
\hline$I I 3$ & 948.53 & 944.73 & 944.56 & 944.59 & 943.79 \\
\hline$I 21$ & 683.48 & 679.06 & 678.37 & 678.19 & 677.46 \\
\hline$I 22$ & 1024.62 & 1020.10 & 1019.40 & 1019.10 & 1018.40 \\
\hline$I 23$ & 963.21 & 958.63 & 957.87 & 957.54 & 957.02 \\
\hline$I 31$ & 688.85 & 683.54 & 682.33 & 681.86 & 681.10 \\
\hline$I 32$ & 1031.11 & 1025.70 & 1024.40 & 1023.80 & 1023.00 \\
\hline$I 33$ & 968.06 & 962.53 & 961.26 & 960.64 & 960.06 \\
\hline$I 41$ & 670.41 & 664.15 & 662.39 & 661.20 & 660.03 \\
\hline$I 42$ & 1008.85 & 1002.40 & 1000.60 & 999.20 & 997.90 \\
\hline$I 43$ & 950.16 & 943.71 & 941.89 & 940.69 & 939.74 \\
\hline$P M 1$ & 364.48 & 359.00 & 359.26 & 368.62 & 355.35 \\
\hline$P M 2$ & 392.75 & 384.63 & 382.58 & 369.23 & 355.99 \\
\hline$P M 3$ & 389.28 & 380.31 & 379.35 & 369.90 & 356.50 \\
\hline$P M 4$ & 363.89 & 359.06 & 358.34 & 367.27 & 354.36 \\
\hline$P B 1$ & 353.72 & 354.72 & 355.34 & 355.17 & 355.73 \\
\hline$P B 2$ & 364.14 & 358.18 & 355.59 & 355.38 & 355.94 \\
\hline$P B 3$ & 357.57 & 356.29 & 355.31 & 355.13 & 355.70 \\
\hline$P B 4$ & 356.03 & 355.58 & 354.94 & 354.79 & 355.33 \\
\hline$P B 5$ & 364.62 & 361.15 & 360.36 & 360.19 & 360.65 \\
\hline & & & & & \\
\hline
\end{tabular}


Table 4.7: Calculated Errors for Output Temperatures (for Case - 3 in Table 4.5) of GaAs Model

\begin{tabular}{|c|c|c|c|c|}
\hline Points & E_9ports & E_5ports & E_2ports & E_1port \\
\hline II 1 & 0.009898 & 0.010196 & 0.009682 & 0.012359 \\
\hline$I 1 \_2$ & 0.005370 & 0.005553 & 0.005356 & 0.006892 \\
\hline$I 13$ & 0.005859 & 0.006122 & 0.006075 & 0.007309 \\
\hline 121 & 0.011526 & 0.013325 & 0.013795 & 0.015698 \\
\hline 122 & 0.006252 & 0.007245 & 0.007673 & 0.008653 \\
\hline$I 23$ & 0.006906 & 0.008052 & 0.008549 & 0.009333 \\
\hline$I 3 I$ & 0.013656 & 0.016767 & 0.017976 & 0.019931 \\
\hline 132 & 0.007441 & 0.009137 & 0.010012 & 0.011065 \\
\hline 133 & 0.008278 & 0.010179 & 0.011107 & 0.011975 \\
\hline 141 & 0.016900 & 0.021652 & 0.024864 & 0.028023 \\
\hline 1422 & 0.009043 & 0.011596 & 0.013614 & 0.015448 \\
\hline 143 & 0.009921 & 0.012720 & 0.014566 & 0.016027 \\
\hline$P M 1$ & 0.084988 & 0.080955 & 0.064206 & 0.141594 \\
\hline$P M 2$ & 0.087547 & 0.109650 & 0.253585 & 0.396334 \\
\hline$P M 3$ & 0.100470 & 0.111223 & 0.217070 & 0.367159 \\
\hline PM4 & 0.075599 & 0.086868 & 0.052903 & 0.149163 \\
\hline$P B 1$ & 0.018615 & 0.030156 & 0.026992 & 0.037416 \\
\hline$P B 2$ & 0.092922 & 0.133302 & 0.136576 & 0.127845 \\
\hline$P B 3$ & 0.022234 & 0.039257 & 0.042383 & 0.032482 \\
\hline$P B 4$ & 0.008031 & 0.019454 & 0.022131 & 0.012493 \\
\hline$P B 5$ & 0.053699 & 0.065924 & 0.068555 & 0.061436 \\
\hline
\end{tabular}

The results for other cases mentioned in Table 4.5 are shown in Tables A.2.1 to A.2.10 in Appendix A. Table 4.8 shows the average error for all the temperatures of interest for the different cases mentioned in Table 4.5.

Table 4.8: Average Errors for Output Temperatures of GaAs Model

\begin{tabular}{|c|c|c|c|c|}
\hline $\begin{array}{c}\text { Ports } \\
\text { Cases }\end{array}$ & E_9 Ports & E_5 Ports & E_2 Ports & E_1 Port \\
\hline 1 & 0.040888 & 0.041829 & 0.067634 & 0.122851 \\
\hline 2 & 0.038349 & 0.039401 & 0.063944 & 0.117558 \\
\hline 3 & 0.031198 & 0.038540 & 0.048937 & 0.070887 \\
\hline 4 & 0.035363 & 0.042642 & 0.048987 & 0.076093 \\
\hline 5 & 0.038159 & 0.046619 & 0.073872 & 0.083669 \\
\hline 6 & 0.030938 & 0.037814 & 0.037099 & 0.069895 \\
\hline Average & 0.035816 & 0.041141 & 0.056745 & 0.090159 \\
\hline
\end{tabular}




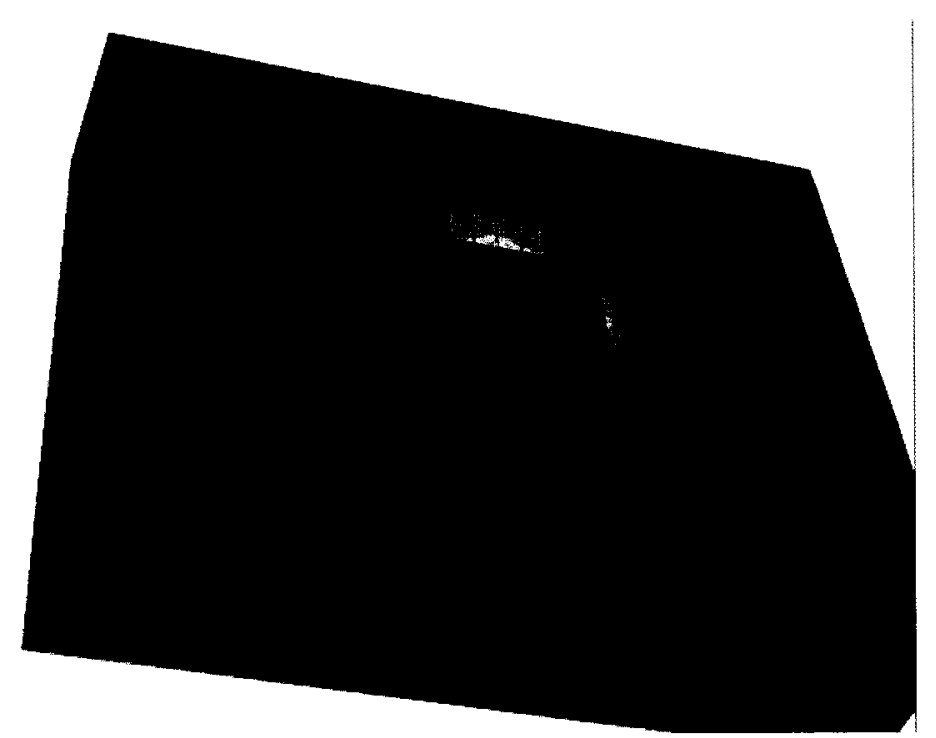

Figure 4.7: Temperature Contour for the Sub-model of GaAs Model (Fixed BC in the Front-side of the Figure)

Figure 4.7 shows the temperature contour for the mixed-model. It has been observed from Table 4.8 that the error becomes higher with the decrease in number of ports. It has also been observed that the nine-port configuration always gives better result, i.e., less error for all temperatures and with different boundary conditions. For one-port and twoport configurations, the errors are higher as compared to that of five-port configuration. It can be concluded that the nine-port configuration gives lees error but the number of ports for the nine-port configuration are too high for neural network modeling. Considering all the port configurations, the five-port configuration has been selected as the appropriate choice for neural network modeling. 


\subsubsection{Package Model}

The final example is a BGA package for the component model mounted on a PCB substrate. An additional input to the component-model is the heat-flow off the top characterized by $\alpha$. Figure 4.8 shows a single package with a simple substrate. The package model has 64 lead bumps which are located at the back-side of the model. Six different conditions for which simulations have been performed on sub-model as well as detailed model of package model are shown in Table 4.9.

Table 4.9: Different Scenarios for Linear Simulation of Package Model

\begin{tabular}{|c|c|c|c|}
\hline Case-1 & \multirow{6}{*}{$\mathrm{P}=0.5 \mathrm{~W}$} & \multirow{2}{*}{$\begin{array}{l}\alpha \text {-top }=0 \\
W / \mu \mathrm{m}^{2} \mathrm{~K}\end{array}$} & $\mathrm{~T}=300 \mathrm{~K}$ for right -side; no heat-flow for back-side \\
\hline Case-2 & & & $\mathrm{T}=300 \mathrm{~K}$ for back-side; no heat-flow for right-side \\
\hline Case-3 & & \multirow{2}{*}{$\begin{array}{c}\alpha \text {-top }=10^{-3} \\
\mathrm{~W} / \mu \mathrm{m}^{2} \mathrm{~K}\end{array}$} & $\mathrm{~T}=300 \mathrm{~K}$ for $r i g h t$-side; no heat-flow for back-side; \\
\hline Case-4 & & & $\mathrm{T}=300 \mathrm{~K}$ for $b a c k$-side; no heat-flow for right-side; \\
\hline Case-5 & & \multirow{2}{*}{$\begin{array}{c}\alpha \text {-top }=10^{-5} \\
W / \mu \mathrm{m}^{2} \mathrm{~K}\end{array}$} & $\mathrm{~T}=300 \mathrm{~K}$ for right -side; no heat-flow for $b a c k$-side; \\
\hline Case-6 & & & or back-side; no heat-flow for right-side; \\
\hline
\end{tabular}

Basic port configurations of the package model include three regions having three rings:

(a) Outer ring (b) Middle ring (c) Inner ring. The Different port configurations used for the sub-model are as follows:

- Three Ports - Three rings; where the single point in the centre of the inner ring of the sub-model forms one port, the eight points of the middle ring form the second port and the sixteen points of the outer ring of the sub-model form the third port. Figure 4.9 (a) shows the top view of the package model with three ports. 
- Two Ports - Two rings; where the single point in the centre of the inner ring form one port and the rest twenty four points (eight points of the middle ring and sixteen points of the outer ring) form another port.

- One Port - All the twenty five points together form a single port.

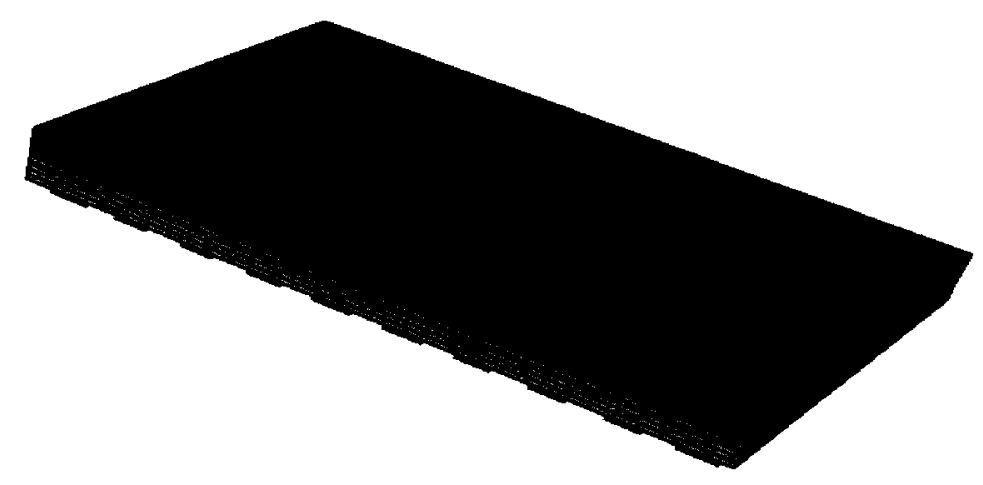

(a)

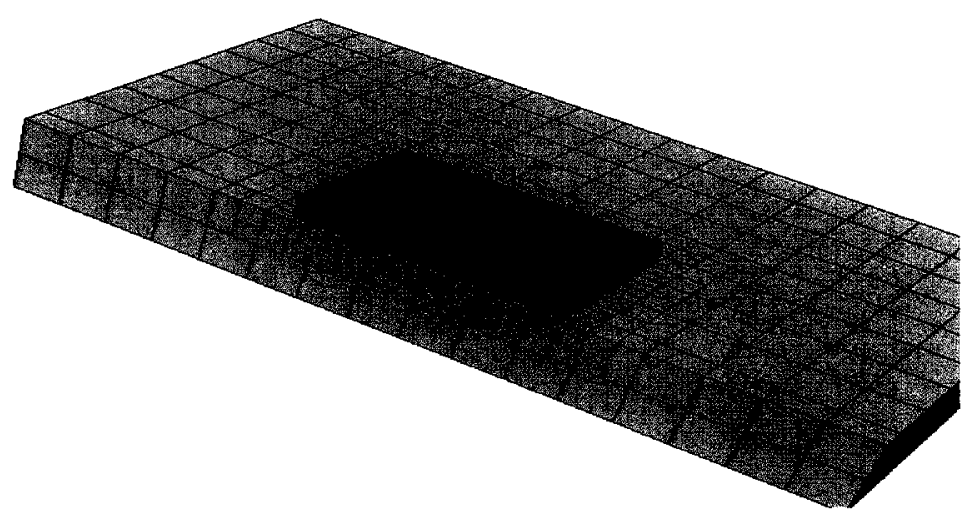

(b)

Figure 4.8: 1/2 View of Package Model (a) Sub-model Attached to Base-model (b) Component-model Attached to Base-model 


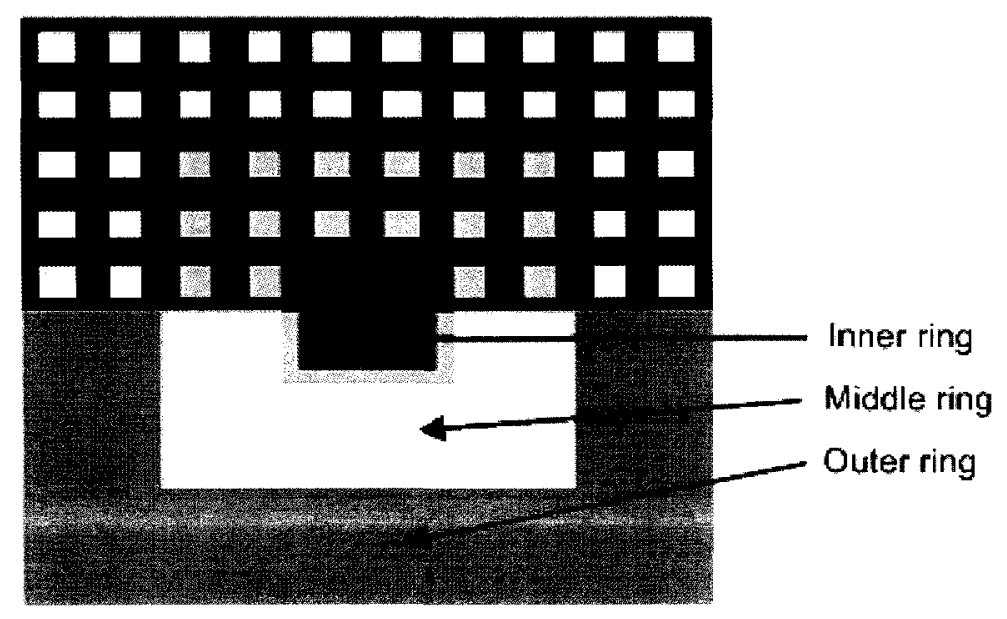

(a)

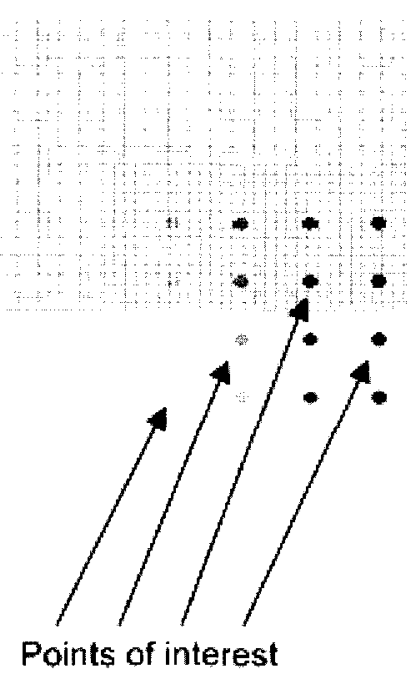

(b)

Figure 4.9: Top View of Package Model Showing (a) Three Ports (b) Different Points (P1 - P16) of Interest 
The outputs generated for this model are the temperatures in the twenty five points involved in the port configurations and the temperatures at sixteen other points (shown in Figure 4.9 (b)) of interest of the model. Errors have been calculated for all the above mentioned temperatures of the sub-model for different port configurations with respect to the corresponding temperatures of the detailed model. The simulation results for this model for case -5 in Table 4.9 are shown in Table 4.10 .

Table 4.10: Output Temperatures and Corresponding Errors (for Case -5 in Table 4.9) of Package Model

\begin{tabular}{|c|c|c|c|c|c|c|c|}
\hline Point & $T_{\text {detail }}$ & T_3ports & E_3ports & T_2ports & E_2ports & T_1port & E_1port \\
\hline & (K) & (K) & & (K) & & (K) & \\
\hline S1 & 301.82 & 301.86 & 0.021978 & 301.98 & 0.087912 & 302.04 & 0.120879 \\
\hline$S 2$ & 302.02 & 301.95 & 0.034653 & 302.02 & 0.000000 & 302.07 & 0.024752 \\
\hline S3 & 302.11 & 301.98 & 0.061611 & 302.03 & 0.037915 & 302.08 & 0.014218 \\
\hline$S 4$ & 301.99 & 301.94 & 0.025126 & 302.02 & 0.015075 & 302.07 & 0.040201 \\
\hline S5 & 301.80 & 301.85 & 0.027778 & 301.97 & 0.094444 & 302.02 & 0.122222 \\
\hline$S 6$ & 301.95 & 301.91 & 0.020513 & 301.99 & 0.020513 & 302.04 & 0.046154 \\
\hline$S 7$ & 302.32 & 302.26 & 0.025862 & 302.06 & 0.112069 & 302.09 & 0.099138 \\
\hline$S 8$ & 302.54 & 302.35 & 0.074803 & 302.12 & 0.165354 & 302.09 & 0.177165 \\
\hline 59 & 302.26 & 302.23 & 0.013274 & 302.05 & 0.092920 & 302.08 & 0.079646 \\
\hline$S 10$ & 301.91 & 301.88 & 0.015707 & 301.98 & 0.036649 & 302.03 & 0.062827 \\
\hline S11 & 301.96 & 301.90 & 0.030612 & 301.97 & 0.005102 & 302.02 & 0.030612 \\
\hline$S 12$ & 302.45 & 302.30 & 0.061224 & 302.08 & 0.151020 & 302.07 & 0.155102 \\
\hline$S 13$ & 302.78 & 302.63 & 0.053957 & 302.44 & 0.122302 & 302.08 & 0.251799 \\
\hline S14 & 302.37 & 302.27 & 0.042194 & 302.06 & 0.130802 & 302.06 & 0.130802 \\
\hline$S 15$ & 301.91 & 301.87 & 0.020942 & 301.95 & 0.020942 & 302.00 & 0.047120 \\
\hline$S 16$ & 301.77 & 301.82 & 0.028249 & 301.91 & 0.079096 & 301.96 & 0.107345 \\
\hline$S 17$ & 302.09 & 302.15 & 0.028708 & 301.98 & 0.052632 & 302.02 & 0.033493 \\
\hline$S 18$ & 302.27 & 302.22 & 0.022026 & 302.02 & 0.110132 & 302.03 & 0.105727 \\
\hline$S 19$ & 302.04 & 302.13 & 0.044118 & 301.98 & 0.029412 & 302.01 & 0.014706 \\
\hline$S 20$ & 301.73 & 301.80 & 0.040462 & 301.89 & 0.092486 & 301.94 & 0.121387 \\
\hline$S 21$ & 301.49 & 301.66 & 0.114094 & 301.77 & 0.187919 & 301.82 & 0.221477 \\
\hline$S 22$ & 301.66 & 301.74 & 0.048193 & 301.83 & 0.102410 & 301.88 & 0.132530 \\
\hline$S 23$ & 301.73 & 301.76 & 0.017341 & 301.84 & 0.063584 & 301.89 & 0.092486 \\
\hline$S 24$ & 301.64 & 301.73 & 0.054878 & 301.83 & 0.115854 & 301.87 & 0.140244 \\
\hline$S 25$ & 301.46 & 301.64 & 0.123288 & 301.75 & 0.198630 & 301.80 & 0.232877 \\
\hline$P 1$ & 301.24 & 301.22 & 0.016129 & 301.19 & 0.040323 & 301.19 & 0.040323 \\
\hline$P 2$ & 302.36 & 302.34 & 0.008475 & 302.30 & 0.025424 & 302.29 & 0.029661 \\
\hline$P 3$ & 302.36 & 302.34 & 0.008475 & 302.30 & 0.025424 & 302.29 & 0.029661 \\
\hline$P 4$ & 301.24 & 301.22 & 0.016129 & 301.19 & 0.040323 & 301.19 & 0.040323 \\
\hline
\end{tabular}




\begin{tabular}{|l|l|l|l|l|l|l|l|}
\hline$P 5$ & 302.36 & 302.34 & 0.008475 & 302.30 & 0.025424 & 302.29 & 0.029661 \\
\hline$P 6$ & 306.11 & 306.08 & 0.004910 & 306.05 & 0.009820 & 306.02 & 0.014730 \\
\hline$P 7$ & 306.11 & 306.08 & 0.004910 & 306.05 & 0.009820 & 306.02 & 0.014730 \\
\hline$P 8$ & 302.36 & 302.34 & 0.008475 & 302.30 & 0.025424 & 302.29 & 0.029661 \\
\hline$P 9$ & 302.36 & 302.34 & 0.008475 & 302.30 & 0.025424 & 302.29 & 0.029661 \\
\hline$P 10$ & 306.11 & 306.08 & 0.004910 & 306.05 & 0.009820 & 306.02 & 0.014730 \\
\hline$P 11$ & 306.11 & 306.08 & 0.004910 & 306.05 & 0.009820 & 306.02 & 0.014730 \\
\hline$P 12$ & 302.36 & 302.34 & 0.008475 & 302.30 & 0.025424 & 302.29 & 0.029661 \\
\hline$P 13$ & 301.23 & 301.22 & 0.008130 & 301.19 & 0.032520 & 301.19 & 0.032520 \\
\hline$P 14$ & 302.35 & 302.34 & 0.004255 & 302.30 & 0.021277 & 302.29 & 0.025532 \\
\hline$P 15$ & 302.35 & 302.34 & 0.004255 & 302.30 & 0.021277 & 302.29 & 0.025532 \\
\hline$P 16$ & 301.23 & 301.22 & 0.008130 & 301.19 & 0.032520 & 301.19 & 0.032520 \\
\hline
\end{tabular}

The results for other cases mentioned in Table 4.9 are shown in Table A.3.1 to Table A.3.5 in Appendix A. It can be observed from Table 4.10 and also from Table A.3.1 to Table A.3.5 that the errors become higher with the decrease in number of ports. Table 4.11 shows the average error for all the temperatures of interest for the different cases mentioned in Table 4.9.

Table 4.11: Average Errors for Output Temperatures of Package Model

\begin{tabular}{|c|c|c|c|}
\hline Ports & E_3 Ports & E_2 Ports & E_1 Port \\
\hline Cases & 0.023581 & 0.031520 & 0.034833 \\
\hline$I$ & 0.021768 & 0.051963 & 0.063044 \\
\hline 2 & 0.029430 & 0.061528 & 0.075435 \\
\hline 3 & 0.054150 & 0.137610 & 0.165352 \\
\hline 4 & 0.028759 & 0.061103 & 0.074111 \\
\hline 5 & 0.053851 & 0.135705 & 0.163926 \\
\hline 6 & 0.035257 & 0.079905 & 0.096117 \\
\hline Average & & & \\
\hline
\end{tabular}

It has been observed from Table 4.11 that the three-port configuration always provides better results for all temperatures and with different boundary conditions as compared to that of one-port and two-port configurations. Consequently, the three-port configuration has been selected as the appropriate choice for neural network modeling. 


\subsection{Summary}

It has been observed that the average errors of temperatures for different port configurations for all the three models are within satisfactory limit (For GaN, GaAs and Package model the average errors are $2 \%-5 \%, 4 \%-9 \%$ and $3 \%-10 \%$ respectively). Accordingly, the best port configuration of sub-model selected for neural network modeling for GaN, GaAs and Package model have been four-port, five-port and threeport configuration respectively. After deciding the best port configuration for each model, i.e., the inputs for neural network modeling, non-linear simulation have been performed on these three models and subsequently, a large number of data have been generated. These data have been used to train the neural model. The next chapter presents the results obtained from neural network modeling. 


\section{Chapter 5}

\section{Creation and Training of Neural Network}

\section{Modeling}

In this chapter, neural network models with three-layer and four-layer perceptrons are developed with both back propagation and conjugate gradient training method using the tool called Neuromodeler for each of the three models mentioned in Chapter 4 . Afterwards, the qualities of these models are evaluated by testing them with a set of testing data.

\subsection{Neural Network Modeling}

The primary steps to develop a neural network model are to identify the inputs and outputs of the problem to be modeled and to generate data for training and testing the model. In this thesis, the inputs for the neural network model have been decided from Atar simulations described in Section 4.2 and Section 4.3. The inputs of the neural model have been chosen as the port temperatures and power while the outputs have been chosen as the maximum temperature of a simple linear solution of the model, maximum temperature of a non-linear solution, port heat-flows for each of the input ports and 
temperatures at different points that might be of interest as shown in Figure 5.1. As indicated in Chapter 2, the modeling tool, Neuromodeler has been used to build the model. The main steps of the neural network modeling have been:

- To generate a large set of training data in order to build the neural network model for each of the three models so that the neural model could represent the original problem behavior accurately.

- To train three-layer perceptrons as well as four-layer perceptrons networks with both back propagation and conjugate gradient training methods.

- To evaluate the quality of the trained model by testing it with a different set of data for each model.

- To evaluate the performance of the two training algorithms on each model by comparing the training and testing error.

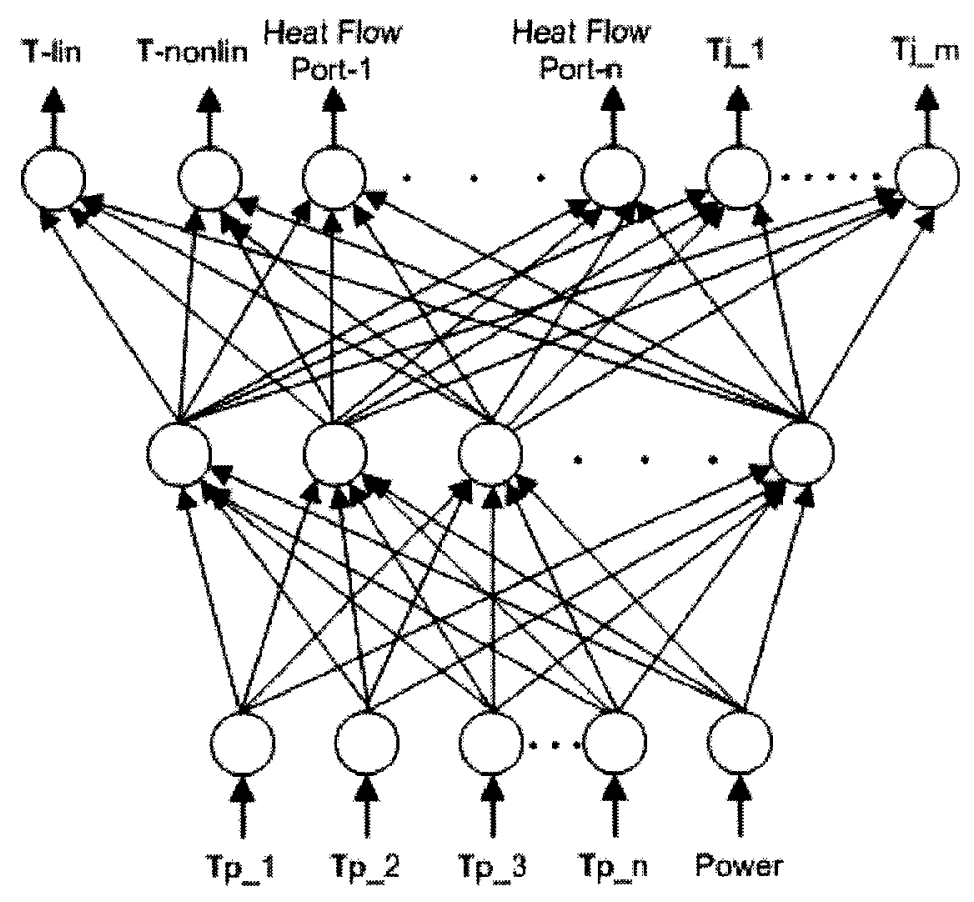

Figure 5.1: Inputs and Outputs of the Developed Neural Network Model 
The training and testing results for neural network-based modeling for all the three models mentioned earlier is presented in the subsequent sections.

\subsection{GaN Based Power Package Amplifier}

It was decided from Atar simulation mentioned in Chapter 4 that the four-port configuration would be the best choice for neural network modeling for $\mathrm{GaN}$ based power package amplifier model. In addition, the five-port configuration is to be investigated. Consequently, the inputs of this model for neural network modeling have been selected as the different port temperatures (Bot, $M 1, M 2$ and $M 3$, i.e., one port at the bottom and three metal ports for four-port configuration and Port1-Port5, i.e., four ports at the bottom and one metal port for five-port configuration) and the power. The port temperatures have been varied from $300-340 \mathrm{~K}$ (with an increase of $10 \mathrm{~K}$ ) while the power has been varied from $0.02-0 \mathrm{~W}$ (with a decrease of $0.005 \mathrm{~W}$ ). The outputs of this model include the maximum temperature (T-linear) of a simple linear solution of the model, maximum temperature ( $T$-nonlinear) of a non-linear solution, port heat-flows $(T$ bot and TM1-TM3 for four-port configuration and TP1-TP5 for five-port configuration) for each of the input ports and temperatures at 37 different points that might be of interest. A total of 3125 and 15625 training data (where each data represents a set of all the inputs and outputs) have been generated for four-port and five-port configuration respectively by performing a non-linear simulation using Atar. Afterwards, a separate set of randomly generated data (approximately 1500) has been used to evaluate the performance of the trained model. 


\subsubsection{Training of GaN Model with Four-Port Configuration}

The GaN model with four-port configuration has been trained in four ways as follows,

1) Three-layer perceptrons network with back propagation training method.

2) Three-layer perceptrons network with conjugate gradient training method.

3) Four-layer perceptrons network with back propagation training method.

4) Four-layer perceptrons network with conjugate gradient training method.

1) Three-layer with Back Propagation: The number of hidden neurons in the hidden layer of the three-layer perceptrons network has been chosen (automatically chosen by Neuromodeler) as 20 . The initial conditions (automatically chosen by Neuromodeler) of the training method have been: maximum number of epochs: 100 , learning rate: 0.001 and momentum factor: 0 . With these initial conditions, the final training error obtained has been 0.006987657 at 100 epochs (the first training has been performed for 100 epochs where the number of epochs was automatically set by Neuromodeler). It has been observed that as the network was trained further, the final training error decreased with the increase in epochs. At the end of 1000 epochs (after training the network for 10 times, the number of epochs reached 1000) the final training error has been obtained as 0.005296763 . Figure 5.2 shows the training error against the first 500 epochs. As mentioned in Chapter 3, the training error is defined as, 


$$
E_{T_{r}}(\vec{w})=\left[\frac{1}{\operatorname{size}\left(T_{r}\right) \cdot m} \sum_{k \in T_{r}} \sum_{j=1}^{m}\left|\frac{y_{j}\left(x_{k}, \vec{w}\right)-d_{j k}}{d_{\max , j}-d_{\min , j}}\right|^{p}\right.
$$

where $y_{j}\left(x_{k}, \vec{w}\right)$ is the $j^{\text {th }}$ output of the neural network for input sample $x_{k}, d_{k}$ is the measured/simulated output for $\vec{y}$ for the input $x_{k}, T_{r}$ is the index set of training data, $k \in T_{r}, m$ is the dimension of the output $\vec{y}, d_{j k}$ is the $j^{\text {th }}$ element of $d_{k}, p$ represents least $p^{\text {th }}$ optimization, $d_{\max , j}$ and $d_{\min , j}$ are the maximum and minimum values of the $j^{\text {th }}$ element of all vectors of $d_{k}$.

2) Three-layer with Conjugate Gradient: The number of hidden neurons in the hidden layer of the three-layer perceptrons network has been chosen (automatically chosen by Neuromodeler) as 20 . The initial conditions (automatically chosen by Neuromodeler) of the training method have been: maximum number of epochs: 200, function error tolerance: $1 \mathrm{e}^{-4}$ and model parameter tolerance: $1 \mathrm{e}^{-4}$. With these initial conditions, the final training error obtained has been 0.00457244 at 200 epochs (the first training has been performed for 200 epochs where the number of epochs was automatically set by Neuromodeler). It has been observed that as the network was trained further, the final training error decreased with the increase in epochs. At the end of 412 epochs (after training the network for 10 times, the number of epochs reached 412) the final training error has been obtained as 0.003389 . Figure 5.2 shows the training error against the first 412 epochs. 
3) Four-layer with Back Propagation: The number of hidden neurons in the $1^{\text {st }}$ and $2^{\text {nd }}$ hidden layer of the four-layer perceptrons network have been chosen (automatically chosen by Neuromodeler) as 5 and 3 respectively. The initial conditions (automatically chosen by Neuromodeler) of the training method have been: maximum number of epochs: 200, learning rate: 0.001 and momentum factor: 0 . With these initial conditions, the final training error obtained has been 0.0270021 at 200 epochs (the first training has been performed for 200 epochs where the number of epochs was automatically set by Neuromodeler). It has been observed that as the network was trained further, the final training error decreased with the increase in epochs. At the end of 1200 epochs (after training the network for 10 times, the number of epochs reached 1200) the final training error has been obtained as 0.0264888 . Figure 5.2 shows the training error against the first 500 epochs.

4) Four-layer with Conjugate Gradient: The number of hidden neurons in the $1^{\text {st }}$ and $2^{\text {nd }}$ hidden layer of the four-layer perceptrons network have been chosen (automatically chosen by Neuromodeler) as 5 and 3 respectively. The initial conditions (automatically chosen by Neuromodeler) of the training method have been: maximum number of epochs: 200 , function error tolerance: $1 \mathrm{e}^{-4}$ and model parameter tolerance: $1 \mathrm{e}^{-4}$. With these initial conditions, the final training errors obtained have been 0.0409873 and 0.0287286 at 110 (the first training has been performed for 110 epochs where the number of epochs was automatically set by Neuromodeler) and 240 epochs (after training the network for 10 times, the number of epochs reached 240 ) respectively. Figure 5.2 shows the training error against the 240 epochs. 
The training errors obtained in the above mentioned ways are summarized in Table 5.1.

As mentioned earlier, the number of hidden neurons and the initial conditions of the trainings have been automatically selected by the Neuromodeler.

Table 5.1: Training Errors of GaN Model with Four-Port Configuration

\begin{tabular}{|c|c|c|c|c|}
\hline $\begin{array}{c}\text { Layer/ } \\
\text { Training }\end{array}$ & $\begin{array}{c}\text { Hidden } \\
\text { Neurons }\end{array}$ & Initial Condition & $\begin{array}{c}\text { Initial Training } \\
\text { Error (After 1 } \\
\text { Training) }\end{array}$ & $\begin{array}{c}\text { Final Training } \\
\text { Error (After } \\
\text { 10 }^{\text {th }} \text { Training) }\end{array}$ \\
\hline 3 layer BP & 20 & $\begin{array}{c}\text { max. number of epochs: } 100 \\
\text { learning rate: } 0.001 \\
\text { momentum factor: 0 }\end{array}$ & $\begin{array}{c}0.006987657 \\
\text { at } 100 \text { epochs }\end{array}$ & $\begin{array}{c}0.005296763 \\
\text { at } 1000 \text { epochs }\end{array}$ \\
\hline 3 layer CG & 20 & $\begin{array}{c}\text { max. number of epochs: } 200 \\
\text { function error tolerance: } 1 \mathrm{e}^{-4} \\
\text { model parameter tolerance: } 1 \mathrm{e}^{-4}\end{array}$ & $\begin{array}{c}0.004572440 \\
\text { at } 200 \text { epochs }\end{array}$ & $\begin{array}{c}0.003389000 \\
\text { at } 412 \text { epochs }\end{array}$ \\
\hline 4 layer BP & layer1: 5 & $\begin{array}{c}\text { max. number of epochs: } 200 \\
\text { learning rate: } 0.001 \\
\text { layer2: 3 }\end{array}$ & $\begin{array}{c}0.027002100 \\
\text { at } 200 \text { epochs }\end{array}$ & $\begin{array}{c}0.026488800 \\
\text { at } 1200 \text { epochs }\end{array}$ \\
\hline 4 layer CG & layer1: 5 & $\begin{array}{c}\text { max. number of epochs: } 200 \\
\text { function error tolerance: } 1 \mathrm{e}^{-4} \\
\text { layer2: } 3\end{array}$ & $\begin{array}{c}0.040987300 \\
\text { at } 110 \text { epochs }\end{array}$ & $\begin{array}{c}0.028728600 \\
\text { at } 240 \text { epochs }\end{array}$ \\
\hline
\end{tabular}

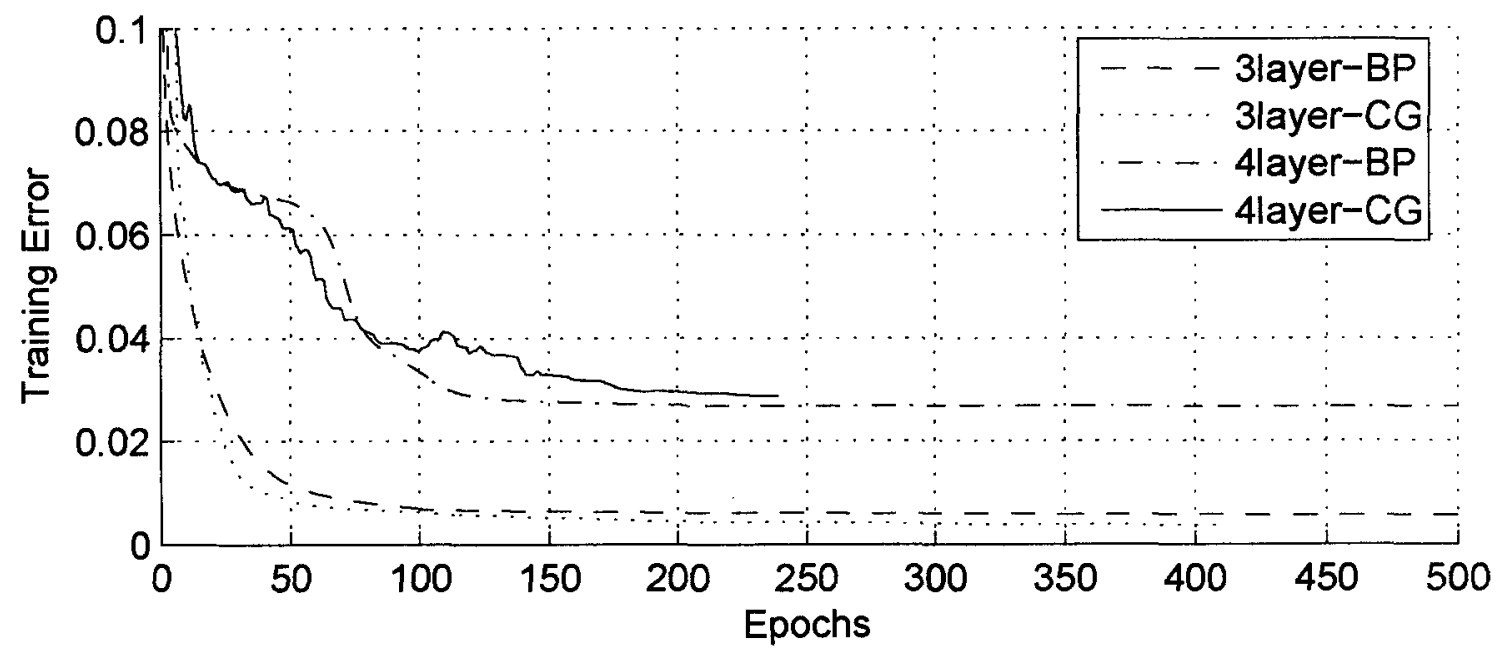

Figure 5.2: Training Error of GaN Model with Four-Port Configuration for Different Training Methods and Structures 
It has been observed from Figure 5.2 that after 200 epochs all the training curves become steady and the training errors stabilize. It has also been observed that at any epoch, the training error is less for three-layer perceptrons network than that of four-layer perceptrons network.

\subsubsection{Testing of GaN Model with Four-Port Configuration}

Once the neural network was built with the training data, the performance of the network has been tested with a separate set (unseen) of testing data. Table 5.2 shows the training and testing results for GaN model with four-port configuration.

Table 5.2: Training and Testing Results of GaN Model with Four-Port Configuration

\begin{tabular}{|c|c|c|c|c|c|}
\hline $\begin{array}{c}\text { Result } \\
\text { Training }\end{array}$ & Epochs & $\begin{array}{c}\text { Training } \\
\text { Error }\end{array}$ & $\begin{array}{c}\text { Average } \\
\text { Testing Error }\end{array}$ & $\begin{array}{c}\text { Maximum } \\
\text { Testing Error }\end{array}$ & $\begin{array}{c}\text { Correlation } \\
\text { Coefficient }\end{array}$ \\
\hline 3-layer $B P$ & 1000 & 0.005296763 & 0.504973800 & 18.039429 & 0.99999640 \\
\hline 4-layer $B P$ & 412 & 0.026488800 & 2.857109000 & 63.471737 & 0.99995610 \\
\hline 3-layer $C G$ & 1200 & 0.003733890 & 0.376287160 & 14.991369 & 0.99999803 \\
\hline 4-layer $C G$ & 240 & 0.028728600 & 3.035344100 & 66.668915 & 0.99995480 \\
\hline
\end{tabular}

It can be observed from Table 5.2 that the average testing errors and maximum testing errors are also less for models with three-layer perceptrons network than that of fourlayer perceptrons network. Also, as mentioned in Chapter 3, the correlation coefficient denotes the correlation between neural model and test data. Table 5.2 shows that the correlation coefficients are higher for models with three-layer perceptrons than that of four-layer perceptrons. Therefore, it can be concluded that the model is well trained with the conjugate gradient training as well as back propagation training method for three- 
layer perceptrons network. Figure 5.3 represents the plots for average and maximum testing error vs. epoch for $\mathrm{GaN}$ model with three-layer perceptrons.

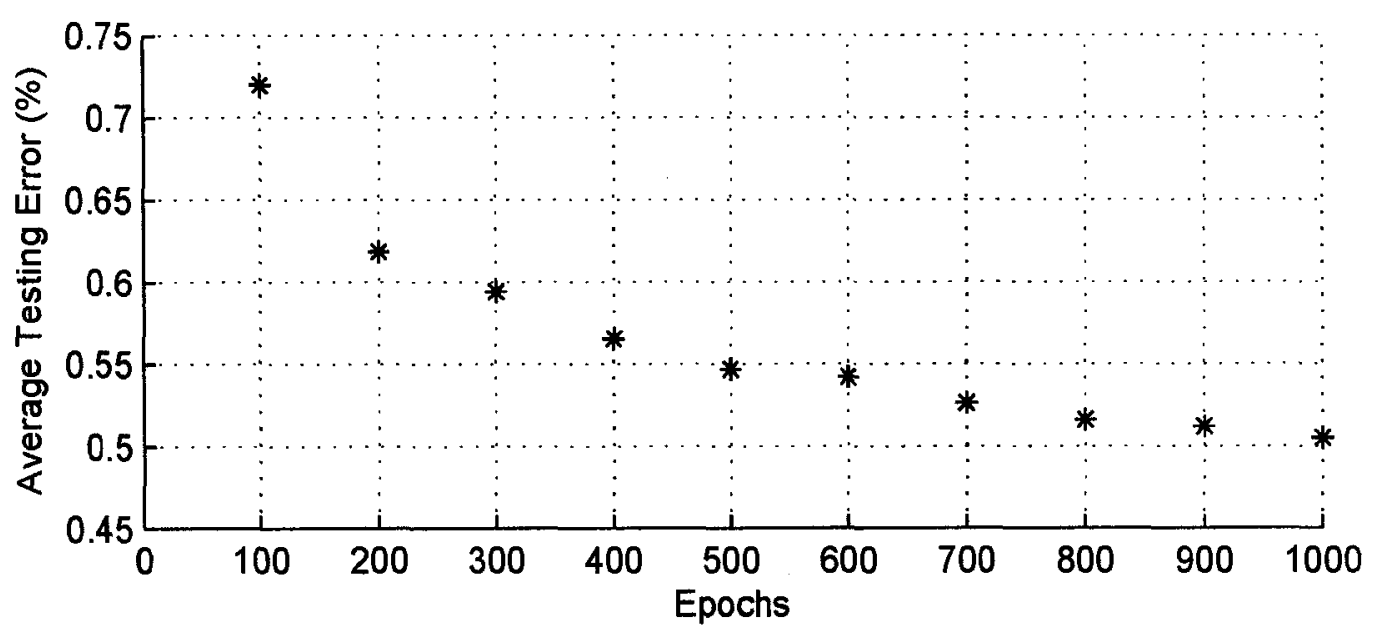

Figure 5.3 (a): Average Testing Error (\%) vs. Epoch for GaN-4 Model with ThreeLayer Perceptrons Using Back Propagation Training

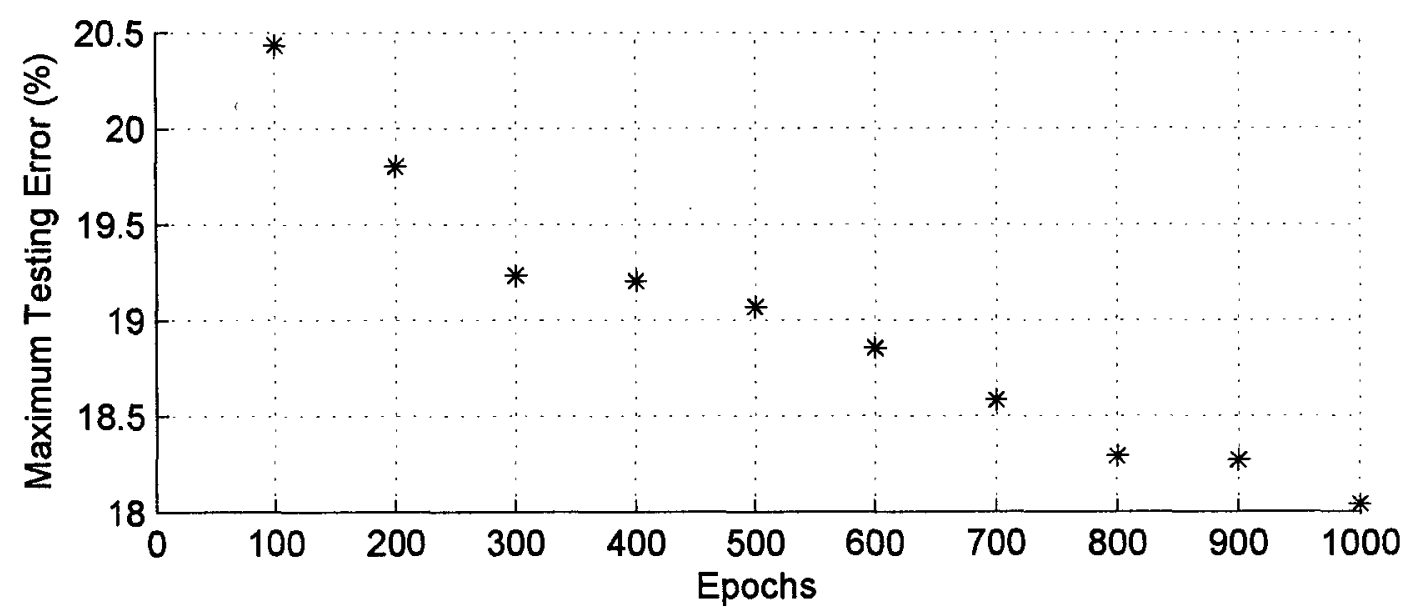

Figure 5.3 (b): Maximum Testing Error (\%) vs. Epoch for GaN-4 Model with Three-Layer Perceptrons Using Back Propagation Training 
It has been observed from Figure 5.3 that the average and maximum testing errors decrease with the increase of number of training epochs. Therefore, it can be concluded that the network is not over trained. Table 5.3 shows the average and maximum testing errors for each of the outputs for $\mathrm{GaN}$ model with three-layer perceptrons.

Table 5.3: Testing Errors of GaN Model with Three-Layer Perceptrons and FourPort Configuration

\begin{tabular}{|c|c|c|c|c|}
\hline Output & $\begin{array}{l}\text { Average Error } \\
\text { (BP3) }\end{array}$ & $\begin{array}{l}\text { Maximum Error } \\
\text { (BP3) }\end{array}$ & $\begin{array}{l}\text { Average Error } \\
\text { (CG3) }\end{array}$ & $\begin{array}{l}\text { Maximum Error } \\
\text { (CG3) }\end{array}$ \\
\hline T-linear & 2.05835440 & 18.0394380 & 1.43192550 & 12.8560190 \\
\hline T-nonlinear & 2.13536700 & 17.1612740 & 1.35329470 & 10.2419710 \\
\hline$T-b o t$ & 0.37721112 & 3.0211182 & 0.28054956 & 2.0564208 \\
\hline$T M 1$ & 0.56289625 & 2.4726080 & 0.65022000 & 8.5783380 \\
\hline$T M 2$ & 0.31648500 & 1.7962474 & 0.30199860 & 2.8126283 \\
\hline$T M 3$ & 1.15259590 & 5.3824220 & 0.36903074 & 1.6285741 \\
\hline$I 1-1$ & 0.35322627 & 3.0845199 & 0.24536760 & 2.1074777 \\
\hline $11-2$ & 0.38468698 & 3.6339493 & 0.35180047 & 2.4803240 \\
\hline$I 1-3$ & 0.30614700 & 2.5460430 & 0.24242292 & 1.9377272 \\
\hline$I 1-4$ & 0.30588250 & 2.3246558 & 0.24281265 & 2.1525698 \\
\hline$I 1-5$ & 0.33366418 & 2.0609448 & 0.28760797 & 3.4277070 \\
\hline$I 1-6$ & 0.30224845 & 1.7820654 & 0.20792589 & 1.5811069 \\
\hline$I 2-1$ & 0.34150276 & 2.4921500 & 0.32629030 & 2.8890820 \\
\hline $12-2$ & 0.31135368 & 1.7852107 & 0.24282907 & 2.4637530 \\
\hline$I 2-3$ & 0.35905495 & 2.8148563 & 0.25670642 & 2.3792675 \\
\hline$I 2-4$ & 0.32227376 & 2.7766125 & 0.23127094 & 1.7845937 \\
\hline$I 2-5$ & 0.30711270 & 2.3601599 & 0.19894058 & 1.4396828 \\
\hline$I 2-6$ & 0.28511740 & 2.3259158 & 0.19201125 & 1.4109217 \\
\hline $13-1$ & 0.36472487 & 2.7185680 & 0.21796600 & 1.5064819 \\
\hline$I 3-2$ & 0.33573255 & 2.4126415 & 0.24210289 & 2.1346107 \\
\hline $13-3$ & 0.26717153 & 1.5455226 & 0.25082460 & 1.6653341 \\
\hline $13-4$ & 0.36790776 & 3.5143754 & 0.37196410 & 2.6980913 \\
\hline $13-5$ & 0.35992740 & 2.4058177 & 0.32797354 & 3.1356711 \\
\hline$I 3-6$ & 0.45859572 & 3.6622164 & 0.20298074 & 1.6304903 \\
\hline$P M I$ & 0.69095355 & 4.3836140 & 1.12738340 & 14.991370 \\
\hline$P M 2$ & 0.76150850 & 4.2025466 & 0.41941770 & 3.5418112 \\
\hline$P M 3$ & 0.79561660 & 4.7225520 & 0.61785805 & 3.5446390 \\
\hline$P B 1$ & 0.69200030 & 4.0209620 & 0.42884672 & 3.0700994 \\
\hline$P B 2$ & 0.67983735 & 4.8049580 & 0.41028178 & 3.4015010 \\
\hline$P B 3$ & 0.65071950 & 3.8518867 & 0.39021996 & 2.2565530 \\
\hline$P B 4$ & 0.57293430 & 3.2341430 & 0.42279145 & 3.0852215 \\
\hline$P 1$ & 0.34426922 & 3.0211587 & 0.27629068 & 2.0682454 \\
\hline$P 2$ & 0.29241088 & 2.2792852 & 0.24325553 & 1.3644630 \\
\hline
\end{tabular}




\begin{tabular}{|c|c|c|c|c|}
\hline$P 3$ & 0.31247887 & 1.8916565 & 0.24532057 & 1.6781352 \\
\hline$P 4$ & 0.38857272 & 3.0215952 & 0.27944810 & 2.2310135 \\
\hline$P 5$ & 0.31763303 & 2.5104460 & 0.29194766 & 2.5090046 \\
\hline$P 6$ & 0.33388945 & 2.3694046 & 0.18980652 & 1.6118526 \\
\hline$P 7$ & 0.40847751 & 2.7208260 & 0.35844755 & 1.5627985 \\
\hline$P 8$ & 0.39311240 & 2.6294850 & 0.2994433 & 3.1214464 \\
\hline$P 9$ & 0.29907992 & 2.9477208 & 0.35012504 & 3.5816400 \\
\hline$P 10$ & 0.32220122 & 1.9993650 & 0.30104136 & 2.3180492 \\
\hline$P 11$ & 0.36420550 & 2.1221972 & 0.25071907 & 1.9728230 \\
\hline$P 12$ & 0.42473093 & 2.6068401 & 0.25088778 & 1.6395134 \\
\hline
\end{tabular}

It can be observed from Table 5.3 that the average errors and the maximum errors are within acceptable limit (average error $\leq 5 \%$ and the maximum errors $\leq 15 \%$ ) with the exceptions that the maximum errors are high for $T$-linear and $T$-nonlinear with back propagation training. These high errors have occurred only for very few samples (maximum 3-4) as compared to the total number of 1500 samples as shown in Figure 5.4 (a) and Figure 5.4 (b) for the outputs T-linear and T-nonlinear respectively. Figure 5.5 (a) and Figure 5.5 (b) show the test plots for T-linear vs. sample number and T-nonlinear vs. sample number with back propagation and conjugate gradient training method respectively for three-layer perceptrons network. 


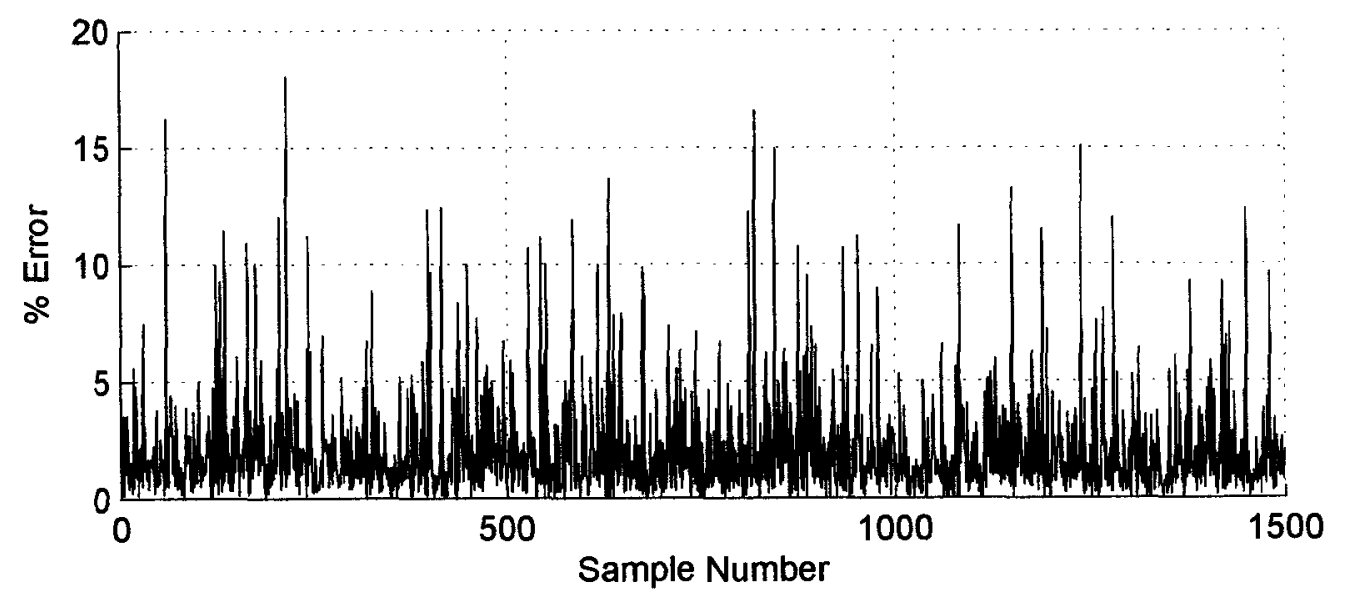

Figure 5.4 (a): Percentage Error of GaN-4 Neural Model with Three-Layer Perceptrons for T-linear Using Back Propagation Training

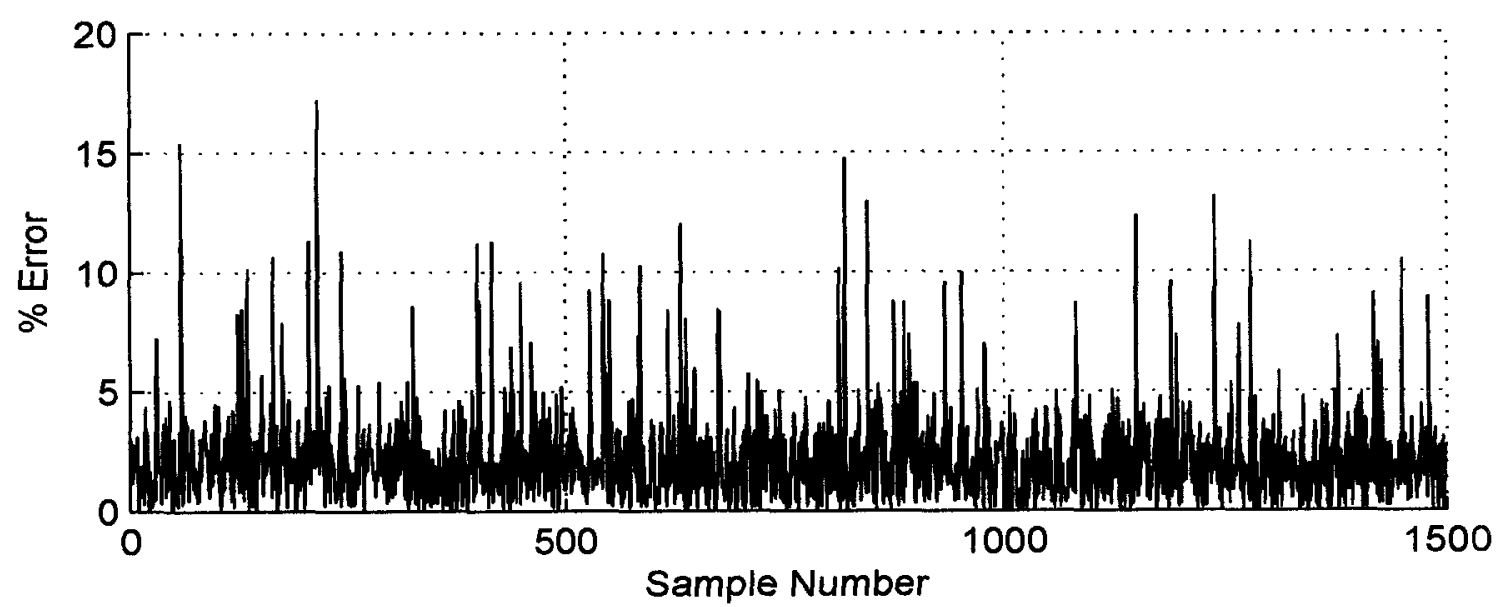

Figure 5.4 (b): Percentage Error of GaN-4 Neural Model with Three-Layer Perceptrons for T-nonlinear Using Back Propagation Training 


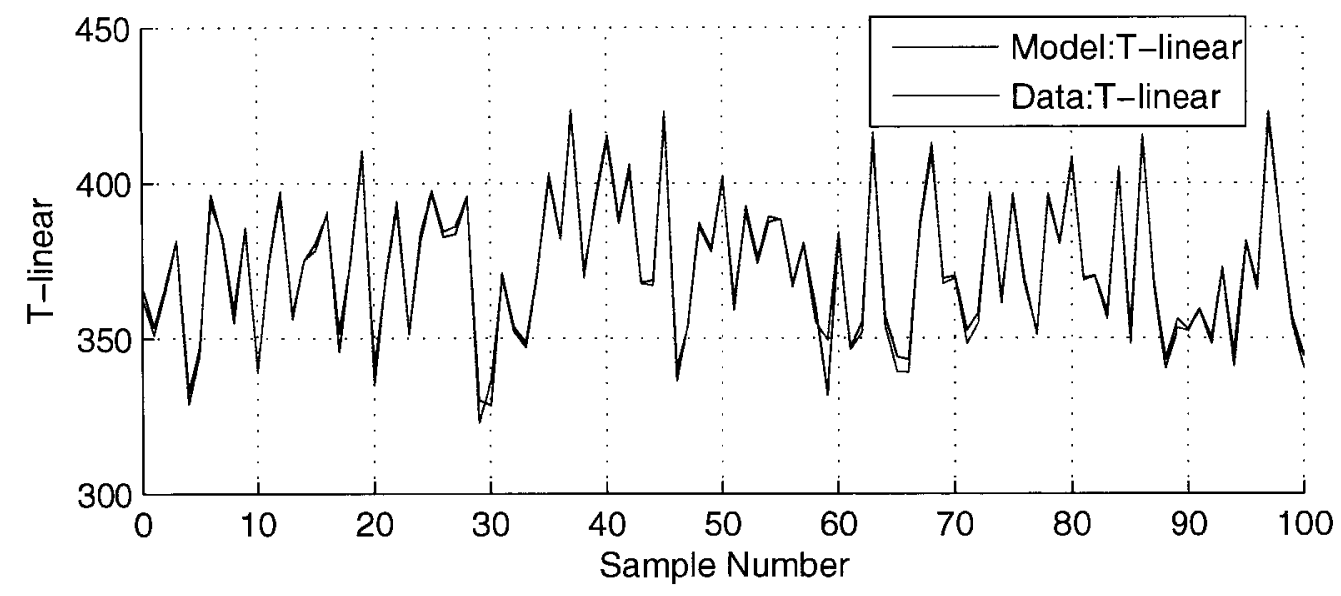

Figure 5.5 (a): Neural Model Output vs. Test Data for T-linear Using Three-Layer GaN-4 Model with Back Propagation Training

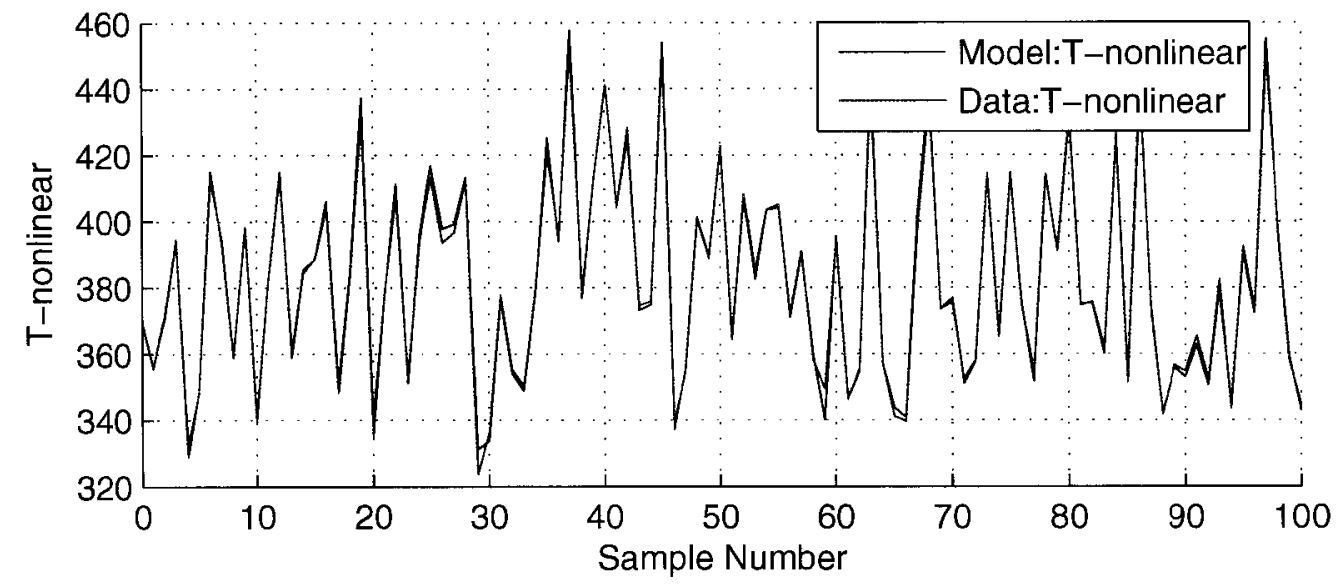

Figure 5.5 (b): Neural Model Output vs. Test Data for T-nonlinear using ThreeLayer GaN-4 Model with Conjugate Gradient Training

Figures 5.5 (a) and (b) show how well the neural model predicts the output using the unseen test data except for the very few samples where the large error occurred. 


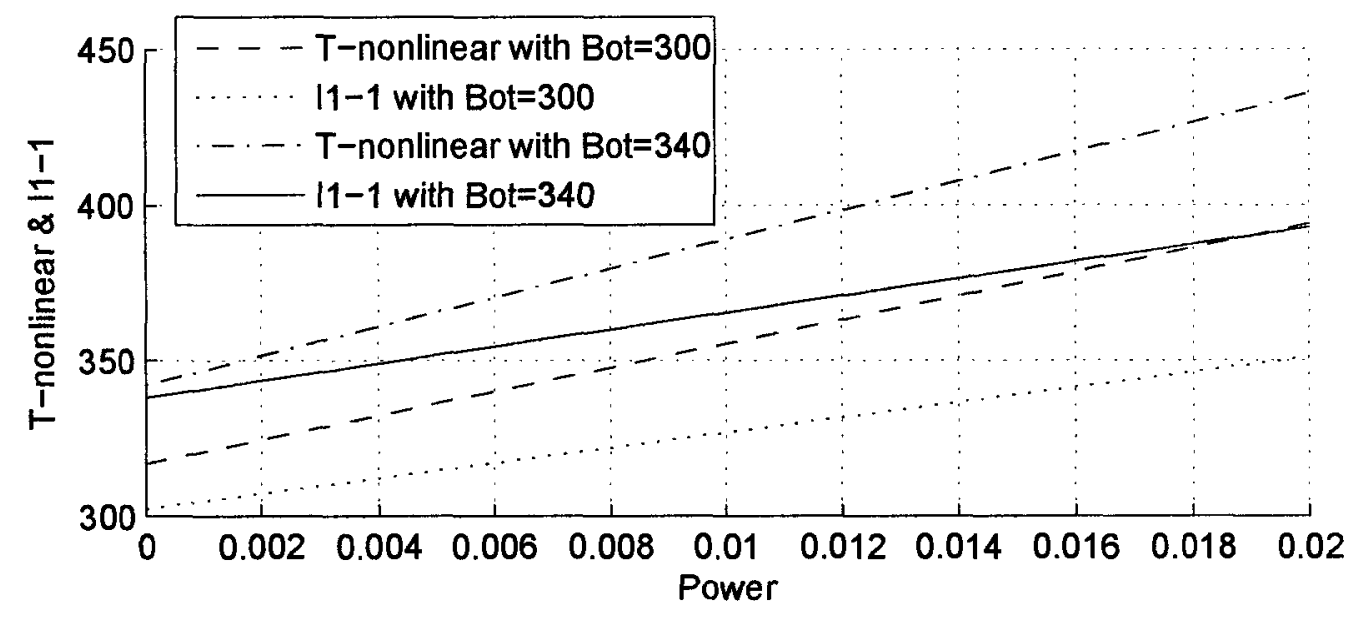

Figure 5.6 (a): T-nonlinear and I1-1 (Outputs) vs. Power (Input) w.r.t. Bot (Input) for Three-Layer GaN-4 Model with Back Propagation Training

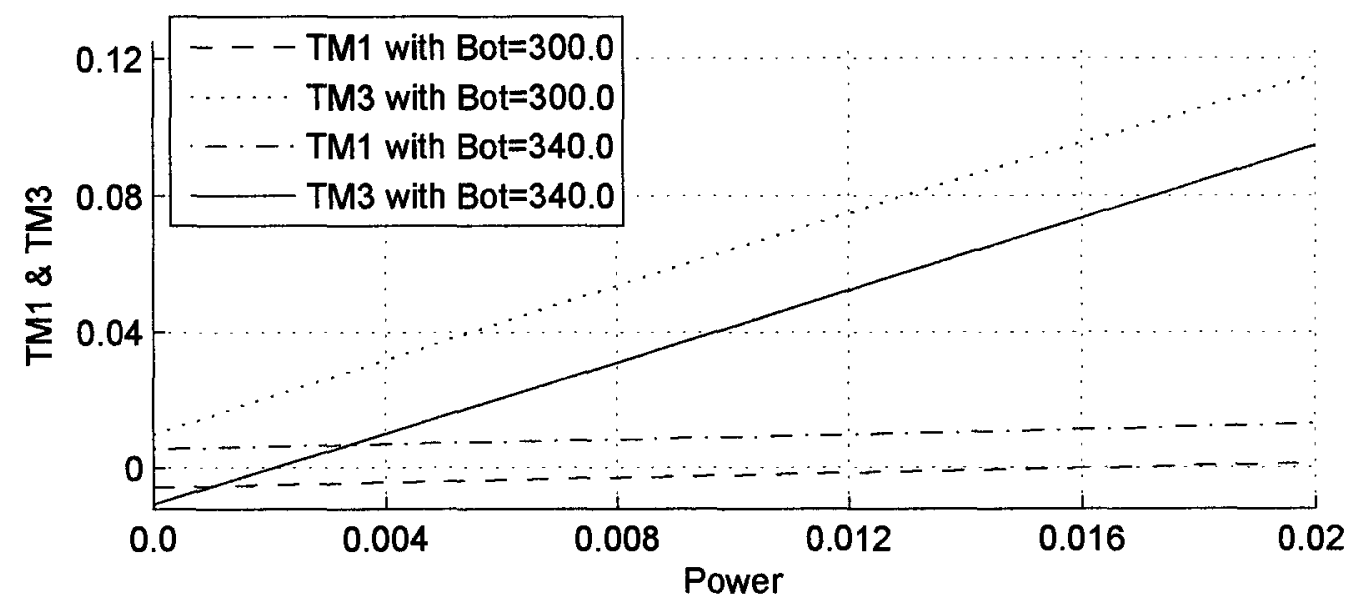

Figure 5.6 (b): TM1 and TM3 (Outputs) vs. Power (Input) w.r.t. Bot (Input) for Three-Layer GaN-4 Model with Conjugate Gradient Training

Figure 5.6 shows the output vs. input plot for this model. The testing errors for four-layer perceptrons network for GaN model are shown in Table B.1.1 in Appendix B. As mentioned earlier, the four-layer perceptrons network has not been well trained for this model. Consequently, the average and maximum errors are comparatively high for some of the outputs of GaN model with four-layer perceptrons as shown in Table B.1.1 in Appendix B. 


\subsubsection{Training of GaN Model with Five-Port Configuration}

The GaN model with five-port configuration has been trained in four ways as follows:

1) Three-layer perceptrons network with back propagation training method.

2) Three-layer perceptrons network with conjugate gradient training method.

3) Four-layer perceptrons network with back propagation training method.

4) Four-layer perceptrons network with conjugate gradient training method.

Table 5.4 shows the training errors for GaN model with five-port configuration.

Table 5.4: Training Errors of GaN Model with Five-Port Configuration

\begin{tabular}{|c|c|c|c|c|}
\hline $\begin{array}{c}\text { Layer/ } \\
\text { Training }\end{array}$ & $\begin{array}{c}\text { Hidden } \\
\text { Neurons }\end{array}$ & Initial Condition & $\begin{array}{c}\text { Initial Training } \\
\text { Error (After 1 } \\
\text { Training) }\end{array}$ & $\begin{array}{c}\text { Final Training } \\
\text { Error (After } \\
\text { 10 }^{\text {th }} \text { Training) }\end{array}$ \\
\hline 3 layer BP & 24 & $\begin{array}{c}\text { max. number of epochs: } 100 \\
\text { learning rate: } 0.001 \\
\text { momentum factor: } 0\end{array}$ & $\begin{array}{c}0.003643798 \\
\text { at } 100 \text { epochs }\end{array}$ & $\begin{array}{c}0.003072574 \\
\text { at } 911 \text { epochs }\end{array}$ \\
\hline 3 layer CG & 24 & $\begin{array}{c}\text { max. number of epochs: } 200 \\
\text { function error tolerance: } 1 \mathrm{e}^{-4} \\
\text { model parameter tolerance: } 1 \mathrm{e}^{-4}\end{array}$ & $\begin{array}{c}0.003507970 \\
\text { at } 179 \text { epochs }\end{array}$ & $\begin{array}{c}0.003497720 \\
\text { at } 188 \text { epochs }\end{array}$ \\
\hline 4 layer BP & layer1: 5 & $\begin{array}{c}\text { max. number of epochs: } 200 \\
\text { learning rate: } 0.001\end{array}$ & $\begin{array}{c}0.019041700 \\
\text { at } 200 \text { epochs }\end{array}$ & $\begin{array}{c}0.018975600 \\
\text { at } 440 \text { epochs }\end{array}$ \\
\hline 4 layer CG & layer1:5 \\
layer2: 3 & $\begin{array}{c}\text { max. number of epochs: } 200 \\
\text { function error tolerance: } 1 \mathrm{e}^{-4} \\
\text { model parameter tolerance: } 1 \mathrm{e}^{-4}\end{array}$ & $\begin{array}{c}0.022101500 \\
\text { at } 154 \text { epochs }\end{array}$ & $\begin{array}{c}0.022080200 \\
\text { at } 167 \text { epochs }\end{array}$ \\
\hline
\end{tabular}

In Table 5.4, the number of hidden neurons and the initial conditions of the trainings have been automatically selected by the Neuromodeler. Figure 5.7 shows the training error of GaN model with five port configuration against the first 500 epochs. 


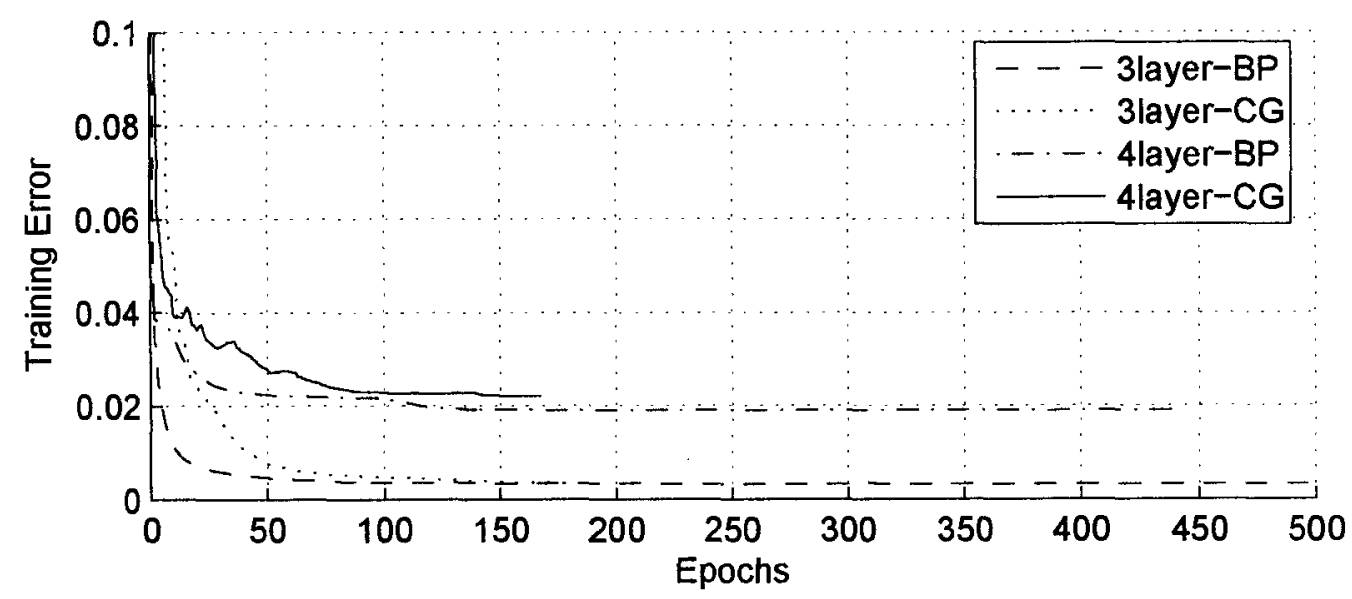
Figure 5.7: Training Error of GaN Model with Five-Port Configuration for
Different Training Methods and Structures

It has been observed from Figure 5.7 that the training error is less for three-layer perceptrons network than that of four-layer perceptrons network for GaN model with five-port configuration.

\subsubsection{Testing of GaN Model with Five-Port Configuration}

Once the neural network was built with the training data, the performance of the network has been tested with a separate set (unseen) of testing data. Table 5.5 shows the training and testing results for $\mathrm{GaN}$ model with five-port configuration.

Table 5.5: Training and Testing Results of GaN Model with Five-Port Configuration

\begin{tabular}{|c|c|c|c|c|c|}
\hline $\begin{array}{c}\text { Result } \\
\text { Training }\end{array}$ & Epochs & $\begin{array}{c}\text { Training } \\
\text { Error }\end{array}$ & $\begin{array}{c}\text { Average } \\
\text { Testing Error }\end{array}$ & $\begin{array}{c}\text { Maximum } \\
\text { Testing Error }\end{array}$ & $\begin{array}{c}\text { Correlation } \\
\text { Coefficient }\end{array}$ \\
\hline 3-layer $B P$ & 911 & 0.003072574 & 3.2704945 & 55.065636 & 0.99974900 \\
\hline 4-layer $B P$ & 188 & 0.018975600 & 3.1327780 & 54.490158 & 0.99977285 \\
\hline 3-layer $C G$ & 440 & 0.003497720 & 3.2883837 & 55.598335 & 0.99974716 \\
\hline 4-layer $C G$ & 167 & 0.022080200 & 2.8269913 & 54.724945 & 0.99984290 \\
\hline
\end{tabular}


It should be mentioned here that the GaN model with five-port configuration was not the optimal choice compared to the four-port configuration as indicated by the simulations performed in chapter 4 . Also, it can be seen from these results that it is difficult to train the five-port model effectively. The average error is satisfactory; however, the maximum error is large for all cases. It appears that the five-port model is not only poor from the point of view of a sub-model definition, but also difficult to train.

Table 5.6 shows the average and maximum testing errors for each of the outputs for $\mathrm{GaN}$ model with three-layer perceptrons. It has been observed from Table 5.6 that the average errors and the maximum errors are within acceptable limit (average error $\leq 5 \%$ and the maximum errors $\leq 15 \%$ ) for all the outputs except TP1-TP5 (port heat-flows) for both back propagation and conjugate gradient training method. These large errors in the heat flows will produce subsequent errors in the solution of any mixed model using this submodel and is most likely not suitable for use. 
Table 5.6: Testing Errors of GaN Model with Three-Layer Perceptrons and FivePort Configuration

\begin{tabular}{|c|c|c|c|c|}
\hline Output & $\begin{array}{c}\text { Average Error } \\
\text { (BP3) }\end{array}$ & $\begin{array}{l}\text { Maximum Error } \\
\text { (BP3) }\end{array}$ & $\begin{array}{l}\text { Average Error } \\
\text { (CG3) }\end{array}$ & $\begin{array}{l}\text { Maximum Error } \\
\text { (CG3) }\end{array}$ \\
\hline$T$-linear & 2.15972850 & 9.2298290 & 2.27445770 & 8.9718740 \\
\hline$T$-nonlinear & 2.29811550 & 8.5067260 & 2.44497630 & 7.2865090 \\
\hline$T P I$ & 15.23984050 & 44.3611530 & 15.33548200 & 43.9338230 \\
\hline$T P 2$ & 16.52241500 & 54.4600260 & 16.57920500 & 53.6577840 \\
\hline$T P 3$ & 17.26269700 & 53.3757100 & 17.33597200 & 53.7503000 \\
\hline$T P 4$ & 17.49096900 & 55.0656360 & 17.52365900 & 55.5983400 \\
\hline TP5 & 46.28382000 & 50,3527070 & 16.34496700 & 50.194145 \\
\hline$I 1-1$ & 1.71833410 & 5.5231786 & 1.74308370 & 5.7716880 \\
\hline$I 1-2$ & 1.54450420 & 6.0625873 & 1.56144010 & 5.5460080 \\
\hline$I 1-3$ & 1.76036400 & 6.0031247 & 1.73555390 & 5.4702454 \\
\hline$I 1-4$ & 1.23968570 & 4.2949850 & 1.23476820 & 3.8861756 \\
\hline$I 1-5$ & 1.15433180 & 4.1855726 & 1.11744630 & 3.9670910 \\
\hline $11-6$ & 1.24651730 & 4.2173047 & 1.22332530 & 4.2373657 \\
\hline$I 2-1$ & 0.65629643 & 2.3617470 & 0.65853570 & 2.2760172 \\
\hline$I 2-2$ & 0.56176406 & 2.1727622 & 0.56966037 & 2.0520031 \\
\hline$I 2-3$ & 0.69446295 & 2.3387194 & 0.67227900 & 2.6447906 \\
\hline$I 2-4$ & 0.77190690 & 2.4907439 & 0.78747670 & 2.5137987 \\
\hline$I 2-5$ & 0.42996117 & 1.5726722 & 0.41901140 & 1.6350898 \\
\hline$I 2-6$ & 0.87606610 & 3.1509798 & 0.87778090 & 3.2556517 \\
\hline $13-1$ & 1.26314970 & 4.0477138 & 1.27031980 & 4.0095960 \\
\hline$I 3-2$ & 0.87014280 & 2.7609124 & 0.88129980 & 2.9948459 \\
\hline$I 3-3$ & 1.37538710 & 4.4899206 & 1.38266060 & 4.5289536 \\
\hline $13-4$ & 1.62167110 & 5.2506433 & 1.63457290 & 4.9991810 \\
\hline$I 3-5$ & 1.17188400 & 3.7345264 & 1.19902150 & 3.8026898 \\
\hline $13-6$ & 1.75507040 & 5.4527493 & 1.77278850 & 5.7172300 \\
\hline$P M I$ & 2.82873400 & 8.6010130 & 2.85109040 & 9.0241970 \\
\hline$P M 2$ & 3.35487600 & 9.7400840 & 3.38093350 & 9.4547760 \\
\hline$P M 3$ & 3.91800380 & 11.4465820 & 3.94236700 & 11.1299340 \\
\hline$P B 1$ & 2.91111970 & 8.7053950 & 2.90949800 & 8.5569935 \\
\hline$P B 2$ & 3.02547740 & 9.0862060 & 3.03168230 & 9.1865280 \\
\hline$P B 3$ & 2.99874780 & 9.1148405 & 3.02561140 & 9.0056270 \\
\hline$P B 4$ & 3.37262060 & 9.3556150 & 3.38295220 & 9.7333100 \\
\hline$P l$ & 1.56043670 & 5.8935120 & 1.55252500 & 5.4855480 \\
\hline$P 2$ & 1.16651950 & 4.3430867 & 1.13764100 & 3.8920557 \\
\hline P3 & 0.57099026 & 2.2641394 & 0.56479000 & 2.1393354 \\
\hline$P 4$ & 0.44566480 & 1.5894451 & 0.45877773 & 1.6813142 \\
\hline$P 5$ & 0.87627960 & 2.6808000 & 0.88039255 & 2.9385588 \\
\hline$P 6$ & 1.18873140 & 3.6988410 & 1.20522210 & 3.8243818 \\
\hline$P 7$ & 1.75840300 & 5.5204870 & 1.74836130 & 5.2982388 \\
\hline$P 8$ & 1.23599370 & 3.9630525 & 1.22817720 & 3.9857590 \\
\hline$P 9$ & 0.69552430 & 2.5355825 & 0.70698017 & 2.5374393 \\
\hline$P 10$ & 0.90821780 & 3.1399927 & 0.92098075 & 3.2287571 \\
\hline$P 11$ & 1.38333690 & 4.5021550 & 1.40505080 & 4.5511312 \\
\hline$P 12$ & 1.73299200 & 5.4227520 & 1.77610460 & 5.7185626 \\
\hline
\end{tabular}


Figure 5.8 shows the testing error plot for TP1 for GaN model with three-layer perceptrons network.

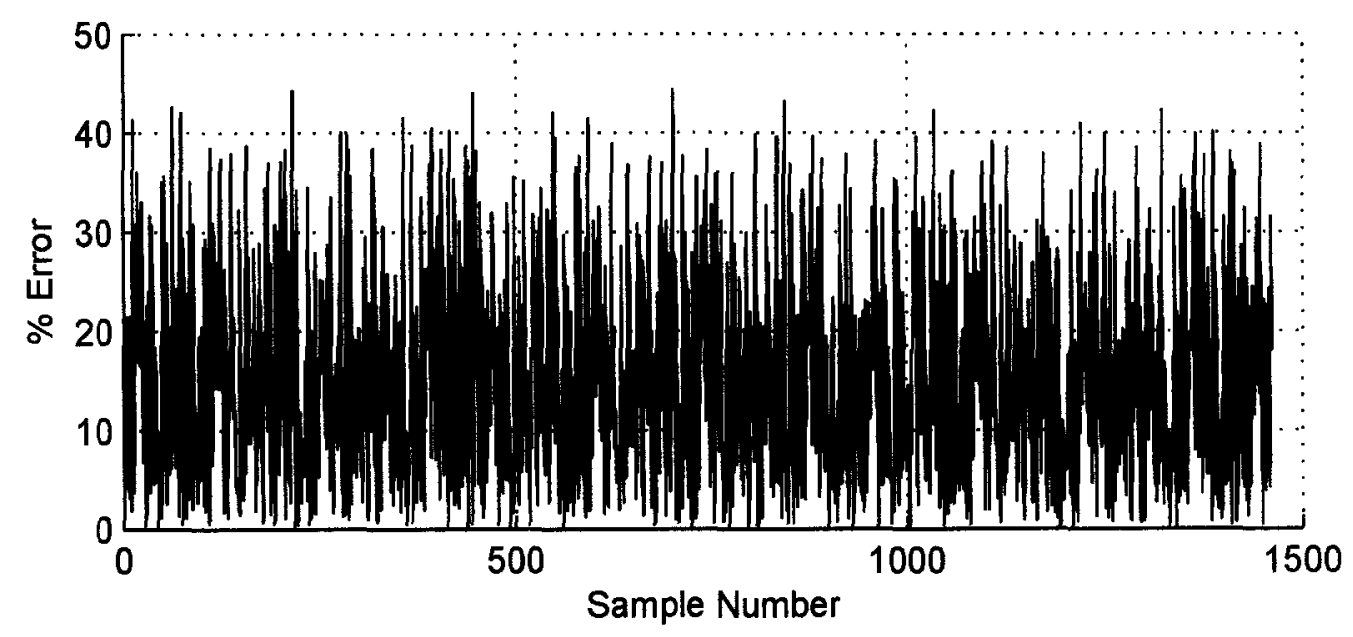

Figure 5.8: Percentage Error of Neural Model for TP1 Using Three-Layer GaN-5 Model with Back Propagation Training

The output vs. input plots for this model are shown in Figure 5.9 (a) and Figure 5.9 (b).

These two figures indicate the non-linearity present in the model. The testing errors for four-layer perceptrons network are shown in Table B.1.2 in Appendix B. As mentioned earlier, the four-layer perceptrons network has not been well trained for this model. Consequently, the average and maximum errors are comparatively high for some of the outputs of GaN model with four-layer perceptrons as shown in Table B.1.2 in Appendix B. 


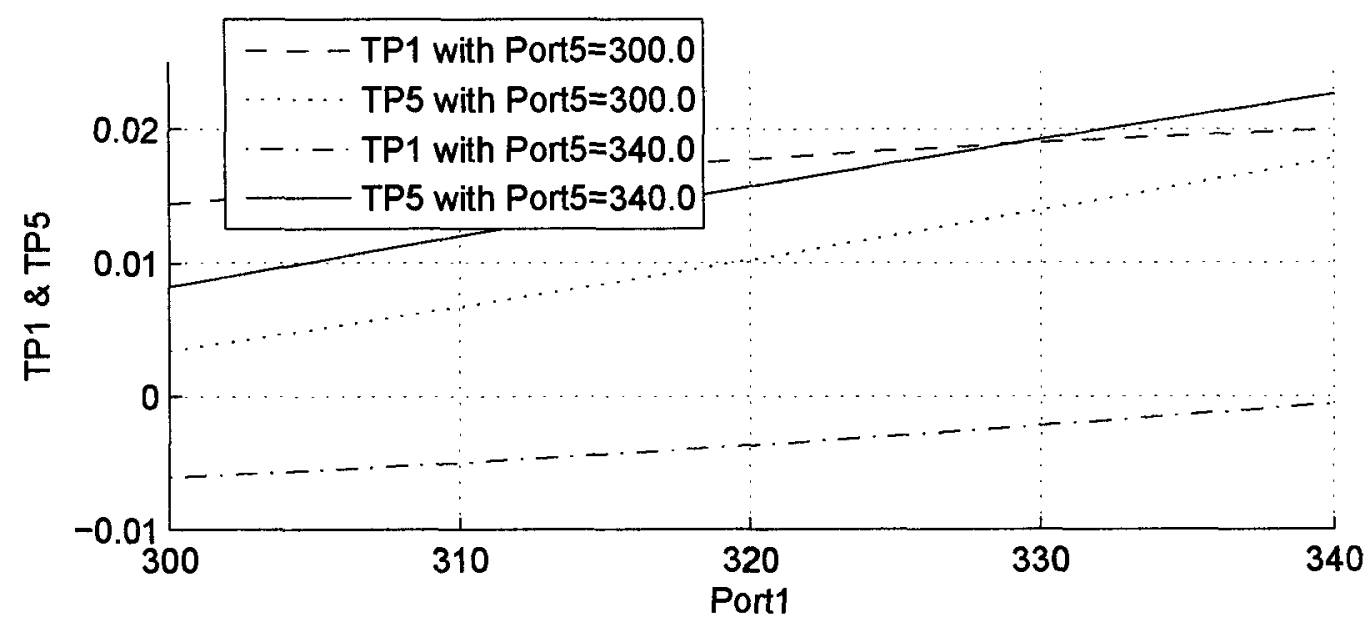

Figure 5.9 (a): TP1 and TP5 (Outputs) vs. Port1 (Input) w.r.t Port5 (Input) for Three-Layer GaN-5 Model with Back Propagation Training

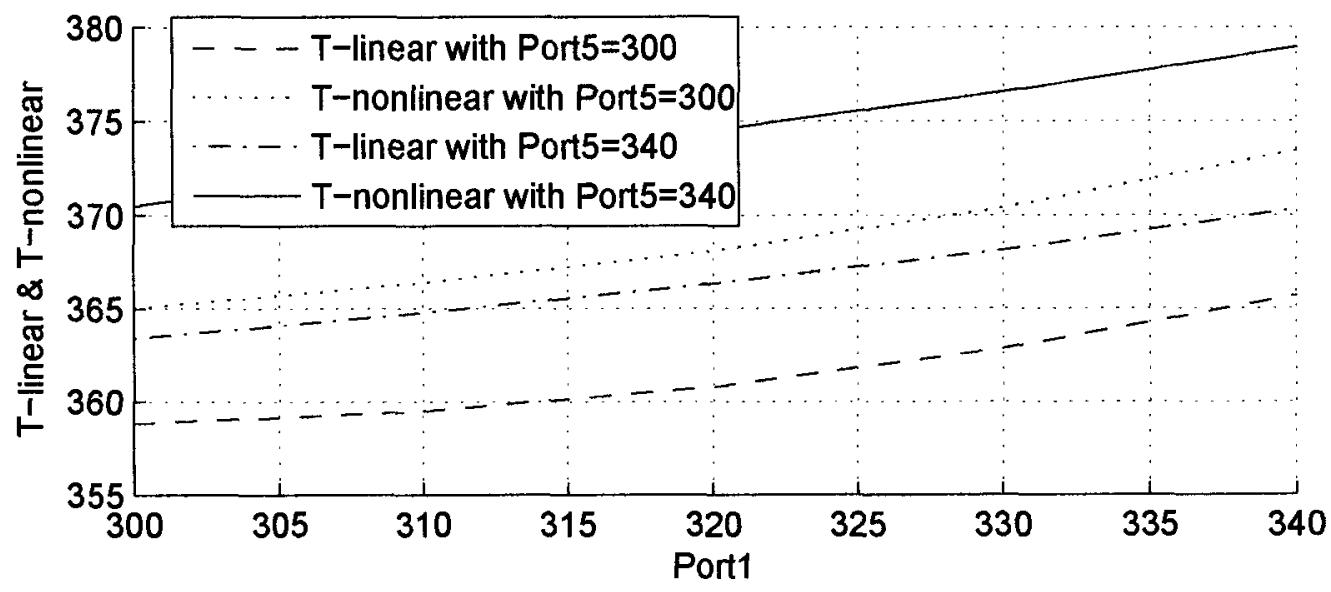

Figure 5.9 (b): T-linear and T-nonlinear (Outputs) vs. Port1 (Input) w.r.t Port5 (Input) for Three-Layer GaN-5 Model with Conjugate Gradient Training 


\subsection{GaAs Based Power Amplifier}

As mentioned in Chapter 4, the five-port configuration has been selected as the appropriate choice for neural network modeling for GaAs based power amplifier model. The inputs for the neural network model have been varied as follows: port1 (port at the bottom) temperature has been varied from $300-330 \mathrm{~K}$ (with an increase of $15 \mathrm{~K}$ ), port 2 to port5 (four metal ports) temperatures have been varied from $300-345 \mathrm{~K}$ (with an increase of $15 \mathrm{~K}$ ) and power has been varied from $0-0.01 \mathrm{~W}$ (with an increase of $0.0025 \mathrm{~W})$. The outputs of this model include the maximum temperature (T-linear) of a simple linear solution of the model, maximum temperature (T-nonlinear) of a non-linear solution, port heat-flows (TP1-TP5) for each of the input ports and temperatures at 21 different points that might be of interest. A total of 3840 data (where each data represents a set of all the inputs and outputs) have been generated for training. The performance of the trained model has been evaluated by a total of 1500 data.

\subsubsection{Training of GaAs Based Power Amplifier}

The model has been trained in four different ways as follows:

1) Three-layer perceptrons network with back propagation training method.

2) Three-layer perceptrons network with conjugate gradient training method.

3) Four-layer perceptrons network with back propagation training method.

4) Four-layer perceptrons network with conjugate gradient training method. 
Table 5.7 shows the summary of training errors for GaAs model. In this table, the number of hidden neurons and the initial conditions of the trainings have been automatically selected by the Neuromodeler.

Table 5.7: Training Errors of GaAs Model

\begin{tabular}{|c|c|c|c|c|}
\hline $\begin{array}{c}\text { Layer/ } \\
\text { Training }\end{array}$ & $\begin{array}{c}\text { Hidden } \\
\text { Neurons }\end{array}$ & Initial Condition & $\begin{array}{c}\text { Initial Training } \\
\text { Error (After 1 } \\
\text { Training) }\end{array}$ & $\begin{array}{c}\text { Final Training } \\
\text { Error (After } \\
\text { 10 }^{\text {th }} \text { Training) }\end{array}$ \\
\hline 3 layer BP & 24 & $\begin{array}{c}\text { max. number of epochs: } 100 \\
\text { learning rate: } 0.001 \\
\text { momentum factor: } 0\end{array}$ & $\begin{array}{c}0.01096288 \\
\text { at } 100 \text { epochs }\end{array}$ & $\begin{array}{c}0.005339213 \\
\text { at } 1000 \text { epochs }\end{array}$ \\
\hline 3 layer CG & 24 & $\begin{array}{c}\text { max. number of epochs: } 200 \\
\text { function error tolerance: } 1 \mathrm{e}^{-4} \\
\text { model parameter tolerance: } 1 \mathrm{e}^{-4}\end{array}$ & $\begin{array}{c}0.00494035 \\
\text { at } 100 \text { epochs }\end{array}$ & $\begin{array}{c}0.004048710 \\
\text { at } 413 \text { epochs }\end{array}$ \\
\hline 4 layer BP & $\begin{array}{l}\text { layer1:5 } \\
\text { layer2: 3 }\end{array}$ & $\begin{array}{c}\text { max. number of epochs: } 200 \\
\text { learning rate: } 0.25 \\
\text { momentum factor: } 0\end{array}$ & $\begin{array}{c}0.06864810 \\
\text { at } 200 \text { epochs }\end{array}$ & $\begin{array}{c}0.065361600 \\
\text { at } 817 \text { epochs }\end{array}$ \\
\hline 4 layer CG & layer1:5 & $\begin{array}{c}\text { max. number of epochs: } 200 \\
\text { function error tolerance: } 1 \mathrm{e}^{-4} \\
\text { layer2: } 3\end{array}$ & $\begin{array}{c}0.10534600 \\
\text { at } 58 \text { epochs }\end{array}$ & $\begin{array}{c}0.070714500 \\
\text { at } 150 \text { epochs }\end{array}$ \\
\hline
\end{tabular}

Figure 5.10 shows the training error against the first 500 epochs.

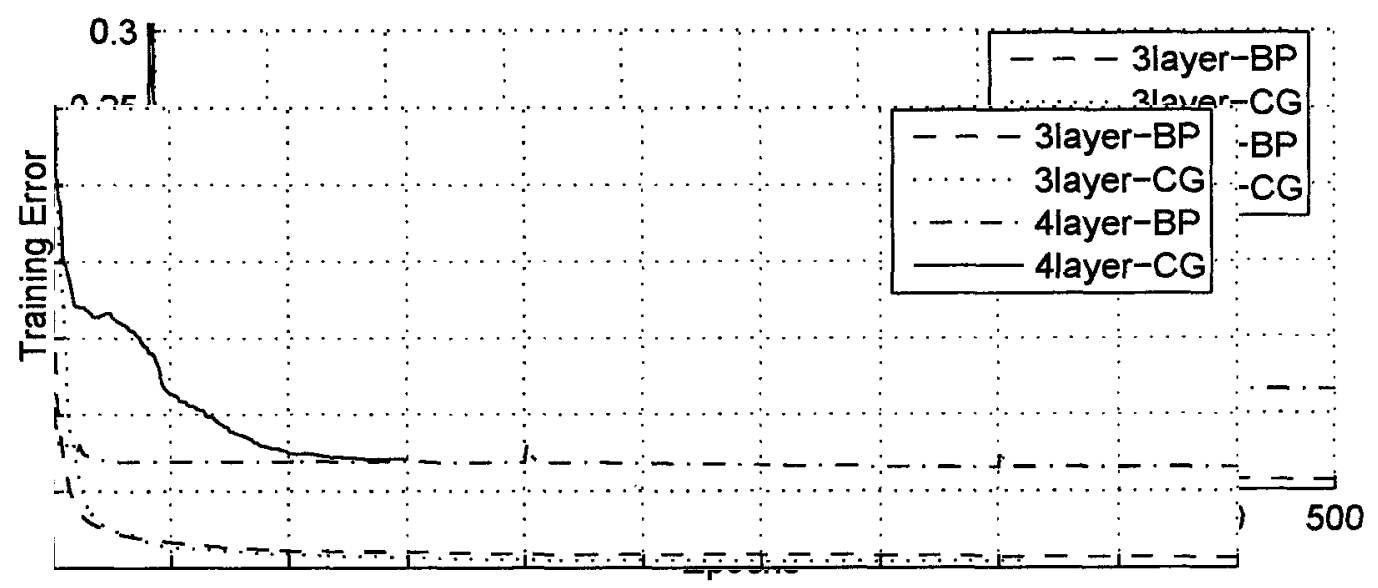

Figure 5.10: Training Error of GaAs Model with Five-Port Configuration for Different Training Methods and Structures 
It has been observed from Figure 5.10 that at any epoch, the training error is less for three-layer perceptrons network than that of four-layer perceptrons network.

\subsubsection{Testing of GaAs Based Power Amplifier}

Once the neural network was built with the training data, the performance of the network has been tested with a separate set (unseen) of testing data. Table 5.8 shows the training and testing results for GaAs model with five-port configuration.

Table 5.8: Training and Testing Results for GaAs Model

\begin{tabular}{|c|c|c|c|c|c|}
\hline $\begin{array}{c}\text { Result } \\
\text { Training }\end{array}$ & Epochs & $\begin{array}{c}\text { Training } \\
\text { Error }\end{array}$ & $\begin{array}{c}\text { Average } \\
\text { Testing Error }\end{array}$ & $\begin{array}{c}\text { Maximum } \\
\text { Testing Error }\end{array}$ & $\begin{array}{c}\text { Correlation } \\
\text { Coefficient }\end{array}$ \\
\hline 3-layer $B P$ & 1000 & 0.005339213 & 0.5503954 & 13.041626 & 0.99999386 \\
\hline 4-layer $B P$ & 413 & 0.065361600 & 5.1452947 & 55.120190 & 0.99990220 \\
\hline 3-layer $C G$ & 817 & 0.004048710 & 0.5462286 & 13.099299 & 0.99999547 \\
\hline 4-layer $C G$ & 150 & 0.070714500 & 5.5548210 & 54.780457 & 0.99990046 \\
\hline
\end{tabular}

It can be observed from Table 5.8 that the average testing errors and maximum testing errors are less and correlation coefficients are higher for models with three-layer perceptrons network than that of four-layer perceptrons network. Therefore, it can be concluded that the model is well trained with conjugate gradient training as well as back propagation training method for three-layer perceptrons network.

Table 5.9 shows the average and maximum testing errors for each of the outputs for GaAs model with three-layer perceptrons. 
Table 5.9: Testing Errors of GaAs Model with Three-Layer Perceptrons

\begin{tabular}{|c|c|c|c|c|}
\hline Output & $\begin{array}{c}\text { Average Error } \\
\text { (BP3) }\end{array}$ & $\begin{array}{c}\text { Maximum Error } \\
\text { (BP3) }\end{array}$ & $\begin{array}{c}\text { Average Error } \\
\text { (CG3) }\end{array}$ & $\begin{array}{c}\text { Maximum Error } \\
\text { (CG3) }\end{array}$ \\
\hline$T$-linear & 2.25731470 & 13.0416360 & 1.97224880 & 13.09930000 \\
\hline$T$-nonlinear & 1.79904580 & 10.6978610 & 1.45902140 & 8.94953800 \\
\hline$T P 1$ & 0.57886840 & 1.5743921 & 0.59930650 & 1.40613060 \\
\hline$T P 2$ & 0.35232836 & 1.2966421 & 0.25267416 & 0.86466426 \\
\hline$T P 3$ & 0.35248274 & 1.1821772 & 0.20652726 & 1.48224310 \\
\hline$T P 4$ & 0.42490458 & 1.6332222 & 0.29367290 & 0.94798980 \\
\hline$T P 5$ & 0.31927323 & 1.1493973 & 0.23900111 & 0.75720390 \\
\hline$I 1-1$ & 0.43714833 & 1.7034156 & 0.24551924 & 0.74990773 \\
\hline$I 1-2$ & 0.40407280 & 1.4732306 & 0.29216793 & 1.00121990 \\
\hline$I 1-3$ & 0.44717246 & 1.8181883 & 0.29090983 & 1.00294960 \\
\hline$I 2-1$ & 0.48667547 & 1.7483943 & 0.26951233 & 0.99097687 \\
\hline$I 2-2$ & 0.46217000 & 1.5806475 & 0.32884246 & 1.09387120 \\
\hline$I 2-3$ & 0.43428900 & 1.6779274 & 0.29080160 & 1.14267250 \\
\hline$I 3-1$ & 0.42596188 & 1.5146278 & 0.15763140 & 0.82151645 \\
\hline$I 3-2$ & 0.37499340 & 1.1323408 & 0.37822070 & 1.47117380 \\
\hline$I 3-3$ & 0.34860190 & 1.3983166 & 0.29815072 & 1.22873160 \\
\hline$I 4-1$ & 0.42374230 & 1.5067503 & 0.34187130 & 1.05552090 \\
\hline$I 4-2$ & 0.42517465 & 1.6732249 & 0.29112430 & 1.01818970 \\
\hline$I 4-3$ & 0.35762840 & 1.3381972 & 0.28807318 & 0.94420010 \\
\hline$P M 1$ & 0.50909954 & 1.9942312 & 0.36783135 & 1.27201190 \\
\hline$P M 2$ & 0.42854570 & 1.4249014 & 0.41840208 & 1.44194270 \\
\hline$P M 3$ & 0.44432476 & 1.5516003 & 0.34155172 & 1.25239920 \\
\hline$P M 4$ & 0.40518090 & 1.4924512 & 0.39142040 & 1.39261150 \\
\hline$P B 1$ & 0.57582060 & 1.5247957 & 1.02286890 & 1.88858410 \\
\hline$P B 2$ & 0.34980650 & 1.0355856 & 1.13225270 & 2.08211260 \\
\hline$P B 3$ & 0.47552297 & 1.4614894 & 1.12922170 & 2.20019300 \\
\hline$P B 4$ & 0.60729500 & 2.0441377 & 0.95298970 & 1.92422180 \\
\hline$P B 5$ & 0.50362550 & 1.3863567 & 1.04258850 & 2.06928900 \\
\hline & & & & \\
\hline
\end{tabular}

It has been observed from Table 5.9 that the average errors as well as the maximum errors are within acceptable limit i.e., average error $\leq 5 \%$ and the maximum errors $\leq$ $15 \%$. It can be concluded that the model with three-layer perceptrons network is well trained with back propagation as well as conjugate gradient training method. Figure 5.11 (a) and Figure 5.11 (b) show the testing error plots for T-linear with conjugate gradient and T-nonlinear with back propagation training method respectively for three-layer perceptrons network. 


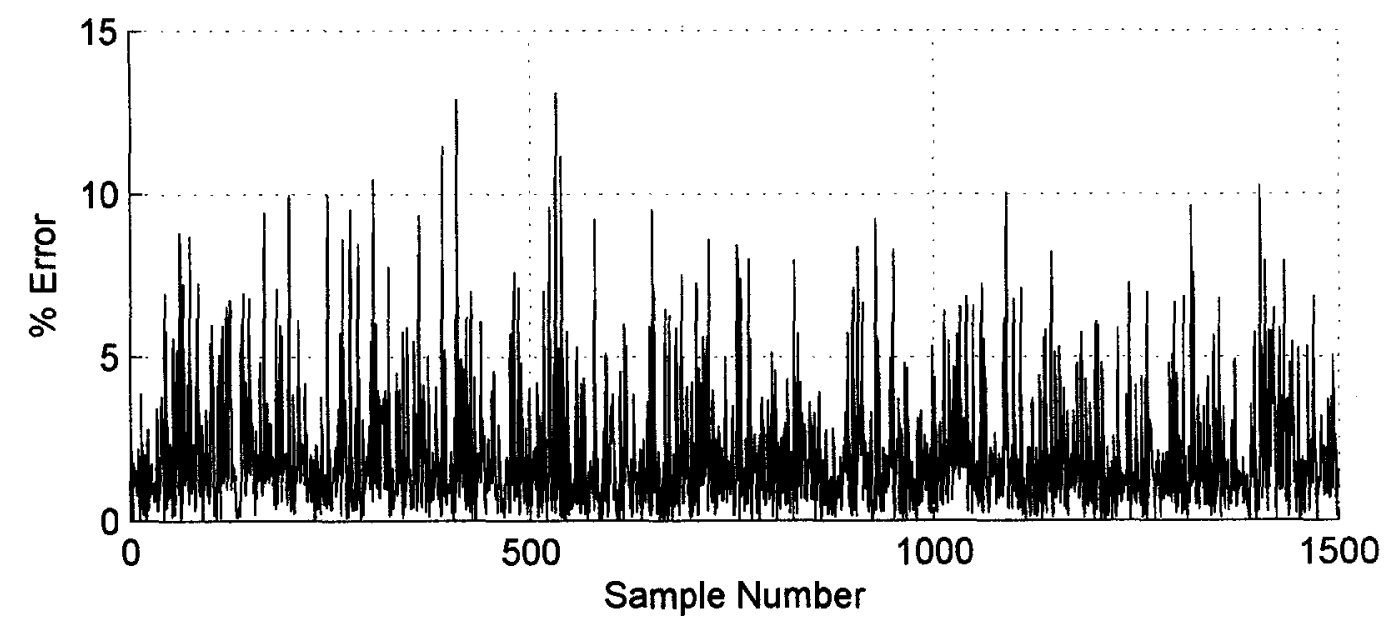

Figure 5.11 (a): Percentage Error of Neural Model for T-linear Using Three-Layer GaAs Model with Conjugate Gradient Training

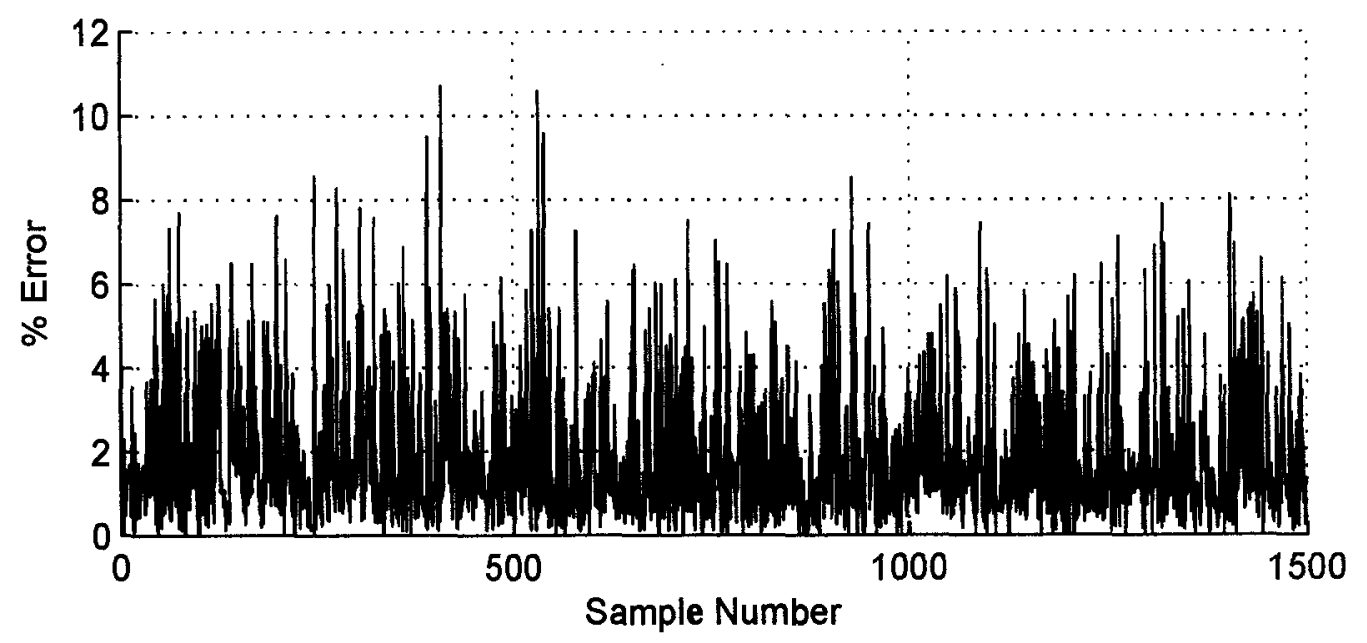

Figure 5.11 (b): Percentage Error of Neural Model for T-nonlinear Using ThreeLayer GaAs Model with Back Propagation Training 
Figure 5.12 (a) shows T-nonlinear vs. sample number and Figure 5.12 (b) shows TP1 vs. sample number plots for back propagation and conjugate gradient training method respectively. These plots show how well the neural model predicts the output using the unseen test data.

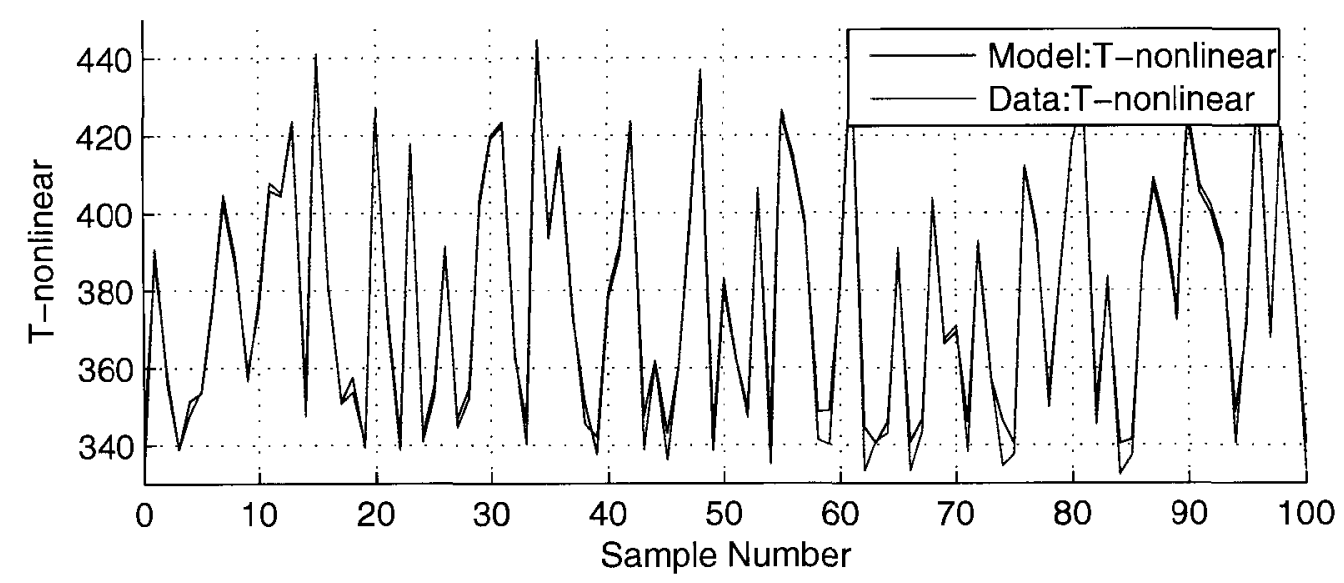

Figure 5.12 (a): Neural Model Output vs. Test Data for T-nonlinear Using ThreeLayer GaAs Model with Back Propagation Training

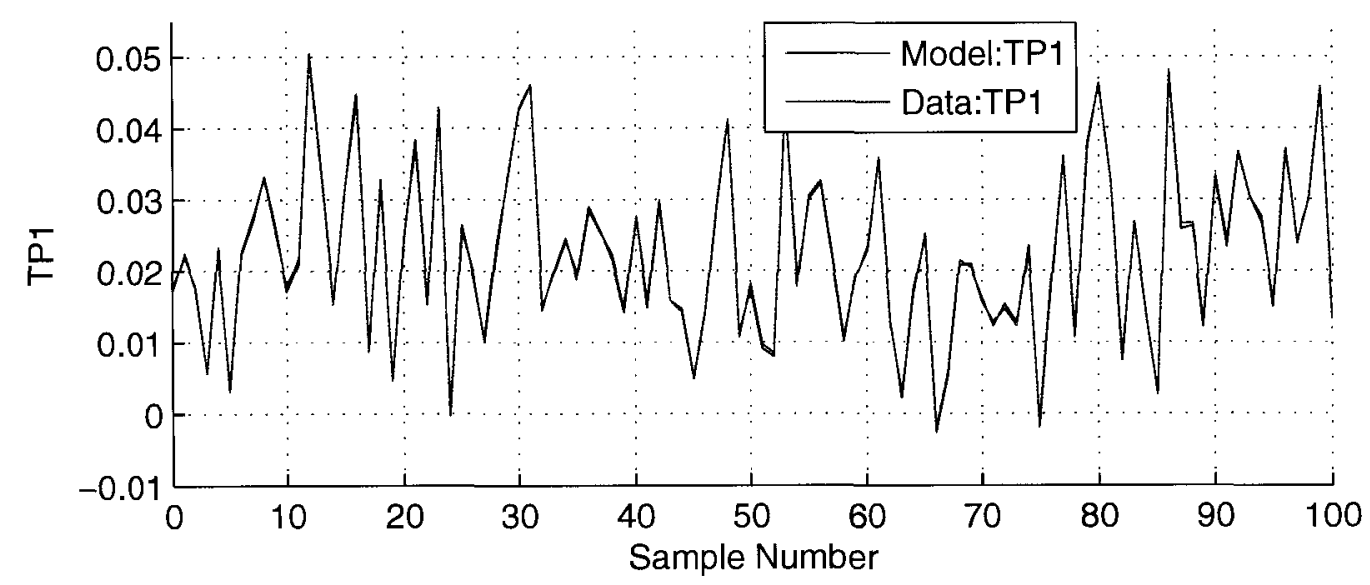

Figure 5.12 (b): Neural Model Output vs. Test Data for TP1 Using Three-Layer GaAs Model with Conjugate Gradient Training 
Figure 5.13 (a) and Figure 5.13 (b) show the output vs. input plot for GaAs model with three-layer perceptrons network. These figures indicate the non-linearity present in the model. The testing errors for four-layer perceptrons network are shown in Table B.2.1 in Appendix B. As mentioned earlier, the four-layer perceptrons network has not been well trained for this model. Consequently, the average and maximum errors are comparatively high for some of the outputs of GaAs model with four-layer perceptrons.

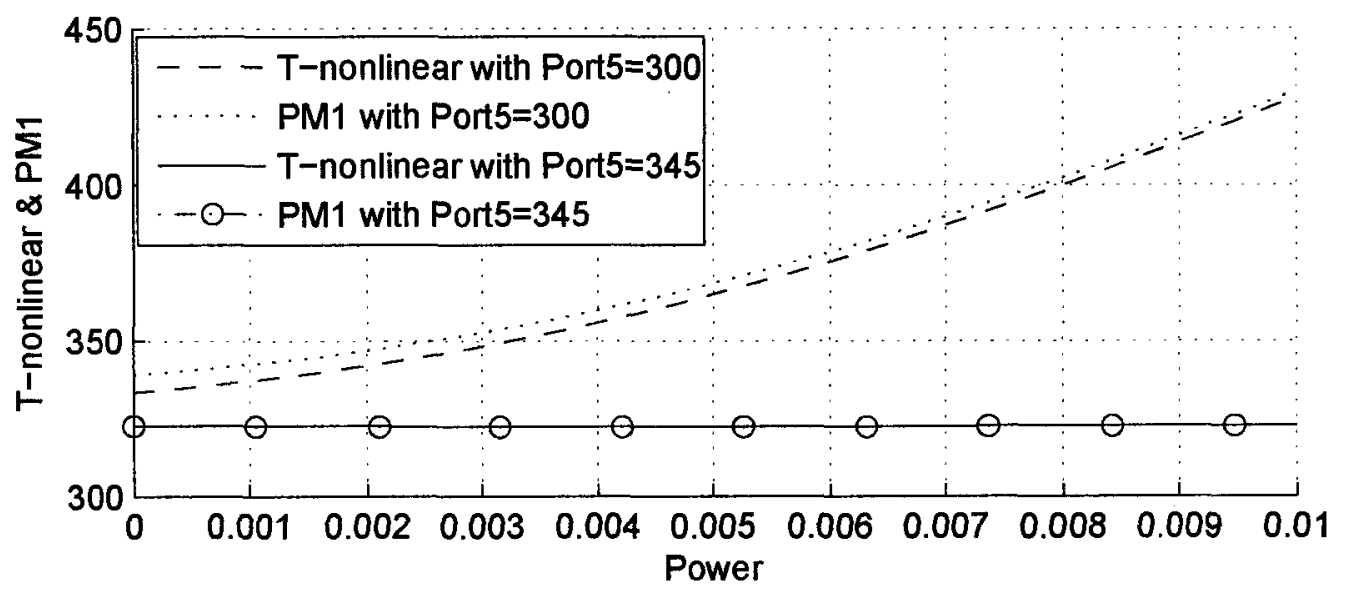

Figure 5.13 (a): T-nonlinear and PM1 (Outputs) vs. Power (Input) w.r.t Port5 (Input) for Three-Layer GaAs Model with Back Propagation Training

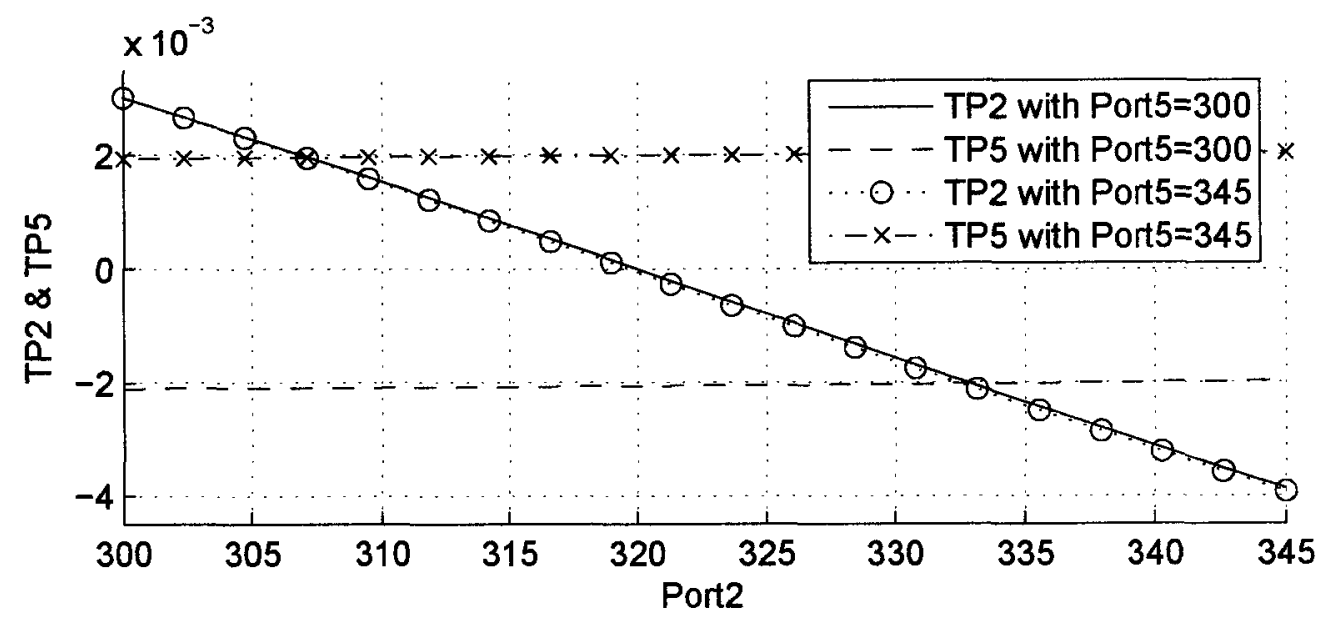

Figure 5.13 (b): TP2 and TP5 (Outputs) vs. Port2 (Input) w.r.t Port5 (Input) for Three-Layer GaAs Model with Conjugate Gradient Training 


\subsection{Package Model}

The results obtained from the simulation using Atar shows that the three-port configuration gives best results for this model. Accordingly, the inputs chosen for this model for neural network modeling are as follows: the heat-transfer coefficient in the topside of the model (alpha-top), the three port temperatures $(T 1, T 2$ and $T 3)$ and the power. The value of alpha-top has been chosen as, $10^{-4}-10^{-8} \mathrm{~W} / \mu \mathrm{m}^{2} \mathrm{~K}$ (with a decrease of $10^{-1}$ $\mathrm{W} / \mu \mathrm{m}^{2} \mathrm{~K}$ ), using log scale. The temperatures have been varied from $300-340 \mathrm{~K}$ (with an increase of $10 \mathrm{~K}$ ) while the power has been varied as $1.0-0 \mathrm{~W}$ (with a decrease of $0.25 \mathrm{~W}$ ). The outputs of this model include the maximum temperature (T-linear) of a simple linear solution of the model, maximum temperature (T-nonlinear) of a non-linear solution, port heat-flow (T-alpha) due to alpha, port heat-flows (TP1-TP3) for each of the input ports and temperatures at 16 different points that might be of interest. A total of approximately 3100 data (where each data represents a set of all the inputs and outputs) have been generated for training. The performance of the trained model has been evaluated by a total of 1500 data.

\subsubsection{Training of Package Model}

The model has been trained in four different ways as follows:

1) Three-layer perceptrons network with back propagation training method.

2) Three-layer perceptrons network with conjugate gradient training method.

3) Four-layer perceptrons network with back propagation training method. 
4) Four-layer perceptrons network with conjugate gradient training method.

Table 5.10 shows the summary of training errors for Package model. In this table, the number of hidden neurons and the initial conditions of the trainings have been automatically selected by the Neuromodeler. Figure 5.14 shows the training error against the first 300 epochs.

Table 5.10: Training Errors of Package Model

\begin{tabular}{|c|c|c|c|c|}
\hline $\begin{array}{c}\text { Layer/ } \\
\text { Training }\end{array}$ & $\begin{array}{c}\text { Hidden } \\
\text { Neurons }\end{array}$ & Initial Condition & $\begin{array}{c}\text { Initial Training } \\
\text { Error (After 1 } \\
\text { Training) }\end{array}$ & $\begin{array}{c}\text { Final Training } \\
\text { Error (After } \\
\text { 10 }^{\text {th }} \text { Training) }\end{array}$ \\
\hline 3 layer BP & 20 & $\begin{array}{c}\text { max. number of epochs: } 100 \\
\text { learning rate: } 0.25 \\
\text { momentum factor: } 0\end{array}$ & $\begin{array}{c}0.007269213 \\
\text { at } 100 \text { epochs }\end{array}$ & $\begin{array}{c}0.005346793 \\
\text { at } 1000 \text { epochs }\end{array}$ \\
\hline 3 layer CG & 20 & $\begin{array}{c}\text { max. number of epochs : } 200 \\
\text { function error tolerance: } 1 \mathrm{e}^{-4} \\
\text { model parameter tolerance: } 1 \mathrm{e}^{-4}\end{array}$ & $\begin{array}{c}0.011737000 \\
\text { at } 100 \text { epochs }\end{array}$ & $\begin{array}{c}0.006601130 \\
\text { at } 619 \text { epochs }\end{array}$ \\
\hline 4 layer BP & $\begin{array}{l}\text { layer1: 5 } \\
\text { layer2: 3 }\end{array}$ & $\begin{array}{c}\text { max. number of epochs: } 200 \\
\text { learning rate: } 0.25 \\
\text { momentum factor: 0 }\end{array}$ & $\begin{array}{c}0.029792300 \\
\text { at } 200 \text { epochs }\end{array}$ & $\begin{array}{c}0.028774800 \\
\text { at } 1073 \text { epochs }\end{array}$ \\
\hline 4 layer CG & $\begin{array}{l}\text { layer1:5 } \\
\text { layer2: 3 }\end{array}$ & $\begin{array}{c}\text { max. number of epochs: } 200 \\
\text { function error tolerance: } 1 \mathrm{e}^{-4} \\
\text { model parameter tolerance: } 1 \mathrm{e}^{-4}\end{array}$ & $\begin{array}{c}0.053253600 \\
\text { at } 44 \text { epochs }\end{array}$ & $\begin{array}{c}0.031112200 \\
\text { at } 253 \text { epochs }\end{array}$ \\
\hline
\end{tabular}

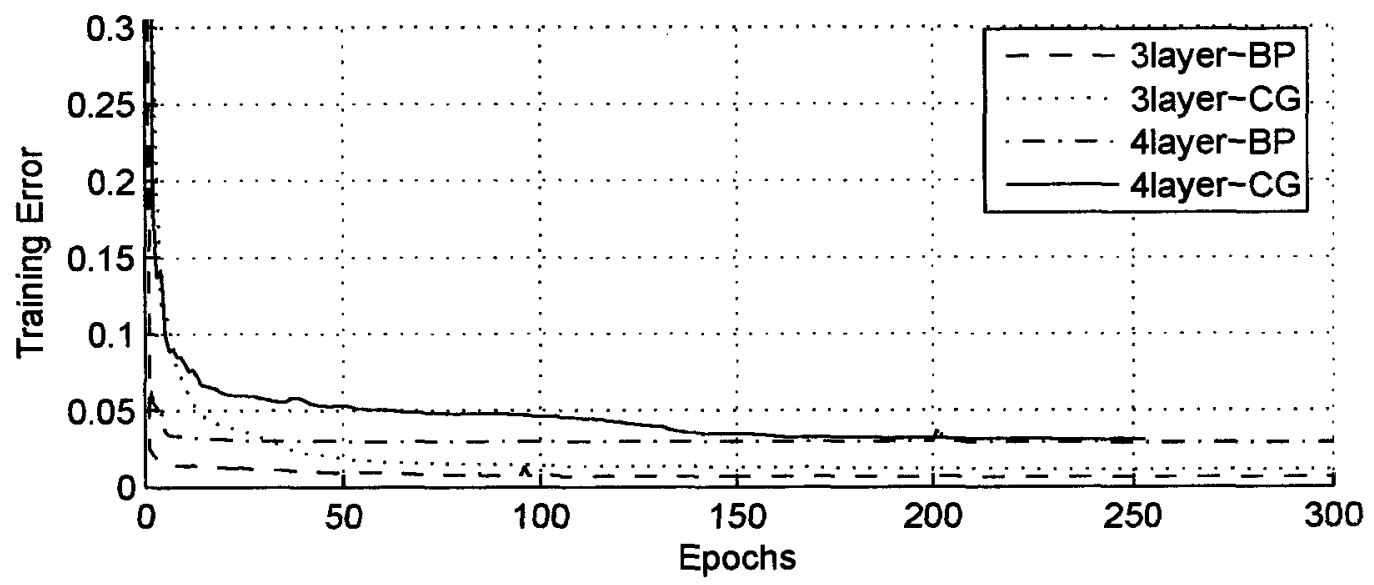

Figure 5.14: Training Error of Package Model with Three-Port Configuration for Different Training Methods and Structures 
It has been observed from Figure 5.14 that the training error is less for three-layer perceptrons network than that of four-layer perceptrons network.

\subsubsection{Testing of Package Model}

Once the neural network was built with the training data, the performance of the network has been tested with a separate set (unseen) of testing data. Table 5.11 shows the training and testing results for Package model with three-port configuration.

Table 5.11: Training and Testing Results for Package Model

\begin{tabular}{|c|c|c|c|c|c|}
\hline $\begin{array}{c}\text { Result } \\
\text { Training }\end{array}$ & Epochs & $\begin{array}{c}\text { Training } \\
\text { Error }\end{array}$ & $\begin{array}{c}\text { Average } \\
\text { Testing Error }\end{array}$ & $\begin{array}{c}\text { Maximum } \\
\text { Testing Error }\end{array}$ & $\begin{array}{c}\text { Correlation } \\
\text { Coefficient }\end{array}$ \\
\hline 3-layer $B P$ & 1000 & 0.005346793 & 0.77947116 & 8.4207680 & 0.99999740 \\
\hline 4-layer $B P$ & 619 & 0.028774800 & 2.43547370 & 26.2361720 & 0.99997896 \\
\hline 3-layer $C G$ & 1073 & 0.006601130 & 0.87973390 & 7.1821856 & 0.99999720 \\
\hline 4-layer $C G$ & 253 & 0.031112200 & 2.78219400 & 28.0604290 & 0.99997720 \\
\hline
\end{tabular}

It can be observed from Table 5.11 that the average testing errors and maximum testing errors are also less and correlation coefficients are higher for models with three-layer perceptrons network than that of four-layer perceptrons network. Therefore, it can be concluded that the model is well trained with conjugate gradient training as well as back propagation training method for three-layer perceptrons network.

Table 5.12 shows the average and maximum testing errors for each of the outputs for Package model with three-layer perceptrons. 
Table 5.12: Testing Errors of Package Model with Three-Layer Perceptrons

\begin{tabular}{|c|c|c|c|c|}
\hline Output & $\begin{array}{c}\text { Average Error } \\
\text { (BP3) }\end{array}$ & $\begin{array}{c}\text { Maximum } \\
\text { Error (BP3) }\end{array}$ & $\begin{array}{c}\text { Average Error } \\
\text { (CG3) }\end{array}$ & $\begin{array}{c}\text { Maximum } \\
\text { Error (CG3) }\end{array}$ \\
\hline$T$-linear & 1.9079660 & 8.4207680 & 1.93973600 & 7.1821860 \\
\hline$T$-nonlinear & 1.8602020 & 8.2069160 & 1.90911300 & 6.8886860 \\
\hline$T$-alpha & 0.7539850 & 3.2350280 & 0.79211800 & 3.6257580 \\
\hline$T P 1$ & 0.3346260 & 1.3696930 & 0.47170900 & 2.3380610 \\
\hline$T P 2$ & 0.2442880 & 1.0371920 & 0.29170700 & 1.3782190 \\
\hline$T P 3$ & 0.2092270 & 0.9276220 & 0.72241200 & 2.4142770 \\
\hline$P 1$ & 0.8534630 & 3.1891340 & 0.91875100 & 3.8479100 \\
\hline$P 2$ & 0.7514180 & 2.9953560 & 0.84681100 & 3.6422400 \\
\hline$P 3$ & 0.7514570 & 2.9783900 & 0.82102900 & 3.5877600 \\
\hline$P 4$ & 0.8524760 & 3.1908970 & 0.91211900 & 3.9821170 \\
\hline$P 5$ & 0.7510680 & 2.9737030 & 0.82699600 & 3.5480050 \\
\hline$P 6$ & 0.6039650 & 2.4038430 & 0.71510000 & 3.3758670 \\
\hline$P 7$ & 0.6021840 & 2.3595240 & 0.70264400 & 3.3025030 \\
\hline$P 8$ & 0.7507870 & 2.9217370 & 0.83774900 & 3.6670860 \\
\hline$P 9$ & 0.7519390 & 2.9984000 & 0.83870500 & 3.5670450 \\
\hline$P 10$ & 0.6042490 & 2.3940230 & 0.71061200 & 3.6161170 \\
\hline$P 11$ & 0.6049410 & 2.4063890 & 0.73473200 & 3.4141590 \\
\hline$P 12$ & 0.7543090 & 2.9893530 & 0.83378000 & 3.6477950 \\
\hline$P 13$ & 08493498 & 32149713 & 090250870 & 38633716 \\
\hline$P 14$ & 0.7538413 & 3.0412900 & 0.87024546 & 3.7181478 \\
\hline$P 15$ & 0.7522054 & 3.0022050 & 0.83813760 & 3.5829217 \\
\hline$P 16$ & 0.8504185 & 3.2247367 & 0.91743120 & 4.0959105 \\
\hline & & & & \\
\hline
\end{tabular}

It has been observed from Table 5.12 that the average errors as well as the maximum errors are within acceptable limit i.e., average error $\leq 5 \%$ and the maximum errors $\leq$ $15 \%$. It can be concluded that the model with three-layer perceptrons network is well trained with back propagation as well as conjugate gradient training method. Figure 5.15 shows the testing error plot for T-nonlinear with conjugate gradient training. 


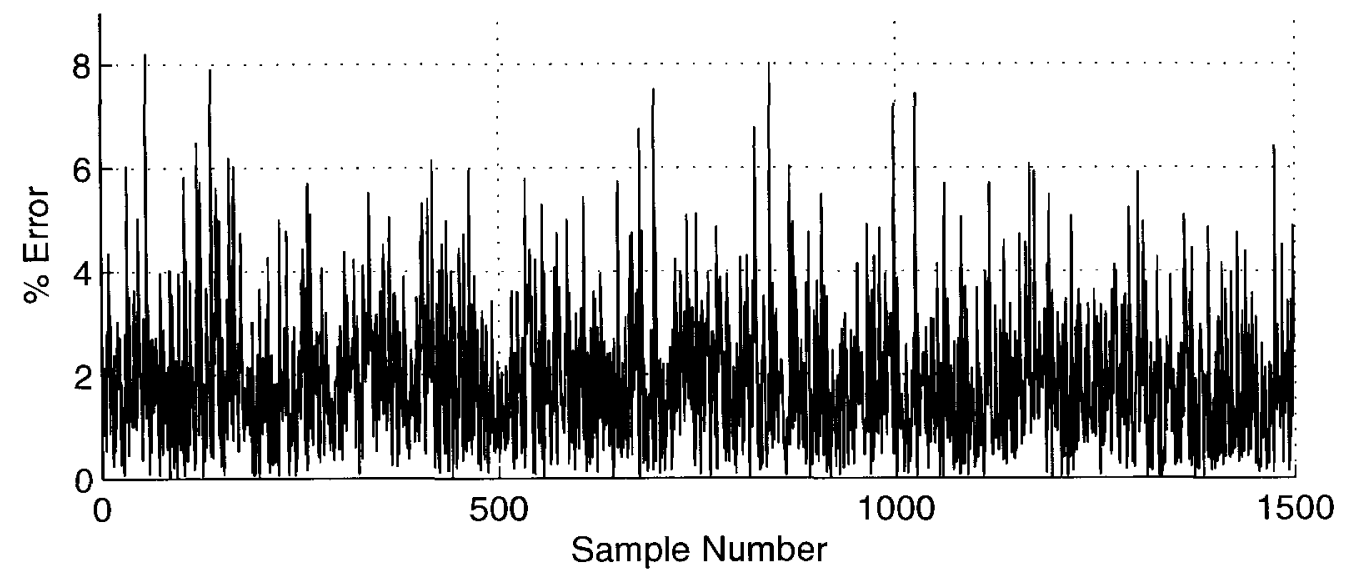

Figure 5.15: Percentage Error of Neural Model for T-nonlinear Using Three-Layer Package Model with Conjugate Gradient Training

Figure 5.16 shows the plot for T-nonlinear vs. sample number and Figure 5.17 shows the output vs. input plot.

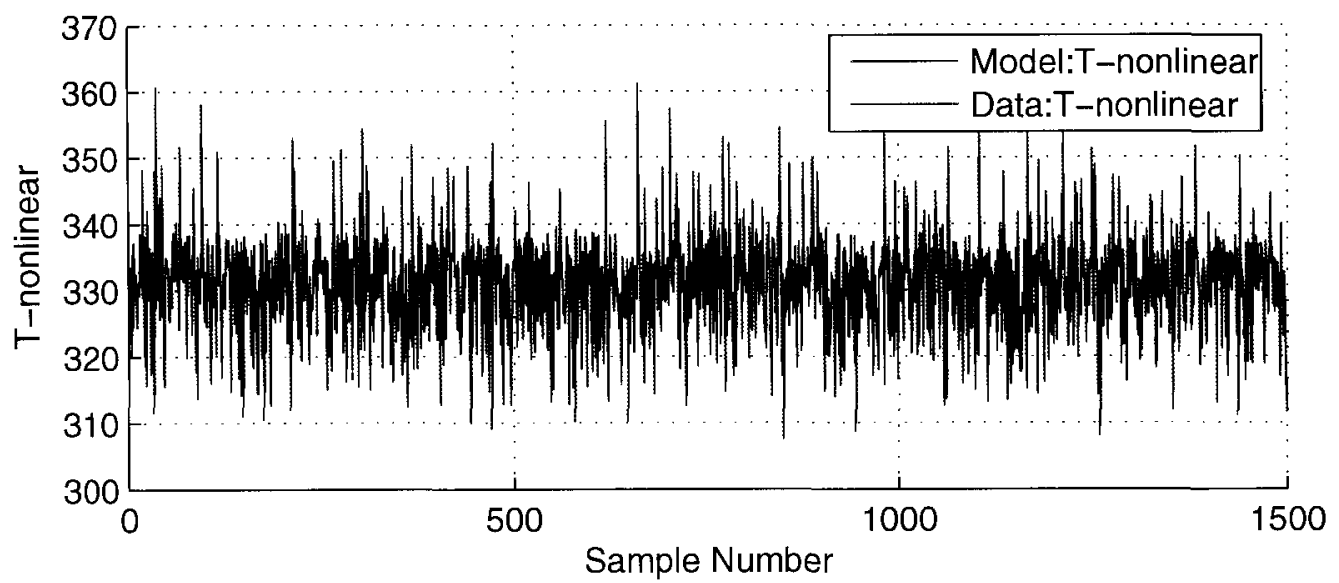

Figure 5.16: Neural Model Output vs. Test Data for T-nonlinear Using Three-Layer Package Model with Back Propagation Training 


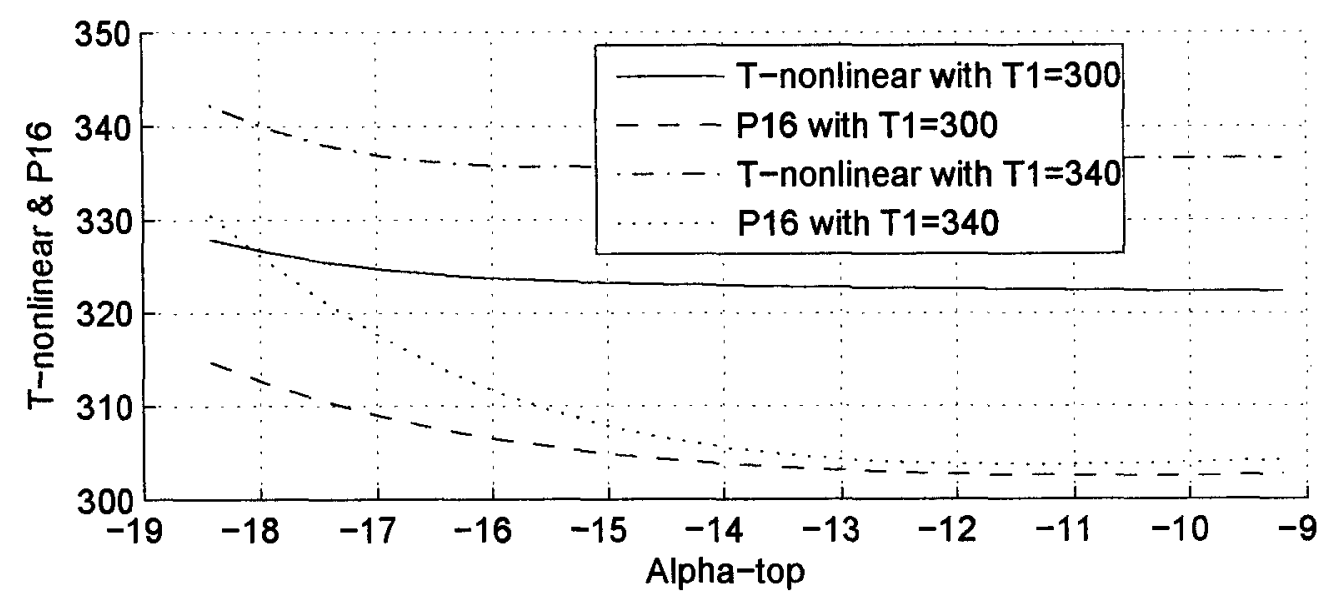

Figure 5.17 (a): T-nonlinear and P16 (Outputs) vs. Alpha-top (Input) w.r.t T1 (Input) for Three-Layer Package Model with Back Propagation Training

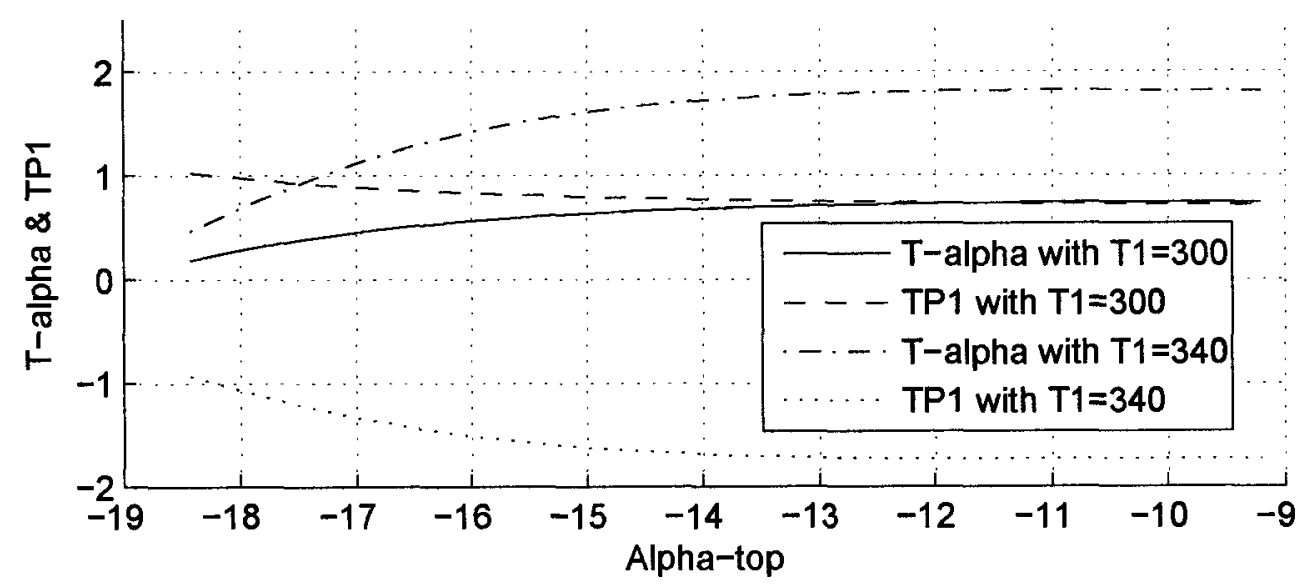

Figure 5.17 (b): T-alpha and TP1 (Outputs) vs. Alpha-top (Input) w.r.t T1 (Input) for Three-Layer Package Model with Back Propagation Training

Figure 5.17 (a) and Figure 5.17 (b) clearly indicate the non-linearity present in the model. The testing errors for four-layer perceptrons network are shown in Table B.3.1 in Appendix B. As mentioned earlier, the four-layer perceptrons network has not been well trained for this model. Consequently, the average and maximum errors are comparatively high for some of the outputs of Package model with four-layer perceptrons. 


\subsection{Summary}

Neural network model have been built for all the three package models. The results obtained from neural network modeling show that the models with three-layer perceptrons have been trained well. But the four-layer perceptrons did not train well. The average testing errors for three-layer perceptrons network are: $0.5 \%$ (four-port $\mathrm{GaN}$ model with $\mathrm{BP}$ ), $0.38 \%$ (four-port GaN model with $\mathrm{CG}$ ), $0.55 \%$ (GaAs model with $\mathrm{BP}$ ), 0.54\% (GaAs model with CG), $0.78 \%$ (Package model with BP) and $0.88 \%$ (Package model with CG). These small errors indicate that neural networks can be utilized to build non-linear thermal models with sufficient accuracy. In the next chapter, the use of submodel to determine the thermal behavior of a detailed model is presented. 


\section{Chapter 6}

\section{Use of Neural Networks Models in Thermal}

\section{Simulation}

This chapter shows how the sub-modeling approach is used to determine the overall thermal behavior of a mixed-model consisting of a detailed model and non-linear submodels based on using a neural network approach. It then presents the comparison between the temperatures of a fully detailed model and a mixed-model for the three package models mentioned earlier.

\subsection{Use of Neural Networks Sub-models in Mixed Model}

As seen from Chapter 5, neural network sub-models have been built by training the models with a set of training data for each model. A fully detailed model can be broken into a base-model and a number of sub-models. To determine the thermal behavior of the mixed-model one needs to solve for the temperatures in the detailed base-model with attached sub-models. This method should be fast, accurate and should be able to represent all the temperatures in the base and the port temperatures and temperatures of interest of the sub-model. The set of equations which describe this problem are non-linear 
due to the non-linear neural network sub-models. The solution of this set of equations can be obtained in an iterative way by using the MATLAB/C code - representing the inputoutput relationships of the thermal sub-model - that is possible to generate using Neuromodeler. Maximum temperature of each sub-model as well as temperatures at different points of interest can be determined through this iteration process. It is worthwhile to mention that the training and testing errors were less when the neural network had been based on three-layer perceptrons, as observed in Chapter 5 . Consequently, the MATLAB/C code obtained from three-layer perceptrons network has been utilized in this chapter for calculating the temperatures of the sub-model.

Various issues are involved in calculating the temperatures in this iterative way. Convergence is one of the major issues. The iteration process continues until the convergence criterion is met. In this thesis, the convergence is chosen as $1 \mathrm{e}^{-5} \mathrm{~K}$. Another concern is the initial port temperatures used at the start of the iteration. In order to determine the port heat-flows and output temperatures (T-max and other temperatures of interest) the input port temperatures (initial guess for the iteration) should be chosen such that the iteration converges. These initial port temperatures can be easily selected in two obvious ways: these temperatures can either be taken as $300 \mathrm{~K}$ or they can be obtained from a solution of the linear mixed-model comprised of the base-model (which is linear) and linear sub-models generated using Atar. It has been observed that if the initial guess for port temperature is selected as $300 \mathrm{~K}$, it does not provide consistent convergence. Consequently, the initial guess for port temperature for the iteration has been taken from linear solution, which provides a much closer approximation to the actual port 
temperatures of the non-linear problem. Another issue that relates to the convergence of the solution is the calculation of the "new" port temperatures at the end of each iteration. These temperatures can easily be extracted from the new base-model temperatures; however, it has been found that for robust convergence it was needed to average them with the previous iterations values. The formula used to do this is:

$$
T_{p}=\frac{T_{p_{\text {new }}}+\text { alpha } \times T_{p_{\text {old }}}}{1+\text { alpha }}
$$

where, alpha determines the degree of averaging. For alpha $=0$ we have simple iteration and for alpha greater than one we have a specified degree of averaging. A trade-off will exist between number of iterations to convergence and robustness of the algorithm.

The pseudo code for determining the output temperatures in the above-mentioned iterative process is provided at the end of this section. After calculating the maximum temperatures and temperatures at different points of interest, errors have been calculated using Equation (6.2) for temperatures of each sub-model with respect to the temperatures of the detailed model.

$$
\text { Error }=\frac{\left|T_{\text {det }}-T_{\text {sub }}\right|}{T_{\text {det }}-300}
$$

where, $T_{\text {det }}$ is the temperature at a point of interest in the fully detailed model and $T_{s u b}$ is the corresponding temperature of the sub-model. 


\section{Pseudo Code:}

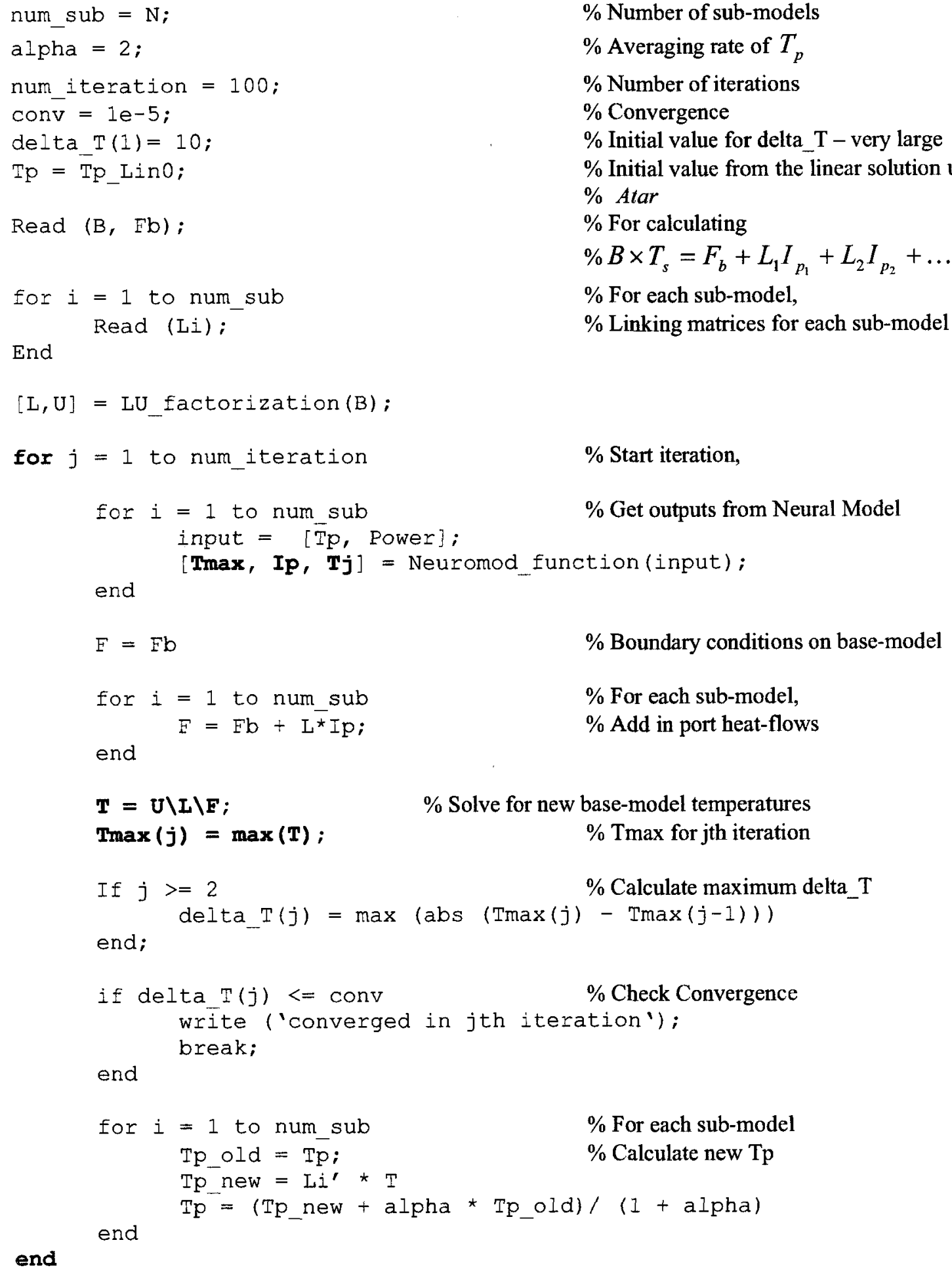




\subsection{Full Models Obtained from Neural Networks Sub-models}

This section presents the temperature prediction at different points of interest for submodels as well as detailed model for the three models mentioned earlier. It then calculates the errors for temperatures of the sub-models with respect to that of the detailed model.

\subsubsection{GaN Based Power Package Amplifier}

A detailed model of a GaN amplifier has been represented by eight sub-models. Figure 6.1 (a) and Figure 6.1 (b) show the layouts of the GaN sub-model and the amplifier. Figure 6.2 shows a view of the fully detailed Atar model of the amplifier. Details of the GaN sub-model attachment and a fully detailed model can be seen in Figure 6.3. The GaN amplifier had a fixed 300K BC applied to the bottom of the model.

Table 6.1 and Table 6.2 show the temperatures at different points of interest for a detailed model and two sub-models and corresponding errors for GaN model with four-port configuration and five-port configuration respectively. The output temperatures for this model have been calculated at the point where the maximum temperature is reached and also at three points of each of the fingers of $\mathrm{GaN}$, i.e., a total of eighteen points. 


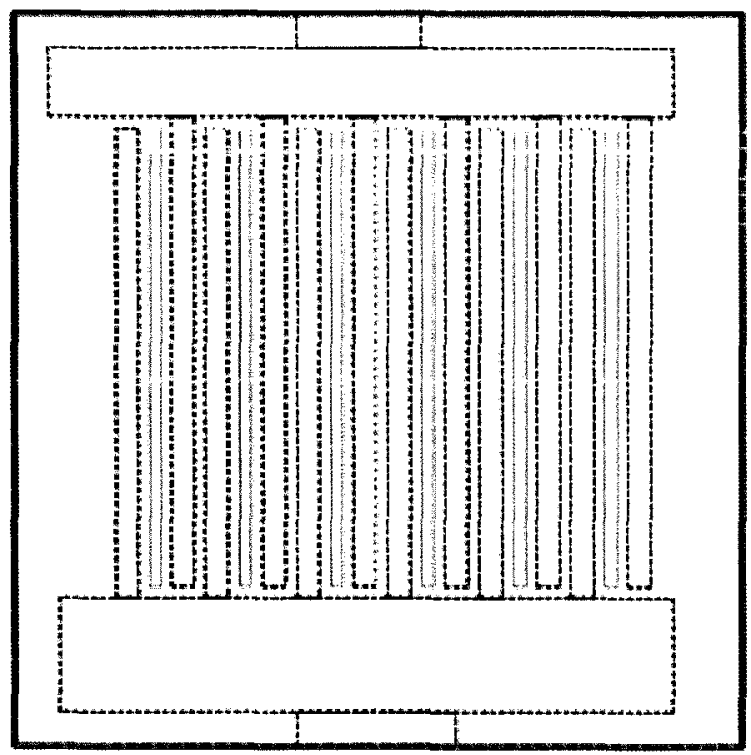

(a)

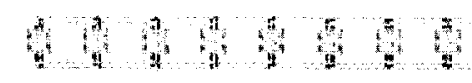

(b)

Figure 6.1 (a): GaN Sub-model Layout Figure 6.1 (b): GaN Mixed-model Layout

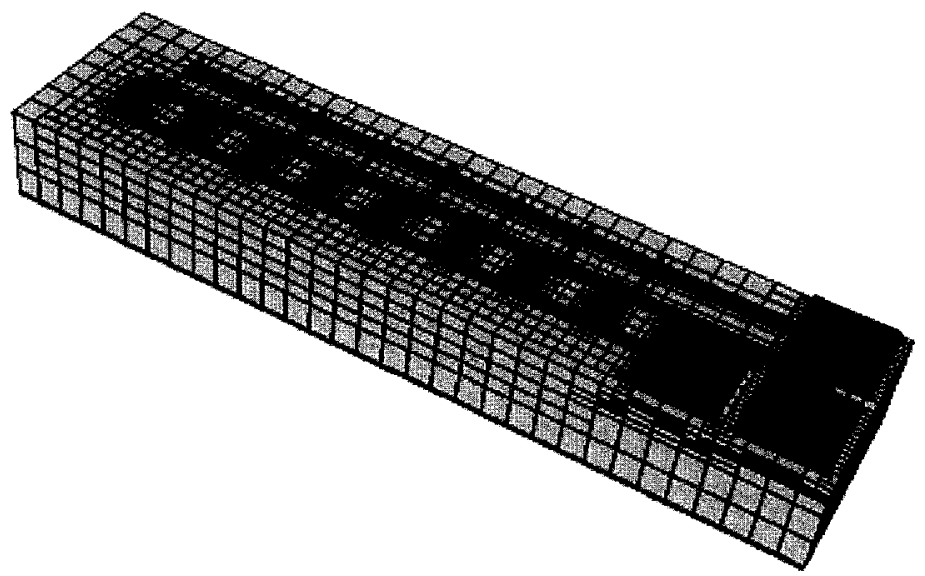

Figure 6.2: Detailed GaN Model 


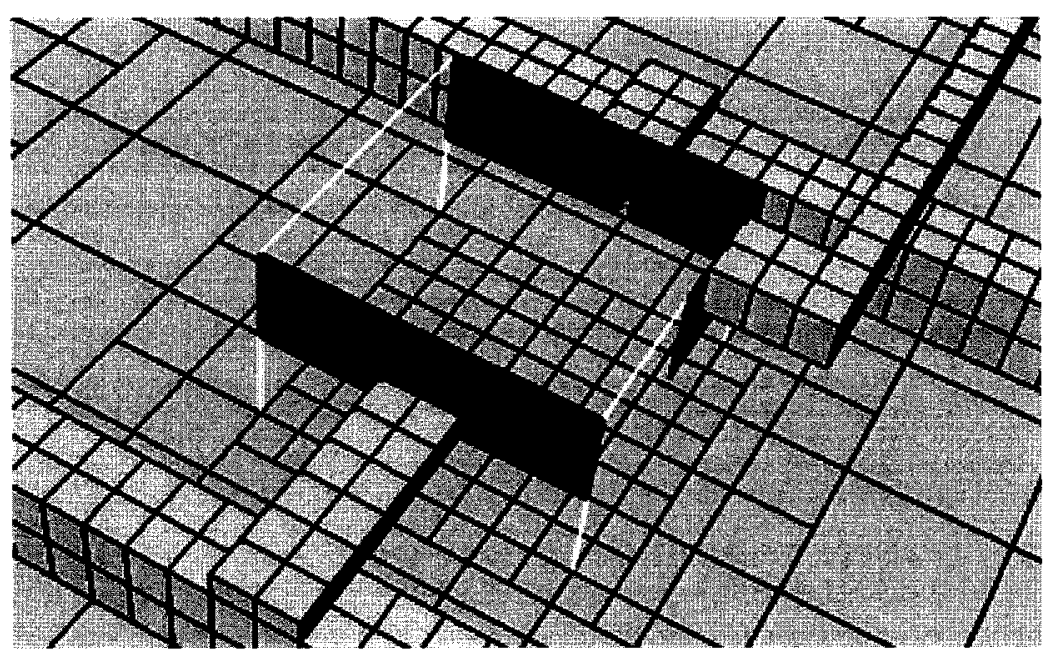

Figure 6.3 (a): Details of GaN Sub-model Placement

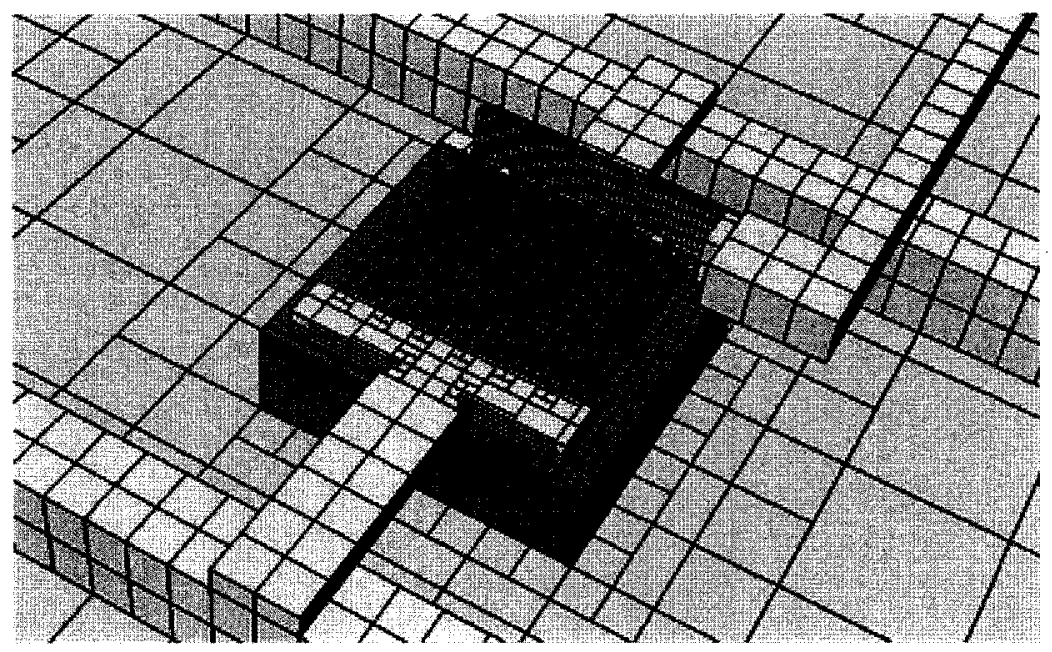

Figure 6.3 (b): GaN Full-model Showing GaN Amplifier Detail 


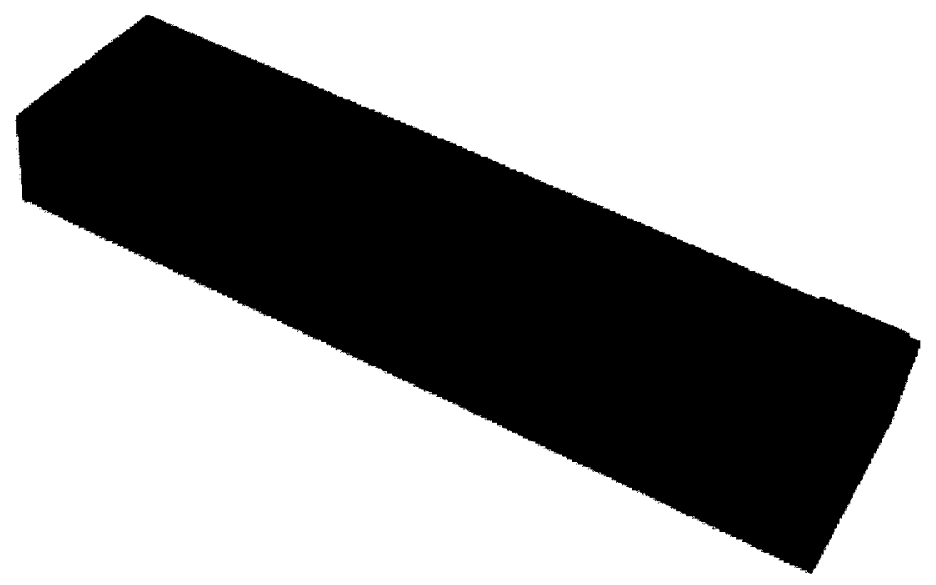

(a)
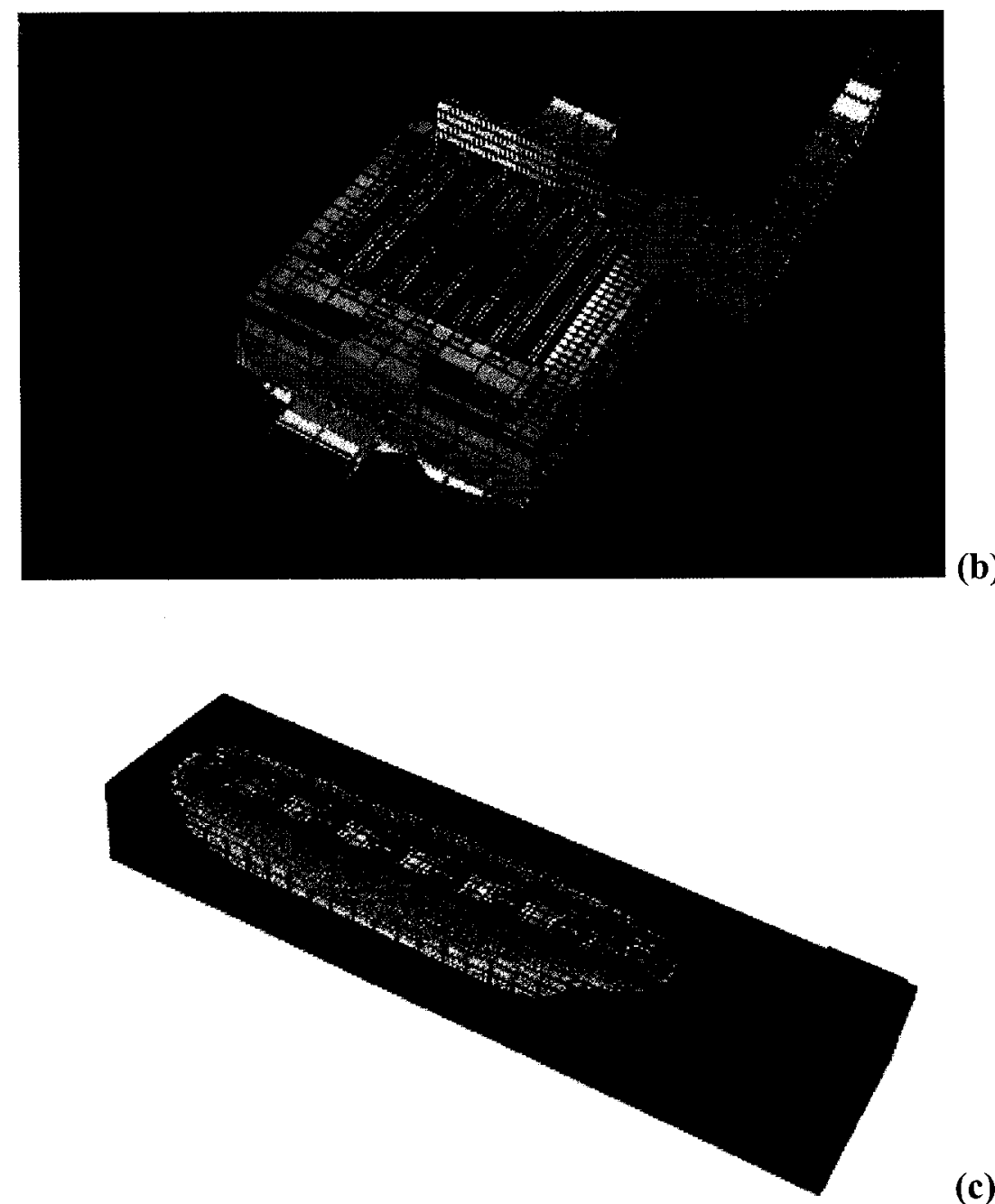

(c)

Figure 6.4: (a) Temperature of Detailed GaN Model (b) Details View of Temperature of Fully Detailed GaN Model (c) Temperature of GaN Base-model Using Sub-model 
Figure 6.4 (a) shows the temperature profile of a detailed GaN model. Figure 6.4 (b) shows the same model in detail and presents one of the individual power cells. It has been observed that the temperature is higher (appearing as red) in the centre, which of course is where the power is created. Figure 6.4 (c) shows the temperature of the GaN basemodel obtained using a mixed-model with attached sub-models. It has been observed from this figure that the model achieves the maximum temperature (appearing as red) in the centre of the sub-model.

Table 6.1: Output Temperatures for Detailed and Sub-models and Corresponding Errors of GaN Model with Four-Port Configuration for Back-side BC

\begin{tabular}{|c|c|c|c|c|c|c|}
\hline & \multicolumn{3}{|c|}{ Model-1 } & \multicolumn{3}{c|}{ Model-2 } \\
\hline Output & T full & T sub & Error & T full & T sub & Error \\
\hline & $\mathbf{( K )}$ & $\mathbf{( K )}$ & & $\mathbf{( K )}$ & $\mathbf{( K )}$ & \\
\hline$I-\max$ & 398.317029 & 399.7594 & 0.014671 & 400.581279 & 402.3671 & 0.017755 \\
\hline$I I-1$ & 372.991361 & 371.4121 & 0.021636 & 375.434600 & 373.5863 & 0.024502 \\
\hline$I 1-2$ & 377.110713 & 378.4875 & 0.017855 & 379.590885 & 380.7268 & 0.014272 \\
\hline$I I-3$ & 373.440987 & 372.4964 & 0.012862 & 375.848452 & 374.7434 & 0.014569 \\
\hline$I I-4$ & 373.227389 & 374.8620 & 0.022322 & 375.582251 & 377.1359 & 0.020556 \\
\hline$I I-5$ & 378.850890 & 383.4079 & 0.057793 & 381.250747 & 385.7992 & 0.055980 \\
\hline$I 1-6$ & 374.102257 & 376.2137 & 0.028494 & 376.413743 & 378.4872 & 0.027135 \\
\hline$I 2-1$ & 378.764022 & 375.7625 & 0.038108 & 377.167597 & 378.0198 & 0.011044 \\
\hline$I 2-2$ & 384.936679 & 385.2393 & 0.003563 & 383.497458 & 387.6618 & 0.049874 \\
\hline$I 2-3$ & 379.409432 & 377.1021 & 0.029056 & 378.032018 & 379.2414 & 0.015499 \\
\hline$I 2-4$ & 375.397708 & 376.7933 & 0.018510 & 377.572127 & 379.0214 & 0.018683 \\
\hline$I 2-5$ & 381.747531 & 383.3897 & 0.020088 & 383.967451 & 385.7068 & 0.020715 \\
\hline$I 2-6$ & 376.392262 & 377.1110 & 0.009409 & 378.519608 & 379.2982 & 0.009916 \\
\hline$I 3-1$ & 374.767556 & 375.6170 & 0.011361 & 380.633531 & 377.8369 & 0.034683 \\
\hline$I 3-2$ & 380.641508 & 383.8801 & 0.040160 & 386.374110 & 386.1167 & 0.002980 \\
\hline$I 3-3$ & 375.686582 & 377.1087 & 0.018790 & 381.246595 & 379.4082 & 0.022627 \\
\hline$I 3-4$ & 375.628724 & 373.6995 & 0.025509 & 377.698770 & 375.8596 & 0.023671 \\
\hline$I 3-5$ & 380.179454 & 377.7390 & 0.030437 & 382.265884 & 380.0177 & 0.027328 \\
\hline$I 3-6$ & 375.892896 & 373.2756 & 0.034487 & 377.919380 & 375.3908 & 0.032451 \\
\hline
\end{tabular}


Table 6.2: Output Temperatures for Detailed and Sub-models and Corresponding Errors of GaN Model with Five-Port Configuration for Back-side BC

\begin{tabular}{|c|c|c|c|c|c|c|}
\hline & \multicolumn{3}{|c|}{ Model-1 } & \multicolumn{3}{c|}{ Model-2 } \\
\hline Output & T full & T sub & Error & T_full & T_sub & Error \\
\hline & $\mathbf{( K )}$ & $\mathbf{( K )}$ & & $\mathbf{( K )}$ & $(\mathbf{K})$ & \\
\hline$I-\max$ & 398.317029 & 402.2699 & 0.040205 & 400.581279 & 404.8189 & 0.042131 \\
\hline$I 1-1$ & 372.991361 & 371.4787 & 0.020724 & 375.434600 & 374.2894 & 0.015181 \\
\hline$I 1-2$ & 377.110713 & 378.6016 & 0.019334 & 379.590885 & 381.4354 & 0.023175 \\
\hline$I 1-3$ & 373.440987 & 373.0715 & 0.005031 & 375.848452 & 376.1865 & 0.004457 \\
\hline$I 1-4$ & 373.227389 & 376.0694 & 0.038811 & 375.582251 & 379.0280 & 0.045589 \\
\hline$I 1-5$ & 378.850890 & 384.0103 & 0.065432 & 381.250747 & 387.0473 & 0.071342 \\
\hline$I 1-6$ & 374.102257 & 377.1412 & 0.041010 & 376.413743 & 380.0923 & 0.048140 \\
\hline$I 2-1$ & 378.764022 & 376.9989 & 0.022410 & 377.167597 & 380.0065 & 0.036789 \\
\hline$I 2-2$ & 384.936679 & 385.8146 & 0.010336 & 383.497458 & 388.7163 & 0.062503 \\
\hline$I 2-3$ & 379.409432 & 378.5420 & 0.010924 & 378.032018 & 381.4978 & 0.044415 \\
\hline$I 2-4$ & 375.397708 & 377.8957 & 0.033131 & 377.572127 & 380.7261 & 0.040659 \\
\hline$I 2-5$ & 381.747531 & 384.3663 & 0.032035 & 383.967451 & 387.1374 & 0.037752 \\
\hline$I 2-6$ & 376.392262 & 377.6136 & 0.015988 & 378.519608 & 380.3876 & 0.023790 \\
\hline$I 3-1$ & 374.767556 & 376.9795 & 0.029584 & 380.633531 & 379.8680 & 0.009494 \\
\hline$I 3-2$ & 380.641508 & 384.8099 & 0.051690 & 386.374110 & 387.5195 & 0.013261 \\
\hline$I 3-3$ & 375.686582 & 378.2054 & 0.033280 & 381.246595 & 380.9507 & 0.003642 \\
\hline$I 3-4$ & 375.628724 & 375.3584 & 0.003574 & 377.698770 & 378.1030 & 0.005203 \\
\hline$I 3-5$ & 380.179454 & 379.3287 & 0.010611 & 382.265884 & 382.0114 & 0.003093 \\
\hline$I 3-6$ & 375.892896 & 375.0958 & 0.010503 & 377.919380 & 377.8878 & 0.000405 \\
\hline
\end{tabular}

The results for other sub-models are shown in Table C.1.1 to Table C.1.3 (for four-port configuration) and in Table C.1.4 to Table C.1.6 (for five-port configuration) of Appendix C. It can be observed that the errors are small $(\leq 7 \%$ and $\leq 8 \%$ for four-port and five-port configuration respectively) when the detailed model is represented by a submodel with four-port and five-port configurations.

Figure 6.5 (a) and Figure 6.5 (b) show the temperature error of GaN sub-model with fourport configuration and Figure 6.5 (c) shows the temperature error of the same model with five-port configuration. It has been observed from these figures that the temperature error is higher (appearing as red) in the centre of the sub-model for four-port configuration and 
for five-port configuration temperature error is higher (appearing as red) in the centre and also in one of the metal ports. It can be observed that the temperature error for the majority of the structure is very small and the error is concentrated at the ports where a constant temperature is imposed on a region of the base-model.

Table 6.3 shows the temperature rise and maximum error for linear and non-linear substrate simulations.

Table 6.3: Temperature Rise and Maximum Error for Linear and Non-linear Substrate of GaN Model

\begin{tabular}{|c|c|c|c|}
\hline Port & Substrate & Absolute Error (K) & Maximum Error (\%) \\
\hline 4-port & linear & 3.44 & 7.50 \\
\hline 4-port & non-linear & 4.09 & 8.50 \\
\hline 5-port & linear & 6.50 & 13.8 \\
\hline 5-port & non-linear & 6.40 & 13.3 \\
\hline
\end{tabular}

It has been observed from Table 6.3 that when a mixed-model is used with a particular port configuration introduced for a sub-model, there is a temperature error created by the imposition of a uniform temperature over a port. Table 6.3 shows that for a four-port configuration, the temperature rise and error are more for non-linear sub-model than that of linear sub-model. For five-port configuration, the error is actually less for non-linear sub-model than that of linear sub-model. These temperature errors are within satisfactory limit. The errors presented here indicate that the primary source of error is not due to the use of a non-linear sub-model but just due to the use of sub-models and the portconfiguration selected. 


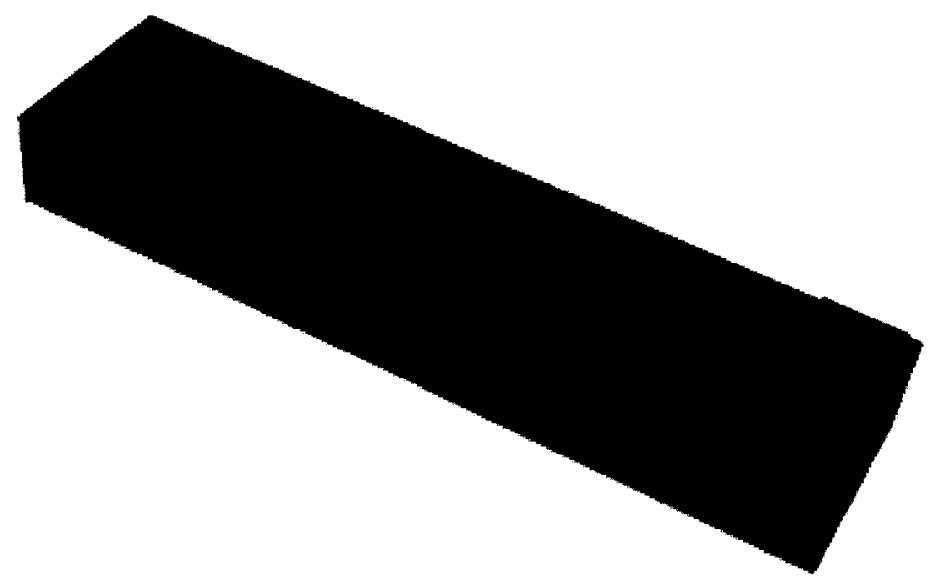

(a)

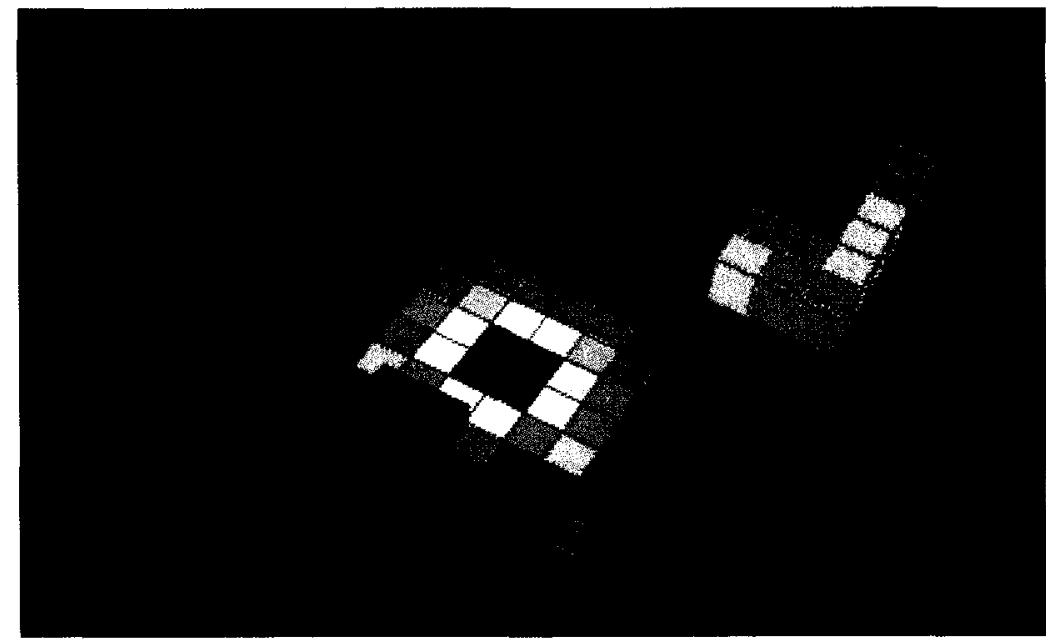

(b)

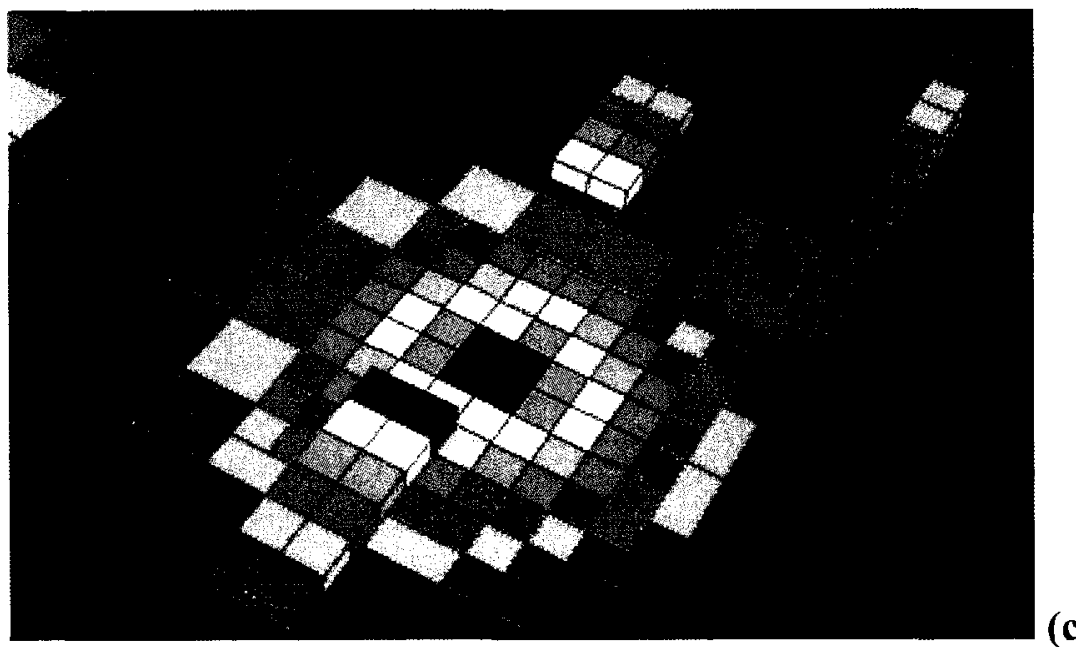

(c)

Figure 6.5: (a) Temperature Error of GaN Sub-model with Four-Port Configuration (b) Detailed View of Temperature Error for Four-Port Configuration (c) Temperature Error of GaN Sub-model with Five-Port Configuration 


\subsubsection{GaAs Based Power Amplifier}

The mixed-model of a GaAs power amplifier has been built using ten sub-models with five-port configuration. A detailed thermal model of the GaAs power amplifier die with a five-section cascade gain cell structure is shown in Figure 6.6 (a). A fully detailed model of the amplifier is shown in Figure $6.6(\mathrm{~b})$.

The output temperatures have been calculated at the point where the maximum temperature of the model is reached and also at three different points at each of the four fingers, i.e., a total of twelve points. Table 6.4 shows the temperatures at different points of interest for both detailed and two sub-models and corresponding errors for GaAs model with five-port configuration with a fixed $300 \mathrm{~K} \mathrm{BC}$ applied to the back-side of the model.

Table 6.4: Output Temperatures for Detailed and Sub-models and Corresponding Errors of GaAs Model with Back-side BC

\begin{tabular}{|c|c|c|c|c|c|c|}
\hline & \multicolumn{3}{|c|}{ Model-1 } & \multicolumn{3}{c|}{ Model-2 } \\
\hline Output & T_full & T_sub & Error & T full & T sub & Error \\
\hline & $\mathbf{( K )}$ & $\mathbf{( K )}$ & & $(\mathbf{K})$ & $(\mathbf{K})$ & \\
\hline$I-$ max & 460.618249 & 461.6152 & 0.006207 & 466.532133 & 466.9409 & 0.002455 \\
\hline$I 1-1$ & 397.865100 & 395.0147 & 0.029126 & 399.651200 & 398.5926 & 0.010623 \\
\hline$I 1-2$ & 459.851800 & 459.1584 & 0.004338 & 466.111600 & 463.6721 & 0.014686 \\
\hline$I 1-3$ & 448.709700 & 446.8892 & 0.012242 & 451.209200 & 451.1796 & 0.000196 \\
\hline$I 2-1$ & 398.886600 & 397.4286 & 0.014744 & 401.503600 & 401.0870 & 0.004104 \\
\hline$I 2-2$ & 464.764800 & 462.0909 & 0.016229 & 467.108200 & 466.4999 & 0.003640 \\
\hline$I 2-3$ & 451.816500 & 449.5302 & 0.015060 & 454.499600 & 453.9034 & 0.003859 \\
\hline$I 3-1$ & 399.190300 & 397.8209 & 0.013806 & 403.244600 & 401.4333 & 0.017544 \\
\hline$I 3-2$ & 463.214700 & 463.1592 & 0.000340 & 468.350500 & 467.5339 & 0.004851 \\
\hline$I 3-3$ & 452.749400 & 450.2851 & 0.016133 & 455.253400 & 454.6569 & 0.003842 \\
\hline$I 4-1$ & 395.936300 & 394.6022 & 0.013906 & 398.306300 & 398.2605 & 0.000466 \\
\hline$I 4-2$ & 459.293100 & 457.4468 & 0.011591 & 464.134900 & 461.8946 & 0.013649 \\
\hline$I 4-3$ & 448.735600 & 446.3598 & 0.015973 & 451.905500 & 450.5702 & 0.008790 \\
\hline
\end{tabular}


The results for other sub-models are shown in Table C.2.1 to Table C.2.4 of Appendix C. It can be observed that the errors are small (within 3\%) when the mixed-model is represented by the sub-models with five-port configuration.

Figure 6.7 (a) shows the temperature of a GaAs model for ten sub-models. It has been observed from the figure that the temperature is higher (appearing as red) in the centre of the sub-model. Figure 6.7 (b) shows the detailed view of temperature in the sub-model. Figure 6.7 (c) shows the temperature error (appearing as red) in the substrate of the GaAs model. The error is clearly concentrated around the regions where the ports of the submodels are attached to the base-model.

Table 6.5 shows the temperature rise and maximum error for linear and non-linear substrate.

Table 6.5: Temperature Rise and Maximum Error for Linear and Non-linear Substrate of GaAs Model

\begin{tabular}{|c|c|c|}
\hline Substrate & Absolute Error (K) & Maximum Error (\%) \\
\hline Linear & 2.06 & 7.20 \\
\hline Non-linear & 3.06 & 10.33 \\
\hline
\end{tabular}

It has been observed from Table 6.5 that the primary source of error in temperature is due to the port-configuration and port-selection of the sub-model. The error for a non-linear sub-model is only slightly higher than that of the linear sub-model indicating the primary source of error is due to the use of sub-models. 


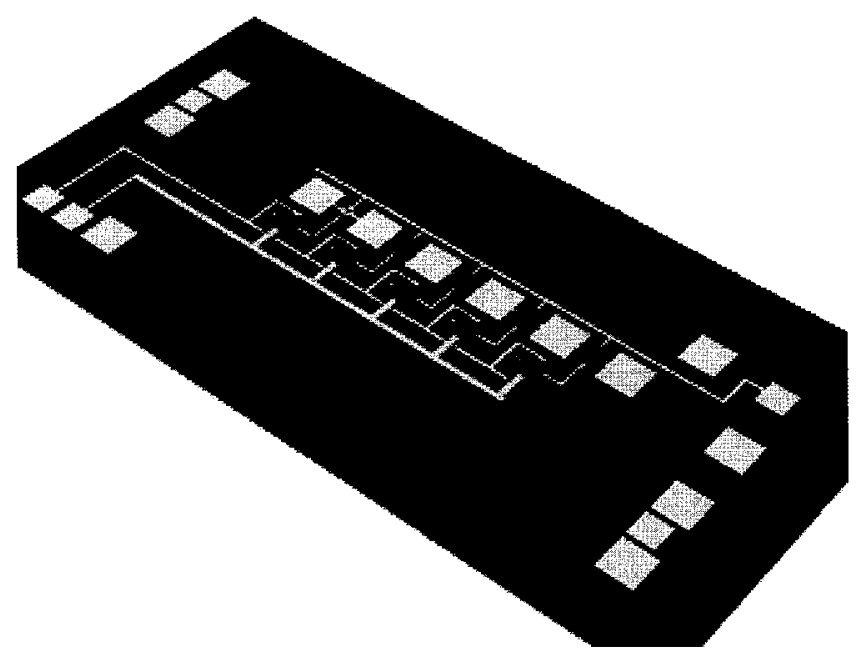

Figure 6.6 (a): GaAs Model

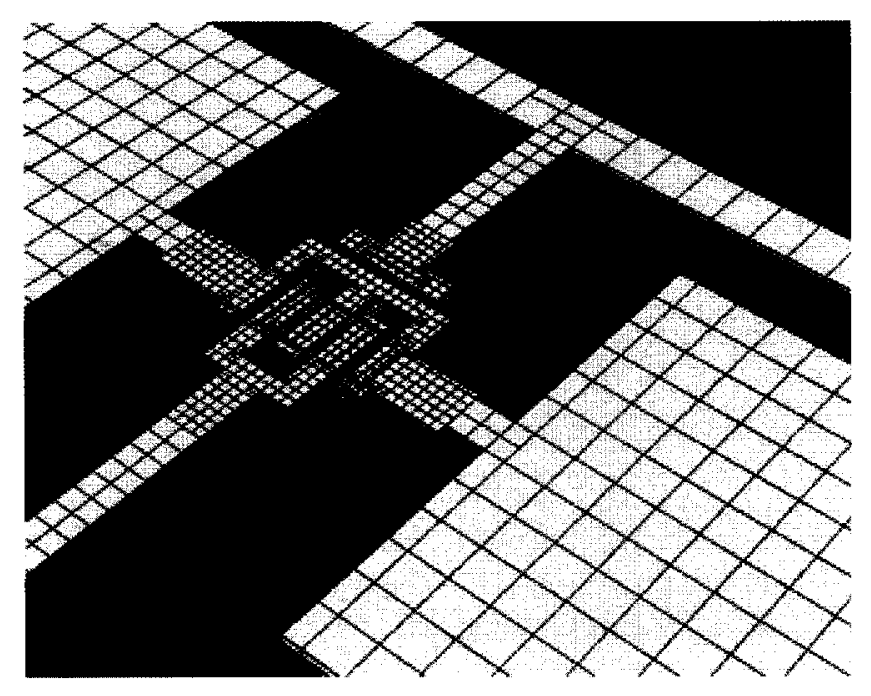

Figure 6.6 (b): Detail GaAs Model 


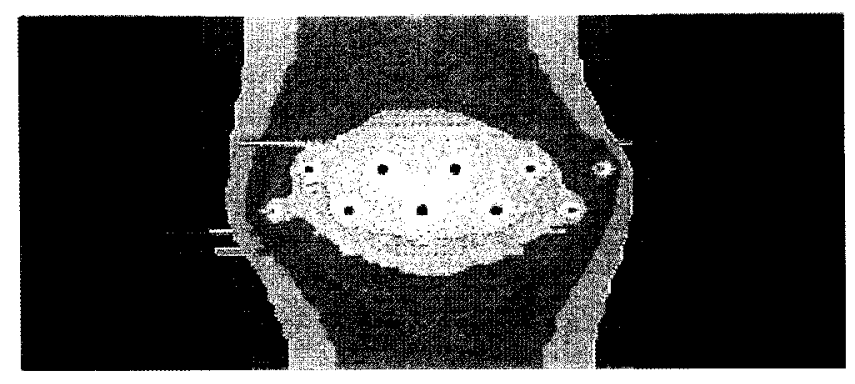

(a)
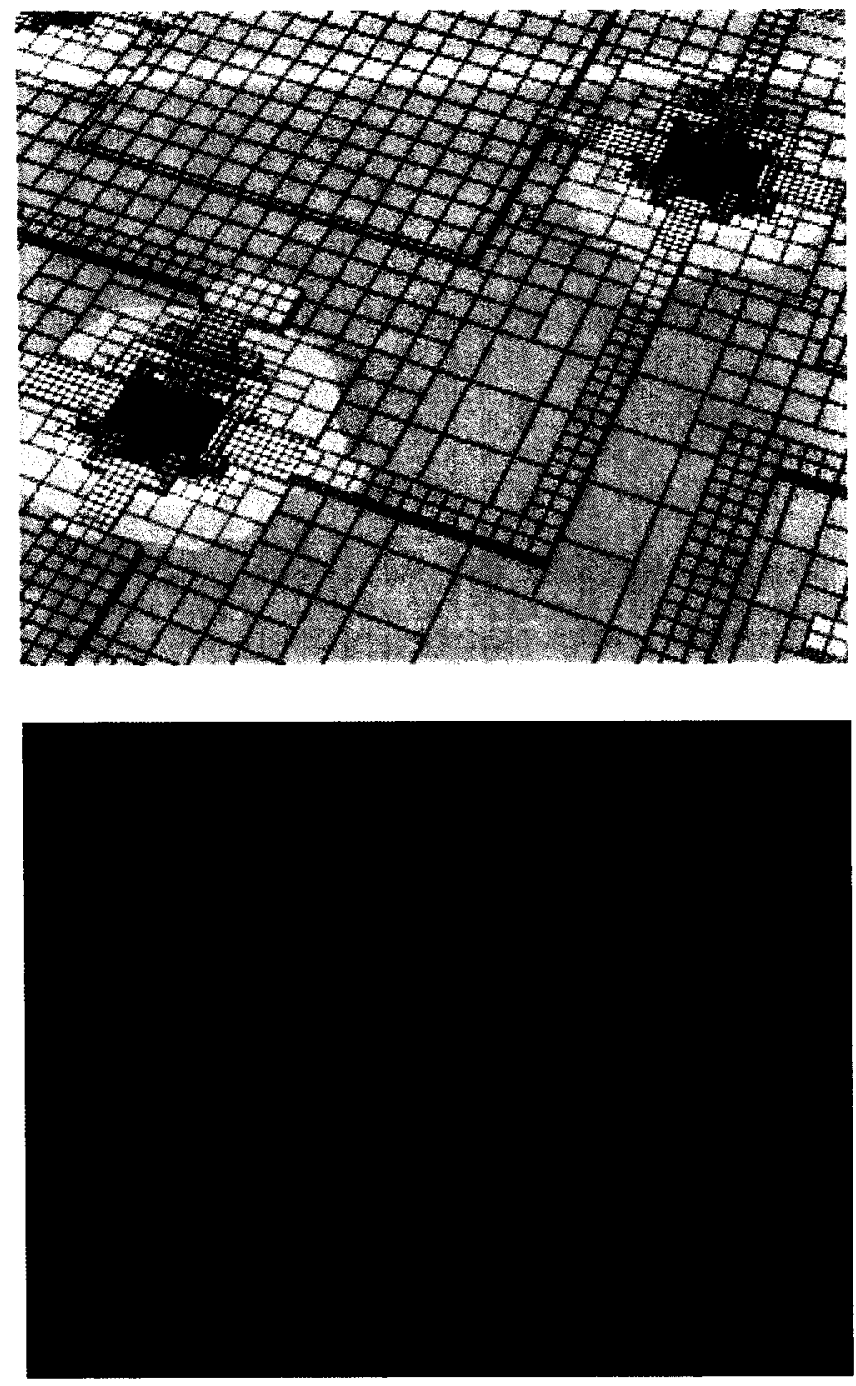

(c)

Figure 6.7: (a) Top View of Temperature of GaAs Model (b) Detail Temperature of GaAs Model (c) Temperature Error in the Substrate of GaAs Model 


\subsubsection{Package Model}

To test the use of the package sub-model two mixed-models have been built. Two models, one consists of a substrate with a single package on it and the second one with four packages, have been built. In each model, temperatures have been calculated for the package model at the point where maximum temperature is reached and also at sixteen different points of interest for both detailed and sub-model. Table 6.6 shows the temperatures for a single package model for both detailed and sub-model and corresponding errors for right-side $\mathrm{BC}$ and $\alpha=10^{-8} \mathrm{~W} / \mu \mathrm{m}^{2} \mathrm{~K}$.

Table 6.6: Output Temperatures for Detailed and Sub-models and Corresponding Errors for Single Package Model with Right-side BC and $\alpha=10^{-8} \mathrm{~W} / \mu \mathrm{m}^{2} \mathrm{~K}$

\begin{tabular}{|c|c|c|c|}
\hline Output & T_full & T_sub & Error \\
\hline & (K) & (K) & \\
\hline$T-m a x$ & 346.941185 & 346.5886 & 0.007511 \\
\hline$P 1$ & 323.361377 & 322.2910 & 0.045818 \\
\hline$P 2$ & 327.192993 & 325.7831 & 0.051848 \\
\hline$P 3$ & 327.193163 & 325.7805 & 0.051949 \\
\hline$P 4$ & 323.361791 & 322.2934 & 0.045732 \\
\hline$P 5$ & 327.271009 & 325.7765 & 0.054802 \\
\hline$P 6$ & 336.200138 & 335.5783 & 0.017178 \\
\hline$P 7$ & 336.200296 & 335.5648 & 0.017555 \\
\hline$P 8$ & 327.271402 & 325.7682 & 0.055120 \\
\hline$P 9$ & 327.271182 & 325.7785 & 0.054735 \\
\hline$P 10$ & 336.200304 & 335.5803 & 0.017127 \\
\hline$P 11$ & 336.200468 & 335.5824 & 0.017073 \\
\hline$P 12$ & 327.271574 & 325.7693 & 0.055086 \\
\hline$P 13$ & 323.361809 & 322.2992 & 0.045485 \\
\hline$P 14$ & 327.193410 & 325.7819 & 0.051906 \\
\hline$P 15$ & 327.193574 & 325.7816 & 0.051923 \\
\hline$P 16$ & 323.362224 & 322.2977 & 0.045566 \\
\hline
\end{tabular}

It has been observed from table 6.6 that the errors are small (within 6\%) when a detailed model is represented with a single package sub-model with three-port configuration. 
Figure 6.8 (a) and Figure 6.8 (b) show the substrate temperature of a detailed package model and mixed-model. It has been observed from the figures that the maximum temperature is reached at the centre (appearing as red) of the sub-model attachment.

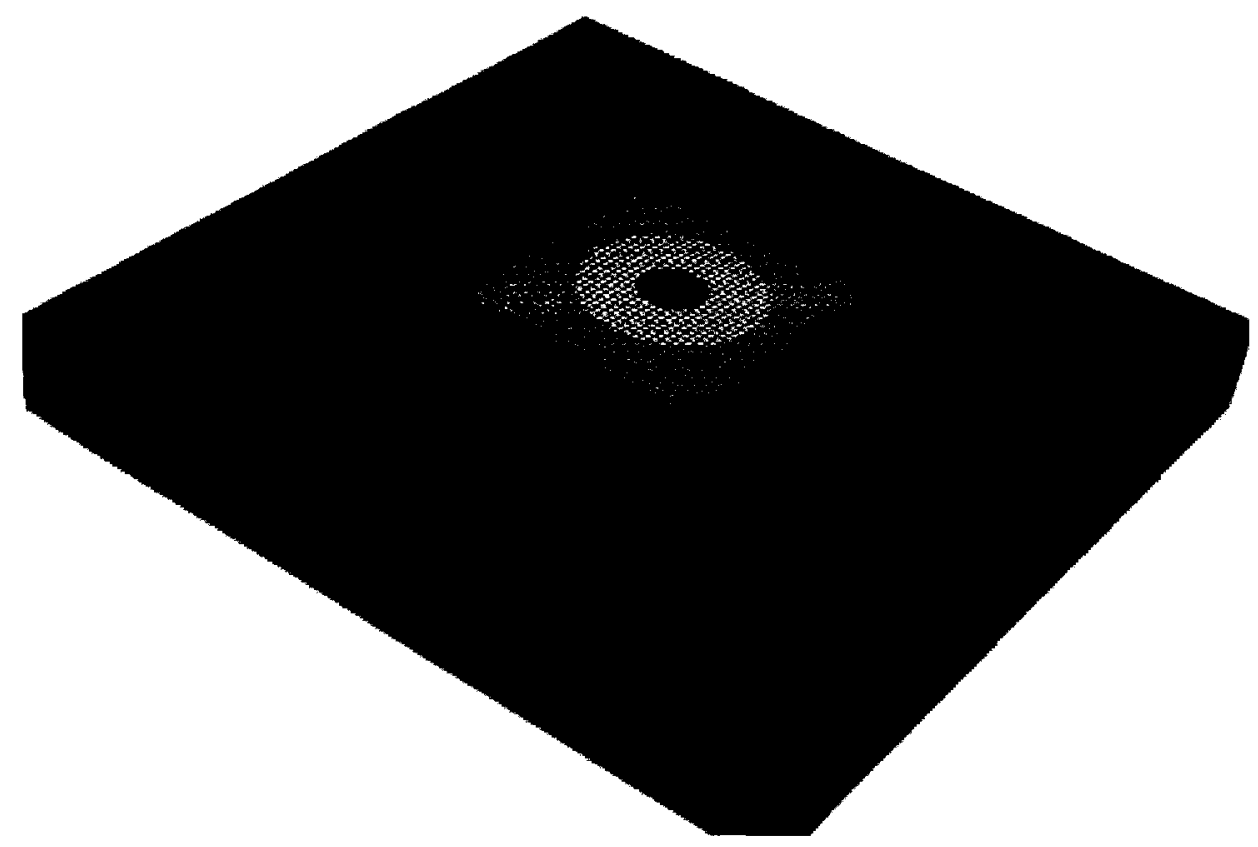

Figure 6.8 (a): Temperatures for Substrate of Package Model Using Detailed Model

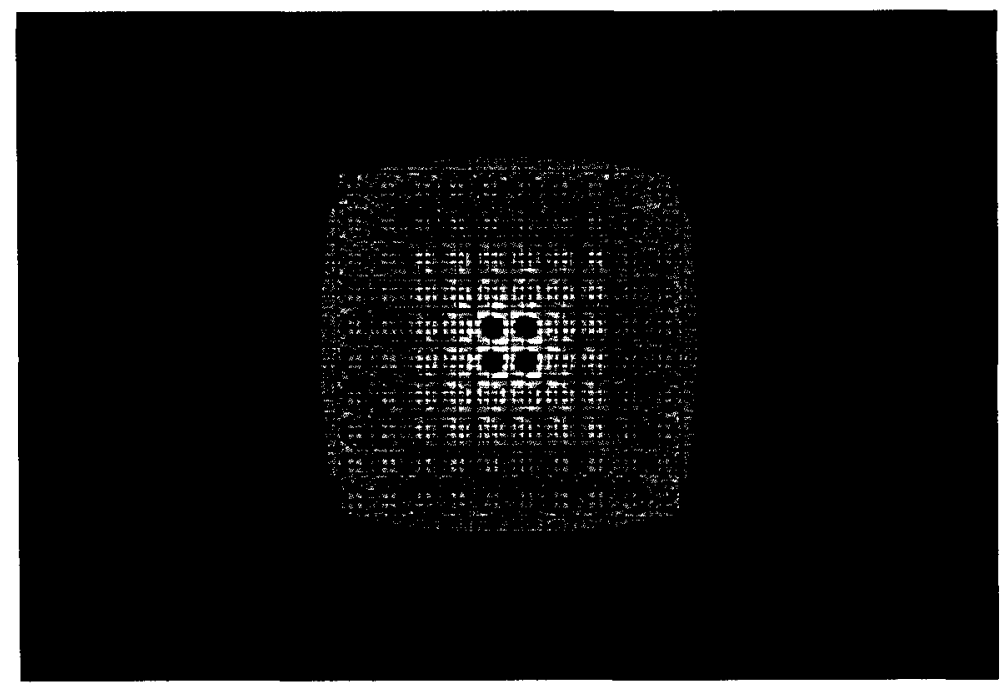

Figure 6.8 (b): Temperatures for Substrate of Package Model Using Sub-model 
Table 6.7 and Table 6.8 show the temperatures for mixed-model with four attached packages for both detailed and sub-models (results for two sub-models are shown here) and corresponding errors for right-side $\mathrm{BC}$ and back-side $\mathrm{BC}$ respectively. The results for other sub-models are shown in Table C.3.1 (for right-side BC) and Table C.3.2 (for backside $\mathrm{BC}$ ) of Appendix C.

Table 6.7: Output Temperatures for Detailed and Two Sub-models and Corresponding Errors of Four-Package Model with Right-side $\mathrm{BC}$ and $\alpha=10^{-8}$ $\mathbf{W} / \mu \mathbf{m}^{2} \mathbf{K}$

\begin{tabular}{|c|c|c|c|c|c|c|}
\hline & \multicolumn{3}{|c|}{ Model-1 } & \multicolumn{3}{c|}{ Model -2 } \\
\hline Output & T full & T_sub & Error & T full & T_sub & Error \\
\hline & $(\mathbf{K})$ & $(\mathbf{K})$ & & $(\mathbf{K})$ & $(\mathbf{K})$ & \\
\hline$T-m a x$ & 355.732599 & 350.8385 & 0.087814 & 355.726242 & 350.7732 & 0.088882 \\
\hline$P 1$ & 337.979876 & 334.6466 & 0.087764 & 338.033587 & 334.6149 & 0.089886 \\
\hline$P 2$ & 340.789132 & 337.1676 & 0.088787 & 340.805374 & 337.1306 & 0.090056 \\
\hline$P 3$ & 340.810582 & 337.1702 & 0.089202 & 340.785312 & 337.1333 & 0.089542 \\
\hline$P 4$ & 338.039859 & 334.6454 & 0.089234 & 337.977182 & 334.6135 & 0.088571 \\
\hline$P 5$ & 340.782258 & 337.1684 & 0.088613 & 340.836708 & 337.1315 & 0.090732 \\
\hline$P 6$ & 347.261616 & 344.2088 & 0.064594 & 347.278141 & 344.1655 & 0.065837 \\
\hline$P 7$ & 347.284074 & 344.2132 & 0.064945 & 347.257247 & 344.1705 & 0.065318 \\
\hline$P 8$ & 340.843740 & 337.1693 & 0.089963 & 340.779106 & 337.1328 & 0.089416 \\
\hline$P 9$ & 340.732886 & 337.1713 & 0.087438 & 340.788341 & 337.1343 & 0.089585 \\
\hline$P 10$ & 347.211582 & 344.2014 & 0.063759 & 347.228240 & 344.1582 & 0.065004 \\
\hline$P 11$ & 347.234551 & 344.2034 & 0.064172 & 347.206852 & 344.1601 & 0.064540 \\
\hline$P 12$ & 340.795755 & 337.1818 & 0.088587 & 340.729384 & 337.1449 & 0.088007 \\
\hline$P 13$ & 337.840758 & 334.6379 & 0.084640 & 337.897309 & 334.6060 & 0.086848 \\
\hline$P 14$ & 340.651459 & 337.1766 & 0.085479 & 340.668052 & 337.1395 & 0.086765 \\
\hline$P 15$ & 340.674305 & 337.1724 & 0.086096 & 340.646639 & 337.1354 & 0.086384 \\
\hline$P 16$ & 337.904674 & 334.6419 & 0.086078 & 337.837056 & 334.6099 & 0.085291 \\
\hline
\end{tabular}


Table 6.8: Output Temperatures for Detailed and Two Sub-models and Corresponding Errors of Four-Package Model with Back-side BC and $\alpha=10^{-8}$ $\mathbf{W} / \mu \mathbf{m}^{2} \mathbf{K}$

\begin{tabular}{|c|c|c|c|c|c|c|}
\hline & \multicolumn{3}{|c|}{ Model -1 } & \multicolumn{3}{c|}{ Model -2 } \\
\hline Output & T full & T_sub & Error & T full & T_sub & Error \\
\hline & $\mathbf{( K )}$ & $\mathbf{( K )}$ & & $\mathbf{( K )}$ & $\mathbf{( K )}$ & \\
\hline$T-$ max & 345.717516 & 344.3000 & 0.031006 & 345.717516 & 344.3551 & 0.029801 \\
\hline$P 1$ & 322.352566 & 320.2384 & 0.094583 & 322.285907 & 320.1739 & 0.094769 \\
\hline$P 2$ & 326.169762 & 323.6613 & 0.095853 & 326.091739 & 323.5894 & 0.095905 \\
\hline$P 3$ & 326.177704 & 323.6583 & 0.096242 & 326.086656 & 323.5864 & 0.095844 \\
\hline$P 4$ & 322.373788 & 320.2412 & 0.095316 & 322.271402 & 320.1768 & 0.094049 \\
\hline$P 5$ & 326.244578 & 323.6546 & 0.098686 & 326.177442 & 323.5827 & 0.099121 \\
\hline$P 6$ & 335.144228 & 333.3477 & 0.051119 & 335.064575 & 333.2648 & 0.051327 \\
\hline$P 7$ & 335.152583 & 333.3325 & 0.051777 & 335.059220 & 333.2489 & 0.051636 \\
\hline$P 8$ & 326.266237 & 323.6447 & 0.099806 & 326.162442 & 323.5722 & 0.099006 \\
\hline$P 9$ & 326.251248 & 323.6573 & 0.098812 & 326.184300 & 323.5855 & 0.099250 \\
\hline$P 10$ & 335.151051 & 333.3484 & 0.051283 & 335.071447 & 333.2654 & 0.051496 \\
\hline$P 11$ & 335.159370 & 333.3513 & 0.051425 & 335.065900 & 333.2685 & 0.051258 \\
\hline$P 12$ & 326.272799 & 323.6494 & 0.099852 & 326.168767 & 323.5774 & 0.099025 \\
\hline$P 13$ & 322.371430 & 320.2457 & 0.095020 & 322.305300 & 320.1813 & 0.095224 \\
\hline$P 14$ & 326.188554 & 323.6625 & 0.096456 & 326.110667 & 323.5910 & 0.096500 \\
\hline$P 15$ & 326.196382 & 323.6604 & 0.096807 & 326.105048 & 323.5885 & 0.096401 \\
\hline$P 16$ & 322.392312 & 320.2459 & 0.095855 & 322.289256 & 320.1816 & 0.094559 \\
\hline & & & & & & \\
\hline
\end{tabular}

It has been observed from the above tables that the errors are within satisfactory limit (within $10 \%$ for both right-side and back-side $\mathrm{BC}$ ) when the detailed model is represented with four sub-models with right-side and back-side $\mathrm{BC}$.

Figure 6.9 shows the temperature of the sub-models when the detailed model of the package model is represented with four sub-models. This figure also shows that the maximum temperature (appearing as red) is reached at the centre of each sub-model.

Figure 6.10 shows the temperature of the sub-model for right-side $\mathrm{BC}$. 


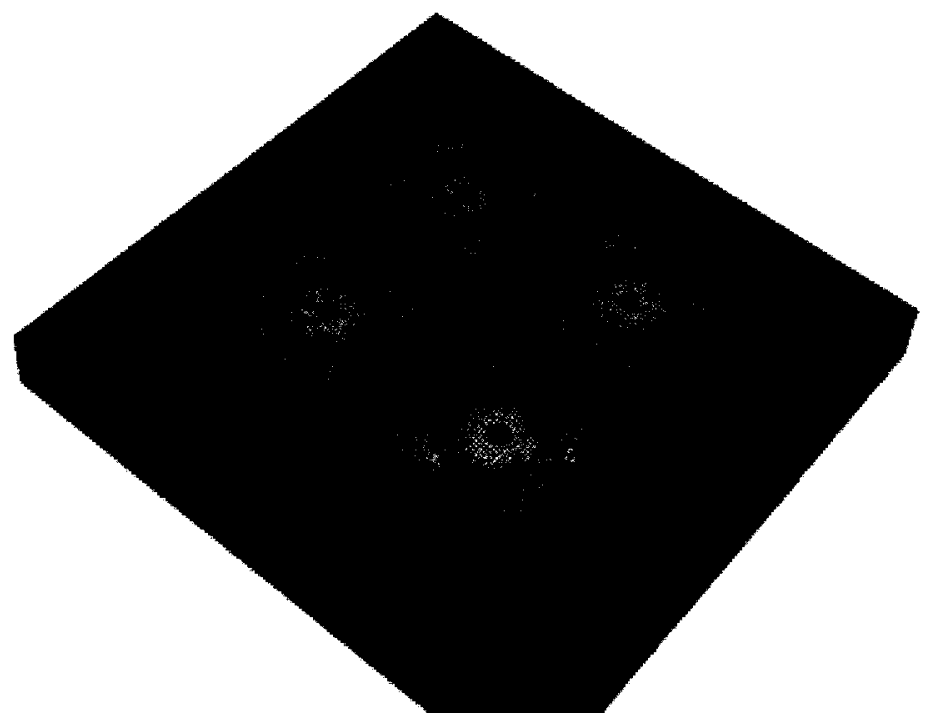

6.9: Temperature of Sub-models for Package Model 


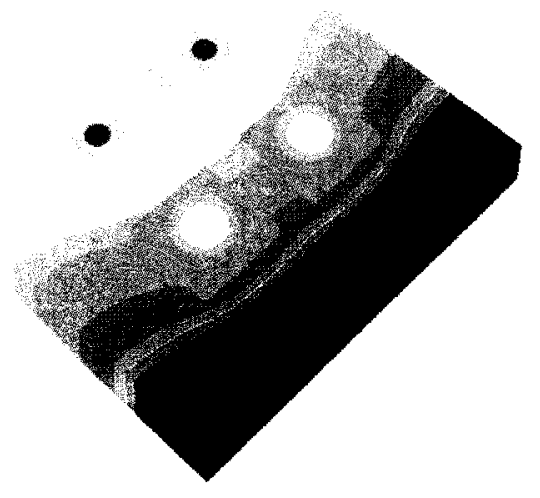

(a)

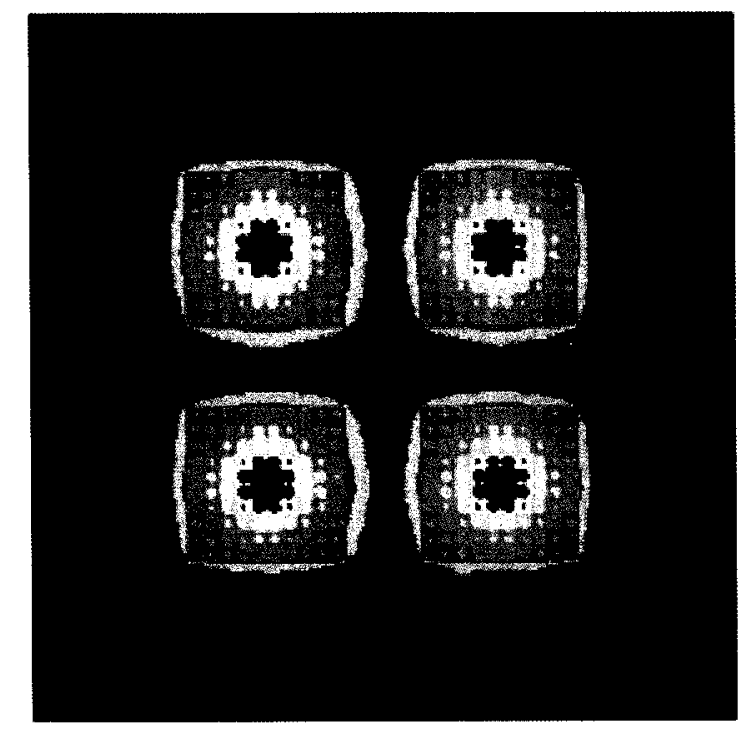

(b)

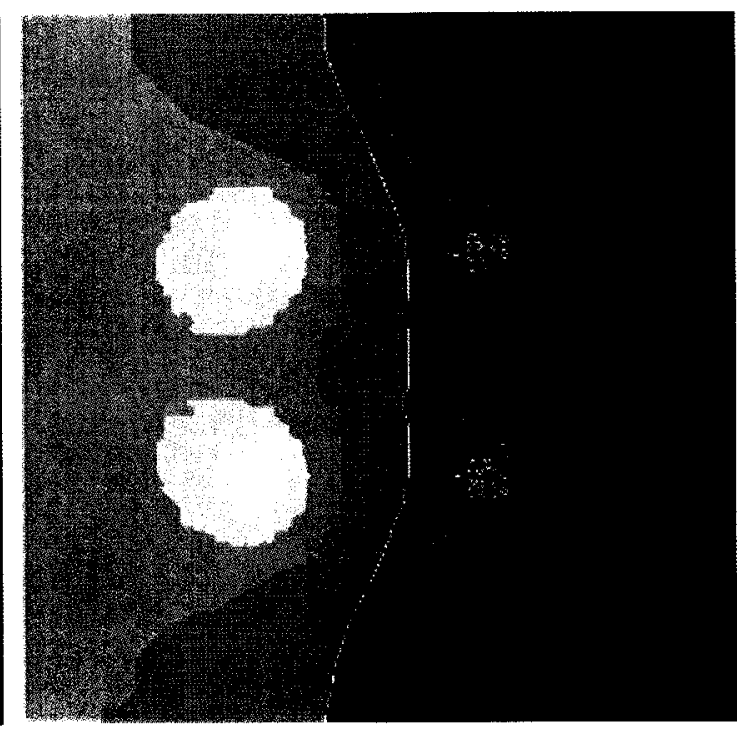

(c)

Figure 6.10: (a) Temperature Contours for Sub-models of Package Model (b) Temperature Contours for Sub-models of Package Model for Right-side BC (c) Temperatures of Package Model for Right-side BC 
Table 6.9 shows the temperature rise and maximum error while using linear and nonlinear substrate. The errors presented here indicate that the primary source of error is not due to the use of a non-linear substrate but just due to the use of sub-models and the portconfiguration selected. The large errors of 59 and $60 \%$ for the case of backside BC are due to the thin substrate placing a strong constraint on the temperature profile of the substrate near the attached sub-models. For this case accurate solutions can only be obtained by using a sub-model with more ports.

Table 6.9: Temperature Rise and Maximum Error for Linear and Non-linear Substrate of Package Model

\begin{tabular}{|c|c|c|c|}
\hline Package & Substrate & Absolute Error (K) & Maximum Error (\%) \\
\hline Single-package & linear & 2.40 & 15.8 \\
\cline { 2 - 4 } Right-side BC & non-linear & 2.40 & 16.0 \\
\hline Four-packages & linear & 6.01 & 18.1 \\
\cline { 2 - 4 } Right-side BC & non-linear & 5.56 & 16.9 \\
\hline Four-packages & linear & 5.30 & 59.0 \\
\cline { 2 - 4 } Back-side BC & non-linear & 5.40 & 60.0 \\
\hline
\end{tabular}

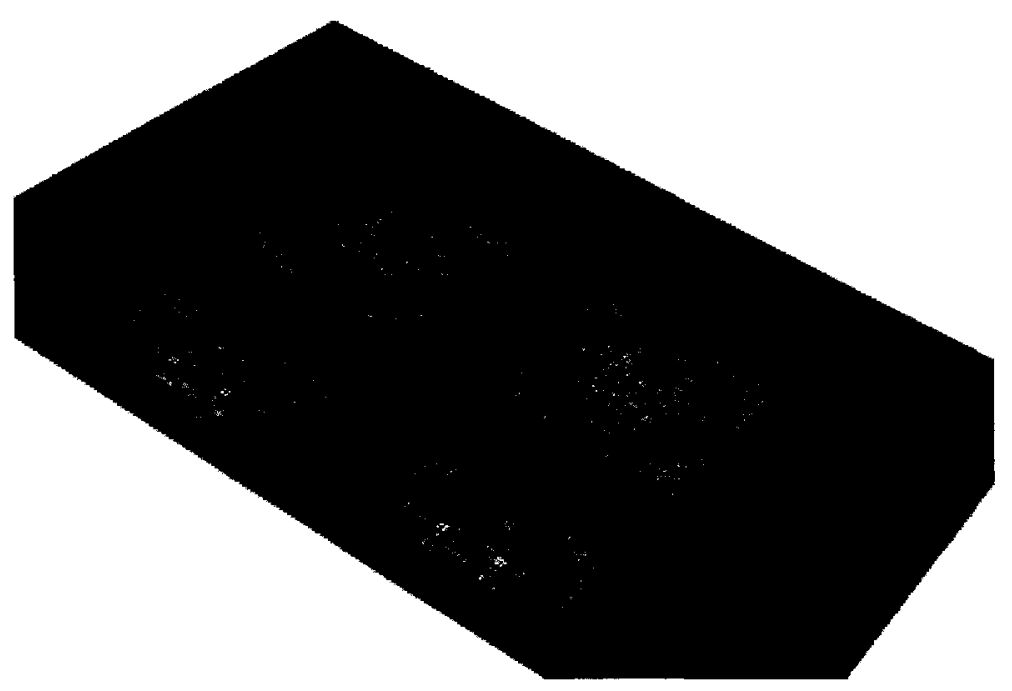

6.11: Temperature Errors of Sub-models for Package Model 
Figure 6.11 shows the errors in temperature of the sub-model when the detailed model is represented with four-package models. The red color at the centre of each sub-model denotes that the maximum error for each sub-model is reached at the centre (inner ring) of the region where the sub-model is attached.

\subsection{Speed-up Using Mixed-model}

In order to clearly show the advantage of using sub-models the simulation time of the three examples presented above have been collected. Table 6.10 shows the simulation time and the number of blocks for full and mixed-model for each of the three package models.

Table 6.10: Simulation Time and Number of Blocks for Full-model and Mixedmodel

\begin{tabular}{|c|c|c|c|c|}
\hline & \multicolumn{2}{|c|}{ Time (sec.) } & \multicolumn{2}{c|}{ Number of Blocks } \\
\hline Model & Full & Mixed & Full & Mixed \\
\hline GaN & 420.0 & 5.110 & 168221 & 18213 \\
\hline GaAs & 1380.9 & 20.850 & 270156 & 69876 \\
\hline Package & 100.5 & 0.669 & 80064 & 11480 \\
\hline
\end{tabular}

It has been observed from Table 6.10 that the model simulation is significantly faster ( 60 - 150 times approximately) when a model is represented with a mixed-model. The speedup is determined by the number of blocks in the base-model and fully detailed model. This shows the need to correctly define the sub-models to be used and the geometry of the base-model. The trade-off that is present in the sub-models is inability to predict a 
detailed temperature distribution in the component and the introduction of error by the use of ports. There also is, of course, the need to create the non-linear sub-model.

\subsection{Summary}

This chapter shows that the detailed model of all the three package models can be effectively represented with a number of sub-models. Mixed-model simulation is significantly faster than that of the original detailed model. In the next chapter the conclusions of this thesis as well as the future research directions are stated. 


\section{Chapter 7}

\section{Conclusions}

This chapter summarizes the work presented in this thesis. Also, it provides a direction of future research work.

\subsection{Summary}

It is very difficult to mathematically represent the non-linear thermal behavior of a detailed package model. In this thesis, non-linear thermal modeling based on neural network approach has been utilized to represent a detailed thermal model. The complex part of a detailed model has been replaced with a simple relationship by representing the detailed model with a mixed-model consisting of a base-model and a number of submodels. The specific tasks performed to represent a detailed non-linear model of the three package models, as mentioned in the thesis, are as follows:

- First, linear thermal simulations have been performed on both detailed componentmodel and sub-models with different port configurations for three package models by using Atar for a variety of $\mathrm{BC}$ applied to the base-model. The accuracy of the sub- 
models has been determined by calculating the errors at different temperatures of interest with respect to that of the detailed model. By using sub-modeling approach, specifically, by comparing the accuracy of sub-models with various $B C$, the appropriate port configuration, i.e., the number of inputs for neural network modeling has been successfully determined.

- Secondly, after deciding the number of inputs, neural network models have been developed for each of the three package models by using a neural network-based modeling tool namely, Neuromodeler. This has been done by training the model with a set of training data that was generated by performing non-linear simulation of Atar. Each of the three package models has been trained using both back propagation and conjugate gradient training method for three-layer as well as four-layer perceptrons network. After comparing the training error, it has been observed that all the models were trained well for three-layer perceptions network for both back propagation and conjugate gradient training method. Four-layer perceptrons network was found to be difficult to train.

- Next, the accuracy of the trained models has been tested with a set of testing data generated by Atar. It has been observed that the average and maximum testing error for all the models were within satisfactory limit. This indicates that the models developed using Neuromodeler is able to represent all the three package models with sufficient accuracy. 
- Finally, a detailed model of each of the three package models has been represented by a number of sub-models. Temperatures at different points of interest of the submodels have been determined in an iterative way by utilizing the MATLAB/C code generated by Neuromodeler. This code essentially represents the neural models of the package models under consideration. These temperatures of various points of the submodels have been compared with that of the detailed model. It has been observed that the calculated errors of the temperatures are within satisfactory limit. This indicates that the overall thermal behavior of a detailed model can be represented with sufficient accuracy by partitioning it into a number of sub-models.

\subsection{Future Research Directions}

There are a number of improvements that could be undertaken in order to achieve even better results using the methodology presented in this thesis. This research can be improved and extended in the following ways:

- There is a scope for further research to investigate other possible ways of port selection for the sub-model.

- The training error of the neural model might be reduced by adjusting various parameters; for example, momentum factor and learning rate of back propagation training algorithm. Moreover, better results may be achieved by adjusting the number of hidden neurons of the neural network.

- Rules such as $\sum I_{p}+P=0$ might be used in neural network. 
- Investigation of transient thermal modeling utilizing neural networks would be a very challenging problem. 


\section{Appendix A}

\section{Simulation Results}

\section{A.1 GaN Based Power Package Amplifier}

Table A.1.1: The Output Temperatures of GaN Model (Case - 1 in Table 4.1)

\begin{tabular}{|c|c|c|c|c|c|c|c|}
\hline Points & T_detail & T_9port & T 7port & T_5port & T 4port & T_2port & T 1port \\
\hline & (K) & (K) & (K) & (K) & (K) & $(\mathbf{K})$ & $(\mathrm{K})$ \\
\hline$I I I$ & 309.32 & 309.68 & 309.64 & 309.55 & 309.64 & 309.57 & 309.57 \\
\hline$I 12$ & 310.35 & 310.61 & 310.61 & 310.53 & 310.59 & 310.53 & 310.53 \\
\hline 113 & 309.53 & 309.84 & 309.81 & 309.77 & 309.78 & 309.74 & 309.74 \\
\hline 114 & 309.86 & 310.14 & 310.05 & 309.96 & 310.06 & 309.98 & 309.97 \\
\hline$I 15$ & 311.12 & 311.27 & 311.24 & 311.17 & 311.23 & 311.17 & 311.17 \\
\hline$I 16$ & 310.10 & 310.32 & 310.23 & 310.19 & 310.20 & 310.16 & 310.16 \\
\hline 121 & 310.30 & 310.30 & 310.17 & 310.07 & 310.19 & 310.09 & 310.08 \\
\hline 122 & 311.63 & 311.49 & 311.43 & 311.36 & 311.42 & 311.35 & 311.35 \\
\hline 123 & 310.54 & 310.47 & 310.34 & 310.30 & 310.31 & 310.27 & 310.27 \\
\hline$I 24$ & 310.51 & 310.43 & 310.29 & 310.18 & 310.31 & 310.20 & 310.20 \\
\hline 125 & 311.45 & 311.29 & 311.21 & 311.14 & 311.21 & 311.14 & 311.14 \\
\hline 126 & 310.55 & 310.46 & 310.33 & 310.30 & 310.31 & 310.28 & 310.27 \\
\hline 131 & 310.03 & 310.28 & 310.16 & 310.07 & 310.19 & 310.09 & 310.09 \\
\hline 132 & 311.24 & 311.35 & 311.28 & 311.22 & 311.29 & 311.22 & 311.22 \\
\hline 133 & 310.23 & 310.43 & 310.33 & 310.32 & 310.31 & 310.29 & 310.29 \\
\hline 134 & 309.80 & 310.02 & 309.93 & 309.84 & 309.96 & 309.87 & 309.87 \\
\hline 135 & 310.51 & 310.63 & 310.57 & 310.51 & 310.58 & 310.51 & 310.51 \\
\hline 136 & 309.78 & 309.99 & 309.90 & 309.91 & 309.90 & 309.89 & 309.88 \\
\hline$P M 1$ & 306.50 & 306.67 & 306.10 & 305.72 & 306.12 & 305.73 & 305.65 \\
\hline$P M 2$ & 306.60 & 306.50 & 305.83 & 305.55 & 305.79 & 305.54 & 305.43 \\
\hline$P M 3$ & 302.82 & 302.71 & 302.69 & 305.28 & 302.69 & 305.28 & 305.13 \\
\hline$P B 1$ & 305.31 & 305.79 & 305.85 & 305.76 & 305.86 & 305.80 & 305.80 \\
\hline$P B 2$ & 305.42 & 305.77 & 305.81 & 305.75 & 305.77 & 305.70 & 305.71 \\
\hline$P B 3$ & 305.44 & 305.83 & 305.79 & 305.73 & 305.84 & 305.77 & 305.78 \\
\hline PBA & 305.56 & 305.83 & 305.79 & 305.75 & 305.77 & 305.71 & 305.72 \\
\hline
\end{tabular}


Table A.1.2: Calculated Errors for Output Temperatures of GaN Model (Case - 1 in Table 4.1)

\begin{tabular}{|c|c|c|c|c|c|c|}
\hline Points & E 9ports & E_7ports & E_5ports & E 4ports & E 2ports & E_1port \\
\hline 111 & 0.038627 & 0.034335 & 0.024678 & 0.034335 & 0.026824 & 0.026824 \\
\hline 112 & 0.025121 & 0.025121 & 0.017391 & 0.023188 & 0.017391 & 0.017391 \\
\hline 113 & 0.032529 & 0.029381 & 0.025184 & 0.026233 & 0.022036 & 0.022036 \\
\hline II 4 & 0.028398 & 0.019270 & 0.010142 & 0.020284 & 0.012170 & 0.011156 \\
\hline 115 & 0.013489 & 0.010791 & 0.004496 & 0.009892 & 0.004496 & 0.004496 \\
\hline 116 & 0.021782 & 0.012871 & 0.008911 & 0.009901 & 0.005941 & 0.005941 \\
\hline 121 & 0.000000 & 0.012621 & 0.022330 & 0.010680 & 0.020388 & 0.021359 \\
\hline 122 & 0.012038 & 0.017197 & 0.023216 & 0.018057 & 0.024076 & 0.024076 \\
\hline 123 & 0.006641 & 0.018975 & 0.022770 & 0.021822 & 0.025617 & 0.025617 \\
\hline 124 & 0.007612 & 0.020932 & 0.031399 & 0.019029 & 0.029496 & 0.029496 \\
\hline 125 & 0.013974 & 0.020961 & 0.027074 & 0.020961 & 0.027074 & 0.027074 \\
\hline 126 & 0.008531 & 0.020853 & 0.023697 & 0.022749 & 0.025592 & 0.026540 \\
\hline 131 & 0.024925 & 0.012961 & 0.003988 & 0.015952 & 0.005982 & 0.005982 \\
\hline 132 & 0.009786 & 0.003559 & 0.001779 & 0.004448 & 0.001779 & 0.001779 \\
\hline 133 & 0.019550 & 0.009775 & 0.008798 & 0.007820 & 0.005865 & 0.005865 \\
\hline 134 & 0.022449 & 0.013265 & 0.004082 & 0.016327 & 0.007143 & 0.007143 \\
\hline 135 & 0.011418 & 0.005709 & 0.000000 & 0.006660 & 0.000000 & 0.000000 \\
\hline 136 & 0.021472 & 0.012270 & 0.013292 & 0.012270 & 0.011247 & 0.010225 \\
\hline$P M 1$ & 0.026154 & 0.061538 & 0.120000 & 0.058462 & 0.118462 & 0.130769 \\
\hline$P M 2$ & 0.015152 & 0.116667 & 0.159091 & 0.122727 & 0.160606 & 0.177273 \\
\hline$P M 3$ & 0.039007 & 0.046099 & 0.872340 & 0.046099 & 0.872340 & 0.819149 \\
\hline$P B 1$ & 0.090395 & 0.101695 & 0.084746 & 0.103578 & 0.092279 & 0.092279 \\
\hline$P B 2$ & 0.064576 & 0.071956 & 0.060886 & 0.064576 & 0.051661 & 0.053506 \\
\hline$P B 3$ & 0.071691 & 0.064338 & 0.053309 & 0.073529 & 0.060662 & 0.062500 \\
\hline$P B 4$ & 0.048561 & 0.041367 & 0.034173 & 0.037770 & 0.026978 & 0.028777 \\
\hline
\end{tabular}


Table A.1.3: The Output Temperatures of GaN Model (Case - 3 in Table 4.1)

\begin{tabular}{|c|c|c|c|c|c|c|c|}
\hline Points & Tdetail & T 9port & T 7port & T 5port & T 4port & T 2port & T 1port \\
\hline & $(\mathbf{K})$ & $(\mathbf{K})$ & $(\mathbf{K})$ & $(\mathbf{K})$ & $(\mathbf{K})$ & $(\mathbf{K})$ & $(\mathbf{K})$ \\
\hline$I I 1$ & 310.19 & 310.57 & 310.52 & 310.44 & 310.55 & 310.48 & 310.48 \\
\hline$I 12$ & 311.22 & 311.49 & 311.49 & 311.42 & 311.50 & 311.44 & 311.45 \\
\hline$I 13$ & 310.40 & 310.73 & 310.69 & 310.65 & 310.69 & 310.65 & 310.65 \\
\hline$I 15$ & 310.76 & 311.04 & 310.94 & 310.86 & 310.97 & 310.89 & 310.89 \\
\hline$I 166$ & 312.00 & 312.17 & 312.13 & 312.07 & 312.14 & 312.08 & 312.08 \\
\hline$I 21$ & 311.22 & 311.21 & 311.12 & 311.09 & 311.11 & 311.08 & 311.08 \\
\hline$I 22$ & 312.53 & 312.39 & 311.07 & 310.98 & 311.09 & 311.01 & 311.00 \\
\hline$I 23$ & 311.44 & 311.38 & 311.24 & 312.27 & 312.33 & 312.27 & 312.27 \\
\hline$I 244$ & 311.44 & 311.35 & 311.20 & 311.11 & 311.22 & 311.12 & 311.11 \\
\hline$I 25$ & 312.37 & 312.21 & 312.13 & 312.07 & 312.12 & 312.06 & 312.06 \\
\hline$I 26$ & 311.48 & 311.38 & 311.24 & 311.22 & 311.22 & 311.20 & 311.19 \\
\hline$I 31$ & 310.98 & 311.22 & 311.09 & 311.01 & 311.10 & 311.01 & 311.00 \\
\hline$I 32$ & 312.18 & 312.29 & 312.21 & 312.16 & 312.20 & 312.14 & 312.14 \\
\hline$I 33$ & 311.18 & 311.36 & 311.25 & 311.25 & 311.23 & 311.21 & 311.21 \\
\hline$I 34$ & 310.76 & 310.97 & 310.86 & 310.79 & 310.87 & 310.79 & 310.78 \\
\hline$I 35$ & 311.47 & 311.58 & 311.50 & 311.45 & 311.49 & 311.43 & 311.43 \\
\hline$I 36$ & 310.74 & 310.93 & 310.84 & 310.85 & 310.81 & 310.81 & 310.80 \\
\hline$P M 1$ & 307.46 & 307.61 & 307.01 & 306.65 & 307.04 & 306.68 & 306.56 \\
\hline$P M 2$ & 307.51 & 307.42 & 306.72 & 306.49 & 306.70 & 306.49 & 306.35 \\
\hline$P M 3$ & 304.00 & 303.89 & 303.87 & 306.23 & 303.86 & 306.24 & 306.04 \\
\hline$P B 1$ & 306.17 & 306.67 & 306.73 & 306.66 & 306.77 & 306.71 & 306.72 \\
\hline$P B 2$ & 306.27 & 306.64 & 306.68 & 306.63 & 306.67 & 306.62 & 306.63 \\
\hline$P B 3$ & 306.42 & 306.79 & 306.74 & 306.69 & 306.75 & 306.69 & 306.70 \\
\hline$P B 4$ & 306.52 & 306.78 & 306.73 & 306.70 & 306.68 & 306.63 & 306.63 \\
\hline
\end{tabular}

Table A.1.4: Calculated Errors for Output Temperatures of GaN Model (Case - 3 in Table 4.1)

\begin{tabular}{|l|l|l|l|l|l|l|}
\hline Points & E_9ports & E_7ports & E_5ports & E_4ports & E_2ports & E_ 1port \\
\hline & & & & & & \\
\hline$I I I$ & 0.037291 & 0.032385 & 0.024534 & 0.035329 & 0.028459 & 0.028459 \\
\hline$I 12$ & 0.024064 & 0.024064 & 0.017825 & 0.024955 & 0.019608 & 0.020499 \\
\hline$I 13$ & 0.031731 & 0.027885 & 0.024038 & 0.027885 & 0.024038 & 0.024038 \\
\hline$I I 4$ & 0.026022 & 0.016729 & 0.009294 & 0.019517 & 0.012082 & 0.012082 \\
\hline$I 15$ & 0.014167 & 0.010833 & 0.005833 & 0.011667 & 0.006667 & 0.006667 \\
\hline$I 16$ & 0.020018 & 0.011829 & 0.009099 & 0.010919 & 0.008189 & 0.008189 \\
\hline$I 21$ & 0.000000 & 0.013369 & 0.021390 & 0.011586 & 0.018717 & 0.019608 \\
\hline$I 22$ & 0.011173 & 0.015962 & 0.020750 & 0.015962 & 0.020750 & 0.020750 \\
\hline$I 23$ & 0.005245 & 0.017483 & 0.020105 & 0.019231 & 0.021853 & 0.021853 \\
\hline$I 24$ & 0.007867 & 0.020979 & 0.028846 & 0.019231 & 0.027972 & 0.028846 \\
\hline$I 25$ & 0.012935 & 0.019402 & 0.024252 & 0.020210 & 0.025061 & 0.025061 \\
\hline
\end{tabular}




\begin{tabular}{|l|l|l|l|l|l|l|}
\hline$I 26$ & 0.008711 & 0.020906 & 0.022648 & 0.022648 & 0.024390 & 0.025261 \\
\hline$I 3 \_1$ & 0.021858 & 0.010018 & 0.002732 & 0.010929 & 0.002732 & 0.001821 \\
\hline$I 3 \_2$ & 0.009031 & 0.002463 & 0.001642 & 0.001642 & 0.003284 & 0.003284 \\
\hline$I 33$ & 0.016100 & 0.006261 & 0.006261 & 0.004472 & 0.002683 & 0.002683 \\
\hline$I 344$ & 0.019517 & 0.009294 & 0.002788 & 0.010223 & 0.002788 & 0.001859 \\
\hline$I 355$ & 0.009590 & 0.002616 & 0.001744 & 0.001744 & 0.003487 & 0.003487 \\
\hline$I 36$ & 0.017691 & 0.009311 & 0.010242 & 0.006518 & 0.006518 & 0.005587 \\
\hline$P M 1$ & 0.020107 & 0.060322 & 0.108579 & 0.056300 & 0.104558 & 0.120643 \\
\hline$P M 2$ & 0.011984 & 0.105193 & 0.135819 & 0.107856 & 0.135819 & 0.154461 \\
\hline$P M 3$ & 0.027500 & 0.032500 & 0.557500 & 0.035000 & 0.560000 & 0.510000 \\
\hline$P B 1$ & 0.081037 & 0.090762 & 0.079417 & 0.097245 & 0.087520 & 0.089141 \\
\hline$P B 2$ & 0.059011 & 0.065391 & 0.057416 & 0.063796 & 0.055821 & 0.057416 \\
\hline$P B 3$ & 0.057632 & 0.049844 & 0.042056 & 0.051402 & 0.042056 & 0.043614 \\
\hline$P B 4$ & 0.039877 & 0.032209 & 0.027607 & 0.024540 & 0.016871 & 0.016871 \\
\hline
\end{tabular}

Table A.1.5: The Output Temperatures of GaN Model (Case - 4 in Table 4.1)

\begin{tabular}{|c|c|c|c|c|c|c|c|}
\hline Points & T_detail & T 9port & T_7port & T 5port & T 4port & T_2port & $T$ 1port \\
\hline & (K) & (K) & (K) & (K) & (K) & $(\mathbf{K})$ & (K) \\
\hline II 1 & 310.47 & 310.81 & 310.77 & 310.65 & 310.73 & 310.64 & 310.64 \\
\hline$I 12$ & 311.49 & 311.73 & 311.73 & 311.64 & 311.68 & 311.60 & 311.60 \\
\hline$I 13$ & 310.66 & 310.96 & 310.93 & 310.87 & 310.87 & 310.81 & 310.81 \\
\hline$I 14$ & 311.00 & 311.26 & 311.18 & 311.06 & 311.15 & 311.05 & 311.05 \\
\hline I1 5 & 312.25 & 312.39 & 312.36 & 312.27 & 312.33 & 312.24 & 312.24 \\
\hline 116 & 311.22 & 311.42 & 311.34 & 311.28 & 311.29 & 311.24 & 311.23 \\
\hline 121 & 311.41 & 311.41 & 311.28 & 311.15 & 311.28 & 311.16 & 311.16 \\
\hline 122 & 312.73 & 312.59 & 312.53 & 312.44 & 312.52 & 312.43 & 312.43 \\
\hline 123 & 311.63 & 311.56 & 311.44 & 311.38 & 311.40 & 311.35 & 311.34 \\
\hline$I 24$ & 311.6 & 311.52 & 311.38 & 311.25 & 311.40 & 311.27 & 311.27 \\
\hline 125 & 312.52 & 312.37 & 312.30 & 312.21 & 312.31 & 312.22 & 312.22 \\
\hline$I 2 \_6$ & 311.62 & 311.53 & 311.41 & 311.37 & 311.40 & 311.35 & 311.35 \\
\hline 131 & 311.10 & 311.36 & 311.24 & 311.12 & 311.28 & 311.16 & 311.16 \\
\hline 132 & 312.29 & 312.42 & 312.35 & 312.27 & 312.38 & 312.29 & 312.29 \\
\hline 133 & 311.28 & 311.49 & 311.39 & 311.37 & 311.40 & 311.37 & 311.36 \\
\hline 134 & 310.85 & 311.09 & 311.00 & 310.89 & 311.05 & 310.94 & 310.94 \\
\hline 135 & 311.55 & 311.69 & 311.63 & 311.55 & 311.67 & 311.59 & 311.59 \\
\hline 136 & 310.80 & 311.03 & 310.96 & 310.95 & 310.98 & 310.96 & 310.96 \\
\hline$P M 1$ & 307.64 & 307.79 & 307.23 & 306.79 & 307.22 & 306.79 & 306.72 \\
\hline$P M 2$ & 307.69 & 307.60 & 306.95 & 306.61 & 306.89 & 306.59 & 306.50 \\
\hline$P M 3$ & 303.52 & 303.42 & 303.40 & 306.33 & 303.40 & 306.31 & 306.20 \\
\hline$P B 1$ & 306.48 & 306.93 & 306.99 & 306.88 & 306.95 & 306.87 & 306.87 \\
\hline$P B 2$ & 306.57 & 306.89 & 306.94 & 306.86 & 306.86 & 306.78 & 306.78 \\
\hline$P B 3$ & 306.47 & 306.89 & 306.85 & 306.77 & 306.93 & 306.85 & 306.85 \\
\hline$P B 4$ & 306.57 & 306.87 & 306.83 & 306.78 & 306.86 & 306.79 & 306.79 \\
\hline
\end{tabular}


Table A.1.6: Calculated Errors for Output Temperatures of GaN Model (Case - 4 in Table 4.1)

\begin{tabular}{|c|c|c|c|c|c|c|}
\hline Points & E 9ports & E 7ports & E 5ports & E 4ports & E 2ports & E 1port \\
\hline II 1 & 0.032474 & 0.028653 & 0.017192 & 0.024833 & 0.016237 & 0.016237 \\
\hline 112 & 0.020888 & 0.020888 & 0.013055 & 0.016536 & 0.009574 & 0.009574 \\
\hline 113 & 0.028143 & 0.025328 & 0.019700 & 0.019700 & 0.014071 & 0.014071 \\
\hline 114 & 0.023636 & 0.016364 & 0.005455 & 0.013636 & 0.004545 & 0.004545 \\
\hline II 5 & 0.011429 & 0.008980 & 0.001633 & 0.006531 & 0.000816 & 0.000816 \\
\hline 116 & 0.017825 & 0.010695 & 0.005348 & 0.006239 & 0.001783 & 0.000891 \\
\hline 121 & 0.000000 & 0.011394 & 0.022787 & 0.011394 & 0.021911 & 0.021911 \\
\hline 122 & 0.010998 & 0.015711 & 0.022781 & 0.016496 & 0.023566 & 0.023566 \\
\hline 123 & 0.006019 & 0.016337 & 0.021496 & 0.019776 & 0.024076 & 0.024936 \\
\hline 124 & 0.006897 & 0.018966 & 0.030172 & 0.017241 & 0.028448 & 0.028448 \\
\hline 125 & 0.011981 & 0.017572 & 0.024760 & 0.016773 & 0.023962 & 0.023962 \\
\hline$I 26$ & 0.007745 & 0.018072 & 0.021515 & 0.018933 & 0.023236 & 0.023236 \\
\hline 131 & 0.023423 & 0.012613 & 0.001802 & 0.016216 & 0.005405 & 0.005405 \\
\hline 132 & 0.010578 & 0.004882 & 0.001627 & 0.007323 & 0.000000 & 0.000000 \\
\hline 133 & 0.018617 & 0.009752 & 0.007979 & 0.010638 & 0.007979 & 0.007092 \\
\hline 134 & 0.022120 & 0.013825 & 0.003687 & 0.018433 & 0.008295 & 0.008295 \\
\hline 135 & 0.012121 & 0.006926 & 0.000000 & 0.010390 & 0.003463 & 0.003463 \\
\hline 136 & 0.021296 & 0.014815 & 0.013889 & 0.016667 & 0.014815 & 0.014815 \\
\hline$P M 1$ & 0.019634 & 0.053665 & 0.111257 & 0.054974 & 0.111257 & 0.120419 \\
\hline$P M 2$ & 0.011704 & 0.096229 & 0.140442 & 0.104031 & 0.143043 & 0.154746 \\
\hline$P M 3$ & 0.028409 & 0.034091 & 0.798295 & 0.034091 & 0.792614 & 0.761364 \\
\hline$P B 1$ & 0.069444 & 0.078704 & 0.061728 & 0.072531 & 0.060185 & 0.060185 \\
\hline$P B 2$ & 0.048706 & 0.056317 & 0.044140 & 0.044140 & 0.031963 & 0.031963 \\
\hline$P B 3$ & 0.064915 & 0.058733 & 0.046368 & 0.071097 & 0.058733 & 0.058733 \\
\hline$P B 4$ & 0.045662 & 0.039574 & 0.031963 & 0.044140 & 0.033486 & 0.033486 \\
\hline
\end{tabular}

Table A.1.7: The Output Temperatures of GaN Model (Case - 5 in Table 4.1)

\begin{tabular}{|c|c|c|c|c|c|c|c|}
\hline Points & T detail & T 9port & T 7port & T 5port & T_4port & T_2port & T 1port \\
\hline & $(\mathbf{K})$ & $(\mathbf{K})$ & $(\mathbf{K})$ & $(\mathbf{K})$ & $(\mathbf{K})$ & (K) & (K) \\
\hline$I 1 \quad 1$ & 310.46 & 310.81 & 310.77 & 310.66 & 310.74 & 310.66 & 310.66 \\
\hline 112 & 311.45 & 311.71 & 311.71 & 311.63 & 311.69 & 311.62 & 311.62 \\
\hline 113 & 310.59 & 310.91 & 310.88 & 310.83 & 310.87 & 310.83 & 310.83 \\
\hline II 4 & 311.01 & 311.27 & 311.18 & 311.07 & 311.16 & 311.07 & 311.07 \\
\hline 115 & 312.22 & 312.37 & 312.34 & 312.26 & 312.33 & 312.26 & 312.26 \\
\hline 116 & 311.15 & 311.38 & 311.30 & 311.26 & 311.29 & 311.25 & 311.25 \\
\hline 121 & 311.44 & 311.43 & 311.30 & 311.18 & 311.28 & 311.18 & 311.17 \\
\hline$I 22$ & 312.72 & 312.58 & 312.52 & 312.45 & 312.52 & 312.45 & 312.44 \\
\hline 123 & 311.59 & 311.53 & 311.40 & 311.37 & 311.41 & 311.36 & 311.36 \\
\hline 124 & 311.65 & 311.55 & 311.41 & 311.29 & 311.41 & 311.29 & 311.29 \\
\hline
\end{tabular}




\begin{tabular}{|l|l|l|l|l|l|l|l|}
\hline$I 25$ & 312.54 & 312.38 & 312.31 & 312.23 & 312.31 & 312.23 & 312.23 \\
\hline$I 26$ & 311.60 & 311.52 & 311.39 & 311.37 & 311.40 & 311.37 & 311.36 \\
\hline$I 3 \quad 1$ & 311.16 & 311.40 & 311.28 & 311.18 & 311.29 & 311.18 & 311.18 \\
\hline$I 32$ & 312.33 & 312.45 & 312.37 & 312.31 & 312.38 & 312.31 & 312.31 \\
\hline$I 33$ & 311.28 & 311.49 & 311.39 & 311.38 & 311.41 & 311.38 & 311.38 \\
\hline$I 34$ & 310.93 & 311.14 & 311.05 & 310.95 & 311.06 & 310.96 & 310.96 \\
\hline$I 35$ & 311.60 & 311.73 & 311.66 & 311.60 & 311.67 & 311.61 & 311.61 \\
\hline$I 36$ & 310.82 & 311.04 & 310.97 & 310.97 & 310.99 & 310.98 & 310.98 \\
\hline$P M 1$ & 307.72 & 307.85 & 307.26 & 306.83 & 307.24 & 306.82 & 306.74 \\
\hline$P M 2$ & 307.56 & 307.49 & 306.85 & 306.63 & 306.85 & 306.63 & 306.52 \\
\hline$P M 3$ & 303.85 & 303.75 & 303.72 & 306.37 & 303.73 & 306.36 & 306.22 \\
\hline$P B 1$ & 306.48 & 306.93 & 306.99 & 306.90 & 306.96 & 306.89 & 306.89 \\
\hline$P B 2$ & 306.46 & 306.82 & 306.86 & 306.80 & 306.86 & 306.79 & 306.80 \\
\hline$P B 3$ & 306.59 & 306.97 & 306.92 & 306.85 & 306.94 & 306.87 & 306.87 \\
\hline$P B 4$ & 306.57 & 306.87 & 306.83 & 306.79 & 306.87 & 306.80 & 306.81 \\
\hline
\end{tabular}

Table A.1.8: Calculated Errors for Output Temperatures of GaN Model (Case - 5 in Table 4.1)

\begin{tabular}{|c|c|c|c|c|c|c|}
\hline Points & E_9ports & E_7ports & E_5ports & E_4ports & E_2ports & E 1port \\
\hline & & & & & & \\
\hline$I 11$ & 0.033461 & 0.029637 & 0.019120 & 0.026769 & 0.019120 & 0.019120 \\
\hline$I I 2$ & 0.022707 & 0.022707 & 0.015721 & 0.020961 & 0.014847 & 0.014847 \\
\hline$I 13$ & 0.030217 & 0.027384 & 0.022663 & 0.026440 & 0.022663 & 0.022663 \\
\hline$I 14$ & 0.023615 & 0.015441 & 0.005450 & 0.013624 & 0.005450 & 0.005450 \\
\hline$I 15$ & 0.012275 & 0.009820 & 0.003273 & 0.009002 & 0.003273 & 0.003273 \\
\hline$I 16$ & 0.020628 & 0.013453 & 0.009865 & 0.012556 & 0.008969 & 0.008969 \\
\hline$I 21$ & 0.000874 & 0.012238 & 0.022727 & 0.013986 & 0.022727 & 0.023601 \\
\hline$I 22$ & 0.011006 & 0.015723 & 0.021226 & 0.015723 & 0.021226 & 0.022013 \\
\hline$I 23$ & 0.005177 & 0.016393 & 0.018982 & 0.015531 & 0.019845 & 0.019845 \\
\hline$I 244$ & 0.008584 & 0.020601 & 0.030901 & 0.020601 & 0.030901 & 0.030901 \\
\hline$I 25$ & 0.012759 & 0.018341 & 0.024721 & 0.018341 & 0.024721 & 0.024721 \\
\hline$I 26$ & 0.006897 & 0.018103 & 0.019828 & 0.017241 & 0.019828 & 0.020690 \\
\hline$I 31$ & 0.021505 & 0.010753 & 0.001792 & 0.011649 & 0.001792 & 0.001792 \\
\hline$I 32$ & 0.009732 & 0.003244 & 0.001622 & 0.004055 & 0.001622 & 0.001622 \\
\hline$I 3$ 3 & 0.018617 & 0.009752 & 0.008865 & 0.011525 & 0.008865 & 0.008865 \\
\hline$I 34$ & 0.019213 & 0.010979 & 0.001830 & 0.011894 & 0.002745 & 0.002745 \\
\hline$I 35$ & 0.011207 & 0.005172 & 0.000000 & 0.006034 & 0.000862 & 0.000862 \\
\hline$I 36$ & 0.020333 & 0.013863 & 0.013863 & 0.015712 & 0.014787 & 0.014787 \\
\hline$P M 1$ & 0.016839 & 0.059585 & 0.115285 & 0.062176 & 0.116580 & 0.126943 \\
\hline$P M 2$ & 0.009259 & 0.093915 & 0.123016 & 0.093915 & 0.123016 & 0.137566 \\
\hline$P M 3$ & 0.025974 & 0.033766 & 0.654545 & 0.031169 & 0.651948 & 0.615584 \\
\hline$P B 1$ & 0.069444 & 0.078704 & 0.064815 & 0.074074 & 0.063272 & 0.063272 \\
\hline$P B 2$ & 0.055728 & 0.061920 & 0.052632 & 0.061920 & 0.051084 & 0.052632 \\
\hline$P B 3$ & 0.057663 & 0.050076 & 0.039454 & 0.053111 & 0.042489 & 0.042489 \\
\hline$P B 4$ & 0.045662 & 0.039574 & 0.033486 & 0.045662 & 0.035008 & 0.036530 \\
\hline
\end{tabular}


Table A.1.9: The Output Temperatures of GaN Model (Case - 6 in Table 4.1)

\begin{tabular}{|c|c|c|c|c|c|c|c|}
\hline Points & T_detail & T_9port & T_7port & T_5port & T_4port & T_2port & T_1port \\
\hline & $(\mathbf{K})$ & $(\mathbf{K})$ & $(\mathbf{K})$ & $(\mathbf{K})$ & $(\mathbf{K})$ & $(\mathbf{K})$ & $(\mathbf{K})$ \\
\hline$I I 1$ & 310.16 & 310.53 & 310.49 & 310.41 & 310.51 & 310.44 & 310.44 \\
\hline$I 13$ & 311.23 & 311.48 & 311.49 & 311.42 & 311.46 & 311.40 & 311.41 \\
\hline$I 14$ & 310.46 & 310.75 & 310.72 & 310.67 & 310.65 & 310.61 & 310.61 \\
\hline$I 15$ & 312.00 & 310.99 & 310.91 & 310.82 & 310.93 & 310.85 & 310.85 \\
\hline$I 16$ & 311.03 & 311.22 & 311.14 & 311.10 & 311.08 & 311.04 & 311.04 \\
\hline$I 21$ & 311.14 & 311.15 & 311.02 & 310.93 & 311.06 & 310.97 & 310.96 \\
\hline$I 22$ & 312.50 & 312.36 & 312.30 & 312.24 & 312.30 & 312.23 & 312.23 \\
\hline$I 23$ & 311.46 & 311.38 & 311.24 & 311.20 & 311.19 & 311.15 & 311.15 \\
\hline$I 244$ & 311.34 & 311.27 & 311.14 & 311.04 & 311.18 & 311.08 & 311.07 \\
\hline$I 25$ & 312.32 & 312.16 & 312.08 & 312.02 & 312.09 & 312.02 & 312.02 \\
\hline$I 26$ & 311.47 & 311.36 & 311.23 & 311.20 & 311.19 & 311.16 & 311.15 \\
\hline$I 31$ & 310.86 & 311.12 & 311.01 & 310.93 & 311.06 & 310.97 & 310.96 \\
\hline$I 32$ & 312.11 & 312.23 & 312.15 & 312.10 & 312.16 & 312.10 & 312.10 \\
\hline$I 33$ & 311.15 & 311.33 & 311.23 & 311.22 & 311.19 & 311.17 & 311.17 \\
\hline$I 34$ & 310.63 & 310.87 & 310.77 & 310.70 & 310.83 & 310.75 & 310.74 \\
\hline$I 35$ & 311.38 & 311.51 & 311.44 & 311.39 & 311.45 & 311.39 & 311.39 \\
\hline$I 36$ & 310.69 & 310.89 & 310.80 & 310.81 & 310.77 & 310.77 & 310.76 \\
\hline$P M 1$ & 307.26 & 307.47 & 306.92 & 306.60 & 306.97 & 306.62 & 306.52 \\
\hline$P M 2$ & 307.59 & 307.47 & 306.78 & 306.45 & 306.69 & 306.43 & 306.31 \\
\hline$P M 3$ & 303.81 & 303.70 & 303.68 & 306.17 & 303.67 & 306.17 & 306.00 \\
\hline$P B 1$ & 306.13 & 306.63 & 306.69 & 306.62 & 306.73 & 306.67 & 306.68 \\
\hline$P B 2$ & 306.37 & 306.69 & 306.74 & 306.67 & 306.64 & 306.58 & 306.59 \\
\hline$P B 3$ & 306.24 & 306.67 & 306.62 & 306.57 & 306.71 & 306.65 & 306.66 \\
\hline$P B 4$ & 306.49 & 306.74 & 306.70 & 306.66 & 306.64 & 306.59 & 306.59 \\
\hline
\end{tabular}

Table A.1.10: Calculated Errors for Output Temperatures of GaN Model (Case - 6 in Table 4.1)

\begin{tabular}{|c|c|c|c|c|c|c|}
\hline Points & E_9ports & E_7ports & E_5ports & E_4ports & E_2ports & E_1port \\
\hline & & & & & & \\
\hline$I 1 / 1$ & 0.036417 & 0.03248 & 0.024606 & 0.034449 & 0.027559 & 0.027559 \\
\hline$I 12$ & 0.022262 & 0.023152 & 0.016919 & 0.020481 & 0.015138 & 0.016028 \\
\hline$I 13$ & 0.027725 & 0.024857 & 0.020076 & 0.018164 & 0.014340 & 0.014340 \\
\hline$I I 4$ & 0.027103 & 0.019626 & 0.011215 & 0.021495 & 0.014019 & 0.014019 \\
\hline$I 15$ & 0.012500 & 0.010000 & 0.004167 & 0.009167 & 0.003333 & 0.004167 \\
\hline$I 166$ & 0.017226 & 0.009973 & 0.006346 & 0.004533 & 0.000907 & 0.000907 \\
\hline$I 21$ & 0.000898 & 0.010772 & 0.018851 & 0.007181 & 0.015260 & 0.016158 \\
\hline$I 2 \_$ & 0.011200 & 0.016000 & 0.020800 & 0.016000 & 0.021600 & 0.021600 \\
\hline$I 233$ & 0.006981 & 0.019197 & 0.022688 & 0.023560 & 0.027051 & 0.027051 \\
\hline$I 244$ & 0.006173 & 0.017637 & 0.026455 & 0.014109 & 0.022928 & 0.023810 \\
\hline
\end{tabular}




\begin{tabular}{|l|l|l|l|l|l|l|}
\hline$I 25$ & 0.012987 & 0.019481 & 0.024351 & 0.018669 & 0.024351 & 0.024351 \\
\hline$I 26$ & 0.009590 & 0.020924 & 0.023540 & 0.024412 & 0.027027 & 0.027899 \\
\hline$I 31$ & 0.023941 & 0.013812 & 0.006446 & 0.018416 & 0.010129 & 0.009208 \\
\hline$I 32$ & 0.009909 & 0.003303 & 0.000826 & 0.004129 & 0.000826 & 0.000826 \\
\hline$I 33$ & 0.016143 & 0.007175 & 0.006278 & 0.003587 & 0.001794 & 0.001794 \\
\hline$I 34$ & 0.022578 & 0.013170 & 0.006585 & 0.018815 & 0.011289 & 0.010348 \\
\hline$I 35$ & 0.011424 & 0.005272 & 0.000879 & 0.006151 & 0.000879 & 0.000879 \\
\hline$I 366$ & 0.018709 & 0.010290 & 0.011225 & 0.007484 & 0.007484 & 0.006548 \\
\hline$P M 1$ & 0.028926 & 0.046832 & 0.090909 & 0.039945 & 0.088154 & 0.101928 \\
\hline$P M 2$ & 0.015810 & 0.106719 & 0.150198 & 0.118577 & 0.152833 & 0.168643 \\
\hline$P M 3$ & 0.028871 & 0.034121 & 0.619423 & 0.036745 & 0.619423 & 0.574803 \\
\hline$P B 1$ & 0.081566 & 0.091354 & 0.079935 & 0.097879 & 0.088091 & 0.089723 \\
\hline$P B 2$ & 0.050235 & 0.058085 & 0.047096 & 0.042386 & 0.032967 & 0.034537 \\
\hline$P B 3$ & 0.068910 & 0.060897 & 0.052885 & 0.075321 & 0.065705 & 0.067308 \\
\hline$P B 4$ & 0.038521 & 0.032357 & 0.026194 & 0.023112 & 0.015408 & 0.015408 \\
\hline
\end{tabular}




\section{A.2 GaAs Based Power Amplifier}

Table A.2.1: The Output Temperatures of GaAs Model (Case - 1 in Table 4.5)

\begin{tabular}{|c|c|c|c|c|c|}
\hline Points & T_detailed & T_9ports & T_5ports & T_2ports & T 1port \\
\hline & $(\mathbf{K})$ & $(\mathbf{K})$ & $(\mathbf{K})$ & $(\mathbf{K})$ & $(\mathbf{K})$ \\
\hline$I I I$ & 631.04 & 627.96 & 627.90 & 627.83 & 626.39 \\
\hline$I I 2$ & 970.91 & 967.70 & 967.61 & 967.42 & 965.86 \\
\hline$I I 3$ & 909.77 & 906.53 & 906.40 & 906.21 & 904.98 \\
\hline$I 21$ & 642.91 & 639.87 & 639.82 & 639.78 & 638.65 \\
\hline$I 22$ & 984.03 & 980.90 & 980.82 & 980.65 & 979.54 \\
\hline$I 23$ & 922.60 & 919.40 & 919.28 & 919.09 & 918.22 \\
\hline$I 31$ & 646.81 & 643.67 & 643.58 & 643.46 & 642.30 \\
\hline$I 32$ & 989.03 & 985.79 & 985.68 & 985.39 & 984.22 \\
\hline$I 33$ & 925.96 & 922.65 & 922.49 & 922.20 & 921.26 \\
\hline$I 41$ & 626.95 & 623.54 & 623.36 & 622.89 & 621.23 \\
\hline$I 42$ & 965.31 & 961.77 & 961.56 & 960.92 & 959.10 \\
\hline$I 43$ & 906.66 & 903.12 & 902.87 & 902.33 & 900.93 \\
\hline$P M 1$ & 338.29 & 333.81 & 333.72 & 332.91 & 316.54 \\
\hline$P M 2$ & 345.59 & 340.37 & 340.18 & 333.51 & 317.18 \\
\hline$P M 3$ & 349.01 & 341.77 & 341.68 & 334.22 & 317.70 \\
\hline$P M 4$ & 323.66 & 320.38 & 320.38 & 331.48 & 315.55 \\
\hline$P B 1$ & 316.89 & 316.37 & 316.46 & 316.49 & 316.92 \\
\hline$P B 2$ & 317.66 & 316.80 & 316.69 & 316.70 & 317.13 \\
\hline$P B 3$ & 317.01 & 316.42 & 316.43 & 316.45 & 316.90 \\
\hline$P B 4$ & 315.71 & 315.74 & 316.06 & 316.11 & 316.52 \\
\hline$P B 5$ & 323.82 & 321.20 & 321.51 & 321.53 & 321.85 \\
\hline
\end{tabular}

Table A.2.2: Calculated Errors for Output Temperatures of GaAs Model (Case - 1 in Table 4.5)

\begin{tabular}{|c|c|c|c|c|}
\hline Points & E_9ports & E_5ports & E_2ports & E_1port \\
\hline & & & & \\
\hline$I I I$ & 0.009304 & 0.009485 & 0.009697 & 0.014047 \\
\hline$I I 2$ & 0.005313 & 0.005527 & 0.005838 & 0.007855 \\
\hline$I 13$ & 0.008865 & 0.009011 & 0.009128 & 0.012423 \\
\hline$I 2 I$ & 0.004576 & 0.004693 & 0.004941 & 0.006564 \\
\hline$I 2-2$ & 0.005140 & 0.005332 & 0.005638 & 0.007035 \\
\hline$I 23$ & 0.009054 & 0.009313 & 0.009659 & 0.013004 \\
\hline$I 31$ & 0.004702 & 0.004862 & 0.005283 & 0.006981 \\
\hline$I 32$ & 0.005288 & 0.005543 & 0.006007 & 0.007508 \\
\hline$I 3 \_3$ & 0.010430 & 0.010980 & 0.012418 & 0.017495 \\
\hline
\end{tabular}




\begin{tabular}{|c|c|c|c|c|}
\hline$I 41$ & 0.005321 & 0.005636 & 0.006598 & 0.009334 \\
\hline$I 42$ & 0.005835 & 0.006247 & 0.007137 & 0.009445 \\
\hline$I 43$ & 0.117002 & 0.119352 & 0.140507 & 0.568033 \\
\hline$P M 1$ & 0.114499 & 0.118666 & 0.264970 & 0.623163 \\
\hline$P M 2$ & 0.147725 & 0.149561 & 0.301775 & 0.638849 \\
\hline$P M 3$ & 0.138631 & 0.138631 & 0.330516 & 0.342773 \\
\hline$P M 4$ & 0.030787 & 0.025459 & 0.023683 & 0.001776 \\
\hline$P B 1$ & 0.048698 & 0.054926 & 0.054360 & 0.030011 \\
\hline$P B 2$ & 0.034685 & 0.034098 & 0.032922 & 0.006467 \\
\hline$P B 3$ & 0.001910 & 0.022279 & 0.025461 & 0.051560 \\
\hline$P B 4$ & 0.109992 & 0.096977 & 0.096138 & 0.082704 \\
\hline$P B 5$ & 0.009304 & 0.009485 & 0.009697 & 0.014047 \\
\hline
\end{tabular}

Table A.2.3: The Output Temperatures of GaAs Model (Case - 2 in Table 4.5)

\begin{tabular}{|c|c|c|c|c|c|}
\hline Points & T_detailed & T 9ports & T_5ports & T_2ports & T_1port \\
\hline & $(\mathbf{K})$ & $(\mathbf{K})$ & $(\mathbf{K})$ & $(\mathbf{K})$ & $(\mathbf{K})$ \\
\hline$I 1 / 2$ & 631.44 & 628.35 & 628.27 & 628.20 & 626.69 \\
\hline$I 13$ & 971.31 & 968.10 & 967.99 & 967.79 & 966.16 \\
\hline$I 21$ & 910.17 & 906.92 & 906.77 & 906.57 & 905.29 \\
\hline$I 22$ & 643.29 & 640.25 & 640.18 & 640.15 & 638.96 \\
\hline$I 23$ & 922.42 & 981.28 & 981.19 & 981.01 & 979.85 \\
\hline$I 31$ & 647.20 & 919.78 & 919.64 & 919.44 & 918.52 \\
\hline$I 32$ & 989.42 & 986.18 & 986.05 & 985.75 & 984.53 \\
\hline$I 33$ & 926.34 & 923.03 & 922.85 & 922.56 & 921.57 \\
\hline$I 41$ & 627.36 & 623.94 & 623.74 & 623.26 & 621.53 \\
\hline$I 42$ & 965.72 & 962.18 & 961.95 & 961.30 & 959.40 \\
\hline$I 43$ & 907.06 & 903.52 & 903.25 & 902.70 & 901.24 \\
\hline$P M 1$ & 339.03 & 334.57 & 334.46 & 333.64 & 316.85 \\
\hline$P M 2$ & 346.36 & 341.16 & 340.94 & 334.24 & 317.49 \\
\hline$P M 3$ & 349.88 & 342.68 & 342.55 & 334.96 & 318.00 \\
\hline$P M 4$ & 324.28 & 321.02 & 321.00 & 332.20 & 315.86 \\
\hline$P B 1$ & 317.21 & 316.70 & 316.79 & 316.81 & 317.23 \\
\hline$P B 2$ & 317.99 & 317.13 & 317.01 & 317.02 & 317.44 \\
\hline$P B 3$ & 317.33 & 316.75 & 316.75 & 316.77 & 317.21 \\
\hline$P B 4$ & 316.02 & 316.06 & 316.38 & 316.44 & 316.83 \\
\hline$P B 5$ & 324.12 & 321.51 & 321.84 & 321.85 & 322.16 \\
\hline
\end{tabular}


Table A.2.4: Calculated Errors for Output Temperatures of GaAs Model (Case - 2 in Table 4.5)

\begin{tabular}{|c|c|c|c|c|}
\hline Points & E_9ports & E_5ports & E_2ports & E_1port \\
\hline & & & & \\
\hline$I I I$ & 0.009323 & 0.009564 & 0.009776 & 0.014331 \\
\hline$I I 2$ & 0.004782 & 0.004946 & 0.005243 & 0.007672 \\
\hline$I I 3$ & 0.005326 & 0.005572 & 0.005900 & 0.007998 \\
\hline$I 2 I$ & 0.008855 & 0.009059 & 0.009147 & 0.012613 \\
\hline$I 22$ & 0.004588 & 0.004719 & 0.004982 & 0.006677 \\
\hline$I 23$ & 0.005137 & 0.005361 & 0.005682 & 0.007159 \\
\hline$I 31$ & 0.009073 & 0.009361 & 0.009735 & 0.013249 \\
\hline$I 32$ & 0.004700 & 0.004888 & 0.005323 & 0.007093 \\
\hline$I 33$ & 0.005285 & 0.005572 & 0.006035 & 0.007616 \\
\hline$I 41$ & 0.010447 & 0.011058 & 0.012524 & 0.017809 \\
\hline$I 42$ & 0.005318 & 0.005663 & 0.006639 & 0.009493 \\
\hline$I 43$ & 0.005831 & 0.006276 & 0.007182 & 0.009587 \\
\hline$P M 1$ & 0.114271 & 0.117089 & 0.138099 & 0.568281 \\
\hline$P M 2$ & 0.112166 & 0.116911 & 0.261432 & 0.622735 \\
\hline$P M 3$ & 0.144346 & 0.146953 & 0.299118 & 0.639134 \\
\hline$P M 4$ & 0.134267 & 0.135091 & 0.326194 & 0.346787 \\
\hline$P B 1$ & 0.029634 & 0.024404 & 0.023242 & 0.001162 \\
\hline$P B 2$ & 0.047804 & 0.054475 & 0.053919 & 0.030573 \\
\hline$P B 3$ & 0.033468 & 0.033468 & 0.032314 & 0.006924 \\
\hline$P B 4$ & 0.002497 & 0.022472 & 0.026217 & 0.050562 \\
\hline$P B 5$ & 0.108209 & 0.094527 & 0.094113 & 0.081260 \\
\hline
\end{tabular}

Table A.2.5: The Output Temperatures of GaAs Model (Case - 4 in Table 4.5)

\begin{tabular}{|c|c|c|c|c|c|}
\hline Points & T_detailed & T_9ports & T_5ports & T_2ports & T_1port \\
\hline & $\mathbf{( K )}$ & $\mathbf{( K )}$ & $\mathbf{( K )}$ & $\mathbf{( K )}$ & (K) \\
\hline$I I I$ & 673.69 & 668.11 & 666.71 & 666.18 & 665.19 \\
\hline$I I 2$ & 1013.61 & 1007.89 & 1006.44 & 1005.75 & 1004.66 \\
\hline$I 13$ & 952.49 & 946.69 & 945.21 & 944.59 & 943.79 \\
\hline$I 21$ & 684.16 & 679.32 & 678.39 & 678.19 & 677.46 \\
\hline$I 22$ & 1025.31 & 1020.37 & 1019.40 & 1019.06 & 1018.35 \\
\hline$I 23$ & 963.93 & 958.90 & 957.88 & 957.54 & 957.02 \\
\hline$I 31$ & 686.60 & 682.45 & 681.97 & 681.86 & 681.10 \\
\hline$I 32$ & 1028.84 & 1024.56 & 1024.04 & 1023.79 & 1023.02 \\
\hline$I 33$ & 965.80 & 961.47 & 960.91 & 960.64 & 960.06 \\
\hline$I 41$ & 664.72 & 661.03 & 661.07 & 661.20 & 660.03 \\
\hline$I 42$ & 1002.97 & 999.12 & 999.13 & 999.20 & 997.90 \\
\hline$I 43$ & 944.55 & 940.73 & 940.69 & 940.69 & 939.74 \\
\hline$P M 1$ & 384.84 & 377.76 & 376.01 & 368.62 & 355.35 \\
\hline$P M 2$ & 371.80 & 365.68 & 365.93 & 369.23 & 355.99 \\
\hline
\end{tabular}




\begin{tabular}{|c|c|c|c|c|c|}
\hline$P M 3$ & 388.84 & 380.06 & 379.22 & 369.90 & 356.50 \\
\hline$P M 4$ & 363.42 & 358.80 & 358.21 & 367.27 & 354.36 \\
\hline$P B 1$ & 362.81 & 357.49 & 355.24 & 355.17 & 355.73 \\
\hline$P B 2$ & 354.21 & 354.91 & 355.43 & 355.38 & 355.94 \\
\hline$P B 3$ & 360.31 & 356.45 & 355.20 & 355.13 & 355.70 \\
\hline$P B 4$ & 358.73 & 355.72 & 354.83 & 354.79 & 355.33 \\
\hline$P B 5$ & 367.38 & 361.59 & 360.27 & 360.19 & 360.65 \\
\hline
\end{tabular}

Table A.2.6: Calculated Errors for Output Temperatures of GaAs Model (Case - 4 in Table 4.5)

\begin{tabular}{|c|c|c|c|c|}
\hline Points & E 9ports & E 5ports & E_2ports & E_1port \\
\hline & & & & \\
\hline$I 1 / 1$ & 0.014932 & 0.018679 & 0.020097 & 0.022746 \\
\hline$I 13$ & 0.008016 & 0.010048 & 0.011014 & 0.012542 \\
\hline$I 21$ & 0.008889 & 0.011157 & 0.012107 & 0.013334 \\
\hline$I 22$ & 0.012599 & 0.015020 & 0.015540 & 0.017441 \\
\hline$I 23$ & 0.006811 & 0.008148 & 0.008617 & 0.009596 \\
\hline$I 31$ & 0.007576 & 0.009112 & 0.009625 & 0.010408 \\
\hline$I 32$ & 0.010735 & 0.011976 & 0.012261 & 0.014227 \\
\hline$I 33$ & 0.005872 & 0.006586 & 0.006929 & 0.007985 \\
\hline$I 41$ & 0.006503 & 0.007345 & 0.007750 & 0.008621 \\
\hline$I 42$ & 0.010117 & 0.010008 & 0.009651 & 0.012859 \\
\hline$I 43$ & 0.005477 & 0.005463 & 0.005363 & 0.007212 \\
\hline$P M 1$ & 0.005927 & 0.005989 & 0.005989 & 0.007463 \\
\hline$P M 2$ & 0.083451 & 0.104078 & 0.191183 & 0.347595 \\
\hline$P M 3$ & 0.085237 & 0.081755 & 0.035794 & 0.220195 \\
\hline$P M 4$ & 0.098829 & 0.108285 & 0.213192 & 0.364025 \\
\hline$P B 1$ & 0.072848 & 0.082151 & 0.060706 & 0.142857 \\
\hline$P B 2$ & 0.084700 & 0.120522 & 0.121637 & 0.112721 \\
\hline$P B 3$ & 0.012913 & 0.022505 & 0.021583 & 0.031913 \\
\hline$P B 4$ & 0.064003 & 0.084729 & 0.085890 & 0.076438 \\
\hline$P B 5$ & 0.051251 & 0.066406 & 0.067087 & 0.057892 \\
\hline & 0.085931 & 0.105521 & 0.106708 & 0.099881 \\
\hline
\end{tabular}


Table A.2.7: The Output Temperatures of GaAs Model (Case - 5 in Table 4.5)

\begin{tabular}{|c|c|c|c|c|c|}
\hline Points & T_detailed & T 9ports & T 5ports & T 2ports & T_1port \\
\hline & $(\mathbf{K})$ & $(\mathbf{K})$ & $(\mathbf{K})$ & $(\mathbf{K})$ & $(\mathbf{K})$ \\
\hline$I 11$ & 671.97 & 667.49 & 666.78 & 666.20 & 665.21 \\
\hline$I 12$ & 1012.79 & 1007.63 & 1006.59 & 1005.76 & 1004.68 \\
\hline$I I 3$ & 952.57 & 946.81 & 945.42 & 944.61 & 943.80 \\
\hline$I 21$ & 683.85 & 679.36 & 678.66 & 678.20 & 677.47 \\
\hline$I 22$ & 1025.94 & 1020.85 & 1019.79 & 1019.07 & 1018.36 \\
\hline$I 23$ & 965.42 & 959.72 & 958.29 & 957.55 & 957.04 \\
\hline$I 31$ & 687.79 & 683.19 & 682.45 & 681.87 & 681.12 \\
\hline$I 32$ & 1030.99 & 1025.77 & 1024.67 & 1023.80 & 1023.04 \\
\hline$I 33$ & 968.82 & 963.00 & 961.53 & 960.65 & 960.08 \\
\hline$I 41$ & 667.87 & 663.05 & 662.21 & 661.21 & 660.05 \\
\hline$I 42$ & 1007.17 & 1001.66 & 1000.50 & 999.21 & 997.92 \\
\hline$I 43$ & 949.43 & 943.35 & 941.85 & 940.70 & 939.75 \\
\hline$P M 1$ & 378.97 & 372.71 & 371.75 & 368.64 & 355.36 \\
\hline$P M 2$ & 386.48 & 379.40 & 378.28 & 369.25 & 356.00 \\
\hline$P M 3$ & 396.76 & 387.04 & 385.29 & 369.92 & 356.52 \\
\hline$P M 4$ & 351.76 & 347.76 & 348.46 & 367.29 & 354.37 \\
\hline$P B 1$ & 360.88 & 356.86 & 355.51 & 355.18 & 355.74 \\
\hline$P B 2$ & 361.77 & 357.30 & 355.73 & 355.39 & 355.95 \\
\hline$P B 3$ & 363.87 & 358.02 & 355.47 & 355.15 & 355.72 \\
\hline$P B 4$ & 352.71 & 354.25 & 355.07 & 354.80 & 355.34 \\
\hline$P B 5$ & 368.18 & 362.05 & 360.54 & 360.20 & 360.67 \\
\hline
\end{tabular}

Table A.2.8: Calculated Errors for Output Temperatures of GaAs Model (Case - 5 in Table 4.5)

\begin{tabular}{|c|c|c|c|c|}
\hline Points & E_9ports & E_5ports & E_2ports & E_1port \\
\hline$I I I$ & & & & \\
\hline$I I / 2$ & 0.012044 & 0.013953 & 0.015512 & 0.018174 \\
\hline$I 13$ & 0.007239 & 0.008698 & 0.009863 & 0.011378 \\
\hline$I 21$ & 0.008827 & 0.010957 & 0.012198 & 0.013439 \\
\hline$I 22$ & 0.011697 & 0.013521 & 0.014719 & 0.016621 \\
\hline$I 23$ & 0.007012 & 0.008472 & 0.009464 & 0.010442 \\
\hline$I 31$ & 0.008566 & 0.010715 & 0.011827 & 0.012594 \\
\hline$I 32$ & 0.011862 & 0.013770 & 0.015266 & 0.017200 \\
\hline$I 33$ & 0.007141 & 0.008646 & 0.009836 & 0.010876 \\
\hline$I 41$ & 0.008702 & 0.010900 & 0.012216 & 0.013068 \\
\hline$I 42$ & 0.013102 & 0.015386 & 0.018104 & 0.021258 \\
\hline$I 43$ & 0.007792 & 0.009432 & 0.011256 & 0.013080 \\
\hline$P M 1$ & 0.009362 & 0.011672 & 0.013443 & 0.014905 \\
\hline$P M 2$ & 0.079271 & 0.091427 & 0.130809 & 0.298974 \\
\hline$P M 3$ & 0.081869 & 0.094820 & 0.199237 & 0.352451 \\
\hline & 0.100455 & 0.118541 & 0.277387 & 0.415874 \\
\hline
\end{tabular}




\begin{tabular}{|c|c|c|c|c|}
\hline$P M 4$ & 0.077280 & 0.063756 & 0.300039 & 0.050425 \\
\hline$P B 1$ & 0.066032 & 0.088206 & 0.093627 & 0.084428 \\
\hline$P B 2$ & 0.072365 & 0.097782 & 0.103286 & 0.094220 \\
\hline$P B 3$ & 0.091592 & 0.131517 & 0.136527 & 0.127603 \\
\hline$P B 4$ & 0.029216 & 0.044773 & 0.039651 & 0.049896 \\
\hline$P B 5$ & 0.089909 & 0.112056 & 0.117043 & 0.110150 \\
\hline
\end{tabular}

Table A.2.9: The Output Temperatures of GaAs Model (Case - 6 in Table 4.5)

\begin{tabular}{|c|c|c|c|c|c|}
\hline Points & T_detailed & T_9ports & T_5ports & T_2ports & T_1port \\
\hline & $(\mathbf{K})$ & $\mathbf{( K )}$ & $\mathbf{( K )}$ & $\mathbf{( K )}$ & $\mathbf{( K )}$ \\
\hline$I 11$ & 672.01 & 667.14 & 666.23 & 666.20 & 665.21 \\
\hline$I 12$ & 1010.97 & 1006.47 & 1005.81 & 1005.76 & 1004.68 \\
\hline$I 13$ & 948.97 & 945.01 & 944.66 & 944.61 & 943.80 \\
\hline$I 22$ & 683.96 & 679.13 & 678.19 & 678.20 & 677.47 \\
\hline$I 23$ & 1024.22 & 1019.76 & 1019.08 & 1019.07 & 1018.36 \\
\hline$I 31$ & 661.91 & 957.93 & 957.58 & 957.55 & 957.04 \\
\hline$I 32$ & 1029.16 & 1024.57 & 1023.86 & 1023.80 & 1023.04 \\
\hline$I 33$ & 965.21 & 961.11 & 960.72 & 960.65 & 960.08 \\
\hline$I 41$ & 668.02 & 662.79 & 661.77 & 661.21 & 660.05 \\
\hline$I 42$ & 1005.53 & 1000.66 & 999.88 & 999.21 & 997.92 \\
\hline$I 43$ & 946.00 & 941.71 & 941.23 & 940.70 & 939.75 \\
\hline$P M 1$ & 377.93 & 372.11 & 371.41 & 368.64 & 355.36 \\
\hline$P M 2$ & 385.45 & 378.80 & 377.95 & 369.25 & 356.00 \\
\hline$P M 3$ & 372.90 & 363.93 & 363.85 & 369.92 & 356.52 \\
\hline$P M 4$ & 369.80 & 363.77 & 361.96 & 367.29 & 354.37 \\
\hline$P B 1$ & 356.85 & 355.95 & 355.25 & 355.18 & 355.74 \\
\hline$P B 2$ & 357.74 & 356.39 & 355.47 & 355.39 & 355.95 \\
\hline$P B 3$ & 353.50 & 354.58 & 355.21 & 355.15 & 355.72 \\
\hline$P B 4$ & 361.39 & 356.82 & 354.85 & 354.80 & 355.34 \\
\hline$P B 5$ & 364.15 & 360.90 & 360.27 & 360.20 & 360.67 \\
\hline
\end{tabular}


Table A.2.10: Calculated Errors for Output Temperatures of GaAs Model (Case - 6 in Table 4.5)

\begin{tabular}{|c|c|c|c|c|}
\hline Points & E_9ports & E_5ports & E_2ports & E_1port \\
\hline & & & & \\
\hline$I 11$ & 0.013091 & 0.015537 & 0.015618 & 0.018279 \\
\hline$I 12$ & 0.006329 & 0.007258 & 0.007328 & 0.008847 \\
\hline$I 2-1$ & 0.006102 & 0.006641 & 0.006718 & 0.007966 \\
\hline$I 22$ & 0.012579 & 0.015028 & 0.015002 & 0.016903 \\
\hline$I 23$ & 0.006158 & 0.007097 & 0.007111 & 0.008091 \\
\hline$I 31$ & 0.006013 & 0.006542 & 0.006587 & 0.007357 \\
\hline$I 32$ & 0.012763 & 0.015264 & 0.015368 & 0.017301 \\
\hline$I 33$ & 0.006295 & 0.007269 & 0.007351 & 0.008393 \\
\hline$I 41$ & 0.006163 & 0.006750 & 0.006855 & 0.007712 \\
\hline$I 42$ & 0.014211 & 0.016983 & 0.018504 & 0.021656 \\
\hline$I 43$ & 0.006903 & 0.008008 & 0.008958 & 0.010786 \\
\hline$P M 1$ & 0.006641 & 0.007384 & 0.008204 & 0.009675 \\
\hline$P M 2$ & 0.074682 & 0.083665 & 0.119210 & 0.289619 \\
\hline$P M 3$ & 0.077823 & 0.087771 & 0.189585 & 0.344646 \\
\hline$P M 4$ & 0.123045 & 0.124143 & 0.040878 & 0.224691 \\
\hline$P B 1$ & 0.086390 & 0.112321 & 0.035960 & 0.221060 \\
\hline$P B 2$ & 0.015831 & 0.028144 & 0.029376 & 0.019525 \\
\hline$P B 3$ & 0.023381 & 0.039314 & 0.040700 & 0.031001 \\
\hline$P B 4$ & 0.020187 & 0.031963 & 0.030841 & 0.041495 \\
\hline$P B 5$ & 0.074442 & 0.106532 & 0.107346 & 0.098550 \\
\hline & 0.050663 & 0.060483 & 0.061574 & 0.054248 \\
\hline
\end{tabular}




\section{A.3 Package Model}

Table A.3.1: Output Temperatures and Corresponding Errors of Package Model (Case - 1 in Table 4.9)

\begin{tabular}{|c|c|c|c|c|c|c|c|}
\hline Point & $T$ detail & T 3ports & E 3ports & T 2ports & E 2ports & T_1port & E_1port \\
\hline & $(\mathrm{K})$ & (K) & & $(\mathbf{K})$ & & (K) & \\
\hline S1 & 328.40 & 327.32 & 0.038028 & 327.47 & 0.032746 & 327.50 & 0.031690 \\
\hline$S 2$ & 329.36 & 327.98 & 0.047003 & 328.01 & 0.045981 & 328.03 & 0.045300 \\
\hline S3 & 329.72 & 328.13 & 0.053499 & 328.12 & 0.053836 & 328.13 & 0.053499 \\
\hline$S 4$ & 329.26 & 327.93 & 0.045455 & 327.97 & 0.044087 & 327.99 & 0.043404 \\
\hline S5 & 328.22 & 327.17 & 0.037208 & 327.33 & 0.031538 & 327.36 & 0.030475 \\
\hline S6 & 328.47 & 327.50 & 0.034071 & 327.55 & 0.032315 & 327.57 & 0.031612 \\
\hline S7 & 329.98 & 328.92 & 0.035357 & 328.22 & 0.058706 & 328.18 & 0.060040 \\
\hline$S 8$ & 330.67 & 329.25 & 0.046299 & 328.48 & 0.071405 & 328.30 & 0.077274 \\
\hline S9 & 329.79 & 328.83 & 0.032226 & 328.17 & 0.054381 & 328.14 & 0.055388 \\
\hline$S 10$ & 328.22 & 327.29 & 0.032955 & 327.38 & 0.029766 & 327.40 & 0.029057 \\
\hline$S 11$ & 327.80 & 327.19 & 0.021942 & 327.22 & 0.020863 & 327.23 & 0.020504 \\
\hline$S 12$ & 329.64 & 328.83 & 0.027328 & 328.07 & 0.052969 & 327.93 & 0.057692 \\
\hline$S 13$ & 330.55 & 329.75 & 0.02 & 329.06 & 0.048773 & 328.07 & 0.08 \\
\hline$S 14$ & 329.40 & 328.70 & 0.023810 & 327.97 & 0.048639 & 327.89 & 0.051361 \\
\hline$S 15$ & 327.53 & 326.95 & 0.021068 & 327.02 & 0.018525 & 327.03 & 0.018162 \\
\hline$S 16$ & 326.16 & 326.39 & 0.008792 & 326.46 & 0.011468 & 326.48 & 0.012232 \\
\hline$S 17$ & 327.63 & 327.90 & 0.009 & 327.28 & 2667 & 327.27 & 0.01 \\
\hline$S 18$ & 328.25 & 328.19 & 0.002124 & 327.49 & 0.026903 & 327.41 & 0.029735 \\
\hline$S 19$ & 327.46 & 327.81 & 0.012746 & 327.22 & 0.008740 & 327.22 & 0.008740 \\
\hline$S 20$ & 325.90 & 326.13 & 0.008880 & 326.23 & 0.012741 & 326.25 & 0.013514 \\
\hline$S 21$ & 323.59 & 4.39 & 0.03 & 324.55 & 695 & 324.58 & 0.0 \\
\hline$S 22$ & 324.62 & 325.26 & 0.025995 & 325.35 & 0.029651 & 325.38 & 0.030869 \\
\hline$S 23$ & 324.99 & 325.44 & 0.018007 & 325.51 & 0.020808 & 325.53 & 0.021609 \\
\hline$S 24$ & 324.52 & 325.20 & 0.027732 & 325.30 & 0.031811 & 325.32 & 0.032626 \\
\hline$S 25$ & 323.38 & 324.16 & 0.033362 & 324.32 & 0.040205 & 324.34 & 0.041061 \\
\hline$P 1$ & 339.99 & 339.13 & 0.02 & 338.76 & 0758 & 338.58 & 0.03 \\
\hline$P 2$ & 341.63 & 340.74 & 0.021379 & 340.35 & 0.030747 & 340.13 & 0.036032 \\
\hline$P 3$ & 341.63 & 340.74 & 0.021379 & 340.35 & 0.030747 & 340.13 & 0.036032 \\
\hline$P 4$ & 339.99 & 339.13 & 0.021505 & 338.76 & 0.030758 & 338.58 & 0.035259 \\
\hline$P 5$ & 341.51 & 340.74 & 0.01 & 340.35 & 7945 & 340.13 & 0.03 \\
\hline P6 & 346.04 & 345.25 & 0.017159 & 344.83 & 0.026281 & 344.56 & 0.032146 \\
\hline$P 7$ & 346.04 & 345.25 & 0.017159 & 344.83 & 0.026281 & 344.56 & 0.032146 \\
\hline$P 8$ & 341.51 & 340.74 & 0.018550 & 340.35 & 0.027945 & 340.13 & 0.033245 \\
\hline$P 9$ & 341.36 & 340.74 & 0.014990 & 340.35 & 0.024420 & 340.13 & 0.029739 \\
\hline$P 10$ & 345.89 & 345.25 & 0.013946 & 344.83 & 0.023099 & 344.56 & 0.028982 \\
\hline$P 11$ & 345.89 & 345.25 & 0.013946 & 344.83 & 0.023099 & 344.56 & 0.028982 \\
\hline$P 12$ & 341.36 & 340.74 & 0.014990 & 340.35 & 0.024420 & 340.13 & 0.029739 \\
\hline$P 13$ & 339.60 & 339.13 & 0.011869 & 338.76 & 0.021212 & 338.58 & 0.025758 \\
\hline P14 & 341.24 & 340.74 & 0.012124 & 340.35 & 0.021581 & 340.13 & 0.026916 \\
\hline
\end{tabular}




\begin{tabular}{|l|l|l|l|l|l|l|l|}
\hline$P 15$ & 341.24 & 340.74 & 0.012124 & 340.35 & 0.021581 & 340.13 & 0.026916 \\
\hline$P 16$ & 339.60 & 339.13 & 0.011869 & 338.76 & 0.021212 & 338.58 & 0.025758 \\
\hline
\end{tabular}

Table A.3.2: Output Temperatures and Corresponding Errors of Package Model (Case -2 in Table 4.9)

\begin{tabular}{|c|c|c|c|c|c|c|c|}
\hline Point & $\mathbf{T}$ detail & T 3ports & E 3ports & T 2ports & E 2ports & T 1port & E 1port \\
\hline & (K) & $(\mathbf{K})$ & & $(\mathbf{K})$ & & $(\mathbf{K})$ & \\
\hline S1 & 303.86 & 304.03 & 0.044041 & 304.21 & 0.090674 & 304.25 & 0.101036 \\
\hline$S 2$ & 304.51 & 304.52 & 0.002217 & 304.61 & 0.022173 & 304.65 & 0.031042 \\
\hline$S 3$ & 304.78 & 304.62 & 0.033473 & 304.67 & 0.023013 & 304.70 & 0.016736 \\
\hline$S 4$ & 304.44 & 304.49 & 0.011261 & 304.59 & 0.033784 & 304.63 & 0.042793 \\
\hline$S 5$ & 303.73 & 303.90 & 0.045576 & 304.08 & 0.093834 & 304.12 & 0.104558 \\
\hline S6 & 304.46 & 304.46 & 0.000000 & 304.57 & 0.024664 & 304.61 & 0.033632 \\
\hline$S 7$ & 305.63 & 305.56 & 0.012433 & 305.06 & 0.101243 & 305.04 & 0.104796 \\
\hline$S 8$ & 306.22 & 305.80 & 0.067524 & 305.24 & 0.157556 & 305.11 & 0.178457 \\
\hline 59 & 305.47 & 305.49 & 0.003656 & 305.02 & 0.082267 & 305.02 & 0.082267 \\
\hline$S 10$ & 304.28 & 304.29 & 0.002336 & 304.43 & 0.035047 & 304.47 & 0.044393 \\
\hline$S 11$ & 304.69 & 304.54 & 0.031 & 304.62 & 0.014925 & 304.66 & 0.00 \\
\hline$S 12$ & 306.12 & 305.74 & 0.062 & 305.20 & 0.150327 & 305.10 & 0.16 \\
\hline$S 13$ & 306.91 & 306.53 & 0.054993 & 306.05 & 0.124457 & 305.16 & 0.253256 \\
\hline 514 & 305.92 & 305.65 & 0.045608 & 305.13 & 0.133446 & 305.07 & 0.143581 \\
\hline$S 15$ & 304.49 & 304.36 & 0.028953 & 304 & 0.002227 & 304.52 & 0.006682 \\
\hline S16 & 304.34 & 30 & 0.01 & 304 & 0.0 & 304.56 & 0.05 \\
\hline$S 17$ & 305.35 & 305.42 & 0.013084 & 304.99 & 0.067290 & 305.00 & 0.065421 \\
\hline 518 & 305.84 & 305.60 & 0.041096 & 305.11 & 0.125000 & 305.06 & 0.133562 \\
\hline$S 19$ & 305.23 & 305.36 & 0.024857 & 304.96 & 0.051625 & 304.97 & 0.049713 \\
\hline$\$ 20$ & 304 & & 0.01 & 304 & 0.0 & 304.42 & 0.05 \\
\hline$S 21$ & 303.60 & 303.77 & 0.047222 & 303.95 & 0.097222 & 303.99 & 0.10 \\
\hline$S 22$ & 304.16 & 304.19 & 0.007212 & 304.33 & 0.040865 & 304.37 & 0.050481 \\
\hline$S 23$ & 304.39 & 304.27 & 0.027335 & 304.39 & 0.000000 & 304.43 & 0.009112 \\
\hline$S 24$ & & 304.16 & 0.01 & 304.31 & 0.048662 & 304.35 & 0.058394 \\
\hline$S 25$ & 303.49 & 303.65 & 0.045845 & 303.83 & 0.097421 & 303.87 & 0.10 \\
\hline$P 1$ & 316.70 & 316.48 & 0.013174 & 316.24 & 0.027545 & 316.09 & 0.036527 \\
\hline$P 2$ & 318.32 & 318.09 & 0.012555 & 317.83 & 0.026747 & 317.65 & 0.036572 \\
\hline$P 3$ & 318.32 & 318.09 & 0.012555 & 317.83 & 0.026747 & 317.65 & 0.036572 \\
\hline$P 4$ & 316.70 & 316.48 & 0.013174 & 316.24 & 0.027545 & 316.09 & 0.036527 \\
\hline P5 & 318.32 & 318.09 & 0.012555 & 317.82 & 0.027293 & 317.64 & 0.037118 \\
\hline$P 6$ & 322.84 & 322.59 & 0.010946 & 322.30 & 0.023643 & 322.08 & 0.033275 \\
\hline$P 7$ & 322.84 & 322.59 & 0.010946 & 322.30 & 0.023643 & 322.08 & 0.033275 \\
\hline$P 8$ & 318.32 & 318.09 & 0.012555 & 317.82 & 0.027293 & 317.64 & 0.037118 \\
\hline$P 9$ & 318.32 & 318.09 & 0.012555 & 317.82 & 0.027293 & 317.64 & 0.037118 \\
\hline$P 10$ & 322.84 & 322.59 & 0.010946 & 322.30 & 0.023643 & 322.08 & 0.033275 \\
\hline$P 11$ & 322.84 & 322.59 & 0.010946 & 322.30 & 0.023643 & 322.08 & 0.033275 \\
\hline P12 & 318.32 & 318.09 & 0.012555 & 317.82 & 0.027293 & 317.64 & 0.037118 \\
\hline
\end{tabular}




\begin{tabular}{|l|l|l|l|l|l|l|l|}
\hline$P 13$ & 316.70 & 316.48 & 0.013174 & 316.24 & 0.027545 & 316.09 & 0.036527 \\
\hline$P 14$ & 318.32 & 318.09 & 0.012555 & 317.83 & 0.026747 & 317.65 & 0.036572 \\
\hline$P 15$ & 318.32 & 318.09 & 0.012555 & 317.83 & 0.026747 & 317.65 & 0.036572 \\
\hline$P 16$ & 316.70 & 316.48 & 0.013174 & 316.24 & 0.027545 & 316.09 & 0.036527 \\
\hline
\end{tabular}

Table A.3.3: Output Temperatures and Corresponding Errors of Package Model (Case - 3 in Table 4.9)

\begin{tabular}{|c|c|c|c|c|c|c|c|}
\hline Point & $T_{\text {_ detail }}$ & T 3ports & E 3ports & T 2ports & E 2ports & T 1port & E_1port \\
\hline & (K) & $(\mathbf{K})$ & & (K) & & (K) & \\
\hline S1 & 301.79 & 301.83 & 0.022346 & 301.94 & 0.083799 & 302.00 & 0.117318 \\
\hline$S 2$ & 301.98 & 301.91 & 0.035354 & 301.99 & 0.005051 & 302.04 & 0.030303 \\
\hline S3 & 302.07 & 301.94 & 0.062802 & 302.00 & 0.033816 & 302.04 & 0.014493 \\
\hline$S 4$ & 301.95 & 301.91 & 0.020513 & 301.98 & 0.015385 & 302.03 & 0.041026 \\
\hline$S 5$ & 301.77 & 301.82 & 0.028249 & 301.93 & 0.090395 & 301.99 & 0.124294 \\
\hline S6 & 301.91 & 301.87 & 0.020942 & 301.95 & 0.020942 & 302.00 & 0.047120 \\
\hline$S 7$ & 302.28 & 302.22 & 0.026316 & 302.02 & 0.114035 & 302.05 & 0.100877 \\
\hline 58 & 302.50 & 302.30 & 0.080000 & 302.08 & 0.168000 & 302.06 & 0.176000 \\
\hline 59 & 302.22 & 302.19 & 0.013514 & 302.01 & 0.094595 & 302.04 & 0.081081 \\
\hline$S 10$ & 301.87 & 301.85 & 0.010695 & 301.94 & 0.037433 & 301.99 & 0.064171 \\
\hline$S 11$ & 301.92 & 301.86 & 0.031250 & 301.93 & 0.005208 & 301.98 & 0.03125 \\
\hline$S 12$ & 302.40 & 302 & 0.058333 & 302.04 & 0.150000 & 302.03 & 0.154167 \\
\hline$S 13$ & 302.73 & 302.59 & 0.051282 & 302.40 & 0.120879 & 302.04 & 0.252747 \\
\hline$S 14$ & 302.32 & 302.22 & 0.043103 & 302.02 & 0.129310 & 302.03 & 0.125000 \\
\hline$S 15$ & 301.87 & 301.83 & 0.021390 & 301.92 & 0.026738 & 301.96 & 0.048128 \\
\hline$S 16$ & 30 & 301 & 0.034 & 301.87 & 0.080925 & 301.92 & 0.109827 \\
\hline$S 17$ & 302.05 & 302.11 & 0.029268 & 301.95 & 0.04878 & 301.98 & 0.034146 \\
\hline$S 18$ & 302.23 & 302.18 & 0.022422 & 301.98 & 0.112108 & 301.99 & 0.107623 \\
\hline$S 19$ & 302.00 & 302.09 & 0.045000 & 301.94 & 0.030000 & 301.98 & 0.010000 \\
\hline$S 20$ & & 301.76 & 0.041420 & 301.86 & 0.100592 & 301.91 & 0.130178 \\
\hline$S 21$ & 301.46 & 301.62 & 0.109589 & 301.74 & 0.191781 & 301.79 & 0.226027 \\
\hline$S 22$ & 301.63 & 301.71 & 0.049080 & 301.80 & 0.104294 & 301.84 & 0.128834 \\
\hline 523 & 301.70 & 301.73 & 0.017647 & 301.81 & 0.064706 & 301.85 & 0.088235 \\
\hline$S 24$ & & 301.70 & 0.062500 & 301.79 & 0.118750 & 301.84 & 0.150000 \\
\hline$S 25$ & 301.43 & 301.61 & 0.125874 & 301.72 & 0.202797 & 301.77 & 0.237762 \\
\hline$P 1$ & 301.17 & 301.16 & 0.008547 & 301.13 & 0.034188 & 301.12 & 0.042735 \\
\hline$P 2$ & 302.26 & 302.23 & 0.013274 & 302.20 & 0.026549 & 302.19 & 0.030973 \\
\hline$P 3$ & 302.26 & 302.23 & 0.013274 & 302.20 & 0.026549 & 302.19 & 0.030973 \\
\hline$P 4$ & 301.17 & & 0.008547 & 301.13 & 0.034188 & 301.12 & 0.042735 \\
\hline$P 5$ & 302.26 & 302.23 & 0.013274 & 302.20 & 0.026549 & 302.19 & 0.030973 \\
\hline$P 6$ & 305.93 & 305.90 & 0.005059 & 305.87 & 0.010118 & 305.84 & 0.015177 \\
\hline P7 & 305.93 & 305.90 & 0.005059 & 305.87 & 0.010118 & 305.84 & 0.015177 \\
\hline$P 8$ & 302.26 & 302.23 & 0.013274 & 302.20 & 0.026549 & 302.19 & 0.030973 \\
\hline$P 9$ & 302.25 & 302.23 & 0.008889 & 302.20 & 0.022222 & 302.19 & 0.026667 \\
\hline$P 10$ & 305.93 & 305.90 & 0.005059 & 305.87 & 0.010118 & 305.84 & 0.015177 \\
\hline
\end{tabular}




\begin{tabular}{|l|l|l|l|l|l|l|l|}
\hline$P 11$ & 305.93 & 305.90 & 0.005059 & 305.87 & 0.010118 & 305.84 & 0.015177 \\
\hline$P 12$ & 302.25 & 302.23 & 0.008889 & 302.20 & 0.022222 & 302.19 & 0.026667 \\
\hline$P 13$ & 301.17 & 301.16 & 0.008547 & 301.13 & 0.034188 & 301.12 & 0.042735 \\
\hline$P 14$ & 302.25 & 302.23 & 0.008889 & 302.20 & 0.022222 & 302.19 & 0.026667 \\
\hline$P 15$ & 302.25 & 302.23 & 0.008889 & 302.20 & 0.022222 & 302.19 & 0.026667 \\
\hline$P 16$ & 301.17 & 301.16 & 0.008547 & 301.13 & 0.034188 & 301.12 & 0.042735 \\
\hline
\end{tabular}

Table A.3.4: Output Temperatures and Corresponding Errors of Package Model (Case -4 in Table 4.9)

\begin{tabular}{|c|c|c|c|c|c|c|c|}
\hline Point & T detail & T 3ports & E 3ports & T 2ports & E 2ports & T 1port & E 1port \\
\hline & (K) & $(\mathbf{K})$ & & (K) & & (K) & \\
\hline S1 & 300.47 & 300.56 & 0.191489 & 300.66 & 0.404255 & 300.69 & 0.468085 \\
\hline$S 2$ & 300.67 & 300.67 & 0.000000 & 300.73 & 0.089552 & 300.76 & 0.134328 \\
\hline$S 3$ & 300.76 & 300.70 & 0.078947 & 300.74 & 0.026316 & 300.77 & 0.013158 \\
\hline$S 4$ & 300.65 & 300.66 & 0.015385 & 300.72 & 0.107692 & 300.75 & 0.153846 \\
\hline S5 & 300.45 & 300.54 & 0.200000 & 300.64 & 0.422222 & 300.67 & 0.488889 \\
\hline$S 6$ & 300.66 & 300.65 & 0.015152 & 300.72 & 0.090909 & 300.75 & 0.136364 \\
\hline$S 7$ & 301.05 & 301.03 & 0.019048 & 300.81 & 0.228571 & 300.82 & 0.219048 \\
\hline$S 8$ & 301.29 & 301.12 & 0.131783 & 300 & 0.317829 & 300.83 & 0.356589 \\
\hline$S 9$ & 300.99 & 301.00 & 0.010101 & 300.80 & 0.191919 & 300.82 & 0.171717 \\
\hline 510 & 300.61 & 300.62 & 0.016393 & 300.70 & 0.147541 & 300.73 & 0.196721 \\
\hline S11 & 300.73 & 300.67 & 0.082192 & 300.73 & 0.000000 & 300.76 & 0.041096 \\
\hline$S 12$ & 301.25 & 301 & 0.12 & 300.86 & 0.312000 & 300.83 & 0.336000 \\
\hline$S 13$ & 301.60 & 301.45 & 0.093750 & 301.25 & 0.218750 & 300.84 & 0.475000 \\
\hline$S 14$ & 301.17 & 301.06 & 0.094017 & 300.84 & 0.282051 & 300.83 & 0.290598 \\
\hline$S 15$ & 300.68 & 300.64 & 0.058824 & 300.71 & 0.044118 & 300.74 & 0.088235 \\
\hline$S 16$ & 300 & 300 & 0.04 & 300 & 0.163934 & 300.74 & 3115 \\
\hline S17 & 300.95 & 300.98 & 0.03 & 300 & 0.157895 & 300.82 & 6842 \\
\hline$\$ 18$ & 301.14 & 301.04 & 0.087719 & 300.83 & 0.271930 & 300.83 & 0.271930 \\
\hline$S 19$ & 300.90 & 300.96 & 0.066667 & 300.79 & 0.122222 & 300.81 & 0.100000 \\
\hline$\$ 20$ & 300.57 & 300.61 & 0.070175 & & 0.210526 & & 0.263158 \\
\hline$S 21$ & 300.42 & 300 & 0.23 & 300 & 0.47 & 30 & 7619 \\
\hline$S 22$ & 300.58 & 300.60 & 0.034483 & 300.68 & 0.172414 & 300.71 & 0.224138 \\
\hline$S 23$ & 300.66 & 300.62 & 0.060606 & 300.69 & 0.045455 & 300.72 & 0.090909 \\
\hline$S 24$ & 300.56 & 300.60 & 0.071429 & 300.68 & 0.214286 & 300.71 & 0.267857 \\
\hline$S 25$ & 300.40 & 300.50 & 0.250000 & 300.69 & 0.500000 & & 0.575000 \\
\hline$P 1$ & 301.06 & 301.05 & 0.009434 & 301.02 & 0.037736 & 301.01 & 0.047170 \\
\hline$P 2$ & 302.14 & 302.12 & 0.009346 & 302.08 & 0.028037 & 302.07 & 0.032710 \\
\hline$P 3$ & 302.14 & 302.12 & 0.009346 & 302.08 & 0.028037 & 302.07 & 0.032710 \\
\hline$P 4$ & 301.06 & 301.05 & 0.009434 & 301.02 & 0.037736 & 301.01 & 0.047170 \\
\hline$P 5$ & 302.14 & 302.12 & 0.009346 & 302.08 & 0.028037 & 302.07 & 0.032710 \\
\hline P6 & 305.81 & 305.78 & 0.005164 & 305.74 & 0.012048 & 305.71 & 0.017212 \\
\hline$P 7$ & 305.81 & 305.78 & 0.005164 & 305.74 & 0.012048 & 305.71 & 0.017212 \\
\hline$P 8$ & 302.14 & 302.12 & 0.009346 & 302.08 & 0.028037 & 302.07 & 0.032710 \\
\hline
\end{tabular}




\begin{tabular}{|c|c|c|c|c|c|c|c|}
\hline$P 9$ & 302.14 & 302.12 & 0.009346 & 302.08 & 0.028037 & 302.07 & 0.032710 \\
\hline$P 10$ & 305.81 & 305.78 & 0.005164 & 305.74 & 0.012048 & 305.71 & 0.017212 \\
\hline$P 11$ & 305.81 & 305.78 & 0.005164 & 305.74 & 0.012048 & 305.71 & 0.017212 \\
\hline$P 12$ & 302.14 & 302.12 & 0.009346 & 302.08 & 0.028037 & 302.07 & 0.032710 \\
\hline$P 13$ & 301.06 & 301.05 & 0.009434 & 301.02 & 0.037736 & 301.01 & 0.047170 \\
\hline$P 14$ & 302.14 & 302.12 & 0.009346 & 302.08 & 0.028037 & 302.07 & 0.032710 \\
\hline$P 15$ & 302.14 & 302.12 & 0.009346 & 302.08 & 0.028037 & 302.07 & 0.032710 \\
\hline$P 16$ & 301.06 & 301.05 & 0.009434 & 301.02 & 0.037736 & 301.01 & 0.047170 \\
\hline
\end{tabular}

Table A.3.5: Output Temperatures and Corresponding Errors of Package Model (Case - 6 in Table 4.9)

\begin{tabular}{|c|c|c|c|c|c|c|c|}
\hline Point & $T_{\text {_ detail }}$ & T_3ports & E 3ports & T_2ports & E 2ports & $T$ lport & E_1port \\
\hline & (K) & (K) & & (K) & & (K) & \\
\hline S1 & 300.48 & 300.57 & 0.187500 & 300.67 & 0.395833 & 300.71 & 0.479167 \\
\hline$S 2$ & 300.68 & 300.68 & 0.000000 & 300.74 & 0.088235 & 300.77 & 0.132353 \\
\hline$S 3$ & 300.78 & 300.71 & 0.089744 & 300.75 & 0.038462 & 300.78 & 0.000000 \\
\hline$S 4$ & 300.66 & 300.67 & 0.015152 & 300.73 & 0.106061 & 300.77 & 0.166667 \\
\hline S5 & 300.45 & 300.55 & 0.222222 & 300.65 & 0.444444 & 300.68 & 0.511111 \\
\hline S6 & 300.67 & 300.67 & 0.000000 & 300.73 & 0.089552 & 300.76 & 0.134328 \\
\hline$S 7$ & 301.07 & 301.05 & 0.018692 & 300.83 & 0.224299 & 300.84 & 0.214953 \\
\hline 58 & 301.31 & 301.14 & 0.129771 & 300.90 & 0.312977 & 300.85 & 0.351145 \\
\hline$S 9$ & 301.01 & 301.02 & 0.009901 & 300.82 & 0.188119 & 300.83 & 0.178218 \\
\hline$S 10$ & 300.62 & 300.63 & 0.016129 & 300.71 & 0.145161 & 300.74 & 0.193548 \\
\hline$S 11$ & 300.75 & 300.69 & 0.080000 & 300.74 & 0.013333 & 300.77 & 0.026667 \\
\hline$S 12$ & 301.27 & 301.12 & 0.118110 & 300.88 & 0.307087 & 300.85 & 0.330709 \\
\hline$S 13$ & 301.63 & 301.47 & 0.098160 & 301.27 & 0.220859 & 300.86 & 0.472393 \\
\hline$S 14$ & 301.19 & 301.08 & 0.092437 & 300.85 & 0.285714 & 300.84 & 0.294118 \\
\hline$S 15$ & 300.69 & 300.65 & 0.057971 & 300.72 & 0.043478 & 300.75 & 0.086957 \\
\hline 516 & 300.63 & 300.65 & 0.031746 & 300.72 & 0.142857 & 300.76 & 0.206349 \\
\hline$S 17$ & 300.96 & 301.00 & 0.041667 & 300.81 & 0.156250 & 300.83 & 0.135417 \\
\hline$S 18$ & 301.15 & 301.06 & 0.078261 & 300.85 & 0.260870 & 300.84 & 0.269565 \\
\hline$S 19$ & 300.92 & 300.98 & 0.065217 & 300.80 & 0.130435 & 300.83 & 0.097826 \\
\hline$S 20$ & 300.58 & 300.62 & 0.068966 & 300.70 & 0.206897 & 300.73 & 0.258621 \\
\hline$S 21$ & 300.43 & 300.53 & 0.232558 & 300.63 & 0.465116 & 300.66 & 0.534884 \\
\hline$S 22$ & 300.60 & 300.61 & 0.016667 & 300.69 & 0.150000 & 300.72 & 0.200000 \\
\hline$S 23$ & 300.67 & 300.63 & 0.059701 & 300.70 & 0.044776 & 300.73 & 0.089552 \\
\hline$S 24$ & 300.57 & 300.61 & 0.070175 & 300.69 & 0.210526 & 300.72 & 0.263158 \\
\hline$S 25$ & 300.41 & 300.51 & 0.243902 & 300.61 & 0.487805 & 300.64 & 0.560976 \\
\hline$P 1$ & 301.12 & 301.10 & 0.017857 & 301.07 & 0.044643 & 301.06 & 0.053571 \\
\hline$P 2$ & 302.23 & 302.21 & 0.008969 & 302.18 & 0.022422 & 302.16 & 0.031390 \\
\hline$P 3$ & 302.23 & 302.21 & 0.008969 & 302.18 & 0.022422 & 302.16 & 0.031390 \\
\hline$P 4$ & 301.12 & 301.10 & 0.017857 & 301.07 & 0.044643 & 301.06 & 0.053571 \\
\hline$P 5$ & 302.23 & 302.21 & 0.008969 & 302.18 & 0.022422 & 302.16 & 0.031390 \\
\hline$P 6$ & 305.98 & 305.95 & 0.005017 & 305.91 & 0.011706 & 305.88 & 0.016722 \\
\hline
\end{tabular}




\begin{tabular}{|c|c|c|c|c|c|c|c|}
\hline$P 7$ & 305.98 & 305.95 & 0.005017 & 305.91 & 0.011706 & 305.88 & 0.016722 \\
\hline$P 8$ & 302.23 & 302.21 & 0.008969 & 302.18 & 0.022422 & 302.16 & 0.031390 \\
\hline$P 9$ & 302.23 & 302.21 & 0.008969 & 302.18 & 0.022422 & 302.16 & 0.031390 \\
\hline$P 10$ & 305.98 & 305.95 & 0.005017 & 305.91 & 0.011706 & 305.88 & 0.016722 \\
\hline$P 11$ & 305.98 & 305.95 & 0.005017 & 305.91 & 0.011706 & 305.88 & 0.016722 \\
\hline$P 12$ & 302.23 & 302.21 & 0.008969 & 302.18 & 0.022422 & 302.16 & 0.031390 \\
\hline$P 13$ & 301.12 & 301.10 & 0.017857 & 301.07 & 0.044643 & 301.06 & 0.053571 \\
\hline$P 14$ & 302.23 & 302.21 & 0.008969 & 302.18 & 0.022422 & 302.16 & 0.031390 \\
\hline$P 15$ & 302.23 & 302.21 & 0.008969 & 302.18 & 0.022422 & 302.16 & 0.031390 \\
\hline$P 16$ & 301.12 & 301.10 & 0.017857 & 301.07 & 0.044643 & 301.06 & 0.053571 \\
\hline
\end{tabular}




\section{Appendix B}

\section{Neural Network Modeling Results}

\section{B.1 GaN Based Power Package Amplifier}

Table B.1.1: Testing Errors of GaN Model with Four-Layer Perceptrons and FourPort Configuration

\begin{tabular}{|c|c|c|c|c|}
\hline Output & $\begin{array}{l}\text { Average Error } \\
\text { (BP4) }\end{array}$ & $\begin{array}{l}\text { Maximum Error } \\
\text { (BP4) }\end{array}$ & $\begin{array}{l}\text { Average Error } \\
\text { (CG4) }\end{array}$ & $\begin{array}{c}\text { Maximum Error } \\
\text { (CG4) }\end{array}$ \\
\hline$T$-linear & 2.41494270 & 20.0477350 & 2.74927880 & 21.1156850 \\
\hline$T$-nonlinear & 2.65020900 & 18.2094290 & 2.97454670 & 19.2585770 \\
\hline$T$-bot & 0.94954900 & 3.7385294 & 2.44730760 & 8.3350440 \\
\hline$T M 1$ & 11.84090300 & 27.8511410 & 11.87790800 & 27.8989430 \\
\hline$T M 2$ & 11.76631600 & 28.5727310 & 11.39116200 & 30.6001680 \\
\hline$T M 3$ & 1.95915210 & 8.4325040 & 1.89793680 & 7.8421397 \\
\hline$I 1-1$ & 0.55015240 & 3.5540729 & 0.71823370 & 5.2614713 \\
\hline$I 1-2$ & 0.82111520 & 3.2995460 & 0.92921525 & 4.9944067 \\
\hline$I 1-3$ & 1.12536860 & 3.8178330 & 1.20561900 & 5.4506120 \\
\hline$I 1-4$ & 0.77888000 & 3.6058020 & 0.93492573 & 5.7536700 \\
\hline$I 1-5$ & 0.79244184 & 2.9692955 & 0.90955615 & 4.8699190 \\
\hline$I 1-6$ & 1.29211710 & 4.0354857 & 1.36314680 & 5.9223000 \\
\hline$I 2-1$ & 1.14966820 & 4.2125044 & 1.27807100 & 6.4492683 \\
\hline $12-2$ & 0.73016196 & 2.8608134 & 0.86132700 & 4.7709904 \\
\hline $12-3$ & 1.54429610 & 4.8665504 & 1.59063300 & 6.3749814 \\
\hline$I 2-4$ & 1.08311500 & 4.0398355 & 1.21692650 & 6.3140440 \\
\hline$I 2-5$ & 0.71082410 & 2.8319552 & 0.84514105 & 4.7983130 \\
\hline $12-6$ & 1.37532290 & 4.3464127 & 1.43168400 & 6.0881110 \\
\hline $13-1$ & 0.71708393 & 3.4185455 & 0.88327280 & 5.6088140 \\
\hline $13-2$ & 0.85064775 & 3.0549430 & 0.95585470 & 4.9841300 \\
\hline $13-3$ & 1.11937710 & 3.6118440 & 1.19530930 & 5.4427986 \\
\hline $13-4$ & 0.55984664 & 3.4678640 & 0.72927964 & 5.2841650 \\
\hline $13-5$ & 0.87061197 & 3.3997302 & 0.96980494 & 5.1098290 \\
\hline $13-6$ & 1.05676910 & 3.8432431 & 1.12704460 & 5.2571970 \\
\hline
\end{tabular}




\begin{tabular}{|c|c|c|c|c|}
\hline$P M 1$ & 22.1079180 & 50.7927470 & 22.10477300 & 50.4745980 \\
\hline$P M 2$ & 26.0629180 & 63.4717700 & 25.22136100 & 66.6689400 \\
\hline$P M 3$ & 1.2235150 & 6.7541830 & 5.64201160 & 19.0049860 \\
\hline$P B 1$ & 1.3535178 & 7.2056375 & 1.23957540 & 6.2986856 \\
\hline$P B 2$ & 2.0426793 & 8.5403000 & 1.89845920 & 7.4756690 \\
\hline$P B 3$ & 1.3529834 & 7.2141604 & 1.24716650 & 6.4967990 \\
\hline$P B 4$ & 1.7862096 & 8.3772350 & 1.65786610 & 7.3197374 \\
\hline$P 1$ & 0.8424973 & 3.3342156 & 0.94690907 & 4.9791230 \\
\hline$P 2$ & 0.8154406 & 3.0321610 & 0.92898190 & 4.8494220 \\
\hline$P 3$ & 0.7564825 & 2.9488292 & 0.88277006 & 4.7466050 \\
\hline$P 4$ & 0.7379744 & 2.9195542 & 0.86651890 & 4.7765430 \\
\hline$P 5$ & 0.8710570 & 3.0985190 & 0.97342100 & 4.9700994 \\
\hline$P 6$ & 0.8895933 & 3.4330437 & 0.98566705 & 5.0985017 \\
\hline$P 7$ & 1.5432045 & 6.0184474 & 1.54196320 & 6.8637147 \\
\hline$P 8$ & 2.3006756 & 7.1425050 & 2.31404570 & 8.1457290 \\
\hline$P 9$ & 3.3399673 & 9.3369130 & 3.36002420 & 9.8615800 \\
\hline$P 10$ & 2.8951232 & 8.1873820 & 2.93542900 & 9.1361010 \\
\hline$P 11$ & 2.0703588 & 6.6060624 & 2.09078030 & 7.7820625 \\
\hline$P 12$ & 1.1547043 & 4.9673710 & 1.19888730 & 6.2476754 \\
\hline
\end{tabular}


Table B.1.2: Testing Errors of GaN Model with Four-Layer Perceptrons \& FivePort Configuration

\begin{tabular}{|c|c|c|c|c|}
\hline Output & $\begin{array}{c}\text { Average Error } \\
\text { (BP4) }\end{array}$ & $\begin{array}{c}\text { Maximum Error } \\
\text { (BP4) }\end{array}$ & $\begin{array}{l}\text { Average Error } \\
\text { (CG4) }\end{array}$ & $\begin{array}{l}\text { Maximum Error } \\
\text { (CG4) }\end{array}$ \\
\hline T-linear & 2.17259170 & 10.4746650 & 3.36802740 & 15.3559350 \\
\hline T-nonlinear & 2.23280050 & 6.8196445 & 2.73128370 & 9.7784440 \\
\hline$T P I$ & 15.68848500 & 47.4356460 & 10.15829850 & 28.7971420 \\
\hline$T P 2$ & 16.12359000 & 54.4901540 & 16.96480000 & 54.7249450 \\
\hline$T P 3$ & 16.11911600 & 50.5476400 & 15.47529000 & 46.2334900 \\
\hline$T P 4$ & 15.64284800 & 48.9603300 & 15.95302500 & 46.5533180 \\
\hline$T P 5$ & 15.56838600 & 52.6432270 & 14.28571800 & 49.8728070 \\
\hline$I 1-1$ & 1.46619470 & 4.5749187 & 1.29440140 & 4.0734740 \\
\hline$I 1-2$ & 1.22218610 & 4.3127890 & 0.88989896 & 3.4562707 \\
\hline$I 1-3$ & 1.62009690 & 4.0859175 & 1.28243770 & 4.0851064 \\
\hline$I I-4$ & 1.06415340 & 2.9429991 & 0.92463225 & 3.2941809 \\
\hline$I 1-5$ & 0.90999925 & 3.3210523 & 0.72675460 & 2.7610285 \\
\hline$I 1-6$ & 1.18584600 & 3.2591925 & 1.02044560 & 3.2749126 \\
\hline$I 2-1$ & 0.60871905 & 1.7220162 & 0.48107030 & 2.2384927 \\
\hline$I 2-2$ & 0.54866390 & 1.8449856 & 0.49301913 & 1.7890356 \\
\hline$I 2-3$ & 0.72768843 & 2.6961780 & 0.69435316 & 2.4959285 \\
\hline$I 2-4$ & 0.71020940 & 2.1779760 & 0.62730306 & 2.5150812 \\
\hline$I 2-5$ & 0.46779700 & 1.8975588 & 0.50385070 & 1.9993135 \\
\hline$I 2-6$ & 0.85374230 & 2.7876210 & 0.78158400 & 2.5681038 \\
\hline $13-1$ & 1.12040750 & 3.1508274 & 1.06948580 & 3.7246535 \\
\hline$I 3-2$ & 0.68799590 & 2.9099405 & 0.74499416 & 3.2217060 \\
\hline$I 3-3$ & 1.23644240 & 3.2575107 & 1.17494920 & 3.6941495 \\
\hline $13-4$ & 1.42150500 & 3.9602976 & 1.37938010 & 4.4670315 \\
\hline$I 3-5$ & 0.90723044 & 3.6529431 & 0.95050800 & 4.0303874 \\
\hline$I 3-6$ & 1.53699760 & 4.0735180 & 1.49121930 & 4.6010056 \\
\hline$P M 1$ & 3.19889070 & 10.5147660 & 2.02670980 & 6.2983740 \\
\hline$P M 2$ & 3.66192530 & 11.7894600 & 2.36231760 & 6.7698207 \\
\hline$P M 3$ & 4.26113270 & 13.7086100 & 2.74282240 & 7.8856044 \\
\hline$P B 1$ & 2.84373550 & 7.6566670 & 2.61803960 & 7.5139090 \\
\hline$P B 2$ & 3.08080940 & 8.2615140 & 2.11776450 & 6.4456644 \\
\hline$P B 3$ & 2.91596560 & 7.5821195 & 2.79714350 & 8.5284150 \\
\hline$P B 4$ & 3.19786520 & 8.6553660 & 3.15176000 & 7.9516654 \\
\hline$P 1$ & 1.23602460 & 4.2311673 & 0.88987750 & 3.4273713 \\
\hline$P 2$ & 0.92473406 & 3.2749430 & 0.73280185 & 2.7381775 \\
\hline$P 3$ & 0.56838936 & 1.8609947 & 0.50652117 & 1.8394618 \\
\hline$P 4$ & 0.48314282 & 1.9559169 & 0.51565546 & 2.0566500 \\
\hline$P 5$ & 0.69871450 & 2.9667760 & 0.75307447 & 3.2853363 \\
\hline$P 6$ & 0.91390866 & 3.7119002 & 0.95726687 & 4.0995740 \\
\hline$P 7$ & 1.70691470 & 4.3119802 & 1.45258980 & 4.3540440 \\
\hline$P 8$ & 1.31731240 & 3.3096933 & 1.05085900 & 3.3261073 \\
\hline$P 9$ & 0.95425140 & 3.1144874 & 0.63327070 & 2.1721065 \\
\hline$P 10$ & 1.00033970 & 3.5282270 & 0.78455186 & 3.2008817 \\
\hline$P 11$ & 1.38839880 & 4.0384936 & 1.26517620 & 4.3716360 \\
\hline$P 12$ & 1.64607920 & 4.6116510 & 1.56268850 & 5.0012390 \\
\hline
\end{tabular}




\section{B.2 GaAs Based Power Amplifier}

Table B.2.1: Testing Errors of GaAs Model with Four-Layer Perceptrons and FivePort Configuration

\begin{tabular}{|c|c|c|c|c|}
\hline Output & $\begin{array}{c}\text { Average Error } \\
\text { (BP4) }\end{array}$ & $\begin{array}{c}\text { Maximum } \\
\text { Error (BP4) }\end{array}$ & $\begin{array}{l}\text { Average Error } \\
\text { (CG4) }\end{array}$ & $\begin{array}{c}\text { Maximum } \\
\text { Error (CG4) }\end{array}$ \\
\hline T-linear & 3.14161100 & 12.7752970 & 2.8381526 & 12.8778640 \\
\hline T-nonlinear & 3.22117140 & 12.9597150 & 3.0847550 & 11.9766290 \\
\hline$T P 1$ & 2.23572500 & 8.1451520 & 1.9249161 & 7.1759305 \\
\hline$T P 2$ & 12.99845300 & 28.7106420 & 7.9454346 & 26.8586600 \\
\hline TP3 & 13.16460800 & 29.6669730 & 13.0863490 & 30.9459340 \\
\hline TP4 & 2.40119120 & 7.3238125 & 10.7506270 & 33.1121940 \\
\hline TP5 & 11.97915200 & 28.4870300 & 12.1319820 & 27.7803970 \\
\hline$I 1-1$ & 0.96100026 & 3.2966511 & 1.3153844 & 4.6668644 \\
\hline $11-2$ & 0.61649360 & 2.1245070 & 1.1982026 & 3.3512020 \\
\hline$I 1-3$ & 0.60789880 & 2.1346533 & 1.1922393 & 3.4988377 \\
\hline$I 2-1$ & 0.74553720 & 2.6928590 & 1.1915933 & 3.9145155 \\
\hline$I 2-2$ & 0.60211325 & 2.3265480 & 1.2062361 & 3.7471542 \\
\hline$I 2-3$ & 0.59751326 & 2.2369206 & 1.1994054 & 3.7606764 \\
\hline I3-1 & 0.74498590 & 2.7463020 & 1.1856592 & 3.7589402 \\
\hline$I 3-2$ & 0.58735293 & 2.1959739 & 1.2184523 & 3.8083947 \\
\hline I3-3 & 0.58047634 & 2.1017864 & 1.2094997 & 3.8660808 \\
\hline I4-1 & 1.12255480 & 3.7394803 & 1.2359029 & 4.6055670 \\
\hline I4-2 & 0.61403920 & 2.2267058 & 1.2881348 & 4.5757550 \\
\hline I4-3 & 0.58971780 & 2.0164473 & 1.2641913 & 4.5097460 \\
\hline$P M 1$ & 23.86480300 & 49.6680100 & 23.5712130 & 54.7804450 \\
\hline$P M 2$ & 23.90364800 & 50.5984400 & 13.8245000 & 45.5214730 \\
\hline$P M 3$ & 23.72596000 & 55.1201900 & 23.9918960 & 53.4846700 \\
\hline$P M 4$ & 4.40305470 & 10.91938900 & 17.8149950 & 53.6402100 \\
\hline$P B 1$ & 2.14039470 & 7.76017900 & 1.9898176 & 6.7918990 \\
\hline$P B 2$ & 2.13810940 & 7.77460240 & 1.9624932 & 6.5614830 \\
\hline$P B 3$ & 2.13170770 & 7.72975540 & 1.9806156 & 6.7077427 \\
\hline$P B 4$ & 2.13170770 & 7.72975540 & 1.9806156 & 6.7077427 \\
\hline$P B 5$ & 2.11726570 & 7.79395150 & 1.9517250 & 6.6710534 \\
\hline
\end{tabular}




\section{B.3 Package Model}

Table B.3.1: Testing Errors of Package Model with Four-Layer Perceptrons and Three-Port Configuration

\begin{tabular}{|c|c|c|c|c|}
\hline Output & $\begin{array}{c}\text { Average Error } \\
\text { (BP4) }\end{array}$ & $\begin{array}{c}\text { Maximum } \\
\text { Error (BP4) }\end{array}$ & $\begin{array}{c}\text { Average Error } \\
\text { (CG4) }\end{array}$ & $\begin{array}{c}\text { Maximum } \\
\text { Error (CG4) }\end{array}$ \\
\hline$T$-linear & 6.08212500 & 20.5837300 & 5.9765690 & 20.2582700 \\
\hline$T$-nonlinear & 5.93204900 & 19.9994100 & 5.7717090 & 20.3551400 \\
\hline$T$-alpha & 3.90680000 & 12.2270500 & 3.4117790 & 13.0916800 \\
\hline$T P 1$ & 3.49536700 & 12.2769100 & 3.7540260 & 13.2647700 \\
\hline$T P 2$ & 2.85086900 & 9.0309170 & 2.8355440 & 9.9687490 \\
\hline$T P 3$ & 9.58026700 & 26.2361700 & 10.0311200 & 28.0604300 \\
\hline$P 1$ & 1.55512600 & 6.8407360 & 1.8787410 & 8.1925670 \\
\hline$P 2$ & 0.63569500 & 3.4298500 & 1.3152470 & 5.7767620 \\
\hline$P 3$ & 0.63569500 & 3.4298500 & 1.3152430 & 5.7766990 \\
\hline$P 4$ & 1.55512600 & 6.8407360 & 1.8787410 & 8.1925670 \\
\hline$P 5$ & 0.63617000 & 3.4321910 & 1.3153960 & 5.7784450 \\
\hline$P 6$ & 2.60626300 & 9.6395660 & 2.8474950 & 8.8371390 \\
\hline$P 7$ & 2.60625800 & 9.6395150 & 2.8474980 & 8.8371390 \\
\hline$P 8$ & 0.63614500 & 3.4320650 & 1.3153970 & 5.7784450 \\
\hline$P 9$ & 0.63614500 & 3.4320650 & 1.3153960 & 5.7784450 \\
\hline$P 10$ & 2.60625500 & 9.6395150 & 2.8474950 & 8.8371390 \\
\hline$P 11$ & 2.60625800 & 9.6395150 & 2.8474950 & 8.8371390 \\
\hline$P 12$ & 0.63614400 & 3.4320650 & 1.3153970 & 5.7784450 \\
\hline$P 13$ & 1.55512600 & 68407360 & 1.8787414 & 81925670 \\
\hline$P 14$ & 0.63569325 & 3.4298500 & 1.3152477 & 5.7767615 \\
\hline$P 15$ & 0.63571980 & 3.4299757 & 1.3152477 & 5.7767615 \\
\hline$P 16$ & 1.55512640 & 6.840736 & 1.8787410 & 8.1925670 \\
\hline
\end{tabular}




\section{Appendix C}

\section{Iteration Results}

\section{C.1 GaN Based Power Package Amplifier}

Table C.1.1: Output Temperatures for Detailed and Sub-models 3 and 4 and Corresponding Errors of GaN Model with Four-Port Configuration

\begin{tabular}{|c|c|c|c|c|c|c|}
\hline & \multicolumn{3}{|c|}{ Model-3 } & \multicolumn{3}{c|}{ Model-4 } \\
\hline Output & T full & T_sub & Error & T_full & T_sub & Error \\
\hline & $(\mathbf{K})$ & $\mathbf{( K )}$ & & $(\mathbf{K})$ & $(\mathbf{K})$ & \\
\hline$I-\max$ & 401.270520 & 403.1878 & 0.018932 & 401.444803 & 403.4073 & 0.019345 \\
\hline$I 1-1$ & 372.042118 & 374.2411 & 0.030524 & 376.295085 & 374.4163 & 0.024625 \\
\hline$I 1-2$ & 376.208840 & 381.4057 & 0.068192 & 380.456404 & 381.5867 & 0.014049 \\
\hline$I 1-3$ & 372.640068 & 375.4317 & 0.038431 & 376.698848 & 375.6148 & 0.014134 \\
\hline$I 1-4$ & 376.247013 & 377.8282 & 0.020738 & 376.427219 & 378.0137 & 0.020758 \\
\hline$I 1-5$ & 381.922821 & 386.5248 & 0.056175 & 382.103078 & 386.7191 & 0.056222 \\
\hline$I 1-6$ & 377.075942 & 379.1942 & 0.027483 & 377.248923 & 379.3813 & 0.027604 \\
\hline$I 2-1$ & 381.709384 & 378.7106 & 0.036701 & 381.886776 & 378.8956 & 0.036528 \\
\hline$I 2-2$ & 387.937108 & 388.4081 & 0.005356 & 388.114267 & 388.6069 & 0.005591 \\
\hline$I 2-3$ & 382.292999 & 379.9113 & 0.028942 & 382.462221 & 380.0875 & 0.028798 \\
\hline$I 2-4$ & 382.061111 & 379.7025 & 0.028742 & 382.233366 & 379.8846 & 0.028562 \\
\hline$I 2-5$ & 388.322290 & 386.4126 & 0.021622 & 388.494058 & 386.6010 & 0.021392 \\
\hline$I 2-6$ & 382.735802 & 379.9765 & 0.033351 & 382.900077 & 380.1559 & 0.033102 \\
\hline$I 3-1$ & 381.264865 & 378.5138 & 0.033853 & 377.666157 & 378.6947 & 0.013243 \\
\hline$I 3-2$ & 387.010081 & 386.7947 & 0.002475 & 383.576467 & 386.9750 & 0.040664 \\
\hline$I 3-3$ & 381.873262 & 380.1200 & 0.021414 & 378.533109 & 380.3088 & 0.022611 \\
\hline$I 3-4$ & 374.743101 & 376.5119 & 0.023665 & 378.476178 & 376.6862 & 0.022809 \\
\hline$I 3-5$ & 379.525686 & 380.7002 & 0.014769 & 383.043983 & 380.8832 & 0.026020 \\
\hline$I 3-6$ & 375.225896 & 376.0348 & 0.010753 & 378.683492 & 376.2053 & 0.031496 \\
\hline
\end{tabular}


Table C.1.2: Output Temperatures for Detailed and Sub-models 5 and 6 and Corresponding Errors of GaN Model with Four-Port Configuration

\begin{tabular}{|c|c|c|c|c|c|c|}
\hline & \multicolumn{3}{|c|}{ Model-5 } & \multicolumn{3}{c|}{ Model-6 } \\
\hline Output & T_full & T_sub & Error & T full & T_sub & Error \\
\hline & $\mathbf{( K )}$ & $\mathbf{( K )}$ & & $(\mathbf{K})$ & $(\mathbf{K})$ & \\
\hline$I-\max$ & 401.424425 & 403.3787 & 0.019268 & 401.110493 & 403.0060 & 0.018747 \\
\hline$I 1-1$ & 376.279220 & 374.3940 & 0.024715 & 371.949263 & 374.1022 & 0.029923 \\
\hline$I 1-2$ & 380.442193 & 381.5630 & 0.013933 & 376.115668 & 381.2574 & 0.067552 \\
\hline$I I-3$ & 376.687603 & 375.5914 & 0.014294 & 372.542489 & 375.2812 & 0.037753 \\
\hline$I I-4$ & 380.343760 & 377.9898 & 0.029299 & 380.071092 & 377.6802 & 0.029860 \\
\hline$I I-5$ & 385.948666 & 386.6942 & 0.008674 & 385.672732 & 386.3695 & 0.008133 \\
\hline$I I-6$ & 380.921802 & 379.3579 & 0.019326 & 380.646190 & 379.0386 & 0.019934 \\
\hline$I 2-1$ & 377.976008 & 378.8720 & 0.011491 & 377.699037 & 378.5631 & 0.011121 \\
\hline$I 2-2$ & 384.314442 & 388.5814 & 0.050608 & 384.033349 & 388.2450 & 0.050119 \\
\hline$I 2-3$ & 378.834872 & 380.0660 & 0.015617 & 378.554440 & 379.7632 & 0.015388 \\
\hline$I 2-4$ & 382.208322 & 379.8611 & 0.028552 & 381.920442 & 379.5563 & 0.028859 \\
\hline$I 2-5$ & 388.470114 & 386.5764 & 0.021405 & 388.177785 & 386.2585 & 0.021766 \\
\hline$I 2-6$ & 382.880011 & 380.1330 & 0.033144 & 382.588960 & 379.8259 & 0.033456 \\
\hline$I 3-1$ & 377.637773 & 378.6715 & 0.013315 & 381.110204 & 378.3686 & 0.033801 \\
\hline$I 3-2$ & 383.548889 & 386.9515 & 0.040726 & 386.850874 & 386.6460 & 0.002359 \\
\hline$I 3-3$ & 378.509079 & 380.2845 & 0.022614 & 381.712504 & 379.9621 & 0.021421 \\
\hline$I 3-4$ & 378.444098 & 376.6634 & 0.022700 & 374.581065 & 376.3708 & 0.023997 \\
\hline$I 3-5$ & 383.012522 & 380.8597 & 0.025934 & 379.358863 & 380.5552 & 0.015075 \\
\hline$I 3-6$ & 378.655553 & 376.1833 & 0.031431 & 375.057750 & 375.8926 & 0.011123 \\
\hline
\end{tabular}

Table C.1.3: Output Temperatures for Detailed and Sub-models 7 and 8 and Corresponding Errors of GaN Model with Four-Port Configuration

\begin{tabular}{|c|c|c|c|c|c|c|}
\hline & \multicolumn{3}{|c|}{ Model-7 } & \multicolumn{3}{c|}{ Model-8 } \\
\hline Output & T_full & T_sub & Error & T_full & T_sub & Error \\
\hline & $\mathbf{( K )}$ & $\mathbf{( K )}$ & & $\mathbf{( K )}$ & $\mathbf{( K )}$ & \\
\hline$I-\max$ & 400.129321 & 401.8566 & 0.017250 & 396.425717 & 397.8427 & 0.014695 \\
\hline$I I-I$ & 371.101498 & 373.1874 & 0.029337 & 372.007359 & 369.8648 & 0.029755 \\
\hline$I 1-2$ & 375.268368 & 380.3048 & 0.066913 & 376.161352 & 376.8739 & 0.009356 \\
\hline$I I-3$ & 371.707607 & 374.3179 & 0.036402 & 372.452728 & 370.8623 & 0.021951 \\
\hline$I 1-4$ & 379.186365 & 376.7129 & 0.031236 & 375.925792 & 373.2340 & 0.035453 \\
\hline$I 1-5$ & 384.784434 & 385.3565 & 0.006747 & 381.498365 & 381.7198 & 0.002717 \\
\hline$I 1-6$ & 379.775579 & 378.0503 & 0.021627 & 376.539730 & 374.5039 & 0.026598 \\
\hline$I 2-I$ & 380.676892 & 377.5983 & 0.038160 & 373.481830 & 374.1289 & 0.008806 \\
\hline$I 2-2$ & 386.895966 & 387.2009 & 0.003509 & 379.772373 & 383.4707 & 0.046361 \\
\hline$I 2-3$ & 381.269490 & 378.8271 & 0.030053 & 374.371938 & 375.4430 & 0.014401 \\
\hline$I 2-4$ & 377.155963 & 378.6031 & 0.018756 & 373.730154 & 375.1592 & 0.019382 \\
\hline$I 2-5$ & 383.546467 & 385.2686 & 0.020613 & 380.075396 & 381.7139 & 0.020462 \\
\hline$I 2-6$ & 378.107177 & 378.8744 & 0.009823 & 374.699509 & 375.4492 & 0.010036 \\
\hline
\end{tabular}




\begin{tabular}{|l|l|l|l|l|l|l|}
\hline$I 3-1$ & 380.161606 & 377.4223 & 0.034172 & 376.597098 & 374.0046 & 0.033846 \\
\hline$I 3-2$ & 385.894675 & 385.6938 & 0.002339 & 382.283089 & 382.2489 & 0.000416 \\
\hline$I 3-3$ & 380.777429 & 378.9637 & 0.022453 & 377.232704 & 375.3964 & 0.023776 \\
\hline$I 3-4$ & 373.635369 & 375.4558 & 0.024722 & 373.577209 & 372.1417 & 0.019510 \\
\hline$I 3-5$ & 378.406397 & 379.6023 & 0.015253 & 378.093188 & 376.1629 & 0.024718 \\
\hline$I 3-6$ & 374.124718 & 374.9872 & 0.011636 & 373.821361 & 371.6971 & 0.028776 \\
\hline
\end{tabular}

Table C.1.4: Output Temperatures for Detailed and Sub-models 3 and 4 and Corresponding Errors of GaN Model with Five-Port Configuration

\begin{tabular}{|c|c|c|c|c|c|c|}
\hline & \multicolumn{3}{|c|}{ Model-3 } & \multicolumn{3}{c|}{ Model-4 } \\
\hline Output & T_full & T_sub & Error & T full & T_sub & Error \\
\hline & $\mathbf{( K )}$ & $\mathbf{( K )}$ & & $(\mathbf{K})$ & $\mathbf{( K )}$ & \\
\hline$I-m a x$ & 401.270520 & 405.6071 & 0.042822 & 401.444803 & 405.8197 & 0.043126 \\
\hline$I 1-1$ & 372.042118 & 375.1492 & 0.043129 & 376.295085 & 375.3859 & 0.011917 \\
\hline$I 1-2$ & 376.208840 & 382.2970 & 0.079888 & 380.456404 & 382.5333 & 0.025814 \\
\hline$I 1-3$ & 372.640068 & 377.1429 & 0.061988 & 376.698848 & 377.4043 & 0.009198 \\
\hline$I 1-4$ & 376.247013 & 379.9431 & 0.048475 & 376.427219 & 380.1939 & 0.049285 \\
\hline$I 1-5$ & 381.922821 & 387.9782 & 0.073916 & 382.103078 & 388.2329 & 0.07466 \\
\hline$I 1-6$ & 377.075942 & 381.0069 & 0.051001 & 377.248923 & 381.2554 & 0.051865 \\
\hline$I 2-1$ & 381.709384 & 380.9528 & 0.009259 & 381.886776 & 381.2116 & 0.008245 \\
\hline$I 2-2$ & 387.937108 & 389.6147 & 0.019077 & 388.114267 & 389.8591 & 0.019802 \\
\hline$I 2-3$ & 382.292999 & 382.4265 & 0.001622 & 382.462221 & 382.6774 & 0.002609 \\
\hline$I 2-4$ & 382.061111 & 381.6296 & 0.005258 & 382.233366 & 381.8748 & 0.004360 \\
\hline$I 2-5$ & 388.322290 & 388.0070 & 0.003570 & 388.494058 & 388.2419 & 0.002849 \\
\hline$I 2-6$ & 382.735802 & 381.2658 & 0.017767 & 382.900077 & 381.5021 & 0.016863 \\
\hline$I 3-1$ & 381.264865 & 380.7821 & 0.005941 & 377.666157 & 381.0306 & 0.043319 \\
\hline$I 3-2$ & 387.010081 & 388.3763 & 0.015702 & 383.576467 & 388.6067 & 0.060187 \\
\hline$I 3-3$ & 381.873262 & 381.8238 & 0.000604 & 378.533109 & 382.0574 & 0.044876 \\
\hline$I 3-4$ & 374.743101 & 378.9757 & 0.056629 & 378.476178 & 379.2118 & 0.009374 \\
\hline$I 3-5$ & 379.525686 & 382.8621 & 0.041954 & 383.043983 & 383.0909 & 0.000565 \\
\hline$I 3-6$ & 375.225896 & 378.7734 & 0.047158 & 378.683492 & 379.0105 & 0.004156 \\
\hline
\end{tabular}
Table C.1.5: Output Temperatures for Detailed and Sub-models 5 and 6 and
Corresponding Errors of GaN Model with Five-Port Configuration

\begin{tabular}{|c|c|c|c|c|c|c|}
\hline & \multicolumn{3}{|c|}{ Model-5 } & \multicolumn{3}{c|}{ Model-6 } \\
\hline Output & T_full & T_sub & Error & T_full & T_sub & Error \\
\hline & (K) & (K) & & (K) & (K) & \\
\hline$T-$ max & 401.424425 & 405.7919 & 0.043061 & 401.110493 & 405.4359 & 0.042779 \\
\hline$I I-1$ & 376.279220 & 375.3606 & 0.012043 & 371.949263 & 374.9920 & 0.042290 \\
\hline$I 1-2$ & 380.442193 & 382.5080 & 0.025681 & 376.115668 & 382.1371 & 0.079109 \\
\hline$I I-3$ & 376.687603 & 377.3777 & 0.008999 & 372.542489 & 376.9657 & 0.060974 \\
\hline
\end{tabular}




\begin{tabular}{|l|l|l|l|l|l|l|}
\hline$I I-4$ & 380.343760 & 380.1660 & 0.002212 & 380.071092 & 379.7692 & 0.003770 \\
\hline$I I-5$ & 385.948666 & 388.2053 & 0.026256 & 385.672732 & 387.8018 & 0.024851 \\
\hline$I 1-6$ & 380.921802 & 381.2289 & 0.003795 & 380.646190 & 380.8294 & 0.002272 \\
\hline$I 2-1$ & 377.976008 & 381.1800 & 0.041089 & 377.699037 & 380.7605 & 0.039402 \\
\hline$I 2-2$ & 384.314442 & 389.8290 & 0.065405 & 384.033349 & 389.4290 & 0.064208 \\
\hline$I 2-3$ & 378.834872 & 382.6479 & 0.048367 & 378.554440 & 382.2332 & 0.046831 \\
\hline$I 2-4$ & 382.208322 & 381.8409 & 0.004469 & 381.920442 & 381.4275 & 0.006017 \\
\hline$I 2-5$ & 388.470114 & 388.2095 & 0.002946 & 388.177785 & 387.8108 & 0.004162 \\
\hline$I 2-6$ & 382.880011 & 381.4712 & 0.016998 & 382.588960 & 381.0697 & 0.018395 \\
\hline$I 3-1$ & 377.637773 & 380.9933 & 0.043220 & 381.110204 & 380.5686 & 0.006677 \\
\hline$I 3-2$ & 383.548889 & 388.5713 & 0.060113 & 386.850874 & 388.1677 & 0.015162 \\
\hline$I 3-3$ & 378.509079 & 382.0235 & 0.044765 & 381.712504 & 381.6144 & 0.001201 \\
\hline$I 3-4$ & 378.444098 & 379.1743 & 0.009309 & 374.581065 & 378.7628 & 0.056070 \\
\hline$I 3-5$ & 383.012522 & 383.0546 & 0.000507 & 379.358863 & 382.6510 & 0.041484 \\
\hline$I 3-6$ & 378.655553 & 378.9743 & 0.004052 & 375.057750 & 378.5549 & 0.046593 \\
\hline
\end{tabular}

Table C.1.6: Output Temperatures for Detailed and Sub-models 7 and 8 and
Corresponding Errors of GaN Model with Five-Port Configuration

\begin{tabular}{|c|c|c|c|c|c|c|}
\hline & \multicolumn{3}{|c|}{ Model-7 } & \multicolumn{3}{c|}{ Model-8 } \\
\hline Output & T_full & T_sub & Error & T full & T_sub & Error \\
\hline & $(\mathbf{K})$ & $\mathbf{( K )}$ & & (K) & (K) & \\
\hline$I-$ max & 400.129321 & 404.3345 & 0.041997 & 396.425717 & 400.4421 & 0.041653 \\
\hline$I 1-1$ & 371.101498 & 373.8512 & 0.038673 & 372.007359 & 369.9323 & 0.028817 \\
\hline$I 1-2$ & 375.268368 & 380.9912 & 0.076032 & 376.161352 & 377.0456 & 0.011610 \\
\hline$I 1-3$ & 371.707607 & 375.7006 & 0.055684 & 372.452728 & 371.3765 & 0.014854 \\
\hline$I 1-4$ & 379.186365 & 378.5425 & 0.008131 & 375.925792 & 374.3166 & 0.021194 \\
\hline$I 1-5$ & 384.784434 & 386.5575 & 0.020913 & 381.498365 & 382.2701 & 0.009469 \\
\hline$I 1-6$ & 379.775579 & 379.6040 & 0.002151 & 376.539730 & 375.3861 & 0.015072 \\
\hline$I 2-1$ & 380.676892 & 379.4674 & 0.014992 & 373.481830 & 375.0304 & 0.021074 \\
\hline$I 2-2$ & 386.895966 & 388.1947 & 0.014946 & 379.772373 & 383.9076 & 0.051838 \\
\hline$I 2-3$ & 381.269490 & 380.9628 & 0.003774 & 374.371938 & 376.5676 & 0.029523 \\
\hline$I 2-4$ & 377.155963 & 380.1547 & 0.038866 & 373.730154 & 375.7563 & 0.027481 \\
\hline$I 2-5$ & 383.546467 & 386.5827 & 0.036342 & 380.075396 & 382.2962 & 0.027734 \\
\hline$I 2-6$ & 378.107177 & 379.8390 & 0.022172 & 374.699509 & 375.5546 & 0.011447 \\
\hline$I 3-1$ & 380.161606 & 379.2581 & 0.011271 & 376.597098 & 374.7187 & 0.024523 \\
\hline$I 3-2$ & 385.894675 & 386.9254 & 0.012000 & 382.283089 & 382.5741 & 0.003537 \\
\hline$I 3-3$ & 380.777429 & 380.3609 & 0.005157 & 377.232704 & 375.9729 & 0.016312 \\
\hline$I 3-4$ & 373.635369 & 377.4921 & 0.052376 & 373.577209 & 373.0570 & 0.007070 \\
\hline$I 3-5$ & 378.406397 & 381.4083 & 0.038286 & 378.093188 & 377.0543 & 0.013303 \\
\hline$I 3-6$ & 374.124718 & 377.2697 & 0.042428 & 373.821361 & 372.7762 & 0.014158 \\
\hline
\end{tabular}




\section{C.2 GaAs Based Power Amplifier}

Table C.2.1: Output Temperatures for Detailed and Sub-models 3 and 4 and Corresponding Errors of GaAs Model

\begin{tabular}{|c|c|c|c|c|c|c|}
\hline & \multicolumn{3}{|c|}{ Model-3 } & \multicolumn{3}{c|}{ Model-4 } \\
\hline Output & Tfull & T sub & Error & T full & T sub & Error \\
\hline & (K) & (K) & & $(\mathbf{K})$ & (K) & \\
\hline$T-\max$ & 466.959273 & 467.4175 & 0.002745 & 462.113724 & 463.0620 & 0.005849 \\
\hline$I I-1$ & 400.933000 & 398.8891 & 0.020250 & 397.900300 & 395.9656 & 0.019762 \\
\hline$I 1-2$ & 465.186000 & 464.0476 & 0.006892 & 462.806500 & 460.3526 & 0.015072 \\
\hline$I I-3$ & 454.038100 & 451.5427 & 0.016200 & 450.005000 & 448.0243 & 0.013204 \\
\hline$I 2-1$ & 402.905900 & 401.3975 & 0.014658 & 399.456900 & 398.4309 & 0.010316 \\
\hline$I 2-2$ & 469.005700 & 466.8773 & 0.012594 & 464.174500 & 463.3053 & 0.005294 \\
\hline$I 2-3$ & 455.570600 & 454.2839 & 0.008271 & 451.743900 & 450.7203 & 0.006746 \\
\hline$I 3-1$ & 402.657000 & 401.7431 & 0.008902 & 400.432000 & 398.8297 & 0.015954 \\
\hline$I 3-2$ & 468.480400 & 467.9114 & 0.003377 & 466.571200 & 464.3899 & 0.013095 \\
\hline$I 3-3$ & 455.620700 & 455.0404 & 0.003729 & 452.422700 & 451.4948 & 0.006088 \\
\hline$I 4-1$ & 400.628900 & 398.5822 & 0.020339 & 398.183400 & 395.6680 & 0.025619 \\
\hline$I 4-2$ & 463.212400 & 462.3041 & 0.005565 & 460.428400 & 458.7241 & 0.010623 \\
\hline$I 4-3$ & 452.572000 & 450.9469 & 0.010651 & 448.677000 & 447.5657 & 0.007475 \\
\hline
\end{tabular}

Table C.2.2: Output Temperatures for Detailed and Sub-models 5 and 6 and Corresponding Errors of GaAs Model

\begin{tabular}{|c|c|c|c|c|c|c|}
\hline & \multicolumn{3}{|c|}{ Model-5 } & \multicolumn{3}{c|}{ Model-6 } \\
\hline Output & T_full & T_sub & Error & T_full & T_sub & Error \\
\hline & $\mathbf{( K )}$ & $\mathbf{( K )}$ & & $(\mathbf{K})$ & $(\mathbf{K})$ & \\
\hline$I-$ max & 451.281164 & 454.2994 & 0.019951 & 455.506945 & 457.4616 & 0.012570 \\
\hline$I 1-1$ & 390.150000 & 389.6311 & 0.005756 & 392.735500 & 392.0576 & 0.007310 \\
\hline$I 1-2$ & 455.395800 & 452.4566 & 0.018914 & 457.169700 & 455.4303 & 0.011067 \\
\hline$I 1-3$ & 441.449000 & 440.6346 & 0.005758 & 445.662800 & 443.3817 & 0.015660 \\
\hline$I 2-1$ & 392.685400 & 391.9284 & 0.008167 & 395.978000 & 394.3886 & 0.016560 \\
\hline$I 2-2$ & 458.118300 & 455.4911 & 0.016615 & 460.292400 & 458.3708 & 0.011988 \\
\hline$I 2-3$ & 445.479500 & 443.2676 & 0.015204 & 446.630100 & 446.0029 & 0.004277 \\
\hline$I 3-I$ & 392.786800 & 392.3772 & 0.004414 & 395.942000 & 394.8026 & 0.011876 \\
\hline$I 3-2$ & 456.599500 & 456.5642 & 0.000225 & 461.795000 & 459.4450 & 0.014525 \\
\hline$I 3-3$ & 446.606900 & 443.9252 & 0.018292 & 448.747800 & 446.7052 & 0.013732 \\
\hline$I 4-I$ & 389.684800 & 389.0874 & 0.006661 & 392.960600 & 391.5773 & 0.014880 \\
\hline$I 4-2$ & 452.126200 & 451.2300 & 0.005891 & 455.688800 & 453.9853 & 0.010942 \\
\hline$I 4-3$ & 442.191300 & 440.2070 & 0.013955 & 445.340500 & 442.9578 & 0.016394 \\
\hline
\end{tabular}


Table C.2.3: Output Temperatures for Detailed and Sub-models 7 and 8 and Corresponding Errors of GaAs Model

\begin{tabular}{|c|c|c|c|c|c|c|}
\hline & \multicolumn{3}{|c|}{ Model-7 } & \multicolumn{3}{c|}{ Model-8 } \\
\hline Output & T_full & T_sub & Error & T_full & T_sub & Error \\
\hline & (K) & (K) & & (K) & (K) & \\
\hline$I-$ max & 464.222617 & 464.9249 & 0.004276 & 467.302146 & 467.8300 & 0.003155 \\
\hline$I I-1$ & 400.224800 & 397.2545 & 0.029636 & 401.043500 & 399.1539 & 0.018701 \\
\hline$I 1-2$ & 464.322900 & 461.9563 & 0.014402 & 465.478300 & 464.3668 & 0.006717 \\
\hline$I I-3$ & 450.852300 & 449.5363 & 0.008724 & 453.572300 & 451.8469 & 0.011235 \\
\hline$I 2-1$ & 401.221300 & 399.7264 & 0.014769 & 403.023800 & 401.6695 & 0.013146 \\
\hline$I 2-2$ & 465.458400 & 464.8165 & 0.003880 & 467.299200 & 467.1675 & 0.000787 \\
\hline$I 2-3$ & 454.175800 & 452.2453 & 0.012521 & 454.684900 & 454.6033 & 0.000528 \\
\hline$I 3-1$ & 401.052500 & 400.0924 & 0.009501 & 402.939200 & 402.0012 & 0.009112 \\
\hline$I 3-2$ & 468.760400 & 465.8801 & 0.017067 & 468.241600 & 468.2030 & 0.000229 \\
\hline$I 3-3$ & 455.189500 & 453.0096 & 0.014047 & 456.513500 & 455.3616 & 0.007360 \\
\hline$I 4-1$ & 398.216100 & 396.9803 & 0.012582 & 400.954200 & 398.9048 & 0.020300 \\
\hline$I 4-2$ & 462.539400 & 460.3057 & 0.013743 & 462.988000 & 462.7095 & 0.001709 \\
\hline$I 4-3$ & 449.868800 & 449.0649 & 0.005364 & 451.422300 & 451.3163 & 0.000700 \\
\hline
\end{tabular}

Table C.2.4: Output Temperatures for Detailed and Sub-models 9 and 10 and Corresponding Errors of GaAs Model

\begin{tabular}{|c|c|c|c|c|c|c|}
\hline & \multicolumn{3}{|c|}{ Model-9 } & \multicolumn{3}{c|}{ Model-10 } \\
\hline Output & T_full & T_sub & Error & T_full & T_sub & Error \\
\hline & $\mathbf{( K )}$ & $\mathbf{( K )}$ & & $(\mathbf{K})$ & $(\mathbf{K})$ & \\
\hline$I-\max$ & 465.084253 & 465.8267 & 0.004497 & 457.366776 & 459.2074 & 0.011696 \\
\hline$I I-1$ & 399.980800 & 397.8279 & 0.021533 & 395.990700 & 393.2655 & 0.028390 \\
\hline$I I-2$ & 464.760300 & 462.6823 & 0.012612 & 457.636300 & 456.9407 & 0.004413 \\
\hline$I I-3$ & 450.488000 & 450.2358 & 0.001676 & 445.520400 & 444.8024 & 0.004934 \\
\hline$I 2-1$ & 401.694700 & 400.3316 & 0.013404 & 395.823800 & 395.6746 & 0.001557 \\
\hline$I 2-2$ & 466.878300 & 465.5528 & 0.007943 & 460.161500 & 459.9264 & 0.001468 \\
\hline$I 2-3$ & 454.038000 & 452.9782 & 0.006880 & 449.408100 & 447.4856 & 0.012867 \\
\hline$I 3-1$ & 401.155500 & 400.6947 & 0.004555 & 396.669900 & 396.0972 & 0.005924 \\
\hline$I 3-2$ & 468.646500 & 466.6196 & 0.012019 & 463.564200 & 461.0326 & 0.015478 \\
\hline$I 3-3$ & 455.850500 & 453.7529 & 0.013459 & 448.752200 & 448.2305 & 0.003507 \\
\hline$I 4-1$ & 399.785000 & 397.6024 & 0.021873 & 393.453200 & 392.9408 & 0.005483 \\
\hline$I 4-2$ & 462.514700 & 461.0795 & 0.008831 & 458.526200 & 455.5433 & 0.018816 \\
\hline$I 4-3$ & 451.453500 & 449.7890 & 0.010990 & 445.795100 & 444.4757 & 0.009050 \\
\hline
\end{tabular}




\section{C.3 Package Model}

Table C.3.1: Output Temperatures for Detailed and Sub-models 3 and 4 and Corresponding Errors of Four-Package Model with Right-side BC

\begin{tabular}{|c|c|c|c|c|c|c|}
\hline & \multicolumn{3}{|c|}{ Model -3 } & \multicolumn{3}{c|}{ Model -4 } \\
\hline Output & T_full & T sub & Error & T full & T_sub & Error \\
\hline & $\mathbf{( K )}$ & $\mathbf{( K )}$ & & $(\mathbf{K})$ & $(\mathbf{K})$ & \\
\hline$T-$ max & 348.988662 & 344.8530 & 0.084421 & 349.009553 & 344.9260 & 0.083322 \\
\hline$P 1$ & 331.921227 & 329.4067 & 0.078773 & 331.856623 & 329.4482 & 0.075602 \\
\hline$P 2$ & 334.648289 & 331.8775 & 0.079969 & 334.636274 & 331.9240 & 0.078307 \\
\hline$P 3$ & 334.619682 & 331.8755 & 0.079267 & 334.666009 & 331.9220 & 0.079156 \\
\hline$P 4$ & 331.840866 & 329.4088 & 0.076382 & 331.939772 & 329.4503 & 0.077943 \\
\hline$P 5$ & 334.550005 & 331.8734 & 0.077470 & 334.486828 & 331.9199 & 0.074432 \\
\hline$P 6$ & 340.890717 & 338.7699 & 0.051865 & 340.879809 & 338.8233 & 0.050306 \\
\hline$P 7$ & 340.861495 & 338.7585 & 0.051466 & 340.910327 & 338.8119 & 0.051293 \\
\hline$P 8$ & 334.469492 & 331.8656 & 0.075542 & 334.570353 & 331.9121 & 0.076894 \\
\hline$P 9$ & 334.349572 & 331.8754 & 0.072029 & 334.287852 & 331.9219 & 0.069003 \\
\hline$P 10$ & 340.685547 & 338.7701 & 0.047079 & 340.676126 & 338.8235 & 0.045546 \\
\hline$P 11$ & 340.656272 & 338.7725 & 0.046334 & 340.706648 & 338.8260 & 0.046200 \\
\hline$P 12$ & 334.268906 & 331.8700 & 0.070002 & 334.371350 & 331.9165 & 0.071421 \\
\hline$P 13$ & 331.357038 & 329.4121 & 0.062026 & 331.296592 & 329.4536 & 0.058888 \\
\hline$P 14$ & 334.084109 & 331.8789 & 0.064699 & 334.076225 & 331.9254 & 0.063118 \\
\hline$P 15$ & 334.055377 & 331.8771 & 0.063963 & 334.105960 & 331.9237 & 0.063985 \\
\hline$P 16$ & 331.276272 & 329.4124 & 0.059594 & 331.379627 & 329.4539 & 0.061369 \\
\hline
\end{tabular}

Table C.3.2: Output Temperatures for Detailed and Sub-models 3 and 4 and Corresponding Errors of Four-Package Model with Back-side BC

\begin{tabular}{|c|c|c|c|c|c|c|}
\hline & \multicolumn{3}{|c|}{ Model -3 } & \multicolumn{3}{c|}{ Model -4 } \\
\hline Output & T full & T sub & Error & T full & T sub & Error \\
\hline & (K) & (K) & & (K) & (K) & \\
\hline$T-$ max & 345.645632 & 344.2785 & 0.029951 & 345.745448 & 344.3766 & 0.029923 \\
\hline$P 1$ & 322.235017 & 320.0867 & 0.096619 & 322.311589 & 320.1627 & 0.096313 \\
\hline$P 2$ & 326.040444 & 323.5012 & 0.097512 & 326.127268 & 323.5846 & 0.097319 \\
\hline$P 3$ & 326.035241 & 323.4982 & 0.097446 & 326.134065 & 323.5817 & 0.097664 \\
\hline$P 4$ & 322.220174 & 320.0896 & 0.095885 & 322.329555 & 320.1654 & 0.096919 \\
\hline$P 5$ & 326.115202 & 323.4945 & 0.100352 & 326.191942 & 323.5780 & 0.099799 \\
\hline$P 6$ & 335.001376 & 333.1749 & 0.052183 & 335.089624 & 333.2696 & 0.051868 \\
\hline$P 7$ & 334.996058 & 333.1592 & 0.052488 & 335.096905 & 333.2546 & 0.052492 \\
\hline$P 8$ & 326.100285 & 323.4842 & 0.100232 & 326.210617 & 323.5682 & 0.100815 \\
\hline
\end{tabular}




\begin{tabular}{|c|c|c|c|c|c|c|}
\hline$P 9$ & 326.109430 & 323.4973 & 0.100045 & 326.185550 & 323.5806 & 0.099480 \\
\hline$P 10$ & 334.995292 & 333.1755 & 0.052001 & 335.083052 & 333.2703 & 0.051670 \\
\hline$P 11$ & 334.989898 & 333.1786 & 0.051766 & 335.090370 & 333.2731 & 0.051788 \\
\hline$P 12$ & 326.094303 & 323.4892 & 0.099834 & 326.204312 & 323.5727 & 0.100427 \\
\hline$P 13$ & 322.218686 & 320.0941 & 0.095622 & 322.293578 & 320.1699 & 0.095260 \\
\hline$P 14$ & 326.023699 & 323.5027 & 0.096873 & 326.109230 & 323.5857 & 0.096653 \\
\hline$P 15$ & 326.018311 & 323.5003 & 0.096778 & 326.116103 & 323.5837 & 0.096967 \\
\hline$P 16$ & 322.203287 & 320.0944 & 0.094981 & 322.311760 & 320.1700 & 0.095992 \\
\hline
\end{tabular}




\section{References}

[1] N. K. Verghese, S. S. Lee, D. J. Allstot "A Unified Approach to Simulating Electrical and Thermal Substrate Coupling Interactions in ICs" IEEE/ACM International Conference on Computer-Aided Design, ICCAD-93., Digest of Technical Papers, Pages: 422 - 426, 7 - 11 November, 1993

[2] E. Savrun "Packaging Considerations for Very High Temperature Microsystems" IEEE Proceedings of Sensors, Volume: 2, Pages: 1139 - 1143, 12 - 14 June, 2002

[3] Y. Joshi, A. Baelmans, D. Copeland, C. J. M. Lasance, J. Parry, J. Rantala "Challenges in Thermal Modeling of Electronics at the System Level: Summary of Panel Held at the Therminic 2000" IEEE Transactions on Components, Packaging and Manufacturing Technology, Part A, Volume: 24, Issue: 4, Pages: $611-613$, December, 2001

[4] D. Celo, X. Guo, P. K. Gunupudi, R. Khazaka, D. J. Walkey, T. Smy, M. S. Nakhla "Hierarchical Thermal Analysis of Large IC Modules" IEEE Transactions on Components and Packaging Technologies, Volume: 28, No: 2, Pages: 207 217, June, 2005

[5] S. Shidore, A. Sahrapour "Delphi Compact Models Revolutionize Thermal Design" in www.coolingzone.com, Pages: 1-6, July, 2001

[6] T. Smy, D. Walkey, S. K. Dew "A 3D Thermal Simulation Tool for Integrated Devices - Atar" IEEE Transactions on Computer-Aided Design of Integrated Circuits and Systems, Volume: 20, No: 1, Pages: 1-11, January, 2001

[7] T. Smy, D. Walkey, S. K. Dew "Transient 3D Heat Flow Analysis for Integrated Circuit Devices Using the Transmission Line Matrix Method on a Quad Tree Mesh "Solid State Electronics, Volume: 45, No: 7, Pages: 1137 - 1148, July, 2001

[8] T. Smy, D. Walkey, M. Shams, R. V. Joshi "Simulation of Local Heating in VLSI Backend Structures Using Atar" Advanced Metallization Conference 2001, Page: 
355, October, 2001

[9] V. V. Calmidi, R. L. Mahajan, "Optimization for Thermal and Electrical Performance for a Flip-chip Package Using Physical-neural Network Modeling” 47th Electronic Components and Technology Conference, Pages: 1163 - 1169, 18 - 21 May, 1997

[10] J. W. Dally "Packaging of Electronic System - A Mechanical Engineering Approach" McGraw-Hill Publishing Company, 1990

[11] D. P. Seraphim, R. Lasky, C. Y. Li "Principles of Electronic Packaging" McGraw-Hill Book Company, 1989

[12] J. H. Lau "Electronic Packaging : Design, Materials, Process, and Reliability" McGraw-Hill, 1998

[13] C. A. Harper "Electronic Packaging \& Interconnection Handbook" Second Edition, McGraw-Hill, 1997

[14] G. R. Blackwell "The Electronic Packaging Handbook" IEEE Press, 2000

[15] H. Carslaw, J. L. Jaeger "Conduction of Heat in Solids" Second Edition, Oxford University Press, London, 1959

[16] "NeuroModeler Version 1.5", Prof. Q.J. Zhang, Department of Electronics, Carleton University, Ottawa, Canada K1S 5B6. www.doe.carleton.ca/ qjz

[17] Q. J. Zhang, K. C. Gupta “Neural Networks for RF and Microwave Design” Artech House, 2000

[18] S. Haykin "Neural Networks - A Comprehensive Foundation" Second Edition, Prentice Hall, 1999

[19] Y. H. Fang, M. C. E. Yagoub, F. Wang, Q. J. Zhang “A New Macromodeling Approach for Nonlinear Microwave Circuits Based on Recurrent Neural Networks" IEEE Transactions on Microwave Theory and Techniques, Volume: 48, No: 12, Pages: 2335 - 2344, December, 2000

[20] M. Stinchcombe, H. White "Approximating and Learning Unknown Mappings Using Multilayer Feedforward Networks with Bounded Weights" International Joint Conference on Neural Networks, Volume: 3, Pages: 7 - 16, 17 - 21 June, 1990

[21] W. S. McCulloch, W. Pitts "A Logical Calculus of the Ideas Immanent in Nervous 
Activity" Bulletin of Mathematical Biophysics, Volume: 5, Pages: 115 - 133, 1990

[22] V. K. Devabhaktuni, Y. H. Fang, M. C. E. Yagoub, F. Wang, Q. J. Zhang "Neural Networks for Microwave Modeling: Model Development Issues and Nonlinear Modeling Techniques" International Journal of RF and Microwave CAE, Volume: 11, Pages: 4 - 21, 2001 (invited review)

[23] F. Rosenblatt "Principles of Neurodynamics; Perceptrons and the Theory of Brain Mechanisms" Spartan Books, Washington, 1962

[24] B. Muller, J. Reinhardt, M. T. Strickland "Neural Networks: An Introduction" Second Edition, Springer-Verlag Berlin Heidelberg, New York, 1995

[25] S. I. Gallant "Neural Network Learning and Expert Systems" Second Edition, 1994 AIChEJ, Volume: 40, No: 5, Pages: 826 - 838, 1994

[26] S. Tamura, M. Tateishi "Capabilities of a Four-Layered Feedforward Neural Network: Four Layers Versus Three" IEEE Transactions on Neural Networks, Volume: 8, Issue: 2, Pages: 251 - 255, March, 1997

[27] J. de Villiers, E. Barnard "Backpropagation Neural Nets with One and Two Hidden Layers" IEEE Transactions on Neural Networks, Volume: 4, Issue: 1, Pages: 136 - 141, January, 1993

[28] F. Wang, V. K. Devabhaktuni, C. Xi, Q. J. Zhang "Neural Networks Structures and Training Algorithms for RF and Microwave Applications" International Journal of RF and Microwave CAE, Special Issue on Applications of Artificial Neural Networks to RF and Microwave Design, Volume: 9, Pages: 216 - 240, 1999

[29] D. E. Rumelhart, G. E. Hinton, R. J. Williams "Learning Internal Representations by Error Propagation" Parallel Distributed Processing, Volume: 1, The MIT Press, Cambridge, MA, 1986

[30] D. G. Luenberger "Linear and Nonlinear Programming" Second Edition, Reading, Mass.: Addison-Wesley, 1984

[31] W. H. Press, B. P. Flannery, S. A. Teukolsky, W. T. Vetterling "Numerical Recipes in C: The Art of Scientific Computing" Second Edition, Cambridge University Press, 1992

[32] R. Fletcher, C. M. Reeves "Function Minimization by Conjugate Gradients" The 
Computer Journal, Volume: 6, Pages: 149 - 154, 1964

[33] E. Polak, G. Ribière "Note sur la Convergence de Directions Conjugées" Revue Francaise Informat Recherche Operationelle, Volume: 16, Pages: 35 - 43, 1969

[34] X. Guo "Efficient Thermal Simulation Using Model Reduction Techniques" M.A.Sc Thesis, 2004 